\title{
Accelerator Studies of Neutrino Oscillations
}

\author{
A. Ereditato ${ }^{1}$ and P. Migliozzi ${ }^{2}$ \\ 1) INFN, Sezione di Napoli, Italy \\ 2) CERN, Geneva, Switzerland and INFN, Sezione di Napoli, Italy
}

\begin{abstract}
The question of whether the neutrino has a non-vanishing mass plays a crucial role in particle physics. A massive neutrino would unambiguously reveal the existence of new physics beyond the Standard Model. In addition, it could have profound implications on astrophysics and cosmology, with effects on the evolution of the Universe. Experiments aiming at direct neutrino-mass measurements based on kinematics have not been able, so far, to measure the very small neutrino mass. Indirect measurements can be performed by exploiting reactions which may only occur for massive neutrinos. Neutrino oscillation is one of those processes. The mass difference between neutrino mass-eigenstates can be inferred from a phase measurement. This feature allows for high sensitivity experiments. Neutrinos from different sources can be used to search for oscillations: solar neutrinos, neutrinos produced in the interaction of cosmic rays with the atmosphere and artificially produced neutrinos from nuclear reactors and particle accelerators. The latter offer the possibility of choosing the relevant experimental features such as the flux flavour composition, the energy and the baseline distance from the source to the detector. This paper attempts to review the main accelerator experiments which have been performed and to outline the future projects. A brief introduction to the theory and phenomenology of neutrino oscillations is given to help in understanding the scope, the design and the performance of the different experiments.
\end{abstract}

(Submitted to La Rivista del Nuovo Cimento) 


\section{Contents}

1 Introduction $\quad 5$

2 Phenomenology of neutrino oscillations $r$

2.1 Neutrino oscillations in vacuum . . . . . . . . . . . . . . . 8

2.2 Quantum mechanics of neutrino oscillations . . . . . . . . . . . . . . . 11

2.3 Two-flavour oscillations . . . . . . . . . . . . . . . . . . . . 12

3 Detection of neutrino oscillations $\quad 13$

3.1 Disappearance experiments . . . . . . . . . . . . . . . . . . 15

3.1.1 Near/far test for disappearance experiments . . . . . . . . . . . . . . . . 15

3.1 .2 Energy measurement of $\nu_{\mu} \mathrm{CC}$ events $\ldots \ldots \ldots \ldots \ldots$

3.2 Appearance experiments . . . . . . . . . . . . . . . . . . . . . . 19

$3.2 .1 \quad \mathrm{NC} / \mathrm{CC}$ test for $\nu_{\mu} \leftrightarrow \nu_{\tau}$ oscillations . . . . . . . . . . . . . 20

3.2.2 Indirect appearance experiments searching for $\nu_{\mu} \leftrightarrow \nu_{e}$ oscillations . . . . . . 22

3.2.3 Direct appearance experiments searching for $\nu_{\mu} \leftrightarrow \nu_{\tau}$ oscillations . . . . . . . 23

3.2.4 Short baseline versus long baseline appearance experiments . . . . . . . . . . 24

4 Solar neutrinos $\quad 25$

4.1 Experimental results . . . . . . . . . . . . . . . . . . 25

4.2 The solar neutrino problem . . . . . . . . . . . . . . . . . . 31

5 Atmospheric neutrinos $\quad 31$

5.1 The atmospheric neutrino anomaly . . . . . . . . . . . . . 31

5.2 Zenith angle dependence and oscillation hypothesis . . . . . . . . . . . . 35

6 Neutrino beams from particle accelerators $\quad 37$

6.1 General features . . . . . . . . . . . . . . . . . . 37

6.2 The CERN WANF Wide Band Beam . . . . . . . . . . . . . . . . 39

6.3 The CERN-Gran Sasso CNGS beam . . . . . . . . . . . . . . . . . . . . 42

6.3.1 Optimisation for $\nu_{\mu} \leftrightarrow \nu_{\tau}$ oscillation searches . . . . . . . . . . . 44

6.3 .2 Beam line design . . . . . . . . . . . . . . . . . . 47

6.4 The Fermilab-Soudan NuMI beam . . . . . . . . . . . . . . . . . . 50

6.5 The KEK-Kamioka beam . . . . . . . . . . . . . . . . . . . . . . . 52

7 Neutrino oscillation experiments at CERN

7.1 Bubble chamber experiments: Gargamelle and BEBC . . . . . . . . . . . . . 55 


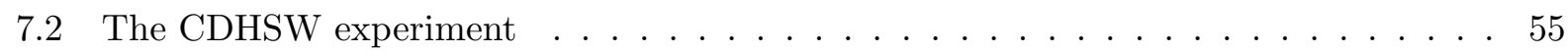

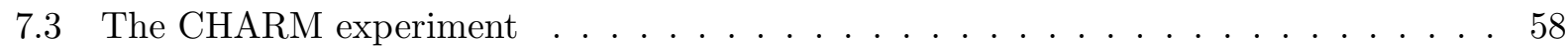

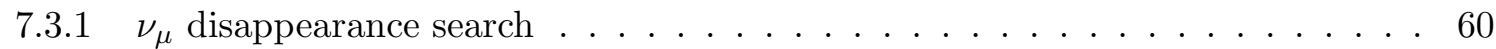

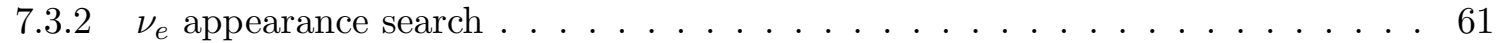

7.4 The CHARM II experiment . . . . . . . . . . . . . . . . . . . . 62

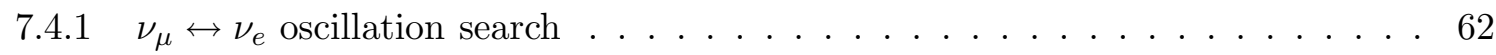

$7.4 .2 \nu_{\mu} \leftrightarrow \nu_{\tau}$ oscillation search . . . . . . . . . . . . . . 65

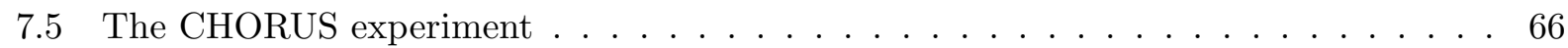

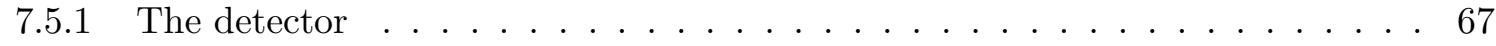

7.5 .2 Data analysis . . . . . . . . . . . . . . . . . 69

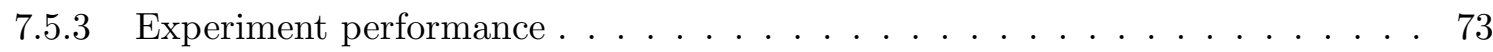

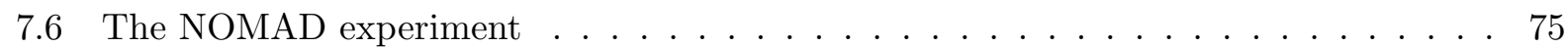

7.6 .1 Data analysis . . . . . . . . . . . . . . . . 76

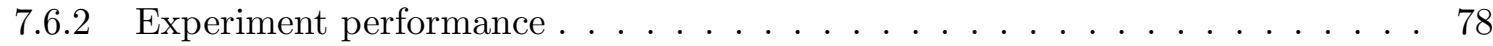

$8 \nu_{\mu} \leftrightarrow \nu_{e}$ oscillation searches at BNL $\quad \mathbf{8 0}$

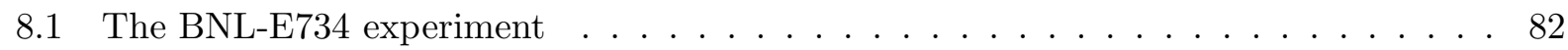

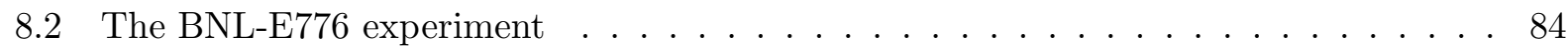

9 Neutrino oscillation searches at Fermilab $\quad 85$

9.1 The CCFR experiment . . . . . . . . . . . . . . . . 88

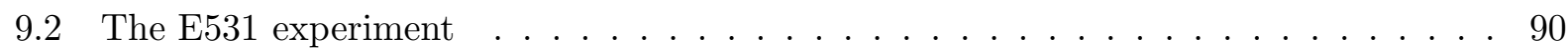

10 Search for neutrino oscillations with low energy accelerators $\quad 91$

10.1 The LSND experiment . . . . . . . . . . . . . . . . . . . . . . . 91

10.1 .1 The detector . . . . . . . . . . . . . . . . . 94

10.1.2 $\bar{\nu}_{\mu} \leftrightarrow \bar{\nu}_{e}$ oscillation search from muonic decay at rest . . . . . . . . . . . 95

10.1.3 $\nu_{\mu} \leftrightarrow \nu_{e}$ oscillation search from pion decay in flight . . . . . . . . . . . . . 97

10.2 The KARMEN experiment ～. . . . . . . . . . . . . . . . . . . . . . . . . 99

10.2.1 Oscillation search with the KARMEN1 detector . . . . . . . . . . . . 102

10.2.2 Oscillation search with the KARMEN2 detector . . . . . . . . . . . . 104

11 Scenarios for neutrino oscillations $\quad 105$

11.1 Three-flavour versus sterile neutrino scheme . . . . . . . . . . . . . 105

12 Proposed short baseline experiments $\quad 108$

12.1 Experiments for $\nu_{\mu} \leftrightarrow \nu_{\tau}$ oscillation searches . . . . . . . . . . . . 108 
12.2 The I216 experiment in the CERN-PS neutrino beam . . . . . . . . . . . 111

12.3 BooNE at the Fermilab Booster . . . . . . . . . . . . . . . . . . . 111

13 Long baseline experiments $\quad 116$

13.1 The K2K experiment in the KEK-Kamioka beam . . . . . . . . . . . . . 116

13.2 The MINOS experiment in the NuMi beam . . . . . . . . . . . . . . 125

13.3 The ICANOE experiment in the CNGS beam . . . . . . . . . . . . . . . 128

13.3 .1 The ICARUS detector . . . . . . . . . . . . . . . . . . . 129

13.3.2 The NOE calorimeter . . . . . . . . . . . . . . . . 134

13.3 .3 ICANOE performance . . . . . . . . . . . . . . . 134

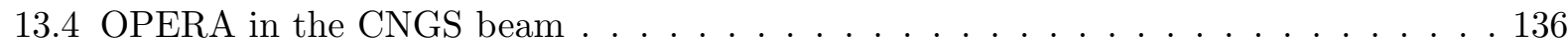

13.4 .1 Detector strucure . . . . . . . . . . . . . . . . . . 139

13.4.2 Detection efficiency and background . . . . . . . . . . . . . 140

13.4.3 Sensitivity to $\nu_{\mu} \leftrightarrow \nu_{\tau}$ oscillations . . . . . . . . . . . . . 141

14 Future experiments with beams from muon storage rings $\quad 142$

14.1 Neutrino beams from muon decay . . . . . . . . . . . . . . . . . 142

14.2 LBL experiments with muon storage rings . . . . . . . . . . . . . . . . 145

15 Conclusions $\quad 147$ 


\section{Introduction}

The neutrino is among one of the most fascinating elementary particles. There are three different neutrino species (flavours) in nature: $\nu_{e}, \nu_{\mu}$, and $\nu_{\tau}$ [1], partners of the corresponding charged leptons: $e, \mu$ and $\tau$. The neutrino has no electric charge, it interacts (very rarely) with matter only through weak interaction and, if massive, its mass is presumably very small: at least one hundred thousand times smaller than the electron mass, as in the case of the $\nu_{e}$.

The possibility of a non-vanishing neutrino-mass is an intriguing question in the present scenario of particle physics. On one hand, there are no fundamental principles for the neutrino to be massless; on the other hand, a massive neutrino would indicate the existence of physics beyond the Standard Model of the elementary particles, hence representing a fundamental milestone in particle physics.

The neutrino mass could also have profound implications for cosmology and astrophysics, possibly contributing to the explanation of the Dark Matter puzzle, namely the fact that most of the mass of the Universe is invisible [2]. This could influence our knowledge of the evolution of the Universe. Given the extremely large number of neutrinos in the Universe, even a tiny mass would have important consequences.

Most likely, the neutrino mass is too small to be within reach of direct measurements based on kinematical methods [3-6]. One way to measure it is to search for processes which may only occur if the neutrino has a mass, even very tiny. Neutrino oscillation, originally postulated by B. Pontecorvo in the late fifties $[7,8]$ and then by Z. Maki et al. [9], is one of these processes. The present description of the phenomenon in terms of weak and mass eigenstates was given by V. Gribov and B. Pontecorvo in the sixties [10].

A neutrino of a given flavour, $\nu_{e}, \nu_{\mu}$, or $\nu_{\tau}$, produced in a weak interaction, during its travel may appear to undergo a conversion into another flavour. The conversion probability has an oscillatory behaviour (hence oscillation). In first approximation, oscillations occur between pairs of neutrino flavours and are described by two variables: the mixing parameter $\sin ^{2} 2 \theta$ (related to the oscillation amplitude) and the mass-squared difference between the two oscillating flavours $\Delta m^{2}$ (related to the oscillation frequency). The sensitivity of the experiments to the above parameters depends on the neutrino energy $E$ and on the distance $L$ (baseline) of the detector from the neutrino source.

Presently, there are three experimental indications for neutrino oscillations. The detected solar $\nu_{e}$ flux is about a factor two smaller than that predicted by the so called Standard Solar Model (SSM) [11]. Matter-enhanced oscillations, the so-called Mikheyev-Smirnov-Wolfenstein (MSW) effect [12,13], or vacuum oscillations (just-so) can explain this deficit [14]. The observed deficit of $\nu_{\mu}$ produced in the atmosphere by cosmic rays measured in the Super-Kamiokande [15] experiment constitutes a strong evidence for neutrino oscillations. The experimental results indicate a small $\Delta m^{2}\left(\sim 10^{-2}-10^{-3} \mathrm{eV}^{2}\right)$ and a large mixing angle $\left(\sin ^{2} 2 \theta>0.8\right)$. The result of the reactor experiment CHOOZ [16-18], which explored the same parameter region, together with Super-Kamiokande itself excludes the possibility of $\nu_{\mu} \leftrightarrow \nu_{e}$ oscillations down to the $\Delta \mathrm{m}^{2}$ level of $10^{-3} \mathrm{eV}^{2}$ so favouring the hypothesis of $\nu_{\mu} \leftrightarrow \nu_{\tau}$ (or more exotically $\nu_{\mu} \leftrightarrow \nu_{\text {sterile }}$ ) oscillations. In addition, the claim from the LSND accelerator experiment [19-22] suggests the existence of $\bar{\nu}_{\mu} \leftrightarrow \bar{\nu}_{e}$ oscillations with $\Delta m^{2} \sim 1 e V^{2}$. The parameter regions allowed by the above positive signals in two-flavour mixing scheme are shown in Fig. 1.

The next generation of solar neutrino experiments is expected to substantially contribute to the experimental scenario of neutrino mixing. SNO [23,24] will perform the measurement of the charged to neutral current event ratio. Both SNO and Super-Kamiokande will be able to observe effects that can discriminate between neutrino oscillation schemes, such as day-night and seasonal variations of the solar signal (which are 


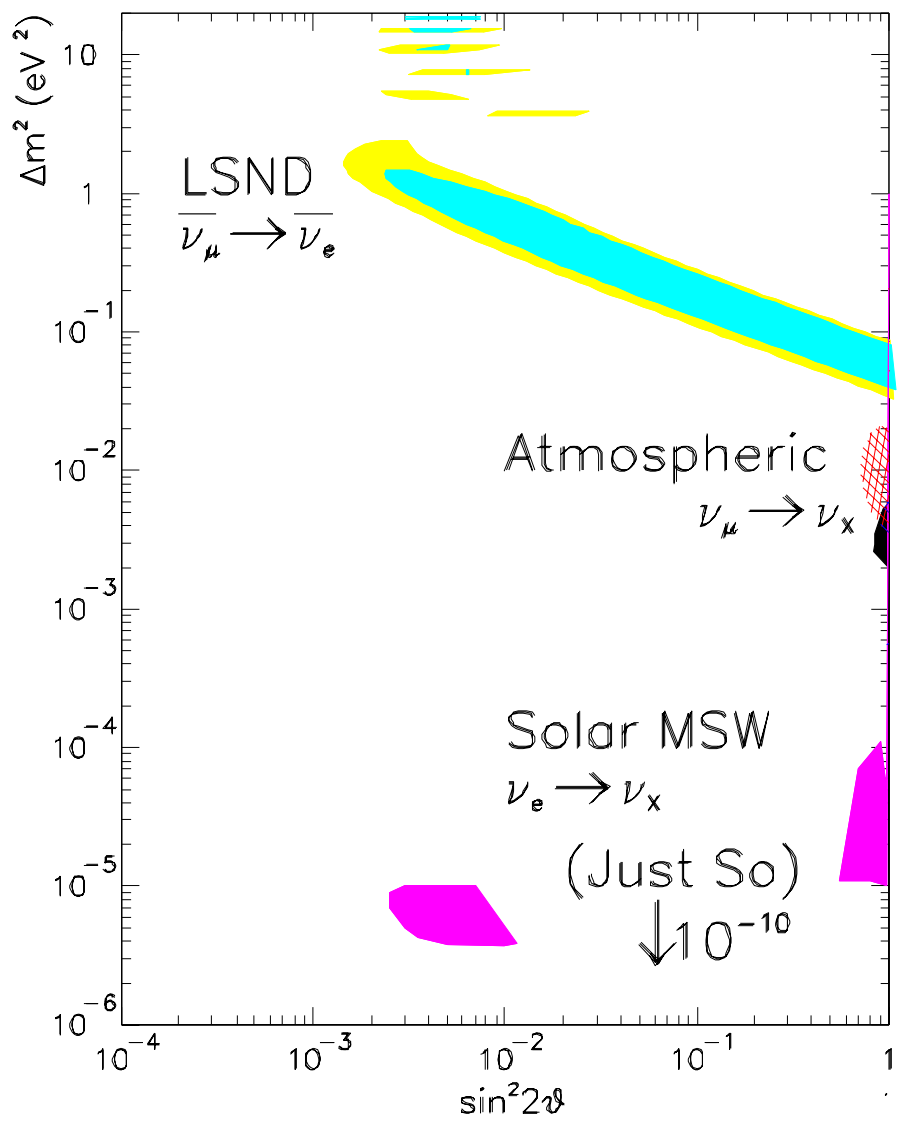

Figure 1: Positive signals of neutrino oscillations come from three sources: solar, atmospheric and accelerator neutrinos (LSND experiment). 
model independent) and spectrum distortion of the recoil electrons from neutrino interactions. The Borexino experiment [25-28] can address the problem of the apparent absence of neutrinos from ${ }^{7} \mathrm{Be}$ pointed out by model independent analyses of the solar neutrino data [14]. KAMLAND [29,30] will use reactor neutrinos to investigate MSW solutions of the solar neutrino deficit.

In the atmospheric neutrino field Super-Kamiokande will continue to take data aiming at a better determination of the mixing parameters and at the clarification whether the oscillations are $\nu_{\mu} \leftrightarrow \nu_{\tau}$ or $\nu_{\mu} \leftrightarrow \nu_{\text {sterile. }}$. In the meantime, a new experiment [31] has been proposed to check and possibly improve the Super-Kamiokande results by using a large magnetised calorimeter for the measurement of the oscillatory pattern of the atmospheric neutrino spectrum.

The subject of this review is the search for neutrino oscillations by neutrino beams from particle accelerators. Specific searches have been pursued in the past, some others are underway and more are planned at the future facilities. One usually refers to short (SBL) and long baseline (LBL) experiments, characterised by $L / E \sim 0.01-10 \mathrm{~km} / \mathrm{GeV}$ and $L / E \sim 100-10000 \mathrm{~km} / \mathrm{GeV}$, respectively.

The evidence for $\bar{\nu}_{\mu} \leftrightarrow \bar{\nu}_{e}$ oscillations reported by LSND is not confirmed by the KARMEN experiment [32] which only explored part of the parameter region allowed by LSND. The experiments MiniBooNE [33] and I216 [34] have been proposed to test the LSND results with higher sensitivity.

In the $\nu_{\mu} \leftrightarrow \nu_{\tau}$ channel, the two SBL CERN experiments CHORUS [35] and NOMAD [36] have shown $[37,38]$ no evidence for oscillations using only a fraction of the available data. CHORUS and NOMAD were proposed to search for oscillations in the parameter region relevant for the Dark Matter puzzle. They are sensitive to small mixing angles $\left(\sin ^{2} 2 \theta_{\mu \tau} \sim 10^{-4}\right)$ and relatively large $\Delta m^{2}\left(\mathcal{O}\left(10 e V^{2}\right)\right)$.

The region of oscillation parameters indicated by the atmospheric neutrino anomaly is hardly accessible by SBL searches. LBL experiments are needed to probe the small $\Delta m^{2}$ values favoured by the existing data. A new generation of experiments with $\nu_{\mu}$ beams produced by accelerators is being planned. The first LBL neutrino beam was commissioned in 1999 from KEK to the Super-Kamiokande detector about $250 \mathrm{~km}$ away [39-42]. At Fermilab, the LBL experiment MINOS [43] has been approved and will use a beam from Fermilab to the Soudan mine at $730 \mathrm{~km}$ distance to test the hypothesis of neutrino oscillations with $\Delta m^{2} \sim 10^{-2}-10^{-3} \mathrm{eV}^{2}$. Finally, several ideas for LBL experiments [44-50] have been proposed to exploit the CNGS beam from CERN to the Gran Sasso Laboratory $732 \mathrm{~km}$ away from the source. They will be largely discussed in this review. At present, the ICANOE [51] and OPERA [52,53] experiments are expected to run in the CNGS beam from the year 2005 with the aim of an appearance search for $\nu_{\mu} \leftrightarrow \nu_{\tau}$ oscillations.

Further studies will possibly be conducted by using neutrino beams from muon storage rings [54-57]. In this case high intensity beams can be produced from muon decay. The aim is to perform precision measurements of the neutrino mixing matrix elements and accurately determine the $\Delta m^{2}$ values. A new generation of LBL experiments could be designed for that purpose.

In the following, we first outline the neutrino oscillation mechanism. Different experimental methods for their detection are presented. The main experiments searching for neutrino oscillations by exploiting particle accelerator beams are reviewed. Finally, proposed and planned experiments are discussed, pointing to their discovery potential and to their role in the awaited clarification of the global scenario.

This review is necessarily not exhaustive. Experiments performed or planned in Europe are sometimes described in more detail or used as examples of specific oscillation searches. 


\section{Phenomenology of neutrino oscillations}

The idea of neutrino oscillations was developed by B. Pontecorvo in 1957 [7,8] and by Z. Maki et al. [9] in 1962. Pontecorvo, in particular, was originally led to propose the case of neutrino $\leftrightarrow$ anti-neutrino oscillations following the (wrong) rumour coming from a nuclear reactor experiment indicating the appearance of $\nu_{e}$ in a $\bar{\nu}_{e}$ reactor flux [58]. Z. Maki et al. first considered the possibility of neutrino flavour oscillations, presently studied. The present description of neutrino oscillations in terms of weak and mass eigenstates was then given by V. Gribov and B. Pontecorvo [10].

For a historical introduction to the theoretical concept of neutrino oscillations from early studies to the sixties we refer to $[58,59]$. In the following, a short description of the phenomenology of neutrino oscillations is presented. For detailed reviews on the subject we refer to [60-65].

\subsection{Neutrino oscillations in vacuum}

It can be shown that the introduction of a generic neutrino mass term in the mass Lagrangian leads to neutrino mixing, regardless whether Dirac or Majorana states are involved [66-68]. If neutrino mixing occurs and if all the mass eigenvalues are not identical, neutrino oscillations may take place. Under these assumptions, weak $\left(\nu_{e}, \nu_{\mu}, \nu_{\tau}\right)$ and mass $\left(\nu_{1}, \nu_{2}, \nu_{3}\right)$ eigenstates can be related

$$
\nu_{\alpha}=U_{\alpha i} \nu_{i} \quad \text { or } \quad \nu_{j}=U_{j \beta}^{\dagger} \nu_{\beta}
$$

where $\alpha, \beta=e, \mu, \tau$ and $i, j=1,2,3$. $U$ is an unitary matrix which connects the two sets of eigenvalues. The following formulae are derived by describing the oscillation pattern in terms of plane waves. This (usual) assumption implies that massive neutrinos travelling from the source have a common momentum $p_{i}=p$ and that neutrino masses $m_{i}$ are smaller than the corresponding $p_{i}$. This approach is not entirely correct

although practically adequate. A more general treatment must describe neutrinos as wave packets to take into account quantum mechanics effects (Section 2.2).

Let us consider an eigenstate $\nu_{\alpha}$ produced in a weak interaction reaction at the time $t=0$ at the position $x=0$. It can be expressed as a superposition of mass eigenstates with momentum $p_{i}$ and energy $E_{i}$

$$
\nu_{\alpha}(0)=\sum_{i} U_{\alpha i} \nu_{i}
$$

The space-time evolution is given by

$$
\nu_{i}(t)=\nu_{i}(0) e^{i\left(p_{i} x-E_{i} t\right)}
$$

It is essentially correct to assume the neutrino to be relativistic $(x \sim t)$

$$
E_{i}=\sqrt{p_{i}^{2}+m_{i}^{2}} \simeq p_{i}+\frac{m_{i}^{2}}{2 p_{i}}=p+\frac{m_{i}^{2}}{2 p}
$$

At the time $t$ (distance $x$ from the source) the eigenstate produced at $t=0$ is described by

$$
\nu_{\alpha}(x)=\sum_{i} U_{\alpha i} e^{i\left(p_{i} x-E_{i} x\right)} \nu_{i}(0)
$$


By using expression (4) and taking into account that in the relativistic limit $E \sim p$, one obtains

$$
\nu_{\alpha}(x)=\sum_{i} U_{\alpha i} e^{-i \frac{m_{i}^{2}}{2 E} x} \nu_{i}(0)
$$

For an experiment detecting neutrinos at the distance $x$ the probability to actually measure the weak eigenstate $\beta$ is

$$
P(\alpha \leftrightarrow \beta)=\left|\left\langle\nu_{\beta}(0) \mid \nu_{\alpha}(x)\right\rangle\right|^{2}=\left|\sum_{i} U_{\alpha i} e^{-i \frac{m_{i}^{2}}{2 E} x}\left\langle\nu_{\beta}(0) \mid \nu_{i}(0)\right\rangle\right|^{2}
$$

By using Eq. (2)

$$
P(\alpha \leftrightarrow \beta)=\left|\sum_{i} U_{\alpha i} U_{i \beta}^{\dagger} e^{-i \frac{m_{i}^{2}}{2 E} x}\right|^{2}=\sum_{i}\left|U_{\alpha i} U_{i \beta}^{\dagger}\right|^{2}+2 R e \sum_{i>j} U_{\alpha i} U_{i \beta}^{\dagger} U_{\beta j} U_{j \alpha}^{\dagger} e^{-i \frac{\left|m_{j}^{2}-m_{i}^{2}\right|}{2 E} x}
$$

The first term of Eq. (5) can be written as

$$
\sum_{i}\left|U_{\alpha i} U_{i \beta}^{\dagger}\right|^{2}=\left|\sum_{i} U_{\alpha i} U_{i \beta}^{\dagger}\right|^{2}-2 R e \sum_{i>j} U_{\alpha i} U_{i \beta}^{\dagger} U_{\beta j} U_{j \alpha}^{\dagger} \delta_{\alpha \beta}-2 R e \sum_{i>j}\left|U_{\alpha i} U_{i \beta}^{\dagger} U_{\beta j} U_{j \alpha}^{\dagger}\right| e^{i \phi_{\alpha \beta i j}}
$$

where $\phi_{\alpha \beta i j}$ is the phase of the product $U_{\alpha i} U_{i \beta}^{\dagger} U_{\beta j} U_{j \alpha}^{\dagger}$. Then the probability becomes

$$
P(\alpha \leftrightarrow \beta)=\delta_{\alpha \beta}+2 R e \sum_{i>j}\left|U_{\alpha i} U_{i \beta}^{\dagger} U_{\beta j} U_{j \alpha}^{\dagger}\right|\left[\cos \left(\frac{\left|m_{j}^{2}-m_{i}^{2}\right|}{2 E} x-\phi_{\alpha \beta i j}\right)-\cos \phi_{\alpha \beta i j}\right]
$$

If $\mathrm{CP}$ is conserved, the matrix $U$ is real and the previous expression may be written as

$$
P(\alpha \leftrightarrow \beta)=\delta_{\alpha \beta}-4 \sum_{i>j}\left|U_{\alpha i} U_{i \beta}^{\dagger} U_{\beta j} U_{j \alpha}^{\dagger}\right| \sin ^{2}\left[\frac{\left|m_{j}^{2}-m_{i}^{2}\right|}{4 E} x\right]
$$

One can define $\Delta m^{2} \equiv\left|m_{j}^{2}-m_{i}^{2}\right|$. The oscillations then occur if $\Delta m^{2} \neq 0$. The argument of the sinus squared may be conveniently expressed as

$$
\frac{\Delta m^{2}}{4 E} x \rightarrow 1.267 \frac{\Delta m^{2}\left(e V^{2}\right) L(k m)}{E(G e V)}
$$

$E$ being the neutrino energy and $L$ the distance travelled during the time $t$. The oscillation length $\lambda$ is defined as

$$
\lambda=\frac{\pi E}{1.267 \Delta m^{2}}
$$




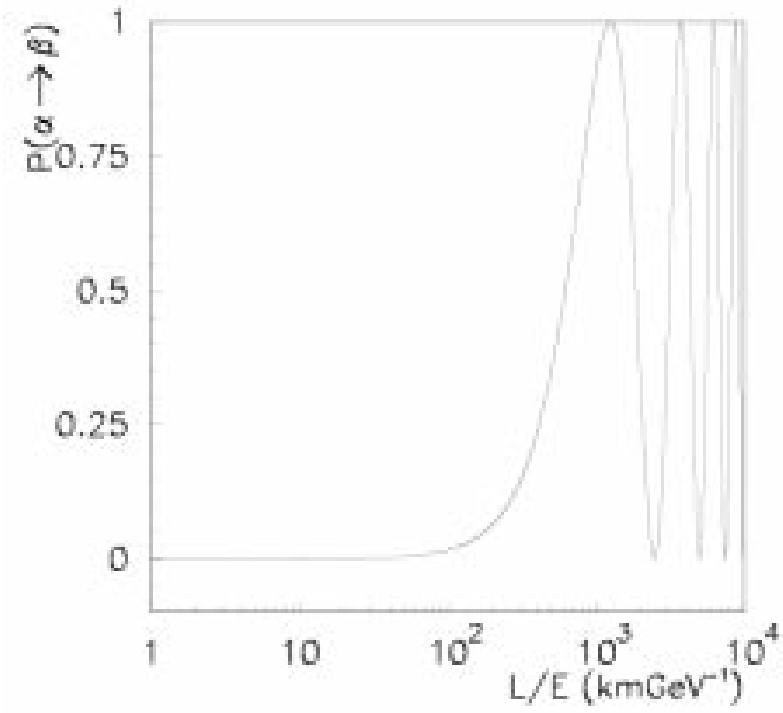

Figure 2: Oscillation probability as a function of $L / E$ for $\Delta m^{2}=0.001 \mathrm{eV}^{2}$ and full mixing.

The oscillation probability $P(\alpha \leftrightarrow \beta)$ has its first maximum at $L=\lambda / 2$. From Eq. (8) we see that, for a given $\Delta m^{2}$, the detectable signal is a function of the ratio $L / E$ (Fig. 2). Therefore, the sensitivity of an experiment to $\Delta m^{2}$ depends on the neutrino energy and on the distance source-detector (baseline).

We observe that if the CPT theorem holds $P(\alpha \leftrightarrow \beta)=P(\bar{\beta} \leftrightarrow \bar{\alpha})$ and that under the assumption of CP invariance $P(\alpha \leftrightarrow \beta)=P(\bar{\alpha} \leftrightarrow \bar{\beta})$. A pedagogical introduction on the implications of $\mathrm{CP}, \mathrm{T}$ and $\mathrm{CPT}$ symmetries for neutrino oscillations is given in [69] and references therein.

Neutrino oscillation experiments can be performed by using various neutrino sources with different energy spectra and baselines: neutrinos from the Sun $\left(\nu_{e}\right)$, from the interaction of cosmic rays with the atmosphere $\left(\nu_{\mu}, \bar{\nu}_{\mu}, \nu_{e}\right.$ and $\left.\bar{\nu}_{e}\right)$ and from terrestrial sources, i.e. from nuclear reactors and accelerators $\left(\nu_{\mu}, \bar{\nu}_{\mu}, \nu_{e}\right.$ and $\left.\bar{\nu}_{e}\right)$. In Table 1, the $L$ and $E$ values characteristics of the different types of experiments are listed together with the values of $\Delta m^{2}$ corresponding to the first oscillation maximum.

Experiments can also be classified as disappearance and appearance experiments, according to the method adopted to search for neutrino oscillations. For disappearance experiments the main component of the neutrino beam is measured at a given distance $L$ from the source. The occurrence of neutrino oscillations can be inferred from the reduction of the measured flux compared with the value expected in the absence of oscillations. Appearance experiments exploit sources producing predominantly one neutrino flavour $\nu_{\alpha}$ and search for the appearance of the eigenstate $\nu_{\beta}$ which is in principle not present in the beam. These issues are discussed in Section 3. 


\begin{tabular}{||c|c|c|c||}
\hline Neutrino source & Baseline $L(\mathrm{~km})$ & Energy $(\mathrm{GeV})$ & $\begin{array}{c}\Delta \mathrm{m}^{2}\left(\mathrm{eV}^{2}\right) \\
\text { (first maximum) }\end{array}$ \\
\hline \hline Sun & $10^{8}$ & $10^{-3}$ & $10^{-11}$ \\
\hline Atmosphere (from zenith) & 10 & 1 & $10^{-1}$ \\
\hline Atmosphere (from nadir) & $10^{4}$ & 1 & $10^{-4}$ \\
\hline Nuclear reactors & $10^{-2}$ & $5 \times 10^{-3}$ & 0.5 \\
\hline Accelerators (short baseline) & 1 & 20 & 20 \\
\hline Accelerators (long baseline) & 1000 & 20 & $10^{-2}$ \\
\hline \hline
\end{tabular}

Table 1: $\Delta m^{2}$ sensitivity domain for different neutrino sources.

\subsection{Quantum mechanics of neutrino oscillations}

As shown in the previous Section neutrino oscillations originate from the interference between the mass eigenstates of the neutrino wave function. This interference only occurs if there is coherence among the eigenstates and if no measurements perturb the state of the system. A thorough analysis of the neutrino oscillation quantum-mechanics can be found in [70,71]. Here we only summarise the main results.

Suppose we perform a $\nu_{\mu} \leftrightarrow \nu_{e}$ oscillation experiment where $\nu_{\mu}$ are produced through the reaction $\pi^{+} \rightarrow \mu^{+}+\nu_{\mu}$ and to observe a positive signal (i.e. an excess of $\nu_{e}$ interactions). The oscillatory $x$ dependence predicted by Eq. (8) must be observed. We then assume to have a detector which measures the pion and muon momenta for each event. If the measurement is so precise that the error $\left(\delta m_{\nu}^{2}\right)$ on $m_{\nu}^{2}$ is smaller than $\left|m_{i}^{2}-m_{j}^{2}\right|$ (with $i$ and $j$ two mass eigenstates) one would know for each event the eigenstate actually involved. The oscillatory pattern would then be washed out. Therefore, the number of $\nu_{e}$ interactions becomes proportional to the probability for a $\pi$ to produce a $\nu_{\mu}$ with mass eigenstate $\nu_{i}$ $\left(U_{\mu i}^{2}\right)$ times the probability for the $\nu_{i}$ to produce a $\nu_{e}$ interaction $\left(U_{e i}^{2}\right)$. Only a single physical neutrino would contribute to any given event and there would be no coherent contribution from several $\nu_{i}$.

One can then argue why the oscillatory pattern does disappear. The answer is given by the uncertainty principle. Given $m_{\nu}^{2}=E^{2}-p^{2}$ and $\delta m_{\nu}^{2} \ll\left|m_{i}^{2}-m_{j}^{2}\right|$, if one assumes that the errors on $E$ and $p$ are uncorrelated

$$
\delta m_{\nu}^{2}=\sqrt{(2 E)^{2}(\delta E)^{2}+(2 p)^{2}(\delta p)^{2}} \simeq 2 p \delta p<\left|m_{i}^{2}-m_{j}^{2}\right|
$$

From the uncertainty principle $\delta x \delta p \geq 1$. Therefore

$$
\delta x \geq \frac{1}{\delta p} \geq \frac{2 p}{\left|m_{i}^{2}-m_{j}^{2}\right|} \text { or } \delta x \geq \frac{2 \times 1.267}{\pi} \lambda
$$

i.e. the uncertainty on the neutrino production point is larger than the oscillation length and the oscillation pattern disappears. The minimum value for the neutrino momentum-spread is also determined by the uncertainty principle

$$
\delta p \geq \frac{1}{\delta x} \geq \frac{\pi}{2 \times 1.267} \frac{1}{\lambda}
$$


A neutrino wave packet can be considered as the sum of amplitudes corresponding to several neutrinos each with a well defined mass eigenvalue. For a given momentum, the various components of the wave function travel with different velocities. The corresponding amplitude may interfere if coherence is kept. Two amplitudes become spatially separated (and the coherence is lost) if their velocities are so different that, at the time $t$, their relative distance becomes larger than the initial size of the wave packet. The different components $\nu_{i}$ of a given weak interaction eigenstate have velocities

$$
\beta_{i}=\sqrt{1-\left(\frac{m_{i}}{E}\right)^{2}} \simeq 1-\frac{1}{2}\left(\frac{m_{i}}{E}\right)^{2}
$$

Therefore, the difference in the velocities of two mass eigenstate wave-packets leads to a separation $\Delta d$ after the distance $x$

$$
\Delta d \simeq t \Delta \beta \simeq \frac{x \Delta m^{2}}{2 E^{2}}
$$

If the wave packet of the emitted neutrino has dimension $d$ along the direction of propagation, one obtains the following coherence condition

$$
\Delta d \simeq \frac{x \Delta m^{2}}{2 E^{2}}<d \quad \text { or } \quad x<\frac{2 \times 1.267}{\pi} \lambda E d
$$

The coherence is preserved if the ratio between the travelled distance and the oscillation-length is smaller than $E d$.

For example, the size of the source for reactor neutrinos is $\sim 1 \mathrm{~m}, L \sim 10 \mathrm{~m}$ and $E \sim 1 \mathrm{MeV}$. For $\Delta m^{2}=1 e V^{2} x<2 \times 10^{12} \mathrm{~m}$. Since $L \ll 10^{12} \mathrm{~m}$ the coherence is preserved. This is also valid for atmospheric neutrinos which originate from pion and muon decay. For solar neutrinos the size of the wave packet is estimated to be $10^{-8} \mathrm{~m}$ [72]. By using the typical energy value of $1 \mathrm{MeV}$ one finds $x<10^{4} \mathrm{~m}$. This length has to be compared with the Sun-Earth distance $\left(1.5 \times 10^{11} \mathrm{~m}\right)$. In this case the coherence condition is not satisfied and the oscillatory pattern cannot be observed anymore. Only a reduction of the neutrino flux can be detected.

\subsection{Two-flavour oscillations}

When dealing with experimental results, the neutrino oscillation formalism is usually expressed by assuming that oscillations essentially occur among pairs of weak interaction eigenstates. For example, the flavour eigenstates $\nu_{e}$ and $\nu_{\mu}$ and the mass eigenstates $\nu_{1}$ and $\nu_{2}$ take part in the determination of the oscillation probability. The mixing matrix becomes

$$
U=\left(\begin{array}{cc}
\cos \theta & \sin \theta \\
-\sin \theta & \cos \theta
\end{array}\right)
$$

and the probability $P\left(\nu_{\mu} \leftrightarrow \nu_{e}\right)$ given by Eq. (8) reduces to

$$
P\left(\nu_{\mu} \leftrightarrow \nu_{e}\right)=\sin ^{2} 2 \theta \sin ^{2}\left(1.267 \frac{\Delta m^{2}\left(e V^{2}\right) L(k m)}{E(G e V)}\right)
$$


This simple expression allows us to make some considerations on the oscillatory pattern of $P\left(\nu_{\mu} \leftrightarrow \nu_{e}\right)$. As one can see from Fig. 2, if $\Delta m^{2} \gg E / L$ the frequency of the oscillations is so high that it becomes difficult to distinguish two consecutive maxima because of the limited experimental resolution and of the typical broad neutrino energy spectra. The oscillatory dependence on $x$ is washed out and the average value of $\sin ^{2}\left(1.267 \Delta m^{2} L / E\right)$ is observed. The oscillation probability thus only depends on the mixing angle

$$
P\left(\nu_{\mu} \leftrightarrow \nu_{e}\right)=\frac{1}{2} \sin ^{2} 2 \theta
$$

Conversely, if $\Delta m^{2} \ll E / L$,

$$
P\left(\nu_{\mu} \leftrightarrow \nu_{e}\right) \simeq \sin ^{2} 2 \theta\left(1.267 \frac{\Delta m^{2}\left(e V^{2}\right) L(k m)}{E(G e V)}\right)^{2}
$$

Also in this case the oscillatory pattern is not visible and the oscillation probability depends on $\left(\Delta m^{2}\right)^{2}$.

From Eq. (9) it follows that if an experiment measures the oscillation probability $P\left(\nu_{\alpha} \leftrightarrow \nu_{\beta}\right)$ a relation between $\sin ^{2} 2 \theta$ and $\Delta m^{2}$ is established for a given average $E$ and $L$. For a positive search, the parameter domain allowed by the experiment is represented by a zone in the $\Delta m^{2}-\sin ^{2} 2 \theta$ plane (dashed area in Fig. 3 ) whose extension is given by the experimental resolution in the determination of the oscillation probability.

In the case of a negative search only an upper limit (at a given confidence level (C.L.)) on the oscillation probability $\left(P_{\min }\right)$ can be given. Since $P$ is a function of $\Delta m^{2}$ and $\sin ^{2} 2 \theta$ (Eq. (9)) $P_{\min }$ is represented by a curve, the so-called exclusion plot. This curve defines a region in the plane $\Delta m^{2}-\sin ^{2} 2 \theta$ where neutrino oscillations are excluded at a given C.L. An example of the exclusion plot is shown in Fig. 3. From Eq. (10) the minimum excluded value for $\sin ^{2} 2 \theta\left(\sin ^{2} 2 \theta_{\text {min }}\right)$ at high $\Delta m^{2}$ amounts to $2 P_{\text {min }}$. From Eq. (11) the lowest $\Delta m^{2}\left(\Delta m_{\min }^{2}\right.$ in Fig. 3) which can be probed (for full mixing, i.e. $\sin ^{2} 2 \theta=1$ ) is equal to approximately $E \sqrt{P_{\min }} /(1.267 L)$.

In Section 11 we show how neutrino oscillations can be described in a more rigorous way assuming that mixing occurs among all neutrino flavours (three-flavour scheme).

\section{Detection of neutrino oscillations}

As discussed in the previous Sections there are essentially two strategies for the detection of neutrino oscillations: those employed by disappearance and appearance experiments.

In the first case the main experimental requirement is a neutrino source with well-known intensity. Hence, the sensitivity of disappearance experiments is limited by systematic uncertainties on the knowledge of the neutrino flux. For this reason the measurement of the flux can profit from another detector placed at a distance $L^{\prime}$ from the source smaller than $L$. This is then called a near detector. The experiment has to look for (small) deviations of $P\left(\nu_{\alpha} \leftrightarrow \nu_{\alpha}\right)$ from 1. Disappearance experiments do not detect neutrinos of the flavour $\nu_{\beta}$ due to $\nu_{\alpha} \leftrightarrow \nu_{\beta}$ oscillations. They can only look for $\nu_{\alpha} \leftrightarrow \nu_{x}$ oscillations where $x$ can be any neutrino species. The experiment can be carried out even if the fraction of $\nu_{\beta}$ in the beam with respect to the main component $\nu_{\alpha}$ is not negligible.

Appearance experiments have to be very efficient in the detection of $\nu_{\beta}$ and in background rejection. Their sensitivity is limited by the uncertainty in the contamination of $\nu_{\beta}$ to the main $\nu_{\alpha}$ flux and by the background rejection power. 


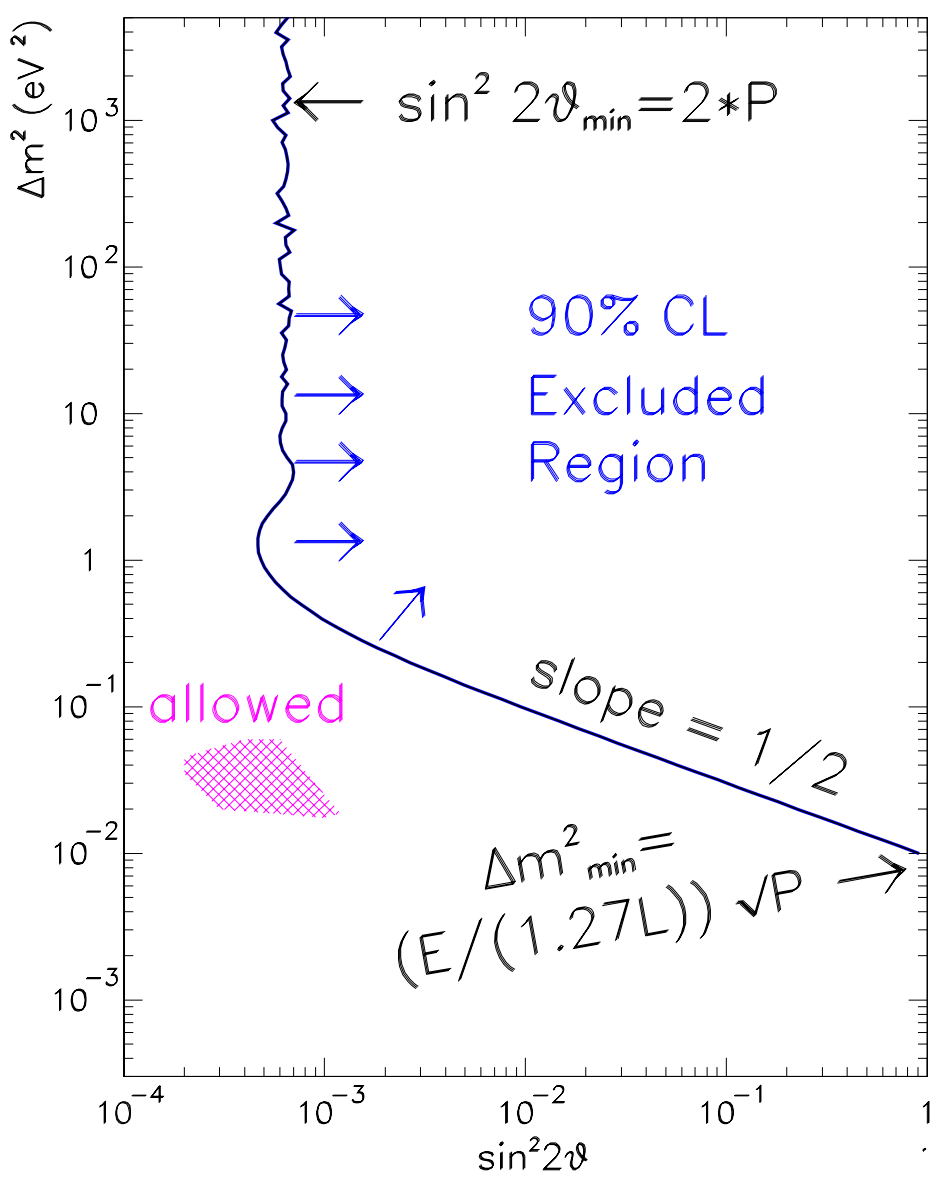

Figure 3: The exclusion plot obtained by generic neutrino oscillation experiments in a two-flavour scheme. The region to the right of the solid line is excluded at the $90 \%$ confidence level. The shaded blob represents an allowed region corresponding to a positive search [73]. 


\subsection{Disappearance experiments}

\subsubsection{Near/far test for disappearance experiments}

We consider two detectors located at distances $L_{n}$ (near) and $L_{f}$ (far) from the neutrino source, respectively. $N_{n}\left(N_{f}\right)$ is the number of expected interactions in the near (far) detector and $P_{n}\left(\nu_{\alpha} \leftrightarrow \nu_{\beta}\right)\left(P_{f}\left(\nu_{\alpha} \leftrightarrow \nu_{\beta}\right)\right)$ the probability that oscillations occur at a distance $L_{n}\left(L_{f}\right)$.

In the absence of oscillations, one can define the double ratio $R_{f / n}$ as

$$
R_{f / n} \equiv \frac{\left(N_{f} / N_{n}\right)^{o b s}}{\left(N_{f} / N_{n}\right)^{e x p}}
$$

By observed events we intend charged current (CC) interactions of $\nu_{\alpha}$ neutrinos. It is in fact impossible to distinguish between neutral current (NC) interactions induced by different neutrino flavours.

If neutrino oscillations occur the above ratio becomes

$$
r_{f / n}=\frac{\left(N_{f}\left(1-P_{f}\right) / N_{n}\left(1-P_{n}\right)\right)^{o b s}}{\left(N_{f} / N_{n}\right)^{e x p}} \equiv \frac{\left(N_{f}^{o s c} / N_{n}^{o s c}\right)^{o b s}}{\left(N_{f} / N_{n}\right)^{e x p}}
$$

If $\phi_{\alpha}(E, x)$ is the neutrino flux at the distance $x$ from the source and $E$ the neutrino energy, the number of events measured in the near (far) detector is

$$
n_{n(f)}(E)=S_{n(f)} \int_{L_{n(f)}}^{L_{n(f)}+l_{n(f)}} \sigma_{c c}(E) \varepsilon(E) \phi_{\alpha}(E, x) d x
$$

where $S_{n(f)}$ is a factor which takes into account the detector parameters (mass, geometry, baseline and source intensity), $\sigma_{c c}(E)$ is the neutrino CC cross-section, $\varepsilon(E)$ is the detection efficiency and $l_{n(f)}$ is the detector length along the beam direction. In order to compute the expected total rate one has to integrate over the neutrino energy spectrum. In the absence of oscillations the expected rates are

$$
N_{n(f)}=S_{n(f)} \int n_{n(f)}(E) d E
$$

Conversely, if neutrino oscillations occur with parameters $\Delta m^{2}$ and $\sin ^{2} 2 \theta$ and the lepton $\beta$ produced in the $\mathrm{CC}$ interaction of the $\nu_{\beta}$ cannot decay into the lepton $\alpha$, the expected rates are

$$
N_{n(f)}^{o s c}=S_{n(f)} \int n_{n(f)}(E)\left(1-P_{n(f)}\right) d E
$$

If the lepton $\beta$ can decay into $\alpha$, the previous formula is slightly modified in

$$
N_{n(f)}^{o s c}=S_{n(f)} \int\left\{n_{n(f)}(E)\left(1-P_{n(f)}\right)+n_{n(f)}(E) P_{n(f)} \frac{\sigma_{\beta}(E)}{\sigma_{\alpha}(E)} B R_{\beta \alpha}\right\} d E
$$


where $B R_{\beta \alpha}$ is the branching ratio for the decay $\beta \rightarrow \alpha$. In the following, we assume $B R_{\beta \alpha}=0$.

The occurrence of oscillations is detected by comparing the measured ratio $r_{f / n}$ with the expected one $R_{f / n}$. This test, however, depends on the uncertainties due to statistical fluctuations. If the difference $r_{f / n}-R_{f / n}$ is statistically consistent with the absence of oscillations, an upper limit can be set on the oscillation probability. If the experiment statistics is large enough the distribution of the measured ratios is a Gaussian centered to the expected value

$$
\left|r_{f / n}-R_{f / n}\right|<s \sigma_{R}
$$

With $s=1.64$ one defines the $90 \%$ C.L. upper limit on $r_{f / n}$, being $\sigma_{R}=R_{f / n} \sqrt{1 / N_{f}+1 / N_{n}}$ the

error on $\left|r_{f / n}-R_{f / n}\right|$. If also a systematic error is taken into account $\sigma_{R}$ takes a slightly different form $\left(\sigma_{R}=R_{f / n} \sqrt{1 / N_{f}+1 / N_{n}+(s y s t)^{2}}\right)$. With simple algebra it is possible to write Eq. (12) as

$$
|\overbrace{\int \frac{n_{n}(E) P_{n} d E}{\int n_{n}(E) d E}}^{a}-\overbrace{\frac{\int n_{f}(E) P_{f} d E}{\int n_{f}(E) d E}}^{b}|=\left|\frac{1.64 \sigma_{R}}{R_{f / n}}\left(1-\frac{\int n_{n}(E) P_{n} d E}{\int n_{n}(E) d E}\right)\right|
$$

One can then split Eq. (13) in two. For $a>b$

$$
\left(1+\frac{1.64 \sigma_{R}}{R_{f / n}}\right) \frac{\int n_{n}(E) P_{n} d E}{\int n_{n}(E) d E}-\frac{\int n_{f}(E) P_{f} d E}{\int n_{f}(E) d E}=\frac{1.64 \sigma_{R}}{R_{f / n}}
$$

while, if $a<b$

$$
\left(\frac{1.64 \sigma_{R}}{R_{f / n}}-1\right) \frac{\int n_{n}(E) P_{n} d E}{\int n_{n}(E) d E}+\frac{\int n_{f}(E) P_{f} d E}{\int n_{f}(E) d E}=\frac{1.64 \sigma_{R}}{R_{f / n}}
$$

The excluded region at the $90 \%$ C.L. is obtained by solving Eqs. (14) and (15) as a function of $\Delta m^{2}$ and $\sin ^{2} 2 \theta$. In the limit $\Delta m^{2} \gg E / L_{n(f)}$ Eqs. (14) and (15) reduce to a trivial equality and the experiment is no longer sensitive to neutrino oscillations. The same conclusion is reached when $\Delta m^{2} \ll E / L_{n(f)}$.

\subsubsection{Energy measurement of $\nu_{\mu} \mathrm{CC}$ events}

At a given distance from the source, neutrino oscillations manifest themselves by the modulation of the energy distribution of neutrino interactions in a detector as compared with the distribution predicted in the absence of oscillations.

For example, consider the case of a detector placed at a distance of $1000 \mathrm{~km}$ from the source. The mean energy of the interacting neutrinos is $\left\langle E_{\nu}\right\rangle \sim 20 \mathrm{GeV}$. The expected energy distribution of $\nu_{\mu} \mathrm{CC}$ events with and without oscillations is shown in Fig. 4. The oscillation parameters $\Delta m^{2}=0.1 e V^{2}$ and $\sin ^{2} 2 \theta=1$ are assumed together with an ideal detector with unit detection efficiency [74]. 

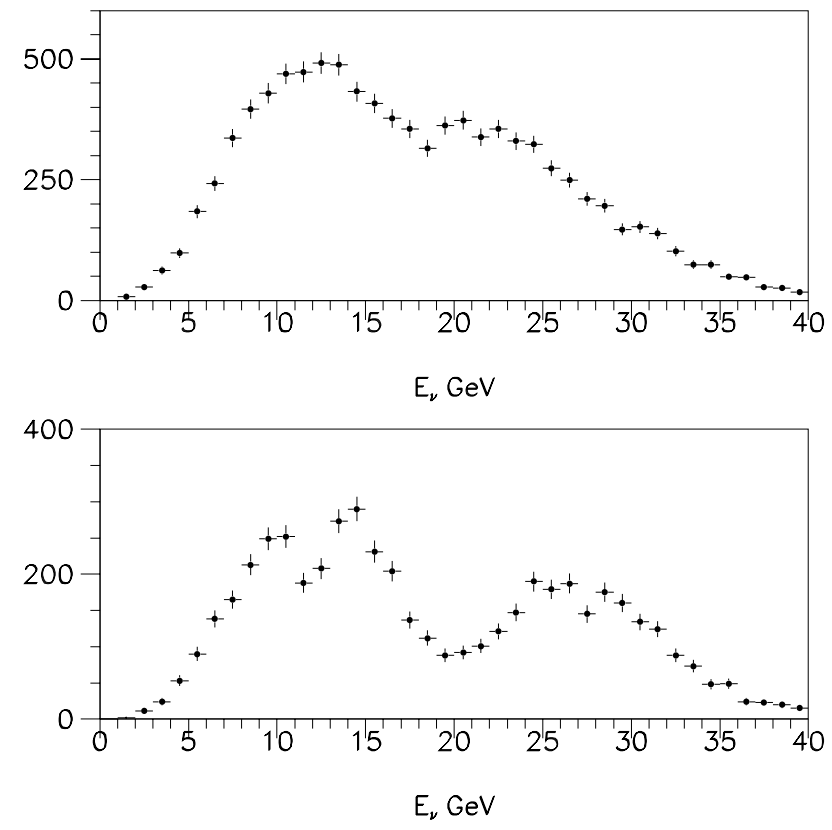

Figure 4: Example of the effect of neutrino oscillations on the observed neutrino energy spectrum. The mean energy of $\nu_{\mu}$ CC events is $\left\langle E_{\nu}\right\rangle \sim 20 \mathrm{GeV}$. Top Figure: no oscillations; bottom Figure: oscillations occur with $\Delta m^{2}=0.1 \mathrm{eV}^{2}$ and $\sin ^{2} 2 \theta=1$. An ideal detector located at $\sim 1000 \mathrm{~km}$ away from the neutrino source is assumed. 
For such an experiment a broad spectrum favours the observation. The sensitivity to small $\Delta m^{2}$ improves with increasing the baseline $L$, lowering the beam energy and reducing the uncertainty on the expected unoscillated neutrino energy distribution. The highest detectable $\Delta m^{2}$ is limited by the detector resolution.

The observation of a modulation in the energy spectrum can provide direct evidence for neutrino oscillations as well as a measurement of $\Delta m^{2}$. Moreover, it has one important advantage as compared with a disappearance test based on event counting: one does not need to know the total number of expected events used as normalisation. The normalised statistics $Z$ [74] is

$$
Z_{f}\left(\Delta m^{2}\right)=\frac{1}{N_{f}} \sum_{i=1}^{N_{f}} \cos \left(2.54 \frac{\Delta m^{2} L}{E_{i}}\right)
$$

where $N_{f}$ is the number of detected CC events in the far detector. $Z_{f}\left(\Delta m^{2}\right)$ is then compared with the expected value in the absence of oscillation $Z_{n}\left(\Delta m^{2}\right)$. A statistically significant difference between $Z_{f}\left(\Delta m^{2}\right)$ and $Z_{n}\left(\Delta m^{2}\right)$ may reveal the occurrence of oscillations. The difference is maximal for $\Delta m^{2}=$ $\Delta m_{0}^{2}$, the true value of $\Delta m^{2}$. This test yields a direct measurement of $\Delta m^{2} . Z_{n}\left(\Delta m^{2}\right)$ must be predicted by using the energy spectrum of $\mathrm{CC}$ events in the near detector and extrapolating the flux to the far detector.
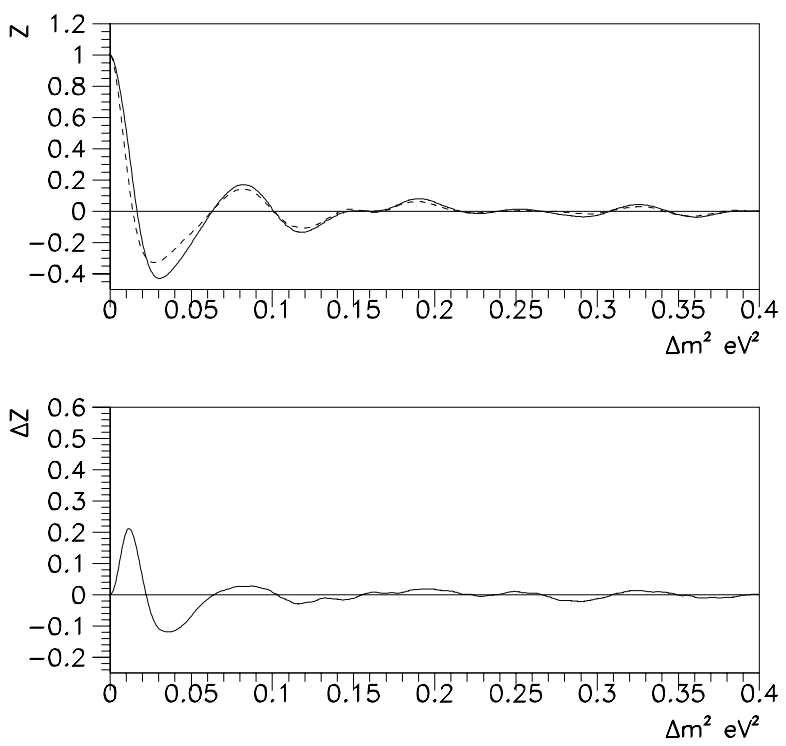

Figure 5: Top Figure: $Z_{f}\left(\Delta m^{2}\right)$ (continuous line) and $Z_{n}\left(\Delta m^{2}\right)$ (dashed line) with oscillation parameters $\Delta m_{0}^{2}=0.01 \mathrm{eV}^{2}$ and $\sin ^{2} 2 \theta=1$. Bottom Figure: difference between the two above curves.

Fig. 5 shows an example of the oscillation signatures which can be obtained with this method for $\Delta m_{0}^{2}=0.01 \mathrm{eV}^{2}$ and full mixing [74]. In this case $Z_{f}\left(\Delta m^{2}\right)-Z_{n}\left(\Delta m^{2}\right)$ is maximal at $\Delta m_{0}^{2}$. It can be shown that for a detector with perfect energy resolution the height of the maximum at $\Delta m^{2}=\Delta m_{0}^{2}$ is related to $\sin ^{2} 2 \theta$ by the expression 


$$
Z_{f}\left(\Delta m^{2}\right)-Z_{n}\left(\Delta m^{2}\right)=\frac{\sin ^{2} 2 \theta}{2}\left(\frac{1-2 Z_{n}^{2}\left(\Delta m^{2}\right)+Z_{n}\left(2 \Delta m^{2}\right)}{2-\sin ^{2} 2 \theta\left(1-Z_{n}\left(\Delta m^{2}\right)\right)}\right)
$$

$\sin ^{2} 2 \theta$ can be derived for large $\Delta m^{2}$ from

$$
Z_{f}\left(\Delta m^{2}\right)-Z_{n}\left(\Delta m^{2}\right)=\frac{\sin ^{2} 2 \theta}{4-2 \sin ^{2} 2 \theta}
$$

The main systematic errors are those due to the extrapolation of the energy dependence of the neutrino flux from the near to the far detector, and to energy scale uncertainties, e.g. the energy calibration of the detector.

The result of the application of the above method is depicted in Fig. 6. The three sensitivity curves shown in the Figure refer, respectively, to an ideal detector, to a detector with a $5 \%$ relative error in the muon momentum measurement and $\sigma(E) / E=70 \% / \sqrt{E(G e V)}+8 \%$ hadronic energy resolution, and to the case where the effect of a calibration systematic error is taken into account [74].

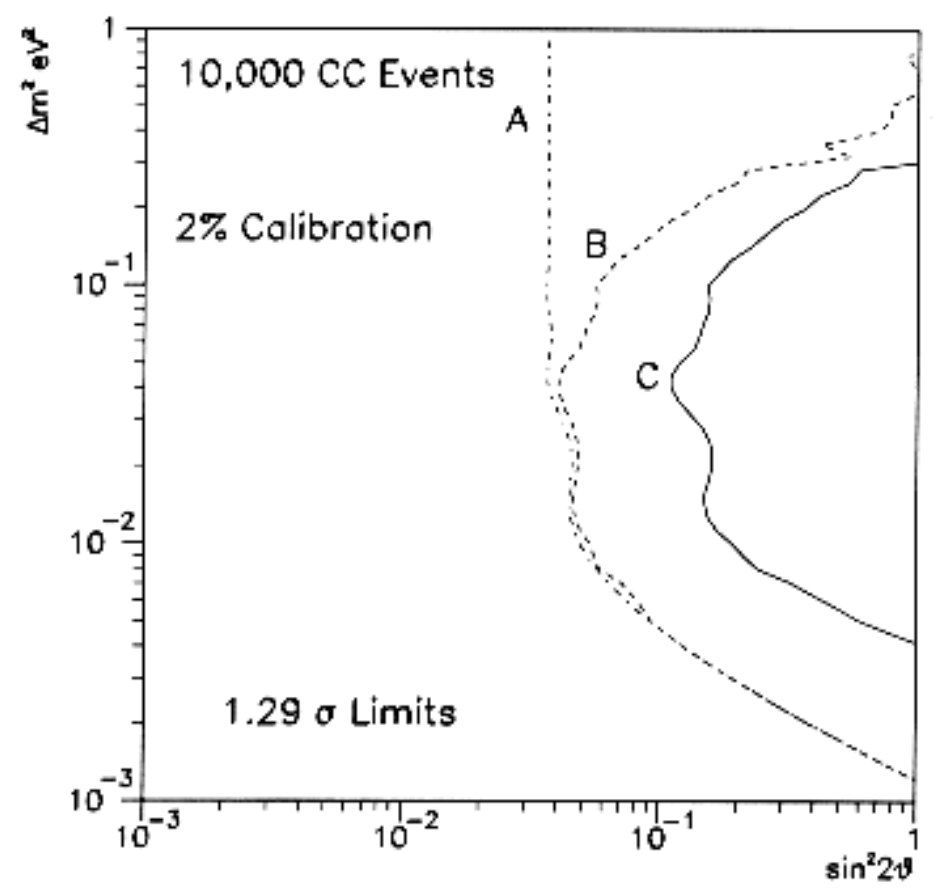

Figure 6: Sensitivity limits (90\% C.L.) which can be achieved by an experiment employing the method described in the text (energy measurement). Curve A: an ideal detector is assumed; curve B: finite detector resolution is assumed (see text); curve C: an additional $2 \%$ calibration error is included.

\section{$3.2 \quad$ Appearance experiments}

The detector of a $\nu_{\alpha} \leftrightarrow \nu_{\beta}$ appearance experiment must be sensitive to the interactions of $\nu_{\beta}$ neutrinos. Appearance experiments can be further subdivided into two classes: direct appearance and indirect ap- 
pearance searches. The latter includes experiments where one can separate $\nu_{\beta}$ induced interactions from background on a statistical basis, looking for deviations from expectations of the distribution of kinematical variables, of the $\mathrm{NC} / \mathrm{CC}$ cross-section ratio or of other quantities. In direct appearance experiments neutrino oscillations are detected through the identification of the lepton $\beta$ produced in $\nu_{\beta} \mathrm{CC}$ interactions, with low background and hence (ideally) on an event-by-event basis.

Since the appearance manifests itself through the CC interaction of the $\nu_{\beta}$ with the experimental target, the energy of the neutrino must be high enough to produce its lepton partner. This sets a lower limit on the neutrino energy. For example, a high energy beam is needed for $\nu_{\mu} \leftrightarrow \nu_{\tau}$ appearance experiments since the kinematical threshold to produce a $\tau$ is $\sim 3.5 \mathrm{GeV}$.

Particle accelerators provide $\nu_{\mu}$ beams. In the following, we outline general methods which can be used for appearance experiments searching for $\nu_{\mu} \leftrightarrow \nu_{\tau}$ oscillations. An example of a $\nu_{\mu} \leftrightarrow \nu_{e}$ appearance experiment is also presented. More details are given together with the description of specific experiments.

\subsubsection{NC/CC test for $\nu_{\mu} \leftrightarrow \nu_{\tau}$ oscillations}

The measurement of the NC/CC ratio provides a test for the appearance of a new flavour as it produces an apparent reduction of the CC sample and an increase of the NC sample. This method was originally proposed by P.F. Loverre [75]. For the sake of simplicity, we assume to have a pure $\nu_{\mu}$ beam with a flux $\phi_{\mu}(E, x)$. The expected number of CC interactions in a detector of length $l$ located at a distance $L$ from the source can be written as

$$
n_{c c}(E)=\int_{L}^{L+l} \phi_{\mu}(E, x) \sigma_{c c}(E) d x
$$

Under the same assumptions the number of $\mathrm{NC}$ interactions is

$$
n_{n c}(E)=\int_{L}^{L+l} \phi_{\mu}(E, x) \sigma_{n c}(E) d x=\frac{\sigma_{n c}(E)}{\sigma_{c c}(E)} n_{c c}(E)
$$

In the absence of neutrino oscillations the total number of interactions is given by

$$
\begin{gathered}
N_{c c}=S \int n_{c c}(E) d E \\
N_{n c}=S \int n_{n c}(E) d E=\frac{\left\langle\sigma_{n c}\right\rangle}{\left\langle\sigma_{c c}\right\rangle} N_{c c}
\end{gathered}
$$

$S$ being a normalisation factor. If $\nu_{\mu} \leftrightarrow \nu_{\tau}$ neutrino oscillations occur, one also has to consider that the $\tau$ produced in $\nu_{\tau}$ CC interactions may decay into a muon. Therefore, if this muon is detected the $\nu_{\tau}$ event is classified as a $\nu_{\mu} \mathrm{CC}$ interaction. In addition, the cross-sections for $\nu_{\mu}$ and $\nu_{\tau}$ are slightly different $\left(\sigma_{\tau}<\sigma_{\mu}\right)$ and the interaction rate of $\nu_{\tau}$ is suppressed by a factor $\eta\left(=\sigma_{\tau} / \sigma_{\mu}\right)$. Taking into account $\nu_{\tau}$ interactions, the expected number of $\mathrm{CC}$ events in the presence of neutrino oscillations becomes 


$$
\begin{aligned}
N_{c c}^{o s c} & =S \int\left\{n_{c c}(E)\left[1-P\left(\nu_{\mu} \leftrightarrow \nu_{\tau}\right)\right]+n_{c c}(E) P\left(\nu_{\mu} \leftrightarrow \nu_{\tau}\right) \eta B R_{\tau \mu}\right\} d E \\
& =S\left\{\int n_{c c}(E) d E+\int n_{c c}(E)\left(\eta B R_{\tau \mu}-1\right) P\left(\nu_{\mu} \leftrightarrow \nu_{\tau}\right) d E\right\}
\end{aligned}
$$

while the number of $\mathrm{NC}$ interactions is

$$
N_{n c}^{o s c}=S \int\left\{\frac{\sigma_{n c}(E)}{\sigma_{c c}(E)} n_{c c}(E)+n_{c c}(E) P\left(\nu_{\mu} \leftrightarrow \nu_{\tau}\right) \eta\left(1-B R_{\tau \mu}\right)\right\} d E
$$

By using expressions (17), (18), (19) and (20) it is possible to derive the expected NC/CC ratio with or without oscillations

$$
\begin{gathered}
r_{n c / c c}=\frac{N_{n c}^{o s c}}{N_{c c}^{o s c}}=\frac{\int \frac{\sigma_{n c}(E)}{\sigma_{c c}(E)} n_{c c}(E) d E+\int n_{c c}(E) P\left(\nu_{\mu} \leftrightarrow \nu_{\tau}\right) \eta\left(1-B R_{\tau \mu}\right) d E}{\int n_{c c}(E) d E+\int n_{c c}(E) P\left(\nu_{\mu} \leftrightarrow \nu_{\tau}\right)\left(\eta B R_{\tau \mu}-1\right) d E} \\
R_{n c / c c}=\frac{N_{n c}}{N_{c c}}=\frac{\int \frac{\sigma_{n c}(E)}{\sigma_{c c}(E)} n_{c c}(E) d E}{\int n_{c c}(E) d E}
\end{gathered}
$$

If the difference $r_{n c / c c}-R_{n c / c c}$ is consistent with zero (no evidence for neutrino oscillations) a region in the $\Delta m^{2}-\sin ^{2} 2 \theta$ parameter space may be excluded at a given confidence level. Eq. (21) and (22) show that the difference $r_{n c / c c}-R_{n c / c c} \geq 0$. Hence, a one-sided Gaussian distribution can be used to define the $90 \%$ C.L. upper limit on the oscillation probability [1]. The difference $\left(r_{n c / c c}-R_{n c / c c}\right)$ is then

$$
\int\left\{n_{c c}\left[\left(1-1.28 \sigma_{R}\right) \eta\left(1-B R_{\tau \mu}\right)-R_{n c / c c}\left(\eta B R_{\tau \mu}-1\right)\right] P\left(\nu_{\mu} \leftrightarrow \nu_{\tau}\right)\right\} d E \leq 1.28 \sigma_{R} N_{c c}
$$

In the limit $\Delta m^{2} \gg E / L$, Eq. (23) can be written as

$$
\left(\sin ^{2} 2 \theta\right)_{\min }=\frac{2 \times 1.28 \sigma_{R}}{\left[\left(1-1.28 \sigma_{R}\right) \eta\left(1-B R_{\tau \mu}\right)-R_{n c / c c}\left(\eta B R_{\tau \mu}-1\right)\right]}
$$

The asymptotic value of $\sin ^{2} 2 \theta$ only depends on $\sigma_{R}$, i.e. the statistical accuracy of the experiment in measuring $R_{n c / c c}$. Possible systematical errors must be included in $\sigma_{R}$.

For $\sin ^{2} 2 \theta=1$ the limit on $\Delta m^{2}$ can be written as

$$
\left(\Delta m^{2}\right)_{\min }=\sqrt{\frac{\left(\sin ^{2} 2 \theta\right)_{\min }}{2 \times 1.267\left\langle\left(\frac{L}{E}\right)^{2}\right\rangle}}
$$




\subsubsection{Indirect appearance experiments searching for $\nu_{\mu} \leftrightarrow \nu_{e}$ oscillations}

We consider a $\nu_{\mu}$ beam with a contamination of $\nu_{e}$ equal to $f_{e}(E)$ such that $\phi_{e}(E)=f_{e}(E) \phi_{\mu}(E)$. If neutrino oscillations do not occur, because of the equality of the cross-sections the expected number of $\nu_{\mu}$ interactions at a given energy $E$ is $N_{\mu}(E)$ and the number of $\nu_{e}$ interactions is $N_{e}(E)=f_{e}(E) N_{\mu}(E)$. Conversely, if oscillations occur (for instance between $\nu_{\mu}$ and $\nu_{e}$ ) the observed number of events, corrected for detector acceptance and detection efficiency, is

$$
\begin{aligned}
& N_{e}^{o b s}(E)=P\left(\nu_{\mu} \leftrightarrow \nu_{e}\right) N_{\mu}(E)+\left(1-P\left(\nu_{\mu} \leftrightarrow \nu_{e}\right)\right) f_{e}(E) N_{\mu}(E) \\
& N_{\mu}^{o b s}(E)=\left(1-P\left(\nu_{\mu} \leftrightarrow \nu_{e}\right)\right) N_{\mu}(E)+P\left(\nu_{\mu} \leftrightarrow \nu_{e}\right) f_{e}(E) N_{\mu}(E)
\end{aligned}
$$

The total number of events is obtained by integrating expressions (26) and (27) over the neutrino spectrum

$$
\begin{aligned}
N_{e}^{o b s} & =\int\left\{P\left(\nu_{\mu} \leftrightarrow \nu_{e}\right) N_{\mu}(E)+\left(1-P\left(\nu_{\mu} \leftrightarrow \nu_{e}\right)\right) f_{e}(E) N_{\mu}(E)\right\} d E \\
& =\left\langle P\left(\nu_{\mu} \leftrightarrow \nu_{e}\right)\right\rangle\left\langle N_{\mu}(E)\right\rangle+\left(1-\left\langle P\left(\nu_{\mu} \leftrightarrow \nu_{e}\right)\right\rangle\right)\left\langle f_{e}(E)\right\rangle\left\langle N_{\mu}(E)\right\rangle \\
N_{\mu}^{o b s} & =\int\left\{\left(1-P\left(\nu_{\mu} \leftrightarrow \nu_{e}\right)\right) N_{\mu}(E)+P\left(\nu_{\mu} \leftrightarrow \nu_{e}\right) f_{e}(E) N_{\mu}(E)\right\} d E \\
& =\left(1-\left\langle P\left(\nu_{\mu} \leftrightarrow \nu_{e}\right)\right\rangle\right)\left\langle N_{\mu}(E)\right\rangle+\left\langle P\left(\nu_{\mu} \leftrightarrow \nu_{e}\right)\right\rangle\left\langle f_{e}(E)\right\rangle\left\langle N_{\mu}(E)\right\rangle
\end{aligned}
$$

A variable sensitive to neutrino oscillations is

$$
\frac{N_{e}^{o b s}-N_{e}}{N_{\mu}^{o b s}}=\frac{\left\langle P\left(\nu_{\mu} \leftrightarrow \nu_{e}\right)\right\rangle\left(1-\left\langle f_{e}(E)\right\rangle\right)}{1-\left(1-\left\langle f_{e}(E)\right\rangle\right)\left\langle P\left(\nu_{\mu} \leftrightarrow \nu_{e}\right)\right\rangle}
$$

where $N_{e}$ is the number of $\nu_{e}$ events detected in the absence of oscillations. The expression (32) is independent of the absolute flux $\phi_{\mu}(E)$.

If the difference $\left(N_{e}^{o b s}-N_{e}\right) \equiv N$ is consistent with zero (and the statistics large enough) it can be assumed distributed as a Gaussian with a $90 \%$ C.L upper limit on the signal equal to $N+1.28 \sigma$. The excluded region in the $\Delta m^{2}-\sin ^{2} 2 \theta$ plane can then be obtained by solving the equation

$$
\frac{N+1.28 \sigma}{N_{\mu}^{o b s}} \geq \frac{\left\langle P\left(\nu_{\mu} \leftrightarrow \nu_{e}\right)\right\rangle\left(1-\left\langle f_{e}(E)\right\rangle\right)}{1-\left(1-\left\langle f_{e}(E)\right\rangle\right)\left\langle P\left(\nu_{\mu} \leftrightarrow \nu_{e}\right)\right\rangle}
$$

For large $\Delta m^{2}$ values Eq. (33) assumes the asymptotic form

$$
\left(\sin ^{2} 2 \theta\right)_{\min }=\frac{2(N+1.28 \sigma)}{(N+1.28 \sigma)\left(1-f_{e}\right)+\left(1-f_{e}\right) N_{\mu}^{o b s}}
$$


If $N_{e}^{o b s}$ is consistent with $N_{e}$ then $\sigma=\sqrt{N_{e}}$ and, therefore,

$$
\left(\sin ^{2} 2 \theta\right)_{\min }=\frac{2 \times 1.28 \sigma}{1.28 \sigma\left(1-f_{e}\right)+\left(1-f_{e}\right) N_{\mu}^{o b s}}
$$

For $\sin ^{2} 2 \theta=1$ the mean value of the oscillation probability can be written as

$$
\left\langle P\left(\nu_{\mu} \leftrightarrow \nu_{e}\right)\right\rangle=\left(\Delta m^{2}\right)^{2}\left\langle\left(1.27 \frac{L}{E}\right)^{2}\right\rangle
$$

so that

$$
\begin{gathered}
\frac{N+1.28 \sigma}{N_{\mu}^{o b s}} \geq \frac{\left(\Delta m^{2}\right)^{2}\left\langle\left(1.27 \frac{L}{E}\right)^{2}\right\rangle\left(1-\left\langle f_{e}(E)\right\rangle\right)}{1-\left(1-\left\langle f_{e}(E)\right\rangle\right)\left(\Delta m^{2}\right)^{2}\left\langle\left(1.27 \frac{L}{E}\right)^{2}\right\rangle} \\
\frac{1.28 \sigma}{N_{\mu}^{o b s}} \geq \frac{\left(\Delta m^{2}\right)^{2}\left\langle\left(1.27 \frac{L}{E}\right)^{2}\right\rangle\left(1-\left\langle f_{e}(E)\right\rangle\right)}{1-\left(1-\left\langle f_{e}(E)\right\rangle\right)\left(\Delta m^{2}\right)^{2}\left\langle\left(1.27 \frac{L}{E}\right)^{2}\right\rangle}
\end{gathered}
$$

\subsubsection{Direct appearance experiments searching for $\nu_{\mu} \leftrightarrow \nu_{\tau}$ oscillations}

One assumes to deal with a $\nu_{\mu}$ beam with negligible $\nu_{\tau}$ contamination. The ratio of the observed numbers of $\nu_{\tau} \mathrm{CC}$ over $\nu_{\mu} \mathrm{CC}$ interactions can be expressed as

$$
\begin{aligned}
\frac{N_{\tau}}{N_{\mu}} & =\frac{\int \phi_{\mu}(E) \sigma_{\tau} P\left(\nu_{\mu} \leftrightarrow \nu_{\tau}\right) \varepsilon_{\tau} d E B R}{\int \phi_{\mu}(E) \sigma_{\mu} \varepsilon_{\mu} d E} \\
& =\frac{\int \phi_{\mu}(E) \sigma_{\tau} P\left(\nu_{\mu} \leftrightarrow \nu_{\tau}\right) \varepsilon_{\tau} B R d E}{\int \phi_{\mu}(E) P\left(\nu_{\mu} \leftrightarrow \nu_{\tau}\right) \sigma_{\tau} d E} \frac{\int \phi_{\mu}(E) \sigma_{\mu} d E}{\int \phi_{\mu}(E) \sigma_{\mu} \varepsilon_{\mu} d E} \frac{\int \phi_{\mu}(E) P\left(\nu_{\mu} \leftrightarrow \nu_{\tau}\right) \sigma_{\tau} d E}{\int \phi_{\mu}(E) \sigma_{\mu} d E} \\
& =B R \frac{\left\langle\varepsilon_{\tau}\right\rangle}{\left\langle\varepsilon_{\mu}\right\rangle} \frac{\int \phi_{\mu}(E) P\left(\nu_{\mu} \leftrightarrow \nu_{\tau}\right) \sigma_{\tau} d E}{\int \phi_{\mu}(E) P\left(\nu_{\mu} \leftrightarrow \nu_{\tau}\right) d E} \frac{\int \phi_{\mu}(E) d E}{\int \phi_{\mu}(E) \sigma_{\mu} d E} \frac{\int \phi_{\mu}(E) P\left(\nu_{\mu} \leftrightarrow \nu_{\tau}\right) d E}{\int \phi_{\mu}(E) d E} \\
& =B R \frac{\left\langle\varepsilon_{\tau}\right\rangle}{\left\langle\varepsilon_{\mu}\right\rangle} \frac{\left\langle\sigma_{\tau}\right\rangle}{\left\langle\sigma_{\mu}\right\rangle}\left\langle P\left(\nu_{\mu} \leftrightarrow \nu_{\tau}\right)\right\rangle
\end{aligned}
$$

where $\varepsilon$ is the $\tau$ detection efficiency and $B R$ the total branching ratio of the $\tau$ decay channels to which the experiment is sensitive.

The sensitivity to oscillations can be determined by evaluating the mean values in Eq. (34) as a function of $\Delta m^{2}$ and $\sin ^{2} 2 \theta .\left\langle\varepsilon_{\tau}\right\rangle$ and $\left\langle\sigma_{\tau}\right\rangle$ include the convolution of the $\nu_{\mu}$ spectrum with the oscillation probability. $\left\langle P\left(\nu_{\mu} \leftrightarrow \nu_{\tau}\right)\right\rangle$ only depends on the $\nu_{\mu}$ spectrum and in the standard two-flavour approximation can be written as

$$
\left\langle P\left(\nu_{\mu} \leftrightarrow \nu_{\tau}\right)\right\rangle=\sin ^{2} 2 \theta\left\langle\sin ^{2} \frac{1.267 \Delta m^{2} L}{E}\right\rangle
$$


If the experiment observes no events with negligible expected background, the $90 \%$ C.L. upper limit on the signal amounts to 2.44 events following the prescriptions of the frequentist statistical approach [76]. For large $\Delta m^{2}$ values Eq. (34) takes the asymptotic form

$$
\left(\sin ^{2} 2 \theta\right)_{\min }=\frac{2 \times 2.44\left\langle\varepsilon_{\mu}\right\rangle\left\langle\sigma_{\mu}\right\rangle}{N_{\mu}\left\langle\varepsilon_{\tau}\right\rangle\left\langle\sigma_{\tau}\right\rangle B R}
$$

From Eq. (35) one deduces that in the total absence of background the sensitivity of a direct appearance experiment linearly improves with the number of detected $\nu_{\mu} \mathrm{CC}$ events. The asymptotic limit on $\Delta m^{2}$ has an expression analogous to Eq. (25).

\subsubsection{Short baseline versus long baseline appearance experiments}

As already discussed in Section 1 and shown in Table 1 experiments searching for neutrino oscillations with accelerator beams can be classified as short or long baseline according to the distance $L$ of the detector from the neutrino source, relatively to the beam energy $E$.

Let us now consider, for example, a background-free experiment searching for $\nu_{\mu} \leftrightarrow \nu_{\tau}$ oscillations. In the case of a negative search (Eq. (35)), the minimum $\sin ^{2} 2 \theta$ to which the experiment is sensitive is proportional to the inverse of the number of detected $\nu_{\mu}$ events $\left(N_{\mu}\right)$, in turn proportional to $L^{-2}$ and to the detector mass $M_{d}$

$$
N_{\mu}=N_{a} M_{d} \int \phi_{\mu}(E) \sigma_{\mu}(E) \varepsilon_{\mu}(E) d E \simeq \frac{N_{a} M_{d}}{L^{2}} \int f(E) \sigma_{\mu}(E) \varepsilon_{\mu}(E) d E
$$

where $f(E)$ is a function which takes into account the energy dependence of the neutrino flux and $N_{a}$ is the Avogadro number. Using Eqs. (25), (35) and (36) the sensitivity to $\Delta m^{2}$ is

$$
\left(\Delta m^{2}\right)_{\min }=\sqrt{\frac{2.44\left\langle\varepsilon_{\mu}\right\rangle\left\langle\sigma_{\mu}\right\rangle}{\int N_{a} M_{d} f(E) \sigma_{\mu}(E) \varepsilon_{\mu}(E) d E} \times \frac{1}{\left\langle\varepsilon_{\tau}\right\rangle\left\langle\sigma_{\tau}\right\rangle B R} \times \frac{E^{2}}{1.267}}
$$

Therefore, the sensitivity of a background-free appearance experiment to $\Delta m^{2}$ depends on the neutrino beam energy, on the number of detected events and not on the baseline $L$. The reason is that the increase of the oscillation probability with $L^{2}$ is compensated by the reduction in flux $\phi_{\mu}(E)$, which goes like $L^{-2}$. The sensitivity improves with the square-root of the detector mass: $\left(\Delta m^{2}\right)_{\min } \propto 1 / \sqrt{M_{d}}$.

In the presence of background the scenario is slightly modified. For example, we consider the $\nu_{\mu} \leftrightarrow \nu_{e}$ indirect appearance search discussed in Section 3.2.2. In this case an irreducible background is given by the intrinsic contamination of electron neutrinos in the beam. $\left(\sin ^{2} 2 \theta\right)_{\min }$ is proportional to the square-root of the expected background and is proportional to the inverse of the observed number of events (assuming $\left.N_{\mu} \gg \sqrt{N_{e}}\right)$. Therefore, being that both $N_{\mu}$ and $N_{e}$ are proportional to $L^{-2}$ and $M_{d},\left(\sin ^{2} 2 \theta\right)_{\text {min }}$ becomes proportional to $L / \sqrt{M_{d}}$. This means that the sensitivity to $\sin ^{2} 2 \theta$ improves linearly with decreasing the distance source-detector. On the other hand, it can be shown that the sensitivity to $\Delta m^{2}$ improves with the square-root of $L$ and with the fourth-root of $M_{d}$. 


\begin{tabular}{||c|c|c||}
\hline & No background & Background \\
\hline \hline$\left(\sin ^{2} 2 \theta\right)_{\min }$ & $\propto L^{2} ; \propto 1 / M_{d}$ & $\propto L ; \propto 1 / \sqrt{M_{d}}$ \\
\hline$\left(\Delta m^{2}\right)_{\min }$ & No $L$ dependence; $\propto 1 / \sqrt{M_{d}}$ & $\propto 1 / \sqrt{L} ; \propto 1 / \sqrt[4]{M_{d}}$ \\
\hline \hline
\end{tabular}

Table 2: Dependence of the oscillation parameter on experimental quantities.

An interesting case occurs when the expected background is very small $(\mathcal{O}(1)$ event). In this condition, the Poisson statistics has to be considered to evaluate the upper limit on the number of signal events. This limit is no longer proportional to the square-root of the expected number of background events. By using the Feldman and Cousins approach [76], it can be shown that with an expected background of 0.1 events and an increase in statistics of a factor 10, the upper limit (sensitivity to $\sin ^{2} 2 \theta$ ) increases (decreases) only by about $30 \%$.

In Table 2 the dependence of $\left(\Delta m^{2}\right)_{\text {min }}$ and $\left(\sin ^{2} 2 \theta\right)_{\min }$ as a function of $L$ and $M_{d}$ are shown. From the above discussion it follows that the relevant quantity to be varied in order to explore different oscillation parameter regions is the baseline $L$. Large mass detector targets are needed in practice to reach small values of $\left(\Delta m^{2}\right)_{\min }$. However, the dependence of the experimental sensitivity on the detector mass is weak.

In the ideal case of a background-free experiment, the detector (with the highest possible mass) should be located as close as possible to the neutrino source in order to increase the number of observed events and hence the sensitivity to $\sin ^{2} 2 \theta$. The sensitivity to $\Delta m^{2}$ is independent of $L$ and one could benefit from a large detector mass. Unfortunately, in real life the total absence of background is very difficult to achieve.

\section{Solar neutrinos}

\subsection{Experimental results}

The Sun is a very effective neutrino producer as is any other star in the Universe. Electron neutrinos come from the thermonuclear reactions occurring inside the Sun mostly through the chain indicated in Fig. 7. Every second, a flux of about 60 billion solar neutrinos traverses each square centimeter of the Earth's surface. Detectors on the Earth can measure this flux and compare it with the expectations from theoretical models. Each reaction is characterised by a specific energy spectrum of the emitted neutrinos. The spectra can be predicted by theory and are graphically shown in Fig. 8.

The first experimental evidence consistent with the hypothesis of neutrino oscillations is about thirty years old. It originated from a discrepancy between the measured detection rate in the solar neutrino experiment by R. Davis [77] and the theoretical calculations [78]: a statistically significant deficit in the measured flux of $\nu_{e}$ was observed. This effect is commonly referred to as the solar neutrino problem. The deficit can be interpreted assuming that electron neutrinos oscillate into a different flavour in their travel towards the Earth. So far, five different experiments confirmed and consolidated the measurement of the neutrino deficit.

Although this review focuses on experiments exploiting accelerator neutrino beams, we briefly summarise the current experimental scenario and our understanding of the solar neutrino problem. For an example of exhaustive review we refer to [14].

The first solar neutrino experiment was the radiochemical chlorine experiment, which has been running 


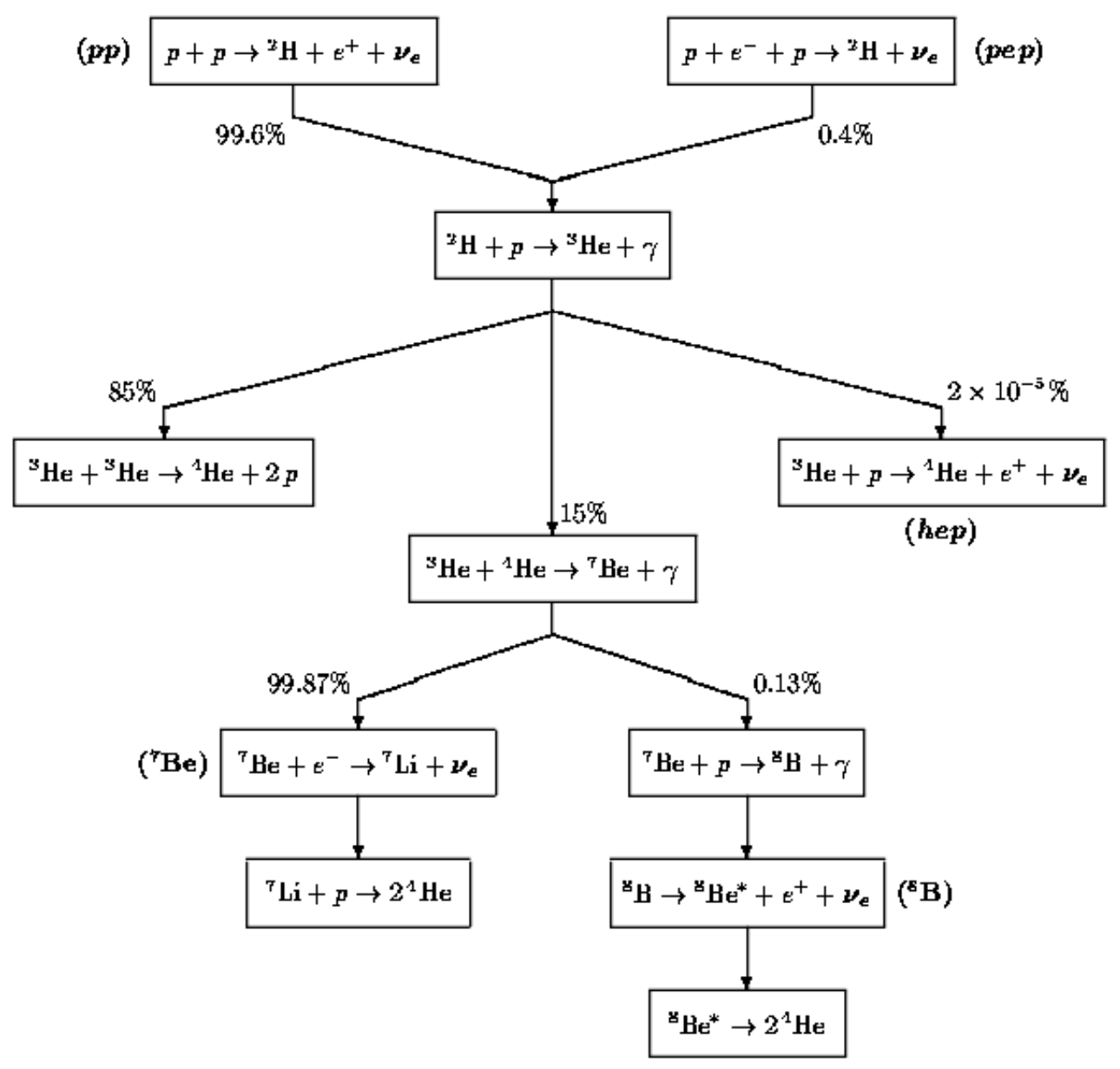

Figure 7: Solar reactions proceeding through the main $p p$ chain [65]. 


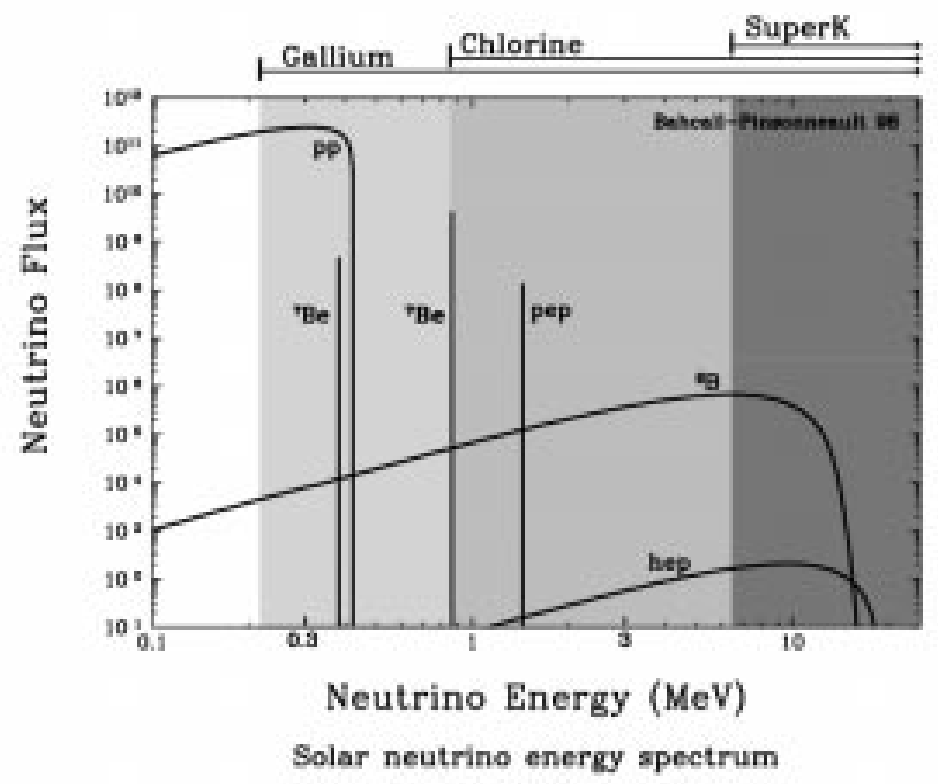

Figure 8: Predicted spectrum of solar neutrinos [11]. Neutrino fluxes from continuum sources (such as $p p$ and ${ }^{8} \mathrm{~B}$ ) are given in $\mathrm{cm}^{-2} \mathrm{~s}^{-1} \mathrm{MeV}^{-1}$. Those from discrete sources (such as ${ }^{7} \mathrm{Be}$ ) are given in $\mathrm{cm}^{-2} \mathrm{~s}^{-1}$. Also shown are the thresholds for the different reactions used for neutrino detection.

in the Homestake Gold mine at Lead in South Dakota for about 30 years. The apparatus consists of 615 ton of $C_{2} C l_{4}$ (perchloroethylene) and allows the detection of solar neutrinos $\left(\nu_{e}\right)$ through the inverse $\beta$-decay reaction

$$
\nu_{e}+{ }_{17}^{37} \mathrm{Cl} \rightarrow{ }_{18}^{37} \mathrm{Ar}+e^{-}
$$

The energy threshold for this process is $E_{\nu}=0.813 \mathrm{MeV}$, just below the $0.862 \mathrm{MeV}{ }^{7} \mathrm{Be}$ line. Therefore, the detected neutrino flux is mainly due to ${ }^{7} B e$ and ${ }^{8} B$ reactions.

The lifetime of ${ }_{18}^{37} A r$ is about 34 days. The experimental method exploited for its detection is based on the electron capture reaction

$$
{ }_{18}^{37} \mathrm{Ar}+e^{-} \rightarrow{ }_{17}^{37} \mathrm{Cl}+\nu_{e}+X_{\text {rays }}
$$

The most recent results refer to data taken between 1970 and 1994. The measured capture rate amounts to $2.56 \pm 0.16 \pm 0.16 S N U$ [79]. The $S N U$ (Solar Neutrino Unit) is a convenient unit to measure the rates of solar neutrino experiments. It corresponds to $10^{-36}$ interactions per target atom per second.

The predicted solar neutrino flux comes from different reactions which occur inside the Sun [11]. Their contribution in terms of $S N U_{\mathrm{s}}$ is

$$
7.7_{-1.0}^{+1.2} S N U=0.0(p p)+0.2(p e p)+1.15\left({ }^{7} B e\right)+5.9\left({ }^{8} B\right)+0.5(C N O)
$$

The disagreement between the predicted and the measured flux is statistically significant. It corresponds to more than $3.5 \sigma$. 
Another category of radiochemical solar neutrino experiments makes use of gallium instead of chlorine, in order to profit from the lower energy threshold $(0.233 \mathrm{MeV})$ of the reaction

$$
\nu_{e}+{ }_{31}^{71} G a \rightarrow{ }_{32}^{71} G e+e^{-}
$$

These experiments are therefore sensitive to the $p p$ component of the solar neutrino spectrum. The luminosity of the Sun is mainly determined by the $p p$ chain and is a well measured quantity. This constraint affects positively the prediction of the solar neutrino flux coming from the $p p$ reaction.

Two Collaborations performed this experiment: GALLEX [80] and SAGE [81]. The first detector is located underground in the Gran Sasso Laboratory and uses 30.7 ton of gallium containing 12 ton of ${ }^{71} \mathrm{Ga}$. The second one is placed in a laboratory under Mount Andryrchi in the Baksan Valley and consists of 60 ton of natural gallium. The ${ }_{32}^{71} G e$ atoms have a lifetime of about 11 days and are detected through the capture reaction

$$
{ }_{32}^{71} G e+e^{-} \rightarrow{ }_{31}^{71} G a+\nu_{e}+X_{\text {rays }}
$$

The predicted solar neutrino flux is [11]

$$
129_{-6}^{+8} S N U=69.6(p p)+2.8(p e p)+34.4\left({ }^{7} B e\right)+12.4\left({ }^{8} B\right)+9.8(C N O)
$$

and it has to be compared with the rates measured by GALLEX [80] and SAGE [81], respectively of

$$
77.5 \pm 6.2_{-4.7}^{+4.3} S N U, \quad 67.2_{-7.0-3.0}^{+7.2+3.5} S N U
$$

Also in this case the disagreement between predicted and measured flux is at the level of $3.5 \sigma$.

The capability of GALLEX and SAGE in counting ${ }_{32}^{71} \mathrm{Ge}$ atoms has been established by using a ${ }^{51} \mathrm{Cr}$ radioactive source for calibration. The source has a lifetime of 27.7 days. Emitted neutrinos are monoenergetic with energies of $0.746 \mathrm{MeV}(81 \%), 0.751 \mathrm{MeV}(9 \%), 0.426 \mathrm{MeV}$ (9\%) and $0.431 \mathrm{MeV}$ (1\%). The two groups measured the flux ratio $\mathrm{R}$ (measured/expected) by knowing (with an accuracy of $\sim 1 \%$ ) the absolute neutrino intensity of the source. GALLEX performed the calibration twice and obtained $1.01_{-0.11}^{+0.12}$ and $0.84_{-0.11}^{+0.12}$ with the combined result of $0.93 \pm 0.08$ [82]. The SAGE Collaboration obtained $0.95 \pm 0.12_{-0.027}^{+0.035}$ [83]. Both measurements are consistent with the expectations from the source intensity proving the reliability of the technique used to detect ${ }_{32}^{71} G e$ decays.

The GALLEX Collaboration also made a study of the efficiency in detecting ${ }^{71} G e$ atoms by using radioactive ${ }^{71} \mathrm{As}$ atoms [84]. ${ }^{71} \mathrm{As}$ decays through electron capture and positron emission to ${ }^{71} \mathrm{Ge}$ with kinematics which mimics the solar neutrino capture. This experiment showed that the recovery of ${ }^{71} G e$ is $100 \%$ with only $1 \%$ uncertainty [84].

Table 3 summarises the expected solar neutrino fluxes from the different reactions together with the same quantities as predicted for Chlorine and Gallium experiments.

Another technique exploited for the detection of solar neutrinos is the one originally adopted by the Kamiokande experiment [85]. The experiment is based on the use of a large water Cerenkov detector located $1000 \mathrm{~m}$ underground in the Kamioka mine in Japan. The detector is sensitive to both solar and atmospheric neutrinos (Section 5). A cylindrical tank contains $4.5 \mathrm{kton}$ of water. The inner volume of 2140 ton of water 


\begin{tabular}{||c|c|c|c||}
\hline Source & Flux on Earth $\left(10^{10} \mathrm{~cm}^{-2} \mathrm{~s}^{-1}\right)$ & Cl (SNU) & Ga (SNU) \\
\hline \hline$p p$ & $5.94 \pm 0.01$ & 0.0 & 69.6 \\
\hline$p e p$ & $0.0139 \pm 0.0001$ & 0.2 & 2.8 \\
\hline$h e p$ & $2.10 \times 10^{-7}$ & 0.0 & 0.0 \\
\hline${ }^{7} \mathrm{Be}$ & $0.480 \pm 0.009$ & 1.15 & 34.4 \\
\hline${ }^{8} \mathrm{~B}$ & $\left(5.15_{-0.14}^{+0.19}\right) \times 10^{-4}$ & 5.9 & 12.4 \\
\hline${ }^{13} \mathrm{~N}$ & $\left(6.05_{-0.13}^{+0.19}\right) \times 10^{-2}$ & 0.1 & 3.7 \\
\hline${ }^{15} \mathrm{O}$ & $\left(5.32_{-0.15}^{+0.22}\right) \times 10^{-2}$ & 0.4 & 6.0 \\
\hline${ }^{17} \mathrm{~F}$ & $\left(6.33_{-0.11}^{+0.12}\right) \times 10^{-4}$ & 0.0 & 0.1 \\
\hline \hline
\end{tabular}

Table 3: Neutrino fluxes and related theoretical uncertainties from the Standard Solar Model [11] for Chlorine and Gallium experiments.

is viewed by 948 photo-multiplier tubes (PMT) allowing to give images of the Cerenkov rings. For the solar neutrino analysis the innermost part of the detector is used corresponding to a fiducial mass of 680 ton of water.

Kamiokande identifies the process $\nu+e^{-} \rightarrow \nu+e^{-}$by detecting the Cerenkov light of the scattered electron. For this reaction there is in principle no kinematical threshold. However, due to the need of background rejection (Cerenkov light from radioactivity and cosmic-ray particles) a threshold of $\sim 5 \mathrm{MeV}$ is required making the experiment only sensitive to ${ }^{8} B$ neutrinos.

The method used by Kamiokande has the following advantages with respect to radiochemical experiments

- it is a real time detection, thus sensitive to short term variations of the solar neutrino flux (day-night effect and seasonal variations, discussed later);

- it provides correlation between the electron and neutrino direction, if the energy of the scattered electron is appreciably higher than the electron mass;

- spectral information can be derived.

The neutrino signal is extracted by taking into account the directional correlation with the Sun and considering events with energy above threshold $(7 \mathrm{MeV})$ and below $20 \mathrm{MeV}$. Due to the lower energy cut the relation $E_{e} \gg m_{e}$ holds. This allows a good angular correlation. For example, $26 \%$ of the electrons with $10 \mathrm{MeV}$ have the same direction of the incoming neutrinos.

The energy calibration of the Kamiokande detector has been performed by using gamma rays from a nickel source. The predicted solar neutrino flux [11] amounts to

$$
5.15_{-0.7}^{+1.0} \times 10^{6} \mathrm{~cm}^{-2} \mathrm{~s}^{-1}
$$

The latter has to be compared with the measured rate [85] of

$$
(2.80 \pm 0.19 \pm 0.33) \times 10^{6} \mathrm{~cm}^{-2} \mathrm{~s}^{-1}
$$

The Kamiokande experiment was completed in 1995. In April 1996 the 50 kton follow-up SuperKamiokande detector became operational. It is conceptually similar to its predecessor and is described in 


\begin{tabular}{||c|c|c|c||}
\hline Experiment & Result & Theory & Result/Theory \\
\hline \hline Homestake & $2.56 \pm 0.16 \pm 0.16$ & $7.7_{-1.0}^{+1.2}$ & $0.33 \pm 0.03$ \\
\hline GALLEX & $77.5 \pm 6.2_{-4.7}^{+4.3}$ & $129_{-6}^{+8}$ & $0.60 \pm 0.06$ \\
\hline SAGE & $67.2_{-7.0-3.0}^{+7.2+3.5}$ & $129_{-6}^{+8}$ & $0.52 \pm 0.06$ \\
\hline Kamiokande & $2.80 \pm 0.19 \pm 0.33$ & $5.15_{-0.7}^{+1.0}$ & $0.54 \pm 0.07$ \\
\hline Super-Kamiokande & $2.45 \pm 0.04 \pm 0.07$ & $5.15_{-0.7}^{+1.0}$ & $0.475_{-0.008}^{+0.008} \pm 0.013$ \\
\hline \hline
\end{tabular}

Table 4: Results from solar neutrino experiment are compared with theoretical predictions. The quantities are $S N U$, except for Kamiokande and Super-Kamiokande $\left(10^{6} \mathrm{~cm}^{-2} \mathrm{~s}^{-1}\right)$.

Section 13.1. Results from 825 days of data taking are now available. For that analysis a fiducial volume of 22.5 kton and an energy threshold of $\sim 5 \mathrm{MeV}$ are used.

The measured flux is [86]

$$
(2.45 \pm 0.04 \pm 0.07) \times 10^{6} \mathrm{~cm}^{-2} \mathrm{~s}^{-1}
$$

The central value is slightly smaller than the one obtained by Kamiokande. This discrepancy can be due to a difference in the energy calibrations. In order to settle this issue the Super-Kamiokande Collaboration exploited electrons from a dedicated LINAC accelerator. Electrons with energies within the range 5 $16 \mathrm{MeV}$ and hitting the detector fiducial volume at different positions are used. This allowed to determine the absolute energy scale with a 1\% accuracy. For details about the results of this study we refer to [87].

The results of solar neutrino experiments are summarised in Table 4 together with the theoretical predictions. Super-Kamiokande also reported on the evaluation of a possible day-night effect, which is expected if neutrino oscillations are the explanation of the solar neutrino deficit. Neutrinos oscillated in the Sun $\left(\nu_{\mu}\right.$ or $\nu_{\tau}$ ) could re-convert inside the Earth into the original $\nu_{e}$ flavour (see next Section). This effect would lead to a higher rate of detected $\nu_{e}$ during the night. The measured day-night asymmetry is [86]

$$
\frac{N-D}{D+N}=0.065 \pm 0.031 \pm 0.013
$$

where $D$ and $N$ stand for day-time and night-time event rates, respectively. A signal of $1.9 \sigma$ is found: still not enough to confirm the effect.

The distortion of the neutrino energy spectrum is another expected feature possibly due to neutrino oscillations. The shape of the spectrum determines the energy distribution of the recoil electron produced in the $\nu_{e} e$ interaction. Departure from the predictions of the electroweak theory would be indication of new physics.

Super-Kamiokande results on the recoil energy spectrum based on 825 days of data taking are reported in [86]. The fit to the data obtained assuming the undistorted spectrum is only marginally acceptable. Unfortunately, the statistical and systematic errors are still too large to draw firm conclusions. An explanation of the effect in terms of a different than expected flux of hep neutrinos would require a dramatic increase of this flux by a factor thirty [86]. 


\subsection{The solar neutrino problem}

The measurements of the solar neutrino flux reported in the previous Section clearly show a disagreement with the theoretical predictions. A thorough discussion on the subject, which is beyond the objectives of this review, can be found in [14]. Apart from explanations involving alternative solar models [14] two possible solutions which contemplate neutrino oscillations may be envisaged. The first one calls for neutrino oscillations in vacuum. Due to the large Sun-Earth distance and to the low energy of solar neutrinos, oscillations with $\Delta m^{2} \sim 10^{-11} \mathrm{eV}^{2}$ have to be advocated. The second one solves the solar neutrino problem by invoking the MSW effect $[12,13]$.

The latter effect explains the solar neutrino deficit as a result of the $\nu_{e}$ conversion into $\nu_{\mu}$ or $\nu_{\tau}$ in the dense solar matter. It is basically due to the fact that $\nu_{e}$, unlike $\nu_{\mu}$ and $\nu_{\tau}$, can undergo both $\mathrm{NC}$ and CC interactions with the electrons of the solar matter. In the oscillation of $\nu_{e}$ into another flavour an additional term is introduced in the matrix describing the flavour evolution. For a complete discussion on the subject we refer to $[88,89]$.

The solar neutrino problem is thirty years old and several experiments have been investigating it. Recent developments in the field of helioseismology [11] indicate that the Standard Solar Model is correct so disfavouring astrophysical solutions. However, due to uncertainties in the absolute neutrino flux there is still no decisive argument in favour of solar neutrino oscillations. Therefore, flux independent measurements (the so-called smoking-guns) are awaited in order to have evidence independent of the SSM predictions. Possible smoking-guns are the solar neutrino energy spectrum distortion, the day-night effect and the seasonal variations (the distance Sun-Earth changes during the Earth revolution).

The energy spectrum distortion is common to both vacuum oscillations and to the MSW effect. On the other hand, day-night asymmetry and seasonal variation are peculiar to MSW and to vacuum oscillation solutions, respectively. As discussed in the previous Section Super-Kamiokande data indicate a $\sim 2 \sigma$ effect for the day-night asymmetry and a possible spectrum distortion. No evidence has been reported for seasonal variations.

A combined fit of all available solar neutrino data obtained following the prescription given in [14] gives four possible allowed regions in the $\Delta m^{2}-\sin ^{2} 2 \theta$ plane. The vacuum oscillation solution constraints $\Delta m^{2} \sim 8 \times 10^{-11} e V^{2}$ and $\sin ^{2} 2 \theta>0.7$ (Fig. 9). Three possible MSW solutions are also allowed: the LMA (Large Mixing Angle) solution $\left(\Delta m^{2} \sim 10^{-5} \mathrm{eV}^{2}, \sin ^{2} 2 \theta>0.7\right.$ ), the SMA (Small Mixing Angle) solution $\left(\Delta m^{2} \sim 10^{-5} \mathrm{eV}^{2}, \sin ^{2} 2 \theta \sim 10^{-3}\right)$ and the LOW (low probability, low mass) solution $\left(\Delta m^{2} \sim 10^{-7} \mathrm{eV}^{2}, \sin ^{2} 2 \theta \sim 1\right)$ (Fig. 10). The present statistics is not sufficient to discriminate among them.

\section{$5 \quad$ Atmospheric neutrinos}

\subsection{The atmospheric neutrino anomaly}

Atmospheric neutrinos are produced by primary cosmic rays $(\mathrm{CR})$ interacting with the atmosphere and creating a large number of secondary $\pi$ and $K$ mesons, mainly with energies of a few $G e V$. Due to the low density of the atmosphere essentially all the mesons decay before interacting: $\pi / K \rightarrow \mu+\nu_{\mu}\left(\bar{\nu}_{\mu}\right)$. Most of the muons decay before reaching the ground: $\mu \rightarrow e+\nu_{e}\left(\bar{\nu}_{e}\right)+\bar{\nu}_{\mu}\left(\nu_{\mu}\right)$. If all muons decayed one would expect $N\left(\nu_{\mu}\right) / N\left(\nu_{e}\right)=2$ (ignoring the distinction between $\nu$ and $\bar{\nu}$ ) with $N\left(\nu_{\mu}\right)\left(N\left(\nu_{e}\right)\right.$ ) indicating the 


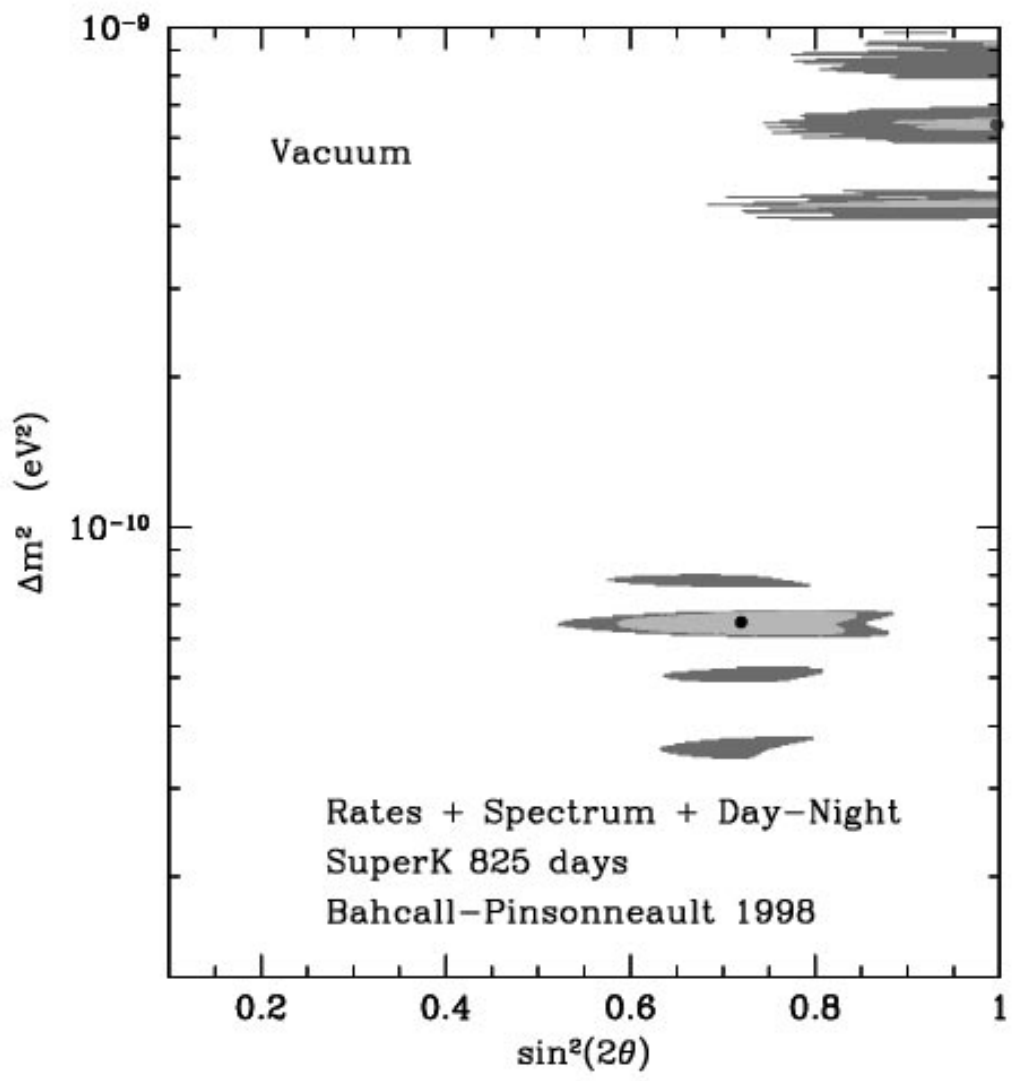

Figure 9: Parameters allowed by the vacuum oscillation solution of the solar neutrino problem. The black point shows the best fit value; light (dark) grey zones indicate the $90 \%$ (99\%) C.L. allowed region. 


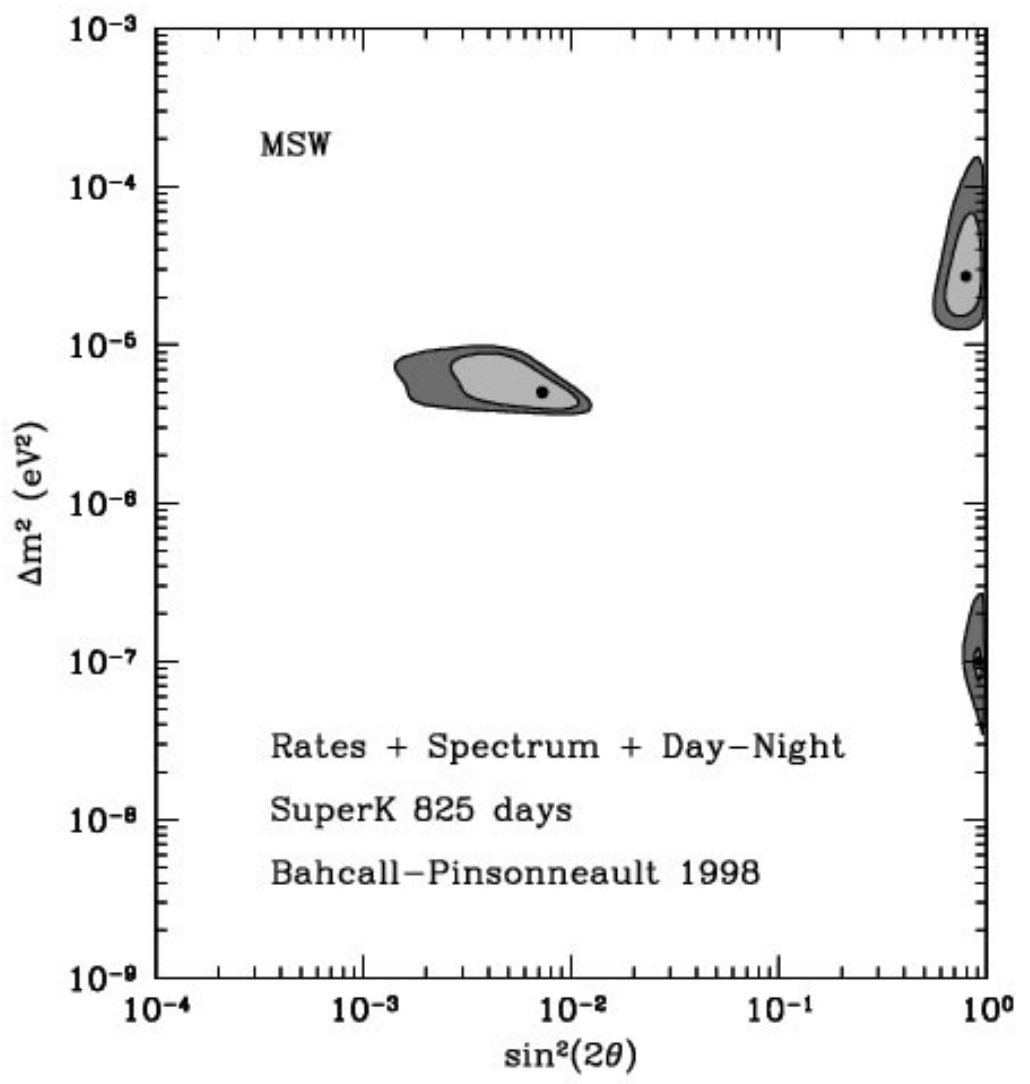

Figure 10: Parameters allowed by the MSW solution of the solar neutrino problem. The black point shows the best fit value; light (dark) grey zones indicates the $90 \%$ (99\%) C.L. allowed region. 


\begin{tabular}{||c|c|c||}
\hline Experiment & $R$ & Stat. significance (kton - year) \\
\hline \hline NUSEX & $0.99_{-0.25}^{+0.35}$ & 0.74 \\
\hline Frejus & $1.00 \pm 0.15 \pm 0.08$ & 2.0 \\
\hline IMB & $0.54 \pm 0.05 \pm 0.11$ & 7.7 \\
\hline Kamiokande (sub-GeV) & $0.60_{-0.05}^{+0.06} \pm 0.05$ & 7.7 \\
\hline Kamiokande (multi-GeV) & $0.57_{-0.07}^{+0.08} \pm 0.07$ & 7.7 \\
\hline Soudan2 & $0.68 \pm 0.11 \pm 0.06$ & 4.6 \\
\hline Super-Kamiokande (sub-GeV) & $0.680_{-0.022}^{+0.023} \pm 0.053$ & 52.26 \\
\hline Super-Kamiokande (multi-GeV) & $0.678_{-0.039}^{+0.042} \pm 0.080$ & 52.26 \\
\hline \hline
\end{tabular}

Table 5: Summary of the double ratio $R$ ratio measurements for atmospheric neutrinos.

number of events induced by muon (electron) neutrinos. Due to relativistic effects, a lower value of the ratio is expected at high energies.

For a given experiment sensitive to $\nu_{e}$ and $\nu_{\mu}$ with $N_{t}$ target particles and running over a time $t_{\text {exp }}$, the observed number of events of the flavour $l\left(e^{ \pm}\right.$or $\left.\mu^{ \pm}\right)$is

$$
\frac{d N_{l}\left(\theta_{z}, p_{l}\right)}{d \Omega_{\theta_{z}} d p_{l}}=t_{\exp } \sum_{\nu, \bar{\nu}} \int N_{t} \frac{d \phi_{\nu_{l}}^{ \pm}\left(E_{\nu}, \theta_{z}\right)}{d \Omega_{\theta_{z}} d E_{\nu}} \frac{\sigma^{ \pm}\left(E_{\nu}, p_{l}\right)}{d p_{l}} F\left(q^{2}\right) d E_{\nu}
$$

where $p_{l}$ is the lepton momentum, $E_{\nu}$ the neutrino energy, $\theta_{z}$ the neutrino zenith angle, $\phi_{\nu_{l}}^{ \pm}\left(E_{\nu}, \theta_{z}\right)$ the neutrino flux, $\sigma^{ \pm}\left(E_{\nu}, p_{l}\right)$ the total cross-section and $F\left(q^{2}\right)$ a function accounting for nuclear effects. Several authors [90,91] have calculated $\phi_{\nu_{l}}^{ \pm}\left(E_{\nu}, \theta_{z}\right)$ and the results agree within $10 \%$ in the range $200 \mathrm{MeV}<$ $E_{\nu}<200 \mathrm{GeV}$.

The absolute atmospheric neutrino flux is known with an error of $20-30 \%$ essentially due to the uncertainty in the absolute flux of the primary cosmic rays [90,91]. The error decreases $(\sim 5 \%)$ for the ratio $\left(\nu_{\mu}+\bar{\nu}_{\mu}\right) /\left(\nu_{e}+\bar{\nu}_{e}\right)$. This is the main reason for the commonly used double ratio

$$
R=\left(\frac{\mu}{e}\right)_{D A T A} /\left(\frac{\mu}{e}\right)_{M C}
$$

where $\mu$ and $e$ represent the number of $\mu$-like and $e$-like detected events for real (DATA) and simulated (MC) neutrino interactions, respectively. In the absence of physics beyond the Standard Model one would expect $R=1$.

The distance $L$ travelled by neutrinos before reaching the detector is related to $\theta_{z}$ through the formula

$$
L=\sqrt{R_{\odot}^{2}+\left(R_{\odot}-d\right)^{2}\left(1-\cos ^{2} \theta_{z}\right)}-\left(R_{\odot}-d\right) \cos \theta_{z}
$$

where $R_{\odot}$ is the Earth radius $(\sim 6000 \mathrm{~km})$ and $d$ is the average production height $(\sim 10 \mathrm{~km})$.

So far, the NUSEX [92], Frejus [93], IMB [94,95], Soudan2 [96], Kamiokande [97] and Super-Kamiokande [15] experiments have performed a measurement of the double ratio $R$. The experimental status is summarised in Table 5. Two experiments, Frejus and NUSEX, do not observe any anomaly, while IMB, 
Kamiokande, Soudan2 and Super-Kamiokande detect a significant statistical deviation of $R$ from unity. However, due to their poor statistics, NUSEX and Frejus are compatible within $2 \sigma$ with the other experiments.

\subsection{Zenith angle dependence and oscillation hypothesis}

If the deviation of $R$ from unity is due to neutrino oscillations one should expect it varying as a function of the neutrino path length $L$. Neutrinos produced in interactions above the detector are called downwardgoing and have a typical path length of $\sim 10 \mathrm{~km}$. Neutrinos produced on the opposite side of the Earth are defined upward-going and have a path length up to $12000 \mathrm{~km}$.

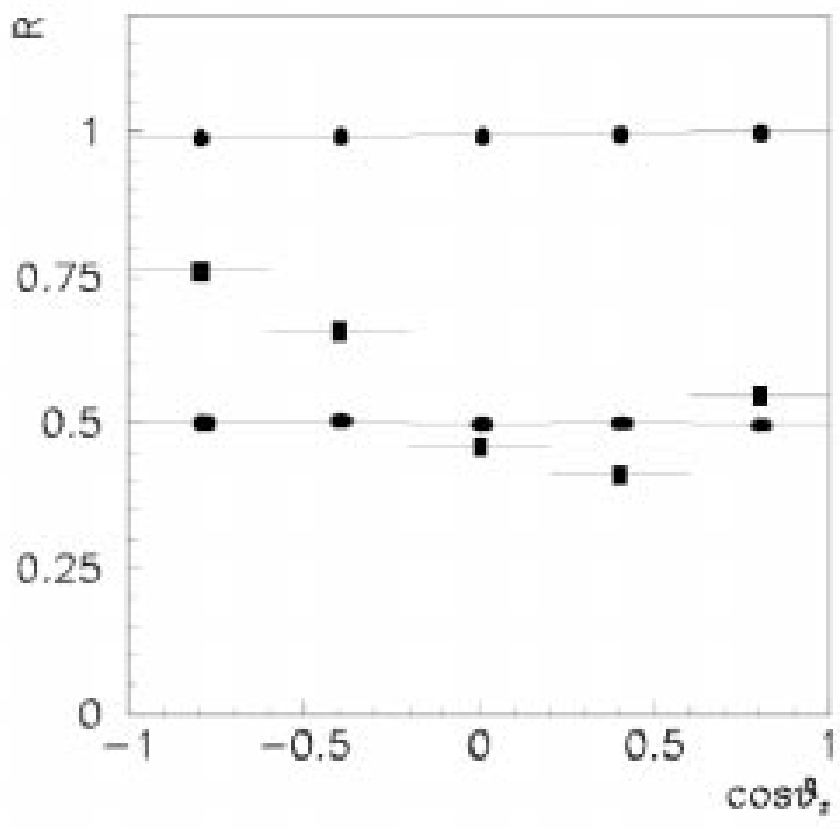

Figure 11: Expected value of $R$ (assuming pure $\nu_{\mu} \leftrightarrow \nu_{e}$ oscillations) as a function of $\cos \theta_{z}$ for $1.5 \mathrm{GeV}$ neutrinos and for different $\Delta m^{2}$ values (circle $=10^{-5} \mathrm{eV}^{2}$, rectangle $=10^{-3} \mathrm{eV}^{2}$, ellipse $=10^{-1} \mathrm{eV}^{2}$ ).

The path length may be expressed in terms of $\cos \theta_{z}$ (Eq. (39)). An example of the $R$ vs $\cos \theta_{z}$ dependence for $1.5 \mathrm{GeV} \nu_{\mu}$ is given in Fig. 11 for different values of $\Delta m^{2}$ and assuming pure $\nu_{\mu} \leftrightarrow \nu_{e}$ oscillations. The oscillation probability is small for very low $\Delta m^{2}$ values $\left(\sim 10^{-5} e V^{2}\right)$. Therefore, the $R$ value is expected to be close to one regardless the value of $L$. For large $\Delta m^{2}$ values $\left(\sim 10^{-1} e V^{2}\right)$ one half of the $\nu_{\mu}$ oscillates, again independently of the path length. The expected value of $R$ is then nearly 0.5. For intermediate values of $\Delta m^{2}\left(\sim 10^{-3} \mathrm{eV}^{2}\right)$ one expects $R$ varying as a function of $L$. Neutrino oscillations could then be identified by detecting the zenith angle dependence of $R$.

The first evidence for a zenith angle dependence was reported by the Kamiokande Collaboration. The 

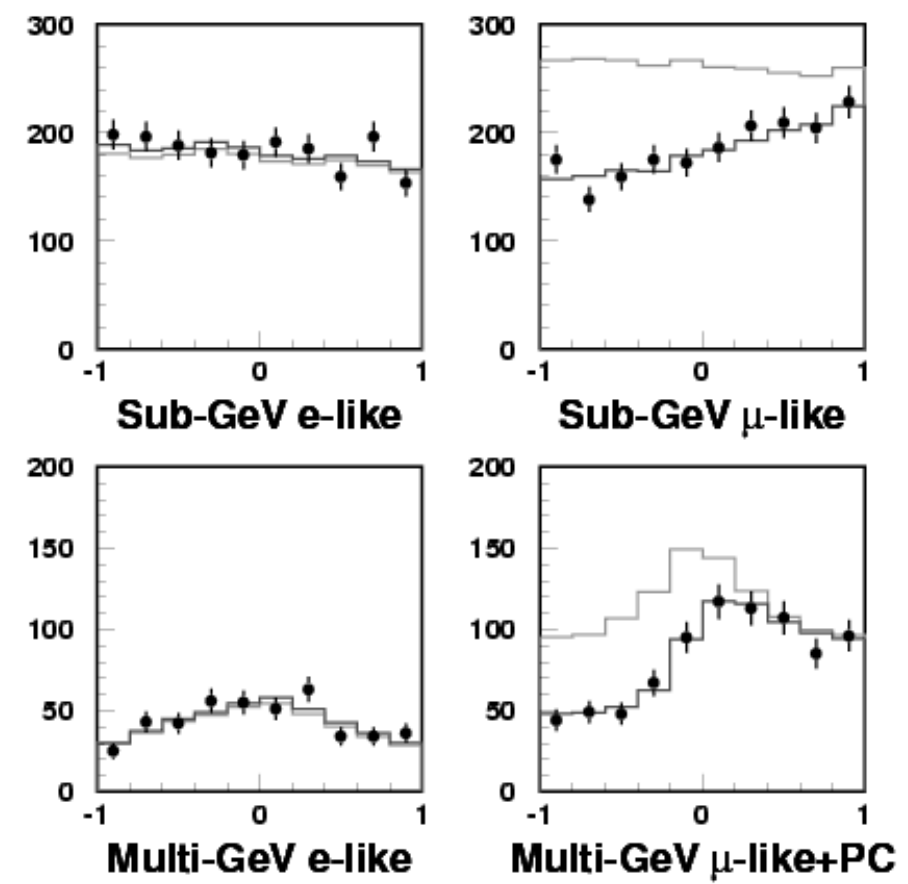

Figure 12: Super-Kamiokande zenith angle distributions for $\mu$-like and $e$-like events for $s u b-G e V$ and multi-GeV data. The grey line shows the Monte Carlo expectation in the absence of oscillations. The bold line indicates the fit result (given in the text). 
interpretation of the effect in terms of $\nu_{\mu} \leftrightarrow \nu_{e}$ oscillations has been ruled out by the CHOOZ result $[17,18]$. The best fit to the data in terms of $\nu_{\mu} \leftrightarrow \nu_{\tau}$ oscillations gives the following values for the oscillation parameters: $\Delta m^{2}=1.6 \times 10^{-2} e V^{2}$ and $\sin ^{2} 2 \theta=1$ [97]. Super-Kamiokande has confirmed the Kamiokande result with a much higher statistics making a strong claim for the observation of neutrino oscillations [15]. In agreement with the CHOOZ result, no anomaly has been observed for $\nu_{e}$.

The events in the Super-Kamiokande detector are subdivided in two classes: fully contained (FC) and partially contained (PC) events. For FC events all the energy is deposited in the fiducial volume of the detector. Conversely, PC events have at least one particle exiting the fiducial volume. FC events are further subdivided into sub-GeV $\left(E_{v i s}<1.33 \mathrm{GeV}\right)$ and multi-GeV $\left(E_{v i s}>1.33 \mathrm{GeV}\right)$ samples. Only single-ring events ${ }^{1}$ are retained for the analysis. For $\mathrm{PC}$ events no single-ring requirement is made.

The distributions of electron-like and muon-like events as a function of $\cos \theta_{z}$ are shown in Fig. 12. The grey line indicates the predicted fluxes without oscillations. No anomaly is observed in fact for $\nu_{e}$. The bold line shows the result of a fit which assumes $\nu_{\mu} \leftrightarrow \nu_{\tau}$ oscillations. The best fit for FC and PC events gives $\Delta m^{2}=3.05 \times 10^{-3} \mathrm{eV}^{2}$ and $\sin ^{2} 2 \theta=0.995$ with a $\chi^{2} / D O F=55.4 / 67$ [15]. The possibility of $\nu_{\mu} \leftrightarrow \nu_{\text {sterile }}$ oscillations is contemplated but is disfavoured at the $95 \%$ C.L. by Super-Kamiokande [15].

The above results have been confirmed by the zenith angle dependence of upward muons produced by $\nu_{\mu}$ and $\bar{\nu}_{\mu}$ and interacting with the rock surrounding the detector [98].

A combined analysis of all Super-Kamiokande measurements (sub-GeV, multi-GeV and upward-going muons) has been performed [15]. The best fit indicates $\Delta m^{2}=3.5 \times 10^{-3} \mathrm{eV}^{2}$ and $\sin ^{2} 2 \theta=1$ $\left(\chi^{2} /\right.$ d.o.f. $\left.=67.5 / 82\right)$ constraining the oscillation parameters at the $90 \%$ C.L. in the region with $\sin ^{2} 2 \theta>$ 0.84 and $\Delta m^{2}$ ranging from 2 to $6 \times 10^{-3} \mathrm{eV}^{2}[15]$.

The Soudan2 experiment exploits a 1 kton fine-grained tracking calorimeter for the detection of atmospheric neutrinos. The experiment observes a smaller than expected $R$ value but with a less pronounced zenith dependence [96] than Super-Kamiokande. Although with low statistics (Table 5), Soudan2 has a better angular resolution than Super-Kamiokande. The best fit to the $L / E$ distribution gives oscillation parameters $\Delta m^{2}=8 \times 10^{-3} e V^{2}$ and $\sin ^{2} 2 \theta=0.95$.

An analysis of the atmospheric-neutrino zenith angle dependence has also been performed by the MACRO experiment at Gran Sasso [99] which makes use of a massive calorimeter. The best fit to the $L / E$ distribution gives oscillation parameters consistent with the Super-Kamiokande results.

The allowed regions for Soudan2, MACRO, Kamiokande and Super-Kamiokande are shown in Fig. 13. Possible interpretations of the atmospheric neutrino deficit together with the other evidences for neutrino oscillations are discussed in Section 11.

\section{Neutrino beams from particle accelerators}

\subsection{General features}

B. Pontecorvo [100] and M. Schwartz [101] proposed independently in the late fifties the idea of accelerator neutrino beams produced by the decay of $\pi$ and $K$ created in the interactions of primary protons with a suitable target

\footnotetext{
${ }^{1}$ A Cerenkov ring image corresponds to a single charged particle above the threshold.
} 


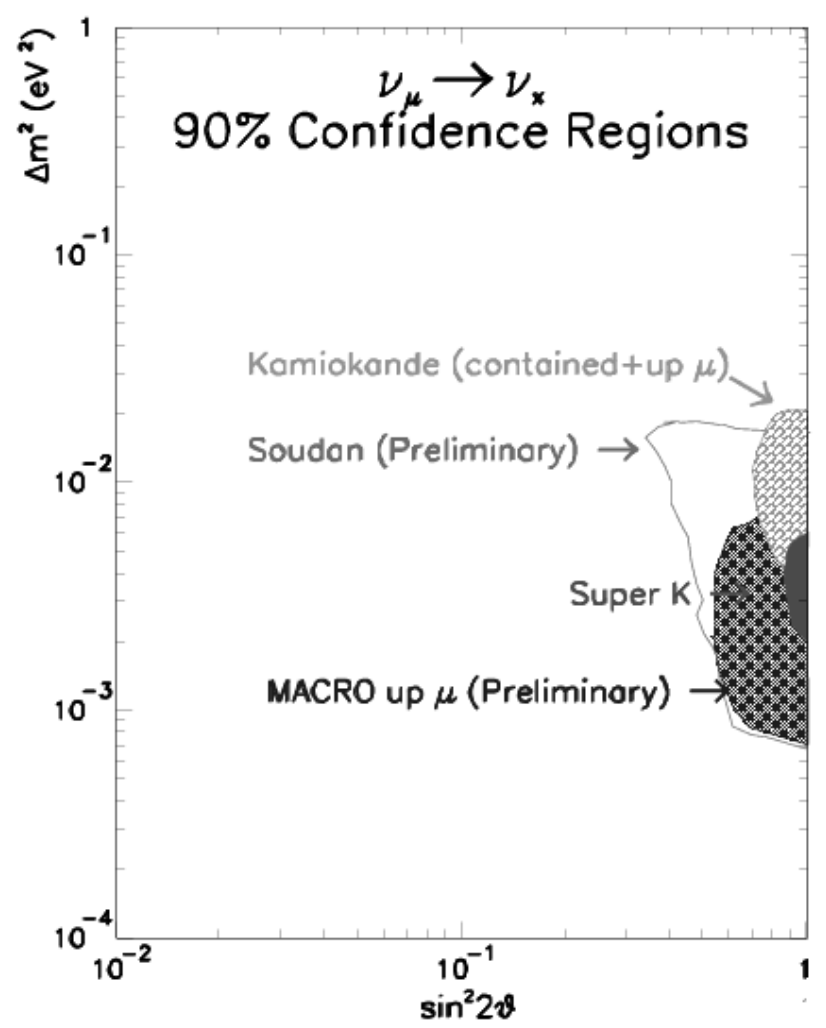

Figure 13: Atmospheric neutrinos: summary of the allowed parameter regions for Soudan2, MACRO, Kamiokande and Super-Kamiokande. 


$$
\begin{aligned}
& \pi^{+(-)} \rightarrow \mu^{+(-)}+\nu_{\mu}\left(\bar{\nu}_{\mu}\right) \\
& K^{+(-)} \rightarrow \mu^{+(-)}+\nu_{\mu}\left(\bar{\nu}_{\mu}\right)
\end{aligned}
$$

The first beam was actually built at the Brookhaven National Laboratory in the early sixties and was used for the classical experiment which demonstrated that $\nu_{e} \neq \nu_{\mu}[102]$. A few years later a neutrino beam was realised at CERN for the systematic study of neutrino properties $[103,104]$.

One can identify the main components of a neutrino beam line at a high energy accelerator as

- the target onto which protons are sent to produce pions and kaons;

- the focusing system which guides the mesons along the desired neutrino beam direction;

- the decay tunnel (usually evacuated) where mesons decay and produce neutrinos and muons;

- the absorber made of (magnetised) iron, earth and other shielding material. Its task is to range out or sweep out the muons and to absorb the protons which did not interact in the target as well as the mesons which did not decay.

From meson decay kinematics it follows that the neutrino energy is given by

$$
E_{\nu}=\frac{m_{\pi(K)}^{2}-m_{\mu}^{2}}{m_{\pi(K)}^{2}} \frac{E_{\pi(K)}}{\left(1+\gamma^{2} \theta^{2}\right)}
$$

where $\gamma$ is the Lorentz boost of the parent meson, $E_{\pi(K)}$ its energy and $\theta$ the angle of the neutrino with respect to the meson flight direction. The angular divergence of the beam is due to the transverse momentum $\left(p_{\perp \nu}\right)$ of the decay and parent particles. The first contribution is unavoidable since the maximum $p_{\perp \nu}$ in a pion (kaon) decay is 29.8 (236) $\mathrm{MeV}$. The second one can be reduced by optimising the meson focusing system.

In the following Sections we describe some accelerator neutrino beams suited for (or dedicated to) neutrino oscillation searches.

\subsection{The CERN WANF Wide Band Beam}

The West Area Neutrino Facility (WANF) of the CERN Super Proton Synchrotron (SPS) has been operating continuously from 1976 to 1998 with only minor interruptions for changes in the beam configuration. For many years it has provided a very intense beam of high-energy muon neutrinos. We refer to [105] for a detailed description of the WANF beam in the configuration used until the end of the eighties.

The main components of the WANF upgraded in 1992 [106] for the CHORUS and NOMAD experiments are shown in Fig. 14. Protons extracted from the SPS at $450 \mathrm{GeV}$ are directed towards the West Experimental Area and focused onto a beryllium target. A fast-slow resonant extraction produces two beam spills of $6 \mathrm{~ms}$ duration and separated by $2.7 \mathrm{~s}$ for each $14.4 \mathrm{~s}$ cycle of the accelerator. The time structure of the SPS cycle is shown in Fig. 15.

Pions and kaons produced by protons hitting the target and in the required energy band are focused towards the experiments giving a roughly parallel beam. Two co-axial magnetic lenses known as horn and 
TOP VIEW of neutrino cave

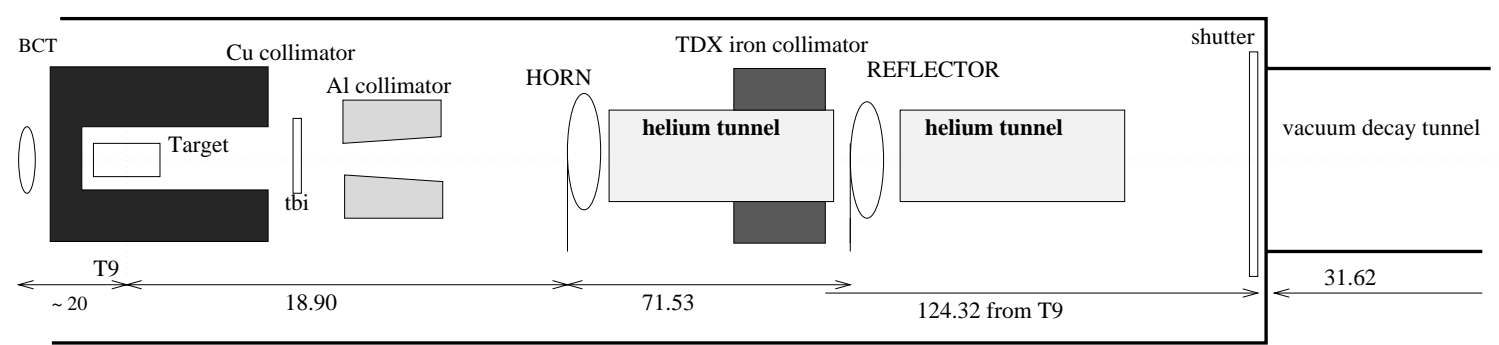

Muon counting Pits

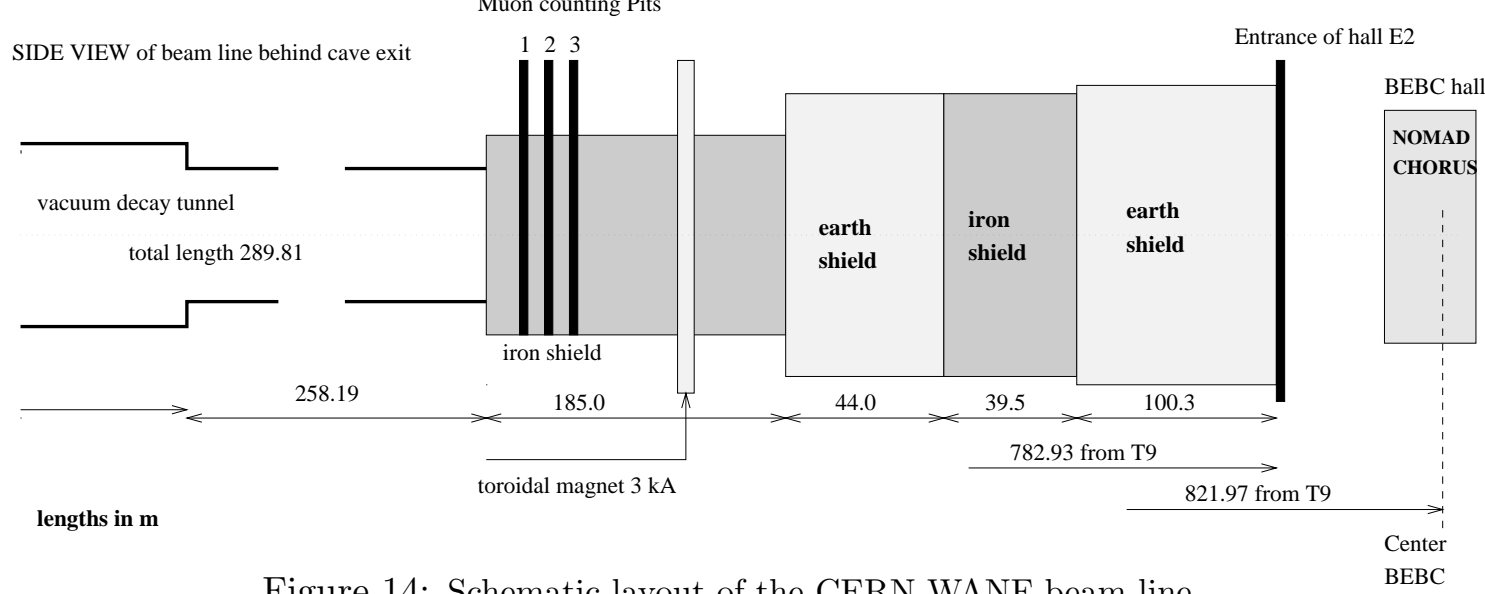

Figure 14: Schematic layout of the CERN WANF beam line.

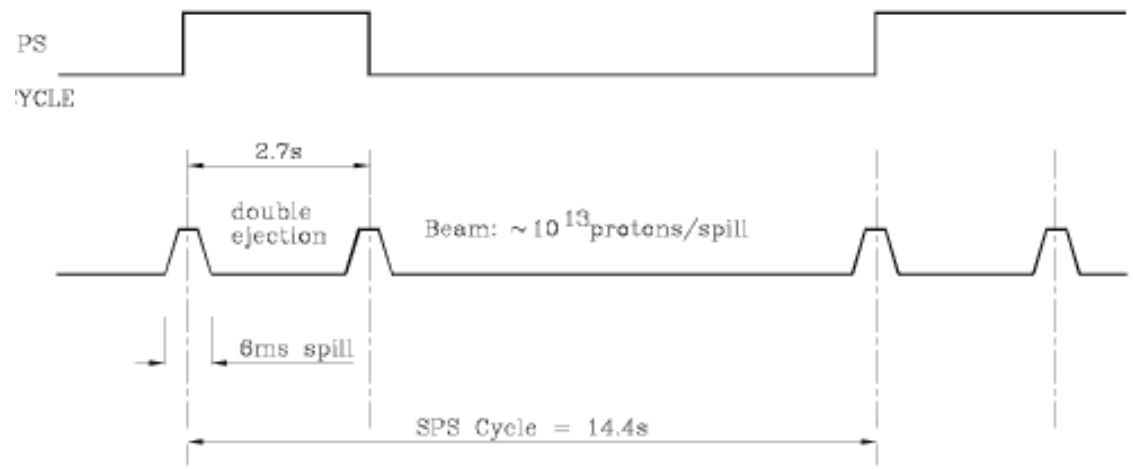

Figure 15: The time structure of the CERN SPS cycle and of the WANF. 
reflector are used. Pions and kaons can thus decay in flight and produce muons and muon neutrinos. Muons and not interacting hadrons are stopped by an iron and earth shielding at the end of the decay path. Only neutrinos are left. The detectors are placed $\sim 800 \mathrm{~m}$ downstream of the target.

In the $120 \mathrm{~m}$ long cavern the path between the focusing devices is filled with a helium bag at atmospheric pressure in order to minimise the number of parent mesons interacting before their decay. The final $300 \mathrm{~m}$ of the decay tunnel are evacuated to 0.5 Torr. The measurement of the muon flux distributions at a number of monitoring gaps in the iron shielding provides a tool to determine the neutrino flux and to align the beam.

The composition of the WANF beam used by the CHORUS and NOMAD experiments since 1994 is a quasi-pure $\nu_{\mu}$ Wide Band Beam (WBB) with average energy of $27 \mathrm{GeV}$. A few percent contamination of $\bar{\nu}_{\mu}$ and about $1 \%$ of $\left(\nu_{e}+\bar{\nu}_{e}\right)$ is present. Fig. 16 shows the energy spectra for the different components of the WANF WBB at the location of the CHORUS detector target [107].

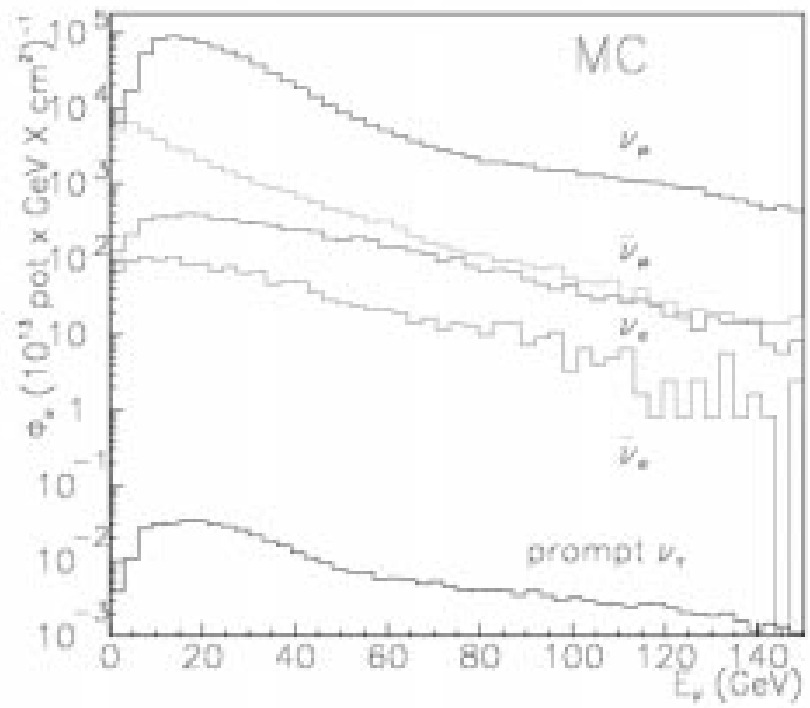

Figure 16: Simulated energy spectra of the different neutrino components of the WANF WBB at the location of the CHORUS target (transverse dimension of $1.42 \times 1.44 \mathrm{~m}^{2}$ ).

Another possible neutrino beam configuration is the so-called Narrow Band Beam (NBB). The main difference between a WBB and a NBB is given by the narrower neutrino energy distribution of the latter. This can be obtained by selecting a small momentum bite of the parent $\pi$ and $K$ and rejecting (mainly) low momentum mesons. The neutrino yield is obviously reduced, which is an important drawback for oscillation searches.

The advantage of a NBB configuration is the possibility to obtain a rather precise estimate of the neutrino energy. This is reflected in a better knowledge of the neutrino vertex kinematics being neutrinos practically monoenergetic. In addition, the rejection of wrong sign parents is more effective in a NBB. The momentum selection of pions and kaons is obtained by means of momentum splits, dipoles and quadrupoles, which replace the horn and reflector magnetic lenses of the WBB. A typical momentum bite for the parent selection in a NBB is $\Delta p / p \sim 5-10 \%$. For details about NBB designs we refer to [105,108]. 


\subsection{The CERN-Gran Sasso CNGS beam}

The experimental results on the atmospheric neutrino anomaly motivated the design of a series of facilities able to provide neutrino beams for LBL experiments. The first LBL beam to be constructed and operational has been the Japanese beam from Kamioka to the Super-Kamiokande detector (Section 6.5). Another beam (NuMI) is under construction in the USA, from Fermilab to the MINOS detector in the Soudan mine (Section 6.4).

As an example of facilities of this type, we describe in some detail the CNGS beam [109] from the CERN SPS towards the Gran Sasso Laboratory located underground in central Italy $732 \mathrm{~km}$ from CERN. The project has recently been approved by CERN and INFN. Its commissioning is expected in the year 2005 .

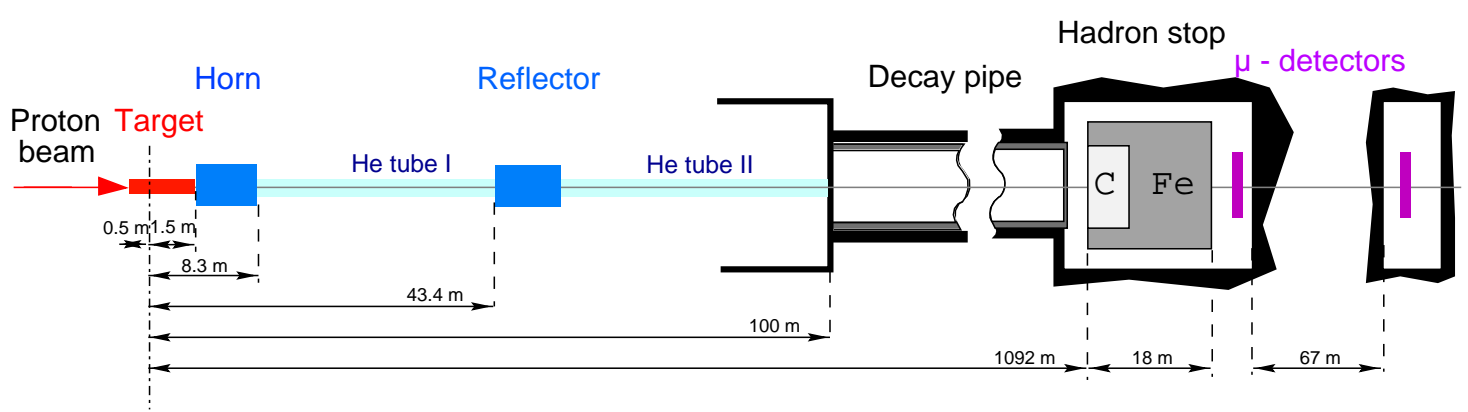

Figure 17: Layout of the components of the CNGS neutrino beam line.

Similar to the WANF, the CNGS neutrino beam is produced by the decay of $\pi$ and $K$ mesons originating from the high energy, high intensity SPS proton beam hitting a production target. The main components of the beam line are schematically shown in Fig. 17.

The SPS accelerator is being upgraded for its future role of Large Hadron Collider (LHC) injector while continuing to deliver protons and ions for fixed target experiments. For the purpose of the CNGS it is important that one of the extractions from the SPS towards the LHC points roughly in a southern direction. The extraction system foreseen for the LHC allows fast-extracted pulses (of up to $20 \mu s$ ) to be sent to the TI8 transfer line towards the LHC. This system can also be used to extract protons onto the CNGS target with some modifications needed to allow two fast extractions per cycle.

The total length of the proton beam from the SPS to the CNGS target is $830 \mathrm{~m}$ and its maximum design momentum $450 \mathrm{GeV}$ (the maximum SPS energy). The beam branches off the TI8 beam after $110 \mathrm{~m}$. The deflection (horizontal and vertical) needed to reach the direction towards Gran Sasso is achieved by 73 bending magnets. This is illustrated in Fig. 18.

The target foreseen for the CNGS is based on the successful design of the target used for the WANF. 13 rods of graphite $10 \mathrm{~cm}$ long and $4 \mathrm{~mm}$ diameter each are placed in a cast aluminium container to form a $2 \mathrm{~m}$ long target array. The rods are cooled by a forced helium flow. The change from beryllium (WANF) to graphite has been mainly motivated by the change from fast-slow $(6 \mathrm{~ms})$ to fast $(10 \mu \mathrm{s})$ proton beam extraction and by the better thermo-mechanical properties expected for graphite.

The WANF experience is at the origin of the choice for the focusing elements, horn and reflector, two coaxial lenses similar in shape but different in size. Their characteristics are analogous to those of the WANF 


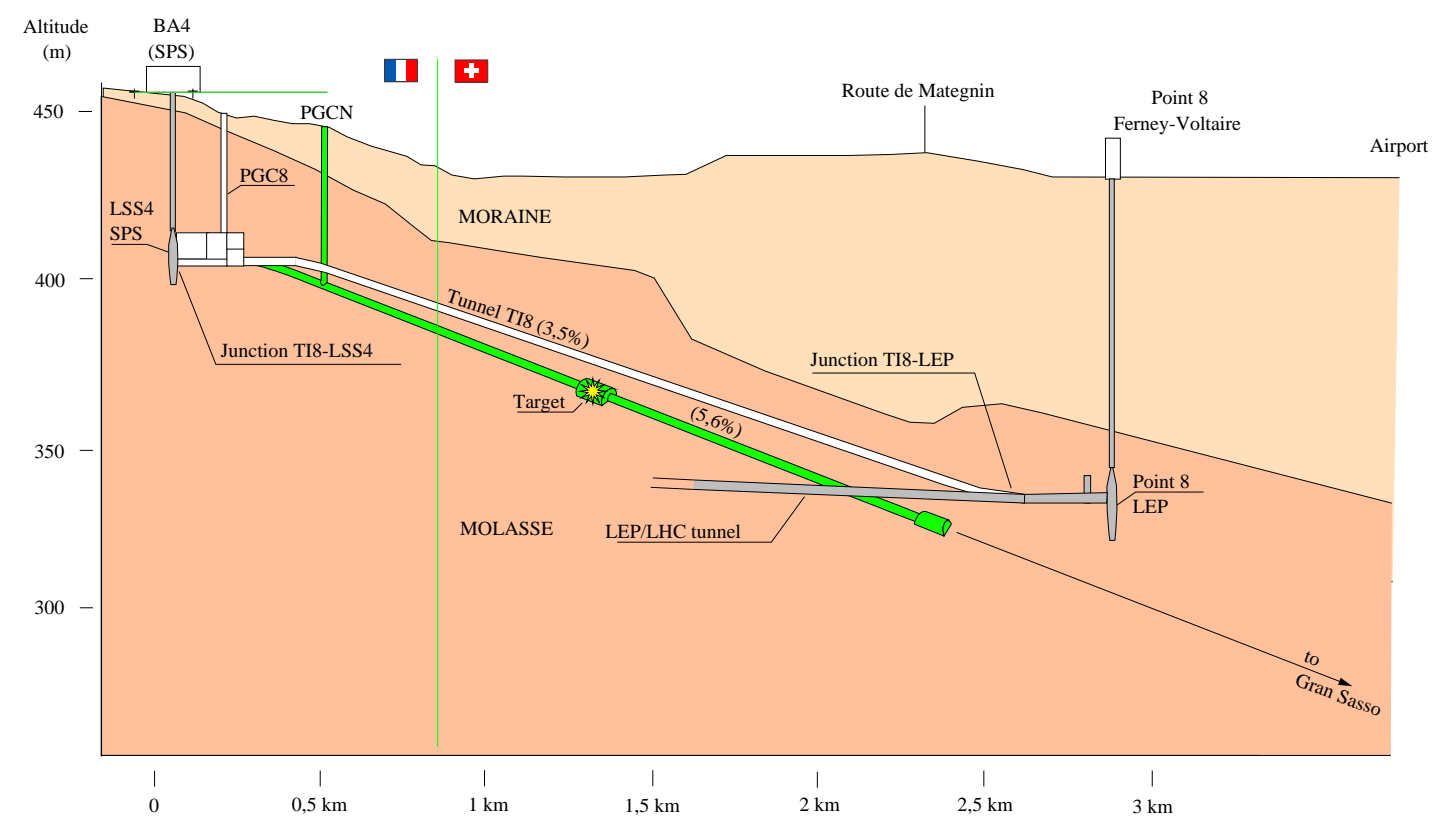

Figure 18: Vertical cut of the CNGS beam line.

\begin{tabular}{||c|c||}
\hline & Gran Sasso location \\
\hline \hline$\nu_{\mu}\left(m^{-2} /\right.$ pot $)$ & $4.39 \times 10^{-9}$ \\
\hline Mean $\nu_{\mu}$ energy $(\mathrm{GeV})$ & 26.7 \\
\hline$\nu_{\mu}$ CC events $/\left(10^{19}\right.$ pot kton $)$ & 473 \\
\hline$\nu_{e} / \nu_{\mu}$ & $0.8 \%$ \\
\hline $\bar{\nu}_{\mu} / \nu_{\mu}$ & $2.2 \%$ \\
\hline $\bar{\nu}_{e} / \nu_{\mu}$ & $0.08 \%$ \\
\hline \hline
\end{tabular}

Table 6: Features of the original CNGS reference beam; pot stands for protons on target.

lenses. The distance of horn and reflector from the target as well as the shape of the magnetic field are chosen to optimise a wide-band high-energy $\nu_{\mu}$ beam.

The typical $\pi$ decay lengths $(2.2 \mathrm{~km}$ at $40 \mathrm{GeV})$ make a long decay tunnel desirable. Given the angular distribution of the parent mesons which can produce neutrinos, the longer the decay tunnel the larger has to be its diameter. A tunnel of $2.45 \mathrm{~m}$ diameter and $1000 \mathrm{~m}$ length has been chosen for the CNGS. A massive iron beam dump is situated at the exit of the decay tunnel. A near detector would thus be shielded against muons.

Signals induced by muons from meson decays in two arrays of silicon detectors placed in the hadron stopper allow on-line monitoring and tuning of the beam (steering of the proton beam on target, horn and reflector alignment, etc.). The separation of the two arrays, equivalent to $25 \mathrm{~m}$ of iron, allows a rough measurement of the muon energy spectrum and of the beam angular distribution.

A reference beam line design has been produced [109] with the main aim to define the civil engineering work and serve as a basis for optimisation dictated by the physics programme. Mainly the proton energy, the 
extraction from the SPS, the target room design, the geometry of the decay tunnel and the beam absorber are chosen. The expected characteristics of the CNGS reference beam are derived by means of a full Monte Carlo simulation based on the FLUKA98 package [110,111]. These features are presented in Table 6 and in Fig. 19 which show the $\nu_{\mu}$ energy spectrum at Gran Sasso as given in [109].

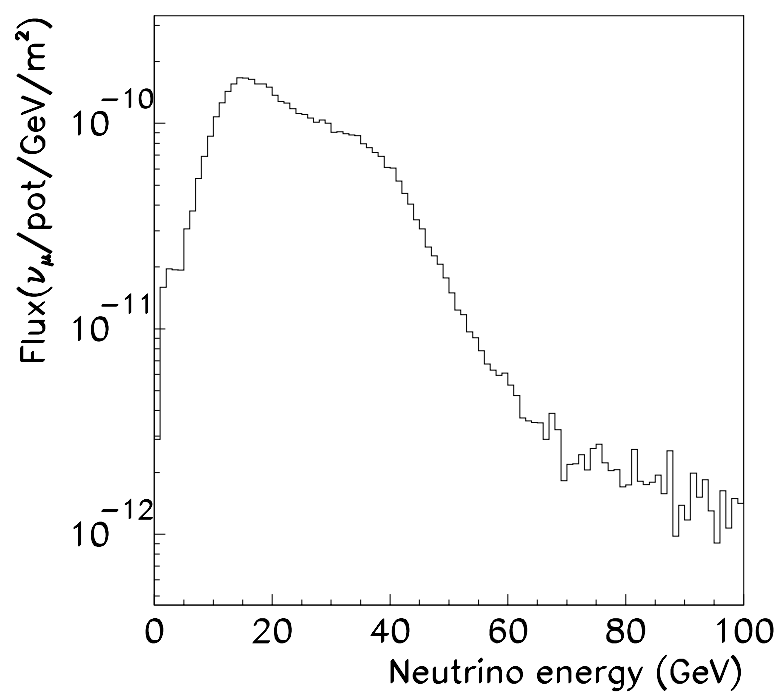

Figure 19: Energy spectrum of the $\nu_{\mu}$ flux of the CNGS reference beam at Gran Sasso.

In the framework of the reference beam design, simulations of the complete process of production of neutrinos from $400 \mathrm{GeV}$ SPS protons indicate that the expected flux of $\nu_{\mu}$ is $4.4 \times 10^{-9}$ pot $^{-1} \mathrm{~m}^{-2}$ at the Gran Sasso laboratory. The average energies of the $\nu_{\mu}$ are $\sim 24 \mathrm{GeV}$ and $\sim 27 \mathrm{GeV}$ for the near and far location, respectively. A contamination of about of $2 \% \bar{\nu}_{\mu}$ and slightly less than $1 \%\left(\nu_{e}+\bar{\nu}_{e}\right)$ is estimated. The rate of $\nu_{\mu}$ CC interactions (events per proton and per ton of detector material) is expected to be about $4.7 \times 10^{-20}$ at the Gran Sasso location. In the following, we present the improvements obtained by a subsequent beam optimisation.

\subsubsection{Optimisation for $\nu_{\mu} \leftrightarrow \nu_{\tau}$ oscillation searches}

The experimental programme at the CNGS is focused towards $\nu_{\mu} \leftrightarrow \nu_{\tau}$ appearance. The original CNGS reference beam [109] is not far from optimum for such a search. However, an optimisation of the target/horn/reflector system could yield a gain in flux of oscillated neutrinos [112]. Another aspect related to the beam optimisation is the loss of flux due to secondary particle interactions in the material along the beam line. In the CNGS reference beam design this reduces the neutrino flux by about one half, as shown in Fig. 20. The effort needed in order to recover from this loss is worthwhile, given the cost of the detector mass necessary to compensate for the lower beam intensity.

Since the appearance of the $\nu_{\tau}$ is detected through its CC interaction with the experimental target, its 


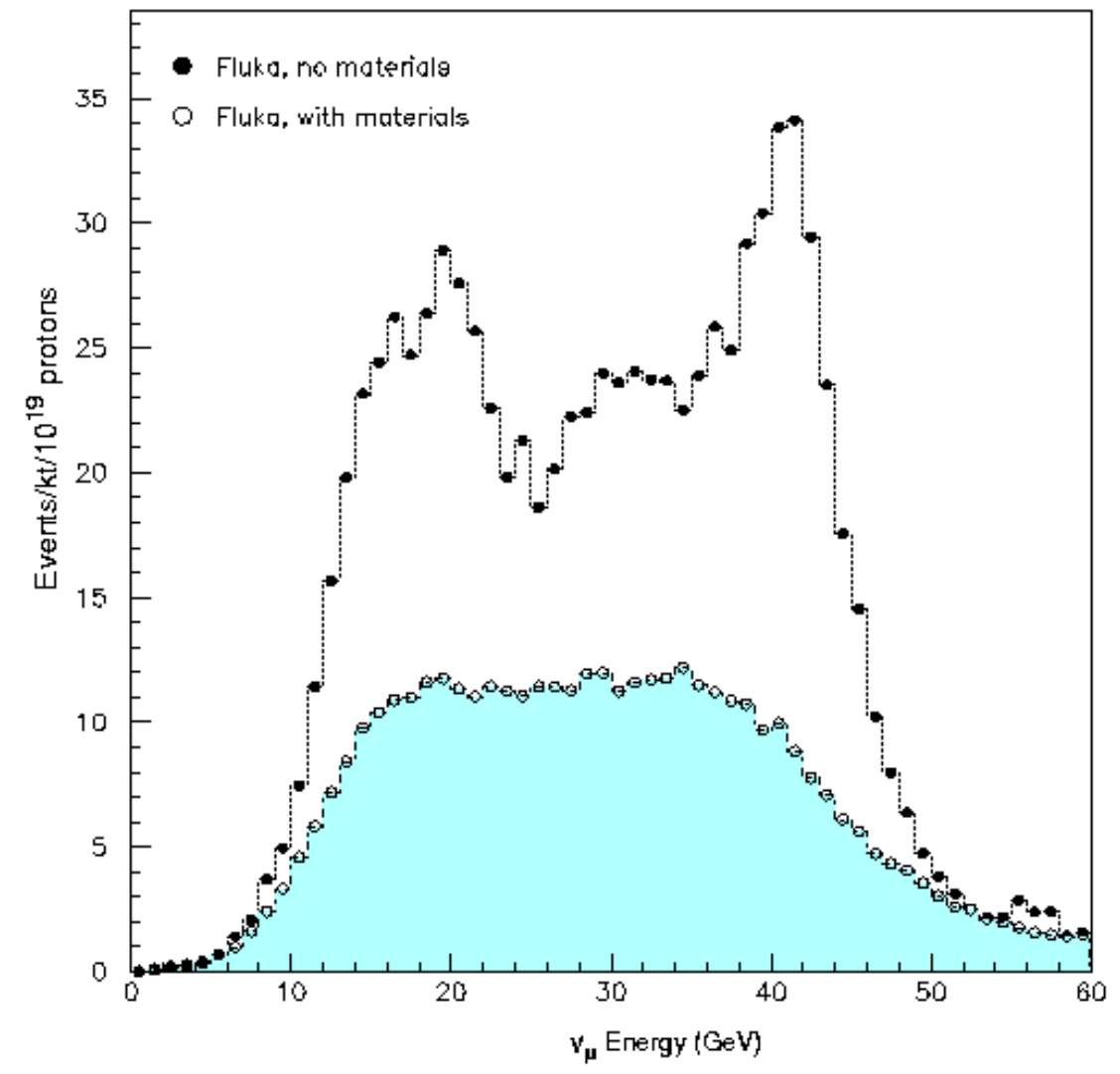

From the desk of (Your Name Here)

Figure 20: Comparison of the number of $\nu_{\mu} \mathrm{CC}$ events at Gran Sasso with and without interaction of secondary particles with material along the beam line (as a function of the neutrino energy). 
energy must be sufficient to produce its $\tau$ partner. This sets a lower limit on the energy of the neutrino beam. The threshold to produce a $\tau$ is $\sim 3.5 \mathrm{GeV}$ and the ratio of cross-sections $\left(\sigma_{\tau} / \sigma_{\mu}\right)$ increases slowly. High energy beams are therefore more suitable for a $\nu_{\mu} \leftrightarrow \nu_{\tau}$ appearance search. On the other hand, the fact that the expected value of $\Delta \mathrm{m}^{2}$ as indicated by Super-Kamiokande is of the order of $10^{-3} \mathrm{eV}^{2}$ leads to a somehow lower energy of the $\nu_{\mu}$ oscillating into $\nu_{\tau}$, as shown in the following.

The number of $\tau$ leptons observed in a given detector is determined by the relation

$$
N_{\tau}=N_{a} M_{d} \int \phi_{\mu}(E) P\left(\nu_{\mu} \leftrightarrow \nu_{\tau}\right)(E) \sigma_{\tau}(E) \epsilon(E) \mathrm{d} E
$$

where $\phi_{\mu}$ is the $\nu_{\mu}$ flux at the detector site, $P\left(\nu_{\mu} \leftrightarrow \nu_{\tau}\right)(E)$ is the oscillation probability between $\nu_{\mu}$ and $\nu_{\tau}, \sigma_{\tau}$ is the $\nu_{\tau}$ CC interaction cross-section, $\epsilon$ is the experimental $\tau$ detection efficiency, $N_{a}$ is the Avogadro number and $M_{d}$ is the detector mass. In Fig. $21 \sigma_{\tau}$ and $\sigma_{\mu}$ are plotted as a function of $E$ together with their ratio. In Fig. $22 P(E)$ is shown in the case of $L=732 \mathrm{~km}$, full mixing and for different $\Delta \mathrm{m}^{2}$ values. One can see that $\sigma_{\tau}$ increases with increasing beam energy unlike the oscillation probability.
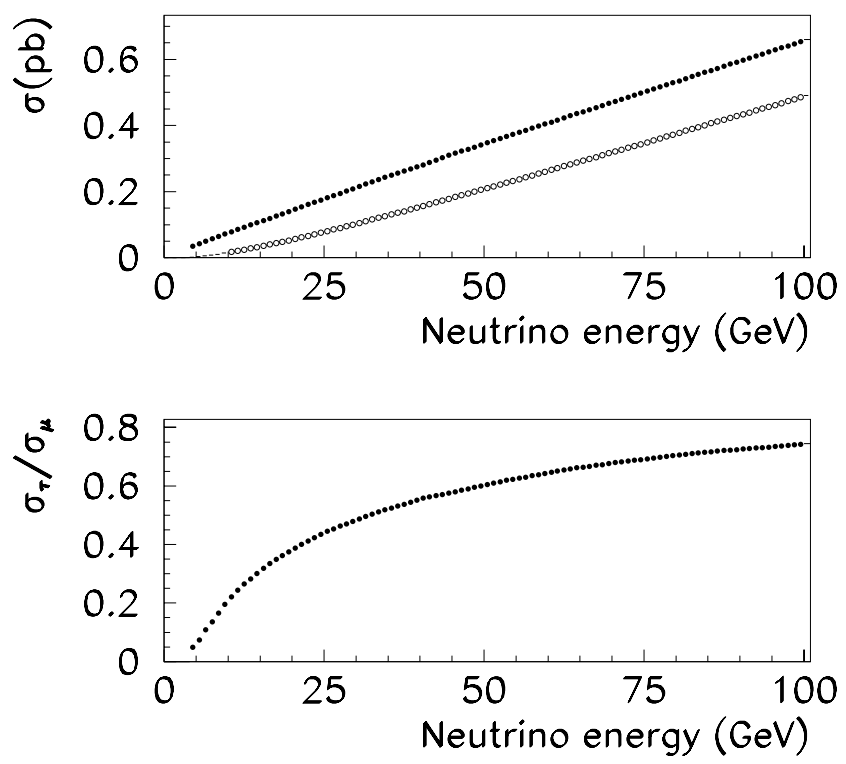

Figure 21: Top Figure: $\sigma_{\tau}(E)$ (open circles) and $\sigma_{\mu}(E)$ (black circles) as a function of the neutrino energy. Bottom Figure: the $\sigma_{\tau}(E) / \sigma_{\mu}(E)$ ratio. Deep-inelastic, quasi-elastic and resonance events have been included.

The $\nu_{\tau}$ cross-section can be parametrised as

$$
\sigma_{\tau}=\sigma_{0} E f_{\tau}(E)
$$

where $\sigma_{0}=0.67 \times 10^{-38} \mathrm{~cm}^{2} / \mathrm{GeV}$ and $f_{\tau}(E)$ is a function which incorporates the energy dependence. The number of $\nu_{\tau}$ interactions may be then expressed as 


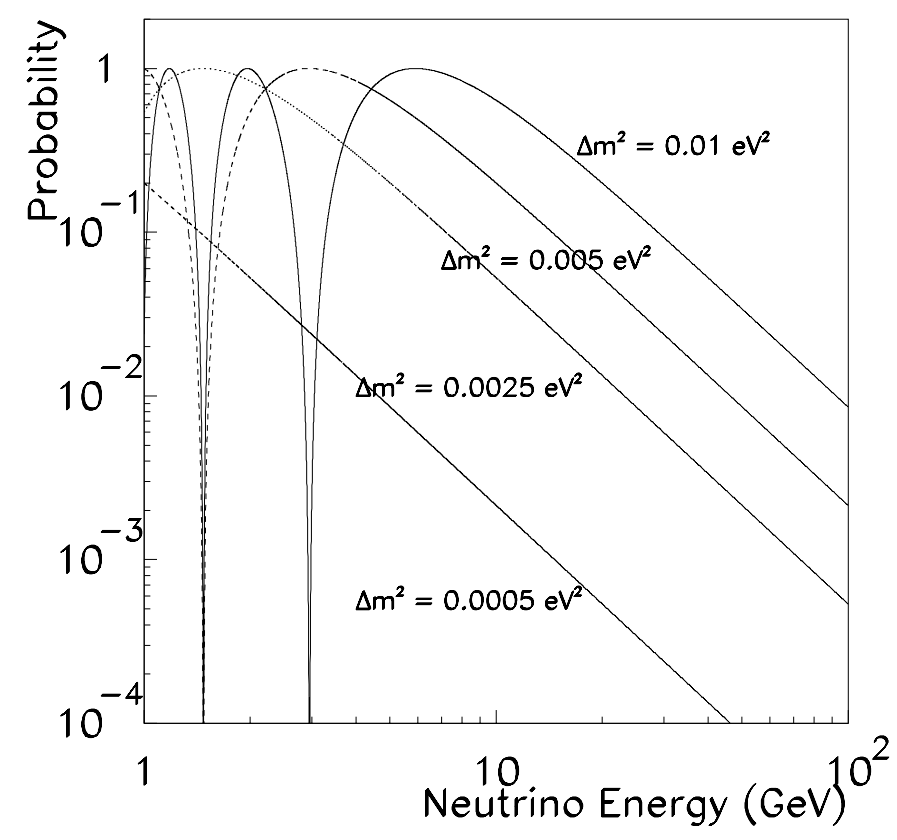

Figure 22: Oscillation probability (full mixing and $\mathrm{L}=732 \mathrm{~km}$ ) as a function of the energy for different $\Delta \mathrm{m}^{2}$ values.

$$
N_{\tau}=N_{a} M_{d} \sin ^{2} 2 \theta \sigma_{0} \int \phi_{\mu}(E) \sin ^{2}\left(\frac{1.27 L \Delta m^{2}}{E}\right) f_{\tau}(E) \epsilon(E) E d E
$$

If the condition $\Delta m^{2} L / E \ll 1$ holds (as in the case of small $\Delta m^{2}$ )

$$
N_{\tau} \sim N_{a} M_{d} \sin ^{2} 2 \theta\left(1.27 \Delta m^{2} L\right)^{2} \sigma_{0} \int \phi_{\mu}(E) f_{\tau}(E) \epsilon(E) d E / E
$$

As a consequence of that, the $\tau$ yield optimisation becomes independent of the actual $\Delta m^{2}$ value. One could think that the quantity to be maximised in order to take advantage of the long baseline configuration is the average of $f_{\tau}(E) \epsilon(E) / E$ over the beam energy spectrum. Fig. 23 shows the ratio $f_{\tau}(E) / E$ as a function of $E$. A neutrino beam peaked around $10 \mathrm{GeV}$ would seem to be the correct choice. However, this does not take into account the effect of the neutrino flux $\phi_{\mu}(E)$ and of the $\tau$ detection efficiency $\epsilon(E)$.

In Fig. 24 the energy spectrum of the CNGS beam is shown under the assumption of ideal focusing of all the mesons produced at the primary target. This approach of ideal focusing for optimisation purposes was originally introduced in [113]. If one convolutes the function $f_{\tau}(E) / E$ with the one of Fig. 24 the yield of $\nu_{\tau} \mathrm{CC}$ interactions steeply rises up to $\sim 10 \mathrm{GeV}$ and stays rather constant up to $20-25 \mathrm{GeV}$, as shown in Fig. 25. A real focusing system should be tuned according to the curve of Fig. 25.

\subsubsection{Beam line design}

There are two main components of the beam line which may be used to optimise the $\nu_{\tau}$ yield: the target and the focusing system. One can estimate to $\sim 5 \%$ the possible gain in neutrino flux obtainable by varying the 


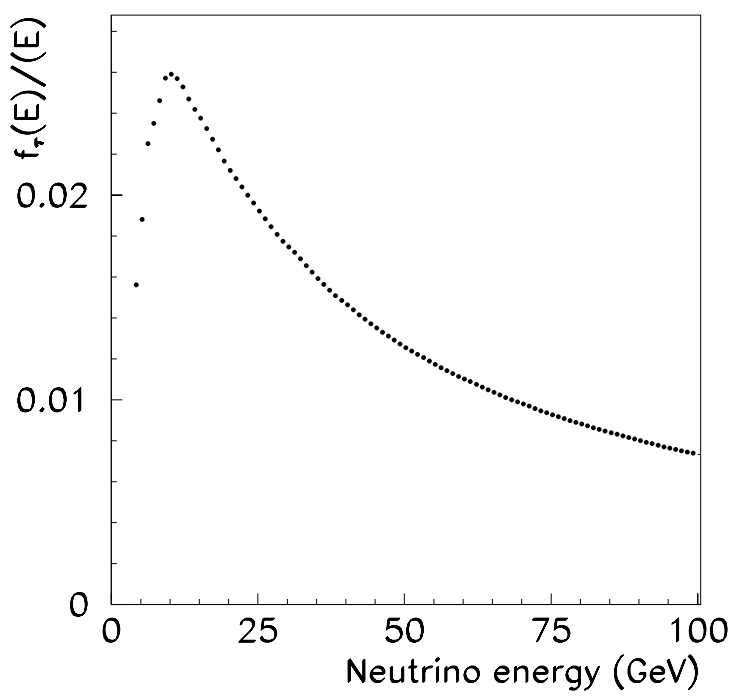

Figure 23: $f_{\tau}(E) / E$ as a function of $E$. Deep-inelastic, quasi-elastic and resonances are included.

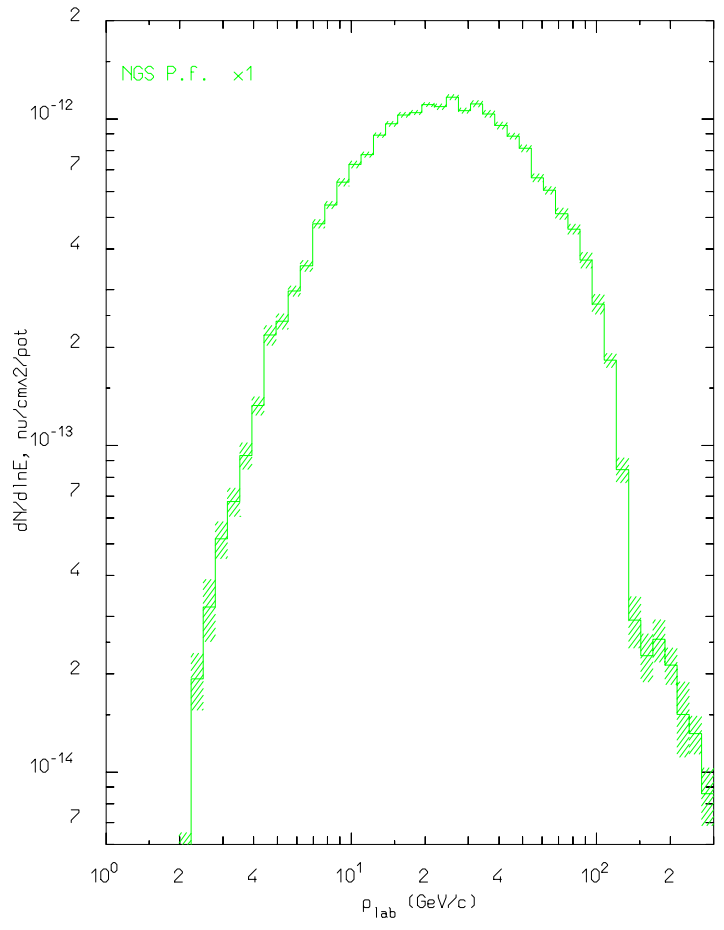

Figure 24: Neutrino fluxes at Gran Sasso for the CNGS beam in the hypothesis of ideal focusing. 


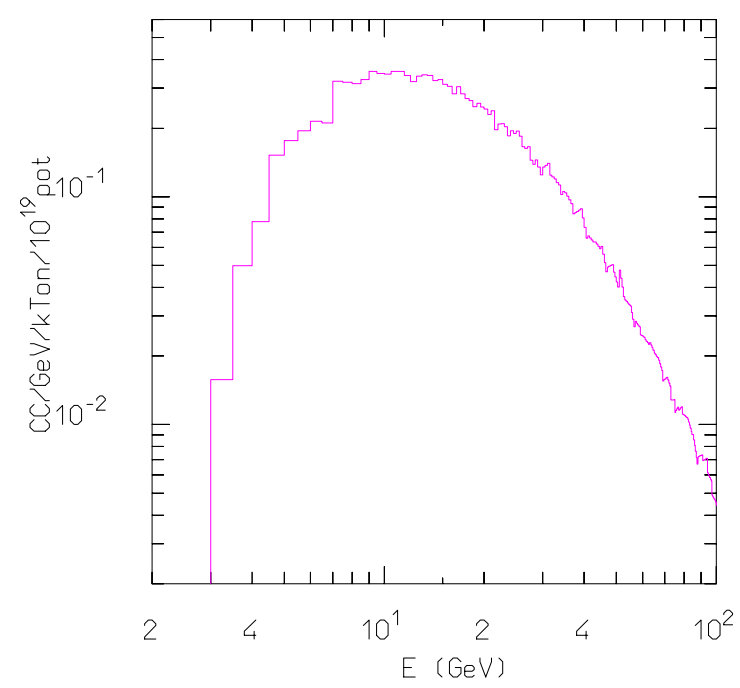

Figure 25: The function $\mathrm{dN}_{\tau} / \mathrm{dE}$ is plotted for $\Delta m^{2}=2.5 \times 10^{-3} \mathrm{eV}^{2}$ in the hypothesis of ideal focusing. This function is proportional to $\phi_{\mu}(E) f_{\tau}(E) / E$ in the limit of small $\Delta m^{2}$. Its shape is independent of $\Delta m^{2}$ while the total number of events scales with $\left(\Delta m^{2}\right)^{2}$. Note the logarithmic energy scale.

\begin{tabular}{||c|c|c|c|c||}
\hline & $\nu_{\mu}$ & $\bar{\nu}_{\mu}$ & $\nu_{e}$ & $\bar{\nu}_{e}$ \\
\hline \hline flux $\left(\nu /\left(10^{19}\right.\right.$ pot $\left.\left./ \mathrm{m}^{2}\right)\right)$ & $7.5 \times 10^{10}$ & - & - & - \\
\hline relative interaction rate & 1 & 0.02 & 0.008 & $5 \times 10^{-4}$ \\
\hline mean neutrino energy $(\mathrm{GeV})$ & 17 & - & - & - \\
\hline CC events $\left(\right.$ evts $/\left(10^{19}\right.$ pot $\times$ kton $\left.)\right)$ & 544 & 10.9 & 4.4 & 0.3 \\
\hline \hline
\end{tabular}

Table 7: Features of the CNGS beam optimised for $\nu_{\mu} \leftrightarrow \nu_{\tau}$ oscillation searches at the Gran Sasso location [115].

number of target rods and their relative spacing. In the reference beam design the nominal focusing energies are $50 \mathrm{GeV}$ and $80 \mathrm{GeV}$ for the horn and the reflector, respectively. With a parabolic horn/reflector shape the $30 / 50 \mathrm{GeV}$ configuration produces a gain of about $25 \%$ in $\tau$ yield w.r.t. the reference optics.

The optimisation for $\nu_{\mu} \leftrightarrow \nu_{\tau}$ oscillation searches outlined in the previous Section has led to the design of the new CNGS reference beam design developed by the CERN/INFN Technical Committee [114,115]. The resulting mean neutrino energy and the number of interactions for the different neutrino species of the new beam are given in Table 7. The expected yield of interacting $\nu_{\tau}$ computed for $\Delta m^{2}=3.5 \times 10^{-3} \mathrm{eV}^{2}$, for full mixing and with standard sets of neutrino cross-sections is $6.7 /\left(10^{19}\right.$ pot $\times$ kton $)$. Details on the updated CNGS beam design can be found in [115].

While high energy beams are preferred for $\nu_{\tau}$ appearance experiments, low energy neutrinos are better suited for $\nu_{\mu}$ disappearance searches. Two beams (LE1 and LE2) have been designed for the CNGS with a more compact target and larger geometrical acceptance relatively to the high energy beam [116]. In the case of LE1 (LE2) the horn and the reflector focus hadrons of 16 (10) $\mathrm{GeV}$ and 24 (15) GeV, respectively. A graphite target with $185 \mathrm{~cm}$ length and $3 \mathrm{~mm}$ diameter is assumed. The main features of the low energy beams are shown in Table 8. 


\begin{tabular}{||c|c|c||}
\hline$\sqrt{\left\langle E_{\nu_{\mu}}^{2}\right\rangle}(\mathrm{GeV})$ & $\phi_{\nu_{\mu}}\left(\nu /\left(10^{13} \mathrm{pot} / \mathrm{cm}^{2}\right)\right)$ & deep-inelastic CC $\left(\mathrm{evts} /\left(10^{19}\right.\right.$ pot $\left.\left.\times \mathrm{kton}\right)\right)$ \\
\hline \hline $8(6.8)$ & $3.45(2.29)$ & $169(104)$ \\
\hline \hline
\end{tabular}

Table 8: Main features of the CNGS LE1 (LE2) low energy beam.

Concerning the CNGS beam operation, a running scheme called shared [115] foresees an average of $4.5 \times 10^{19}$ pot/year. It presumes similar performance of the CERN accelerator complex as over the past years. The number of pot/year goes up to $7.6 \times 10^{19}$ with the so-called dedicated mode which assumes no SPS users other than the CNGS.

\subsection{The Fermilab-Soudan NuMI beam}

A similar beam facility dedicated to long baseline neutrino oscillation experiments has been designed and is under construction in the USA. The NuMI neutrino beam [117] will be produced by protons from the Fermilab Main Injector and will be directed towards the Soudan mine, $730 \mathrm{~km}$ away from the source as in the case of the CNGS. The beam is presently expected to be commissioned in the year 2003. The map of the neutrino beam direction is shown in Fig. 26.

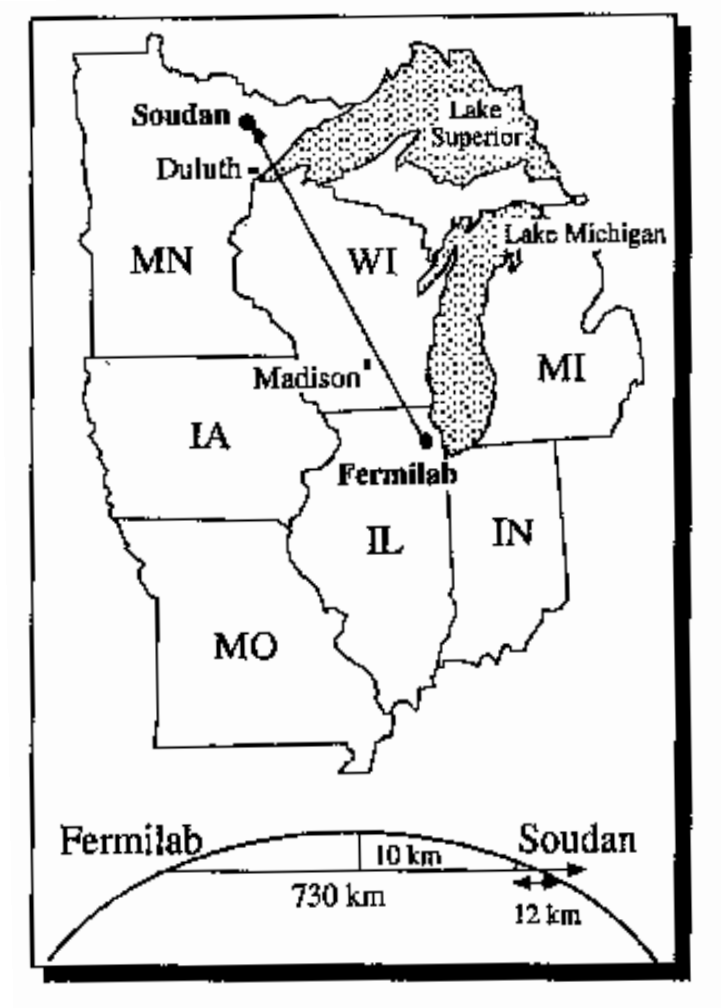

Figure 26: The trajectory of the MINOS neutrino beam from Fermilab to the Soudan mine.

The distinctive feature of the NuMI beam is the possibility to produce neutrinos with an energy spectrum 
which can be varied, with mean energies ranging from 1 to $20 \mathrm{GeV}$. This features is well adapted for disappearance searches and will allow to explore different $\Delta m^{2}$ domains. Since magnetic focusing systems have limited momentum acceptance, the different spectra can be obtained by changing the position of the horn and reflector along the beam line and adopting different targets. Three beams have been designed. Their characteristics are shown in Fig. 27 together with the spectrum corresponding to ideal focusing of the parent mesons.

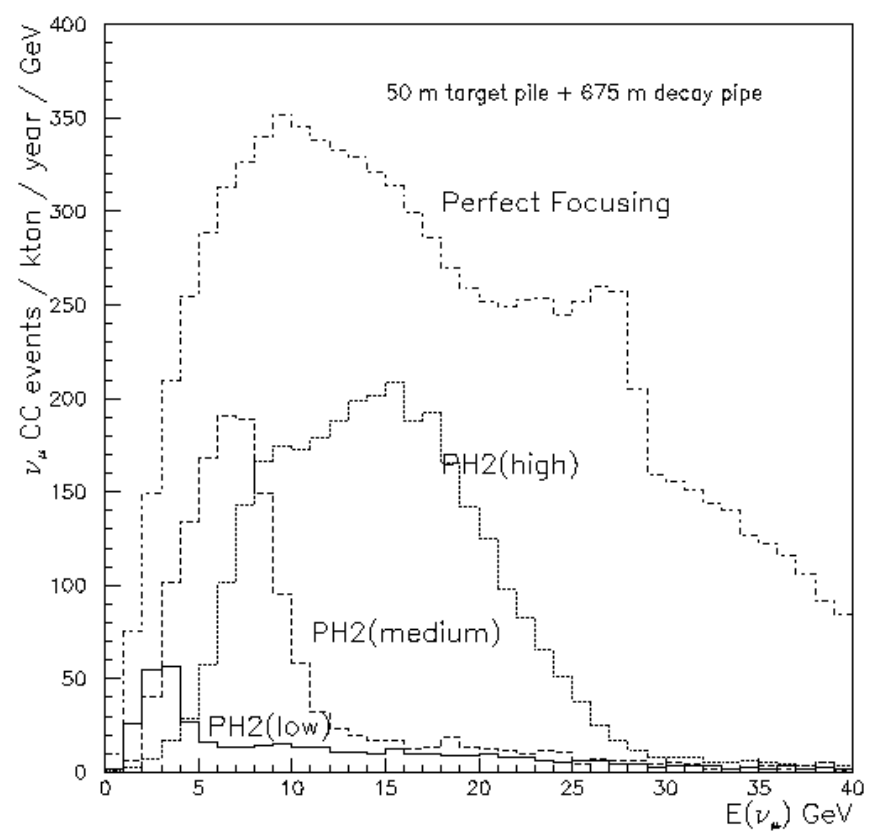

Figure 27: NuMI beam event rates at the Soudan mine location in the case of ideal focusing and of three different beam designs.

The NuMI neutrino beam is obtained by shooting $120 \mathrm{GeV}$ protons accelerated by the fast cycling Main Injector (repetition rate of $1.9 \mathrm{~s}$ ) onto a beryllium (or graphite) target $160 \mathrm{~cm}$ long. The Main Injector is expected to deliver $4 \times 10^{13}$ pot over $1 \mathrm{~ms}$. The hadrons produced in the target are focused (defocused) by two parabolic magnetic lenses and transported through an evacuated beam pipe of $1 \mathrm{~m}$ radius and $675 \mathrm{~m}$ length. The shielding of the near detector against muons is provided by a hadron absorber placed downstream of the beam pipe and by the dolomite rock between the absorber and the near detector location. The main features and the expected performance of the NuMI facility are shown in Table 9. Event rates are given in Table 10.

An additional feature of the NuMI beam is the possibility to produce neutrinos with narrow energy spectrum (NBB). The latter could be a tool for precision studies once the values of the oscillation parameters are narrowed down.

The NuMI beam simulation is based on the FLUKA model associated with the GEANT package (GEANT-FLUKA) [118]. It is common understanding that FLUKA98, which is used for the CNGS simulation, gives a pion production yield $20 \%$ lower than FLUKA-GEANT. This should be taken into account when comparing the expected performance achievable with the two beams. 


\begin{tabular}{||c|c|c|c|c||}
\hline Proton Energy & Proton/pulse & Cycle time & pot/year & $\left\langle E_{\nu}\right\rangle$ \\
\hline \hline $120 \mathrm{GeV}$ & $4 \times 10^{13}$ & $1.9 \mathrm{~s}$ & $3.6 \times 10^{20}$ & $3-16 \mathrm{GeV}$ \\
\hline \hline
\end{tabular}

Table 9: NuMI beam features and expected performance. The average energy goes from $3 \mathrm{GeV}$ for the PH2 (low) beam to $16 \mathrm{GeV}$ for the PH2 (high) beam.

\begin{tabular}{||c|c|c|c||}
\hline events $/\left(10^{19}\right.$ pot $\times$ kton $)$ & Low energy & Medium energy & High energy \\
\hline \hline$\nu_{\mu} \mathrm{CC}$ & 12.4 & 38.9 & 86.7 \\
\hline$\nu_{e} \mathrm{CC}$ & $1.5 \times 10^{-1}$ & $3.5 \times 10^{-1}$ & $4.9 \times 10^{-1}$ \\
\hline $\bar{\nu}_{\mu} \mathrm{CC}$ & 1.7 & 1.2 & $9.2 \times 10^{-1}$ \\
\hline $\bar{\nu}_{e} \mathrm{CC}$ & $3.5 \times 10^{-2}$ & $2.4 \times 10^{-2}$ & $2.4 \times 10^{-2}$ \\
\hline$\nu_{\tau} \mathrm{CC}$ at $\Delta m^{2}=0.01 \mathrm{eV}^{2}, \sin ^{2} 2 \theta_{\mu \tau}=1$ & $7.3 \times 10^{-1}$ & 3.6 & 8.4 \\
\hline$\nu_{\tau} \mathrm{CC}$ at $\Delta m^{2}=0.001 \mathrm{eV}^{2}, \sin ^{2} 2 \theta_{\mu \tau}=1$ & $1.4 \times 10^{-2}$ & $7.0 \times 10^{-2}$ & $1.1 \times 10^{-1}$ \\
\hline \hline
\end{tabular}

Table 10: Neutrino interaction rates at the Soudan mine for the three possible NuMI beams.

\subsection{The KEK-Kamioka beam}

The K2K neutrino beam $[41,119]$ from the KEK Laboratory in Japan to the Super-Kamiokande detector was commissioned in spring 1999 with the aim of a neutrino oscillation search with a long baseline experiment. The physics goal is similar to that of the CNGS and NuMI facilities. However, as described in Section 13.1, the low neutrino beam energy will not allow for a $\nu_{\tau}$ apperance search.

The beam layout is shown in Fig. 28. It is the first long baseline beam to be operational in the world and the first neutrino beam in Japan. $\nu_{\mu}$ are generated by sending with a fast extraction $12 \mathrm{GeV}$ protons from the K2K Proton Syncrotron onto an aluminium target of $2 \mathrm{~cm}$ diameter and $65 \mathrm{~cm}$ long. The time duration of one spill is $1.1 \mu \mathrm{s}$ and the frequency is one spill every $2.2 \mathrm{~s}$ with a nominal intensity of $\sim 6 \times 10^{12}$ protons/spill. A total of $10^{20}$ pot will be extracted in three years of operation.

Positively charged mesons produced in the target are focused towards Super-Kamiokande by a toroidal magnetic field produced by a pair of horns. These are followed by a $200 \mathrm{~m}$ decay tunnel. Downstream of the tunnel a beam dump absorbs the muons and those pions which did not interact in the target. The energy spectrum and the angular dependence of the beam at the near detector (300 $\mathrm{m}$ away from the target) and at the Super-Kamiokande location (distant $250 \mathrm{~km}$ ) are shown in Fig. 29.

The mean energy of the neutrino beam is $1.4 \mathrm{GeV}$ with a peak at $1 \mathrm{GeV}$. The $\nu_{e}$ contamination has been estimated to be $\sim 1 \%$. In order to determine the neutrino energy spectrum a pion monitor (a Cerenkov counter with a spherical mirror and filled with R-C318 gas) is placed downstream of the second horn. A muon monitor made of $2 \times 2 \mathrm{~m}^{2}$ pad-type ionisation chamber filled with $\mathrm{He}$ gas is installed at the end of the decay tunnel. It allows to monitor the position of the beam center with an accuracy of about $2 \mathrm{~cm}$.

\section{Neutrino oscillation experiments at CERN}

Experiments exploiting the CERN Proton Syncrotron (PS) and SPS neutrino beams have been searching for neutrino oscillations for about 30 years. For thorough reviews on early experiments we refer to [120-123]. Here we first briefly review some of the experiments which used bubble chamber detectors. We then describe 


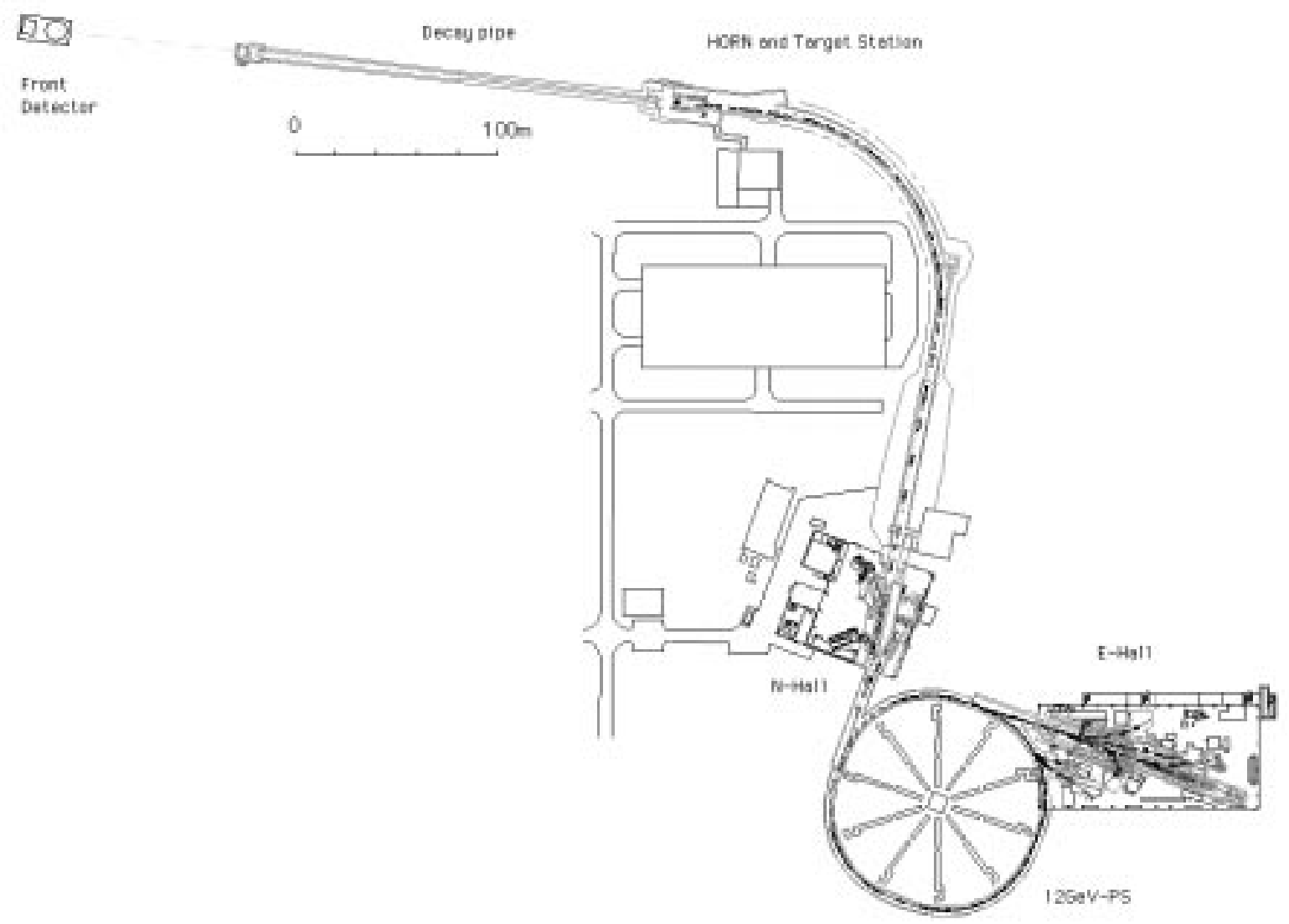

Figure 28: Layout of the K2K beam line. 

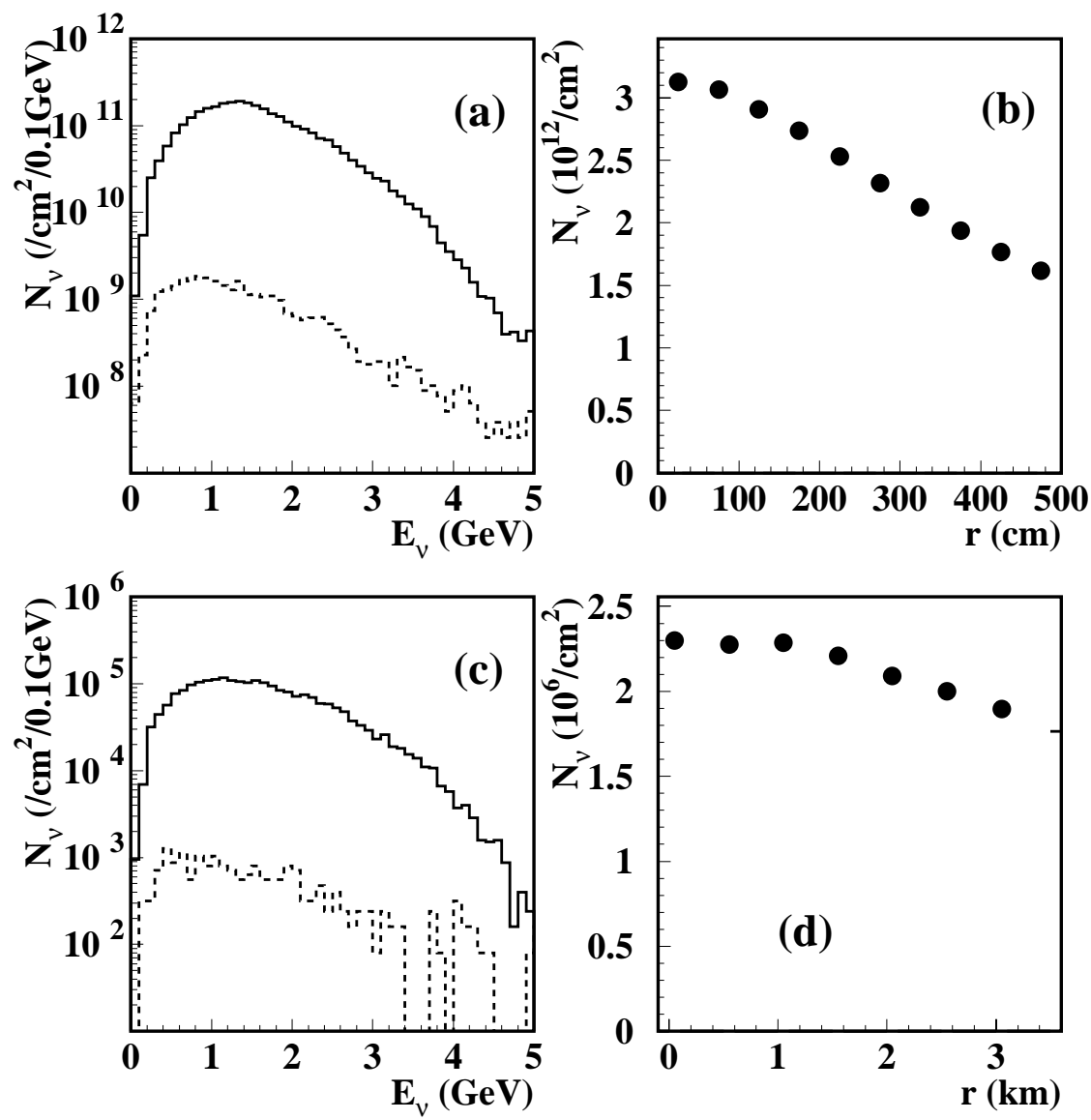

Figure 29: Energy spectrum of the K2K beam and its radial distribution for $10^{20}$ pot (corresponding to three years of operation) at the near detector location $(L=300 \mathrm{~m})$ and at the Super-Kamiokande site $(L=250 \mathrm{~km})$. 


\begin{tabular}{||c|c|c|c||}
\hline Experiment & $\begin{array}{c}\nu_{e} \leftrightarrow \nu_{\mu} \\
\Delta m^{2} \mid \sin ^{2} 2 \theta\end{array}$ & $\begin{array}{c}\nu_{\mu} \leftrightarrow \nu_{\tau} \\
\Delta m^{2} \mid \sin ^{2} 2 \theta\end{array}$ & $\begin{array}{c}\nu_{e} \leftrightarrow \nu_{e} \\
\Delta m^{2} \mid \sin ^{2} 2 \theta\end{array}$ \\
\hline \hline GGM-PS [125] & $\sim 1 \mid \sim 0.1$ & & \\
\hline GGM-PS [126] & $0.7 \mid 0.06$ & & \\
\hline BEBC-NBB [127] & & & $55 \mid 0.3$ \\
\hline BEBC-SPS [128] & $1.7 \mid 0.01$ & $6 \mid 0.05$ & $10 \mid 0.07$ \\
\hline GGM-SPS [129] & $2 \mid 0.012$ & $5 \mid 0.08$ & \\
\hline BEBC-SPS [130] & $0.7 \mid 0.001$ & & \\
\hline BEBC-PS [131] & $0.09 \mid 0.013$ & & \\
\hline \hline
\end{tabular}

Table 11: Summary of results obtained by bubble chamber experiments searching for neutrino oscillations at CERN. $\Delta m^{2}$ values are given in $e V^{2}$.

in more depth the methods and the results of more recent experiments which made use of electronic detectors.

\subsection{Bubble chamber experiments: Gargamelle and BEBC}

Neutrino oscillation experiments were performed in the seventies at CERN by using the large bubble chamber detectors Gargamelle and BEBC (Big European Bubble Chamber). The main characteristic of a bubble chamber filled with a mixture of heavy liquids $\left(\mathrm{Ne}-\mathrm{H}_{2}\right.$, freon-propane) is its high efficiency in identifying electrons. This feature allows to perform $\nu_{e}$ appearance and disappearance experiments by detecting an excess or a deficit (over the expected rate) of CC interactions induced by $\nu_{e}$.

The major limitation of those experiments is that the analysis methods rely upon the knowledge of the relative $\nu_{e} / \nu_{\mu}$ flux and hence on uncertainties on its calculation. For a detailed discussion on bubble chamber detectors we refer to [124] and references therein.

The Gargamelle and BEBC detectors exploited the PS [125,126] and SPS Wide Band Beam [105] to search mainly for $\nu_{\mu} \leftrightarrow \nu_{e}$ oscillations. A search for neutrino oscillations with a NBB was also performed with BEBC. The analysis method relies on the very good electron identification of the detector and on a detailed kinematical analysis of the events.

The occurrence of neutrino oscillations is searched for by comparing measured kinematical distributions with predictions from simulations. In Table 11 the (negative) results obtained by bubble chamber experiments are summarised ${ }^{2}$. The $90 \%$ C.L. limits on $\Delta m^{2}$ and $\sin ^{2} 2 \theta$ are listed.

\subsection{The CDHSW experiment}

The CDHSW [132] and CHARM [133] experiments searched for neutrino oscillations using a beam from the CERN Proton Syncrotron (PS). This beam, especially dedicated to neutrino oscillation searches, was set up in 1982 [133] and made use of $19.2 \mathrm{GeV}$ protons (Fig. 30).

On average $1.2 \times 10^{13}$ protons with a repetition rate of $1.2 \mathrm{~s}$ are sent onto a cylindrical beryllium target

\footnotetext{
${ }^{2}$ Oscillation experiments using bubble chambers were also conducted at Serpukhov. In particular, we mention the $\nu_{\mu} \leftrightarrow \nu_{e}$ oscillation search performed with the SKAT heavy liquid bubble chamber [161]. The detector was exposed to a WBB with neutrino energies between 3 and $30 \mathrm{GeV}$ [161]. The oscillation search proceeded by studying the ratio between $\nu_{e} \mathrm{CC}$ and $\nu_{\mu} \mathrm{CC}$ interactions. No oscillation signal was found. $\Delta \mathrm{m}^{2}$ values larger than $1.3 \mathrm{eV}$ are excluded at the $90 \%$ C.L. for full mixing. $\sin ^{2} 2 \theta$ larger than $2.5 \times 10^{-3}$ are excluded for $\Delta m^{2}=60 \mathrm{eV}^{2}$.
} 


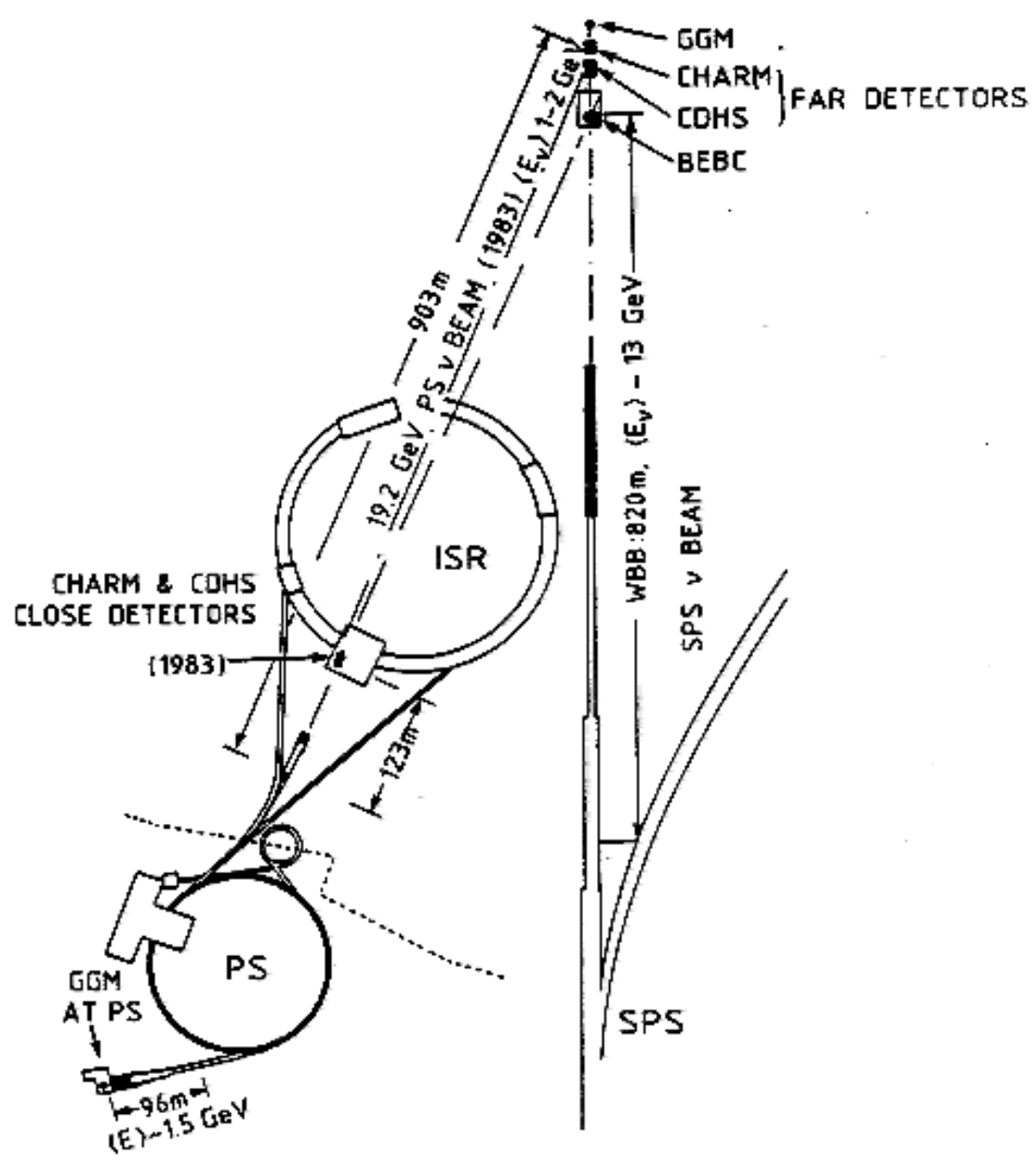

Figure 30: The CERN proton-synchrotron (PS) neutrino beam line dedicated to neutrino oscillation searches. 
$60 \mathrm{~cm}$ long and $8 \mathrm{~mm}$ in diameter. Most of the daughter pions and kaons decay in a $48 \mathrm{~m}$ long, $2.8 \mathrm{~m}$ high and $5 \mathrm{~m}$ wide decay tunnel filled with air at atmospheric pressure. Mostly $\nu_{\mu}$ and $\bar{\nu}_{\mu}$ are produced. The decay tunnel is followed by a $50 \mathrm{~cm}$ concrete wall, a $4 \mathrm{~m}$ long iron absorber and $50 \mathrm{~m}$ of molasse. The mean energy of the neutrino beam is about $1 \mathrm{GeV}$.

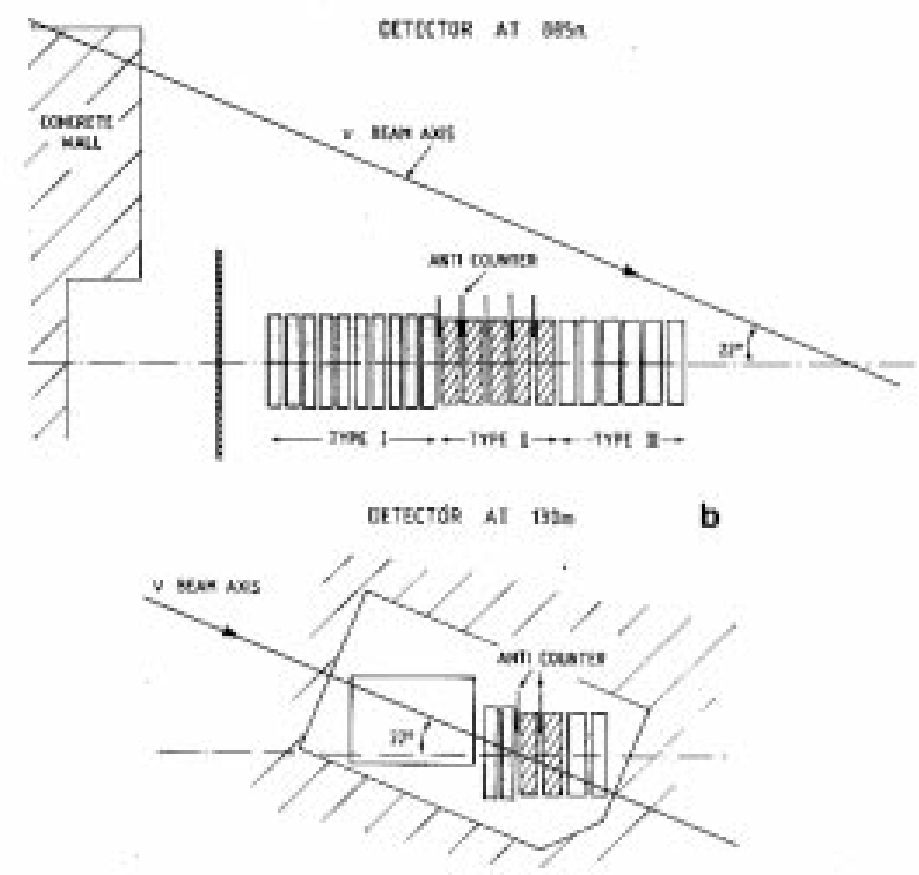

Figure 31: Layout of the near and far CDHSW detectors.

The main purpose of the CDHSW experiment [132] was the study of neutrino interaction properties exploiting the CERN Super Proton Syncrotron (SPS) WANF neutrino beam. For the study of $\nu_{\mu} \leftrightarrow \nu_{x}$ oscillations with small $\Delta m^{2}$ the PS neutrino beam was pointed towards the CDHSW detector $885 \mathrm{~m}$ far from the beryllium target. A second (near) detector identical in design to the main (far) setup was placed $130 \mathrm{~m}$ away from the proton target to remove the need for the absolute flux normalisation.

Both detectors consist of three distinct types of modules with different granularity made of sandwiches of circular iron plates and plastic scintillator planes both with $3.75 \mathrm{~m}$ diameter (Fig. 31). The far (near) detector has a total mass of 600 (100) ton.

The trigger of the experiment is given by a signal measuring the thickness of iron traversed by the most penetrating particle in the event. The minimal length for an event required to issue a trigger corresponds to about $30 \mathrm{~cm}$ of iron.

Cuts on the fiducial volume and on the direction of the total momentum of the events are applied to eliminate the background induced by cosmic rays and by neutrino interactions outside the detector. The total reconstructed momentum vector is required to have a vertical angle of less than $45^{\circ}$ with respect to the axis of the detector and not to enter from the top of the detector.

Triggered events are classified according to their range in iron projected onto the detector axis. This requirement allows not to be sensitive to the pulse height calibration of the two setups. A minimal projected 
range of $40 \mathrm{~cm}$ is required in order to have full trigger efficiency for both the near and far detectors. The mean energy of neutrinos accepted by this cut amounts to about $3 \mathrm{GeV}$. The cut also reduces the contamination of $\mathrm{NC}$ events to less than 5\%. The final data sample contains $\sim 22000$ events in the near detector with a contamination of 50 cosmic-ray induced events and $\sim 3300$ events in the far detector contaminated by 290 cosmic-ray events.

In an ideal experiment the near/far ratio is expected to be one in the absence of oscillations once corrected for the different detector masses and the reduction of the neutrino flux. In real life a Monte Carlo simulation is needed to take into account different geometries and performance of the two detectors and the differences in the neutrino spectrum within the covered solid angle. For this reason the double ratio is

quoted $R=\left(N_{\text {far }} / N_{\text {near }}\right)^{\text {observed }} /\left(N_{\text {far }} / N_{\text {near }}\right)^{M C}$. This quantity is found to be $1.044 \pm 0.023$. The expected value of $R$ in the case of oscillations with small $\Delta m^{2}$ is well below the unity.

The most serious sources of systematic errors are: dead time corrections, differences in the event reconstruction in the two detectors, contamination from events produced outside and not identified as such, uncertainty on the knowledge of the neutrino beam composition, uncertainties on the neutrino cross-sections and on the subtraction of cosmic-ray induced events. The overall systematic error is estimated to be $2.5 \%$.

The $90 \%$ C.L. limit of the CDHSW experiment for the $\nu_{\mu} \leftrightarrow \nu_{x}$ oscillation search is shown in Fig. 32 . The lack of sensitivity for $\Delta m^{2}>100 \mathrm{eV}^{2}$ is related to the normalisation method, which is based on the use of a near detector.

\subsection{The CHARM experiment}

The CHARM experiment [133] was designed to study neutrino interactions using the WANF beam. The detector is shown in Fig. 33. It is made of a fine-grained calorimeter and of a muon spectrometer. The target calorimeter contains 78 modules each of them made of a plane of 20 scintillator counters, a layer of streamer tubes, a marble plate $\left(300 \times 300 \times 8 \mathrm{~cm}^{3}\right)$ and a layer of proportional drift tubes. A $45 \mathrm{~cm}$ wide magnetised-iron frame surrounds each module.

The main characteristics of the detector are its high efficiency in the detection of showers down to low energies $\left(\varepsilon>99 \%\right.$ for $\left.E_{s h} \geq 1.5 \mathrm{GeV}\right)$, the good hadronic energy resolution $\sigma\left(E_{h}\right) / E_{h}=0.47 / \sqrt{E(G e V)}$, the pattern recognition capabilities allowing the detection of muons with momenta as low as $1 \mathrm{GeV}$ and the electromagnetic shower identification down to $0.5 \mathrm{GeV}$. These features allow to search for $\nu_{\mu} \leftrightarrow \nu_{e}$ oscillations in appearance mode.

The CHARM detector was also exposed to the CERN-PS neutrino beam. 18 modules of the calorimeter (27 ton total mass) were moved close to the neutrino source to form the near detector (123 $\mathrm{m}$ far from the proton target). 20 magnetised iron plates equipped with proportional drift tubes were installed behind the calorimeter in order to enhance the detection efficiency for long muon tracks (muon catcher). The remaining 60 modules of the CHARM calorimeter, together with the muon spectrometer, compose the far detector placed $903 \mathrm{~m}$ far from the proton target for a total mass of 122 ton. In the following, two independent searches for oscillations performed with the CHARM detector $\left(\nu_{\mu}\right.$ disappearance and $\nu_{\mu} \leftrightarrow \nu_{e}$ appearance) are presented. 


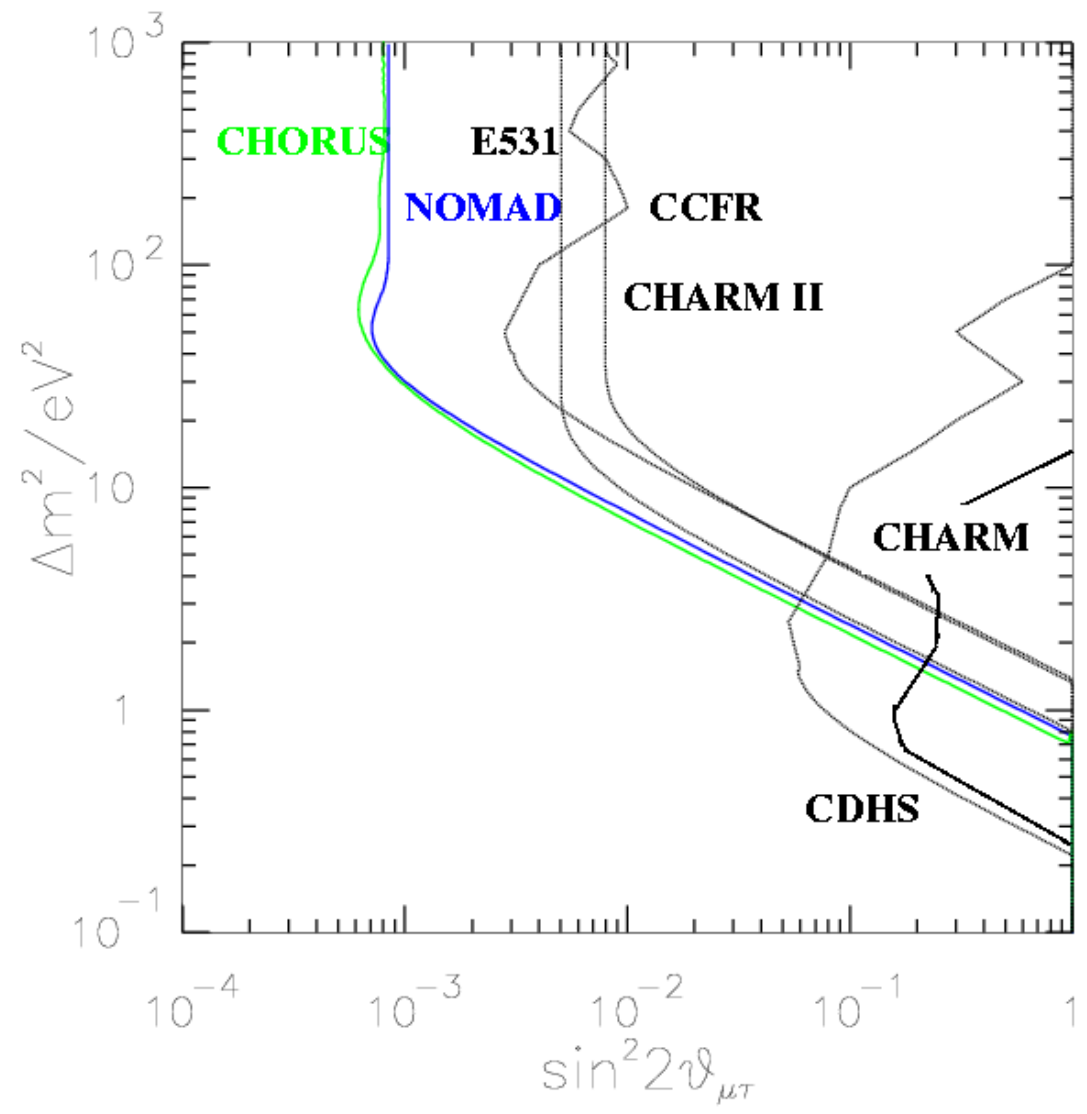

Figure 32: Exclusion plots obtained by different experiments searching for $\nu_{\mu} \leftrightarrow \nu_{\tau}$ oscillations. 


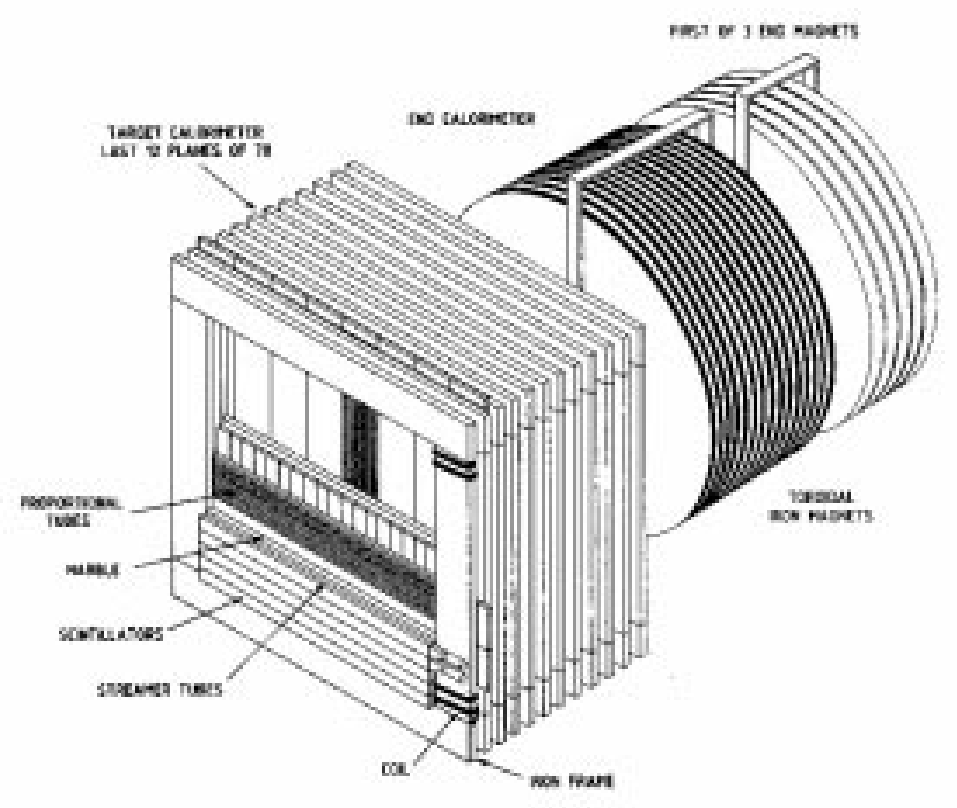

Figure 33: Schematic view of the CHARM detector.

\subsection{1 $\nu_{\mu}$ disappearance search}

The presence of a near and a far detector allowed a $\nu_{\mu}$ disappearance search [133] similar to the CDHSW experiment. An analysis algorithm was developed selecting quasi-elastic (QE) interactions in order to reject $\mathrm{NC}$ interactions and to enhance the detection of $\mathrm{CC}$ reactions. A pre-selection of the events is basically performed by requiring low hit multiplicity in consecutive scintillator planes, compatible with the signature of a muon track. For events satisfying this criterion a track fit is tried and those that survive the following cuts are retained

- successful track fit;

- fits in projections matching longitudinally;

- track angle smaller than $53^{\circ}$ with respect to the detector axis. This cut rejects most of the events with tracks entering or leaving the detector from sides, top or bottom;

- fitted track contained in the detector. This requirement allows to reject backward going cosmic rays and to determine the muon momentum by range.

Each event is characterised by the projected length $l$ measured by the scintillators and expressed in the number of traversed detector planes. According to the value of $l$, two event samples are defined

$$
4 \leq l \leq 15 \text { and } l>15
$$


In order to compare the rates in the two detectors, each event belonging to the sample with $4 \leq l \leq 15$ is weighted by the inverse of the probability that an event of projected length $l$ could be detected in the available number of planes. The number of events belonging to this sample is 2043 in the near detector and 268 in the far. The ratio of the weighted events $r_{o b s}$ corrected for backgrounds and inefficiencies amounts to

$$
r_{\text {obs }}=\frac{N_{F D}}{N_{N D}} \times c_{\text {dead }} \times c_{\text {cosmic }} \times c_{\text {double }}=0.0171 \pm 0.0010 \pm 0.0009
$$

where $N_{F D}$ and $N_{N D}$ are the weighted numbers of events in the far and near detector, respectively, $c_{\text {dead }}$ is the dead time correction factor, $c_{\text {cosmic }}$ is a factor related to the cosmic-ray subtraction and $c_{\text {double }}$ takes into account the probability for double events in the same acquisition gate.

The expected ratio $r_{\text {exp }}$ in the absence of oscillations is estimated by using a Monte Carlo simulation to be $0.0178 \pm 0.0003$, where the error is only systematical. The ratio of observed to expected events is then $r_{\text {obs }} / r_{\text {exp }}=0.96 \pm 0.06 \pm 0.05$.

Events with projected length $l$ larger than 15 planes are analysed separately as they need a different treatment in the near and far detectors. The statistical significance of this sample is lower due to the limited statistics: 1329 and 45 events are detected in the far and near detector, respectively. The ratio of observed to expected events is in this case $r_{\text {obs }} / r_{\text {exp }}=1.21 \pm 0.20 \pm 0.04$. Both samples are consistent with the absence of neutrino oscillations. This corresponds to the $90 \%$ C.L. best limit of $\sin ^{2} 2 \theta<0.17$ and to $\Delta m^{2}<0.29 \mathrm{eV}^{2}$ for full mixing.

\subsection{2 $\nu_{e}$ appearance search}

The excellent capability of the CHARM detector in identifying electromagnetic showers allowed an appearance search for $\nu_{\mu} \leftrightarrow \nu_{e}$ oscillations [133] to be performed. Two independent searches for $\nu_{e}$ interactions were accomplished by using events with projected length smaller than 15 planes. A first search was performed by eye-scanning the whole set of interactions in the far and near detectors. 66 (19) electron candidates were selected in the near (far) detector with an average energy of $1.65 \mathrm{GeV}$.

After applying corrections for efficiencies the $\nu_{e} / \nu_{\mu} \mathrm{CC}$ interaction ratios for the two distances are

$$
r_{N}=(9.1 \pm 1.1 \pm 1.1) \% \quad r_{F}=(8.9 \pm 2.0 \pm 1.0) \%
$$

where the first error is statistical and the second systematic. Once known backgrounds have been subtracted and the response of the near and far detectors to NC events has been assumed to be the same, less than $2.7 \%$ (90\% C.L.) of the $\nu_{e}$ are estimated to originate from $\nu_{\mu}$ oscillations.

A second search was done by using a computer algorithm for the identification of $\nu_{e}$ induced events. The values for $r_{F}$ and $r_{N}$ are then

$$
r_{N}=(-0.5 \pm 1.1) \% \quad r_{F}=\left(-3.1_{-2.2}^{+4.8}\right) \%
$$

For small $\Delta m^{2}$ values oscillations could only be seen in the far detector. Therefore, the near detector can be used to subtract the background. Assuming equal systematic errors in both detectors the value of the quantity $r_{N}-r_{F}$ turns out to be compatible with an oscillation probability $P\left(\nu_{\mu} \leftrightarrow \nu_{e}\right) \leq 3.7 \times 10^{-2}$ the $90 \%$ C.L. 
A similar experiment was performed in the SPS neutrino beam [133]. $\Delta m^{2}$ values larger than $0.19 \mathrm{eV}^{2}$ are excluded at the $90 \%$ C.L. for full mixing. $\sin ^{2} 2 \theta$ larger than $8 \times 10^{-3}$ are excluded for $\Delta m^{2}>30 e V^{2}$.

\subsection{The CHARM II experiment}

The principal aim of the CHARM II experiment $[134,135]$ has been a high statistics study of the reaction

$$
\nu_{\mu}\left(\bar{\nu}_{\mu}\right)+e^{-} \rightarrow \nu_{\mu}\left(\bar{\nu}_{\mu}\right)+e^{-}
$$

for the determination of the Standard Model parameters in the leptonic sector. The detector consists of a massive, low-density, fine-grained calorimeter (700 ton in weight and $35 \mathrm{~m}$ long) and of a muon spectrometer. The basic building element of the calorimeter is a module made of a $4.8 \mathrm{~cm}$ thick glass target $\left(0.5 X_{0}\right.$ or $1 / 9 \lambda_{\text {int }}$ ) followed by a plane of plastic streamer tubes with $1 \mathrm{~cm}^{2}$ cross-section read out in digital and analog mode. The total number of modules is 420 . Behind each group of five modules a plane of $3 \mathrm{~cm}$ thick, $15 \mathrm{~cm}$ wide and $3 \mathrm{~m}$ long plastic scintillator counters is inserted to measure $d E / d x$ for $e / \pi^{0}$ discrimination.

The low $Z$ material and the granularity of the calorimeter ensures the required angular resolution for electron showers. The electromagnetic energy resolution achieved by using the digital signal of the streamer tubes is $\sigma(E) / E=0.15 / \sqrt{E(G e V)}+0.09$. The spectrometer consists of magnetised iron toroids interleaved with scintillator counters and drift chambers. It measures the muon momentum with a resolution $\Delta p / p=13 \%$ at $20 \mathrm{GeV}$. The layout of the detector is shown in Fig. 34. A special dimuon event is shown in Fig. 35 to illustrate the fine calorimeter granularity.

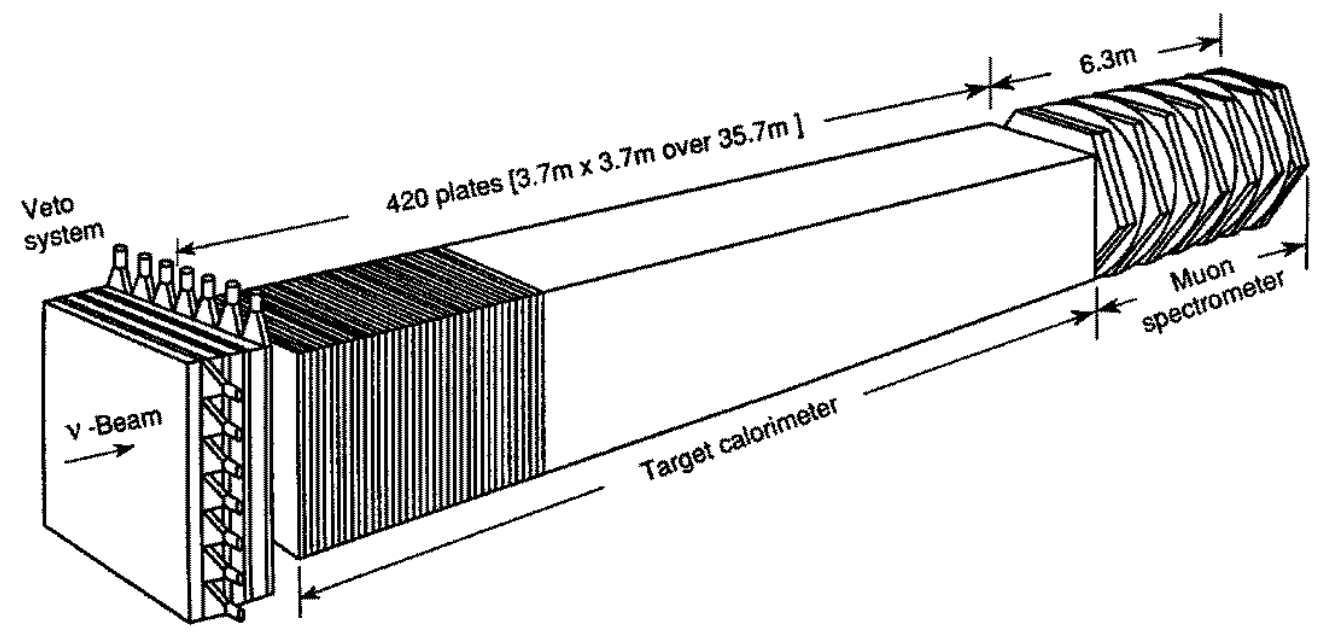

Figure 34: Schematic view of the CHARM II detector.

\subsection{1 $\nu_{\mu} \leftrightarrow \nu_{e}$ oscillation search}

A $\nu_{\mu} \leftrightarrow \nu_{e}$ oscillation search was performed with the CHARM II detector [136]. The data used in that analysis were collected in the 1987 - 1991 running period by exposing the detector to the WANF beam for a total of $2.6 \times 10^{19}$ pot. The main component of the beam could be either $\bar{\nu}_{\mu}$ or $\nu_{\mu}$ according to the polarity of horn and reflector. The integrated flux ratio between the $\bar{\nu}_{\mu}$ and the $\nu_{\mu}$ exposures is 1.2. The 


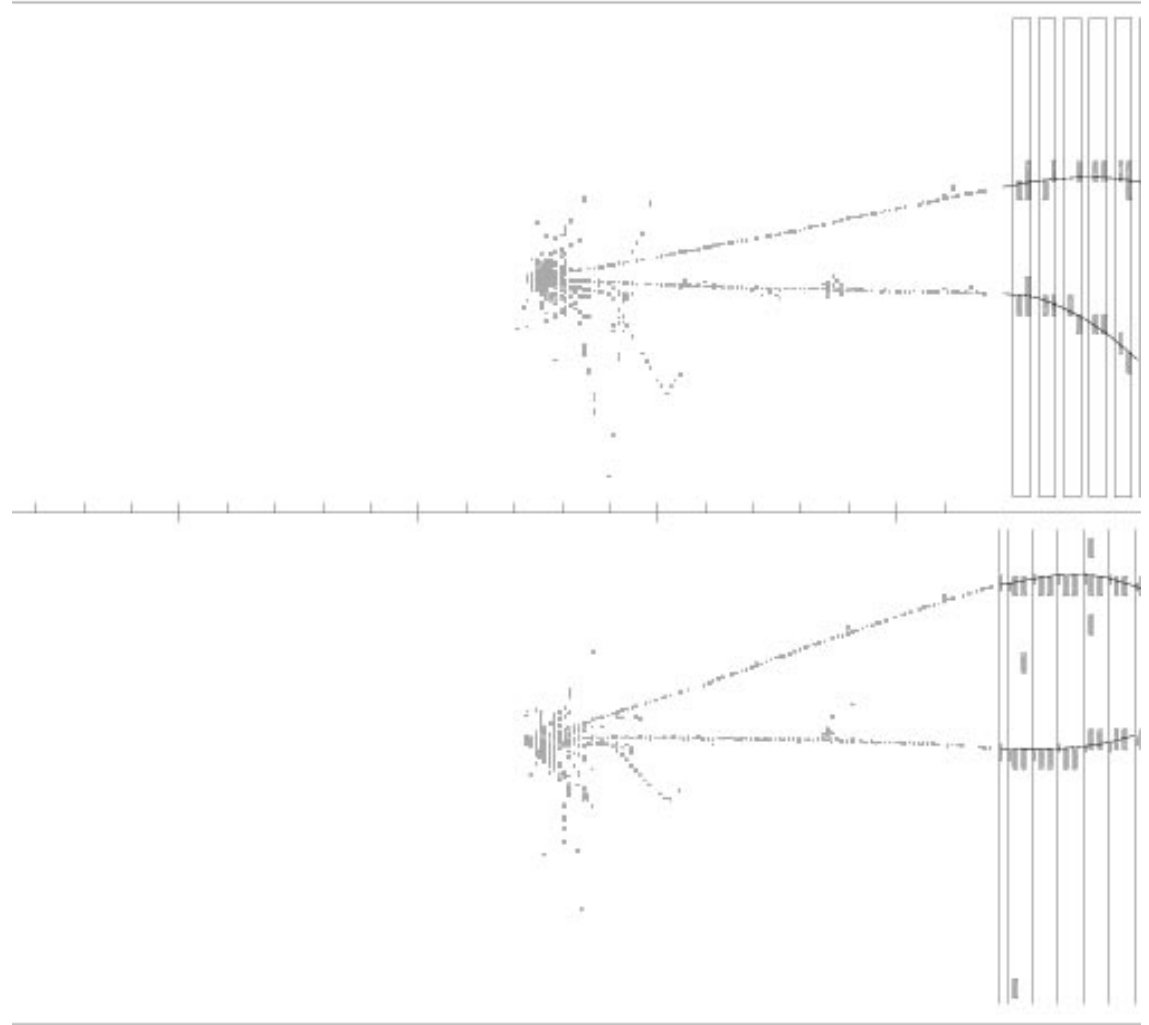

Figure 35: Display of a dimuon event in the CHARM II calorimeter. The two views refer to the horizontal and vertical projections. 


\begin{tabular}{||c|c|c||}
\hline$E_{\text {shower }}$ & $5-20 \mathrm{GeV}$ & $20-35 \mathrm{GeV}$ \\
\hline \hline e.m. showers & $2641(3208)$ & $673(766)$ \\
\hline$N_{e}^{\text {obs }}$ & $252 \pm 59(402 \pm 68)$ & $158 \pm 27(277 \pm 33)$ \\
\hline$N_{e}^{e x p}$ & $149 \pm 27(300 \pm 53)$ & $122 \pm 22(253 \pm 46)$ \\
\hline$N_{e}^{\text {exc }}$ & $103 \pm 65(102 \pm 86)$ & $36 \pm 34(24 \pm 56)$ \\
\hline \hline
\end{tabular}

Table 12: Result of the search for $\nu_{\mu}\left(\bar{\nu}_{\mu}\right) \leftrightarrow \nu_{e}\left(\bar{\nu}_{e}\right)$ oscillations with the CHARM II detector using low and high energy events.

starting data sample contains events with low detector activity at the interaction vertex. They originate from quasi-elastic interactions

$$
\nu_{l} n \rightarrow l^{-} p \text { and } \bar{\nu}_{l} p \rightarrow l^{+} n
$$

with $l=e, \mu$.

These events fulfil the electron trigger $[134,135]$ and further criteria to distinguish electromagnetic from hadronic showers. Only events whose vertex occurs in a module followed by a scintillator plane are accepted since the energy deposited in the scintillators plays a crucial role in the analysis. The sample of electromagnetic showers contains four different categories of events

- QE interactions of $\nu_{e}$ contaminating the beam;

- $\nu_{\mu} e \rightarrow \nu_{\mu} e$ electromagnetic induced showers;

- single $\pi^{0}$ production in $\nu_{\mu}$ and $\bar{\nu}_{\mu} \mathrm{NC}$ interactions;

- possible $\nu_{e} \mathrm{QE}$ interactions originated from $\nu_{\mu} \leftrightarrow \nu_{e}$ oscillations.

The subtraction of the $\nu_{\mu} e \rightarrow \nu_{\mu} e$ events is relatively straightforward being the study of this reaction the main aim of the CHARM II experiment [137]. The rejection of single $\pi^{0} \mathrm{~s}$ is performed by looking at the signal of the first scintillator plane following the vertex $\left(E_{\text {first }}\right)$. By requiring $E_{\text {first }}<8 \mathrm{MeV}$ $89.8 \pm 4.4$ (stat.) \pm 6.5 (syst.) $\%$ of the single $\pi^{0}$ events are rejected. $56 \pm 1.5($ stat. $) \pm 1.6$ (syst. $) \%$ of the $\nu_{e}$ QE interactions are accepted for the $\nu_{\mu}$ exposure and $58 \pm 1.4$ (stat.) \pm 2.3 (syst.) $\%$ for the $\bar{\nu}_{\mu}$ exposure. Two energy intervals are considered: $5 \leq E_{\text {shower }} \leq 20 \mathrm{GeV}$ and $20 \leq E_{\text {shower }} \leq 35 \mathrm{GeV}$ since the relative contribution of the background processes depends on the energy. The selected event samples of electromagnetic showers before and after the cuts are shown in Table 12 for both neutrino and anti-neutrino runs.

The expected number of events $N_{e}^{e x p}$ is determined by a Monte Carlo simulation of the neutrino beams and of the detector response to neutrino interactions. The results are shown in Table 12 together with the excess of observed events $\left(N_{e}^{e x c}=N_{e}^{o b s}-N_{e}^{e x p}\right)$. No evidence for $\nu_{\mu} \leftrightarrow \nu_{e}$ oscillations is found being $N_{e}^{e x c}$ consistent with zero for both regions. This negative result leads to the following $90 \%$ C.L. limits obtained using both energy intervals

$$
P\left(\nu_{\mu} \leftrightarrow \nu_{e}\right)<4.7 \times 10^{-3} \quad P\left(\bar{\nu}_{\mu} \leftrightarrow \bar{\nu}_{e}\right)<2.4 \times 10^{-3}
$$


The above measurements may be combined under the assumption of CP invariance. A total excess of $265 \pm 178$ events is derived. It corresponds to the $90 \%$ C.L. limit

$$
P\left(\nu_{\mu}\left(\bar{\nu}_{\mu}\right) \leftrightarrow \nu_{e}\left(\bar{\nu}_{e}\right)\right)<2.8 \times 10^{-3}
$$

\subsection{2 $\nu_{\mu} \leftrightarrow \nu_{\tau}$ oscillation search}

The CHARM II experiment also searched for $\nu_{\mu} \leftrightarrow \nu_{\tau}$ oscillations [138]. We recall that each target plane of the calorimeter is $1 / 9 \lambda_{\text {int }}$ thick. A high energy hadron has then a finite probability to travel through several planes before interacting. $\nu_{\tau} N \rightarrow \tau N^{\prime}$ QE interactions with subsequent $\tau \rightarrow h \nu_{\tau}$ decay could then be detected. The signature of these events is a minimum ionising track followed by a hadronic shower whose axis coincides with the track direction.

The background to the above channel originates from $\nu_{\mu} N \rightarrow \nu_{\mu} h X$ interactions in which a singlecharged hadron of high energy is accompanied by one or more particles (the system $X$ ) with an energy too low to produce a visible signal in the detector. The background from the possible $\nu_{\tau}$ contamination of the beam is negligible.

The data used for that analysis were collected in a run of about 100 days with a total of $5 \times 10^{18}$ pot in the ratio $1 / 1.4$ between $\nu_{\mu}$ and $\bar{\nu}_{\mu}$ fluxes. The trigger selects $3 \times 10^{6}$ events reduced to 71000 after a filter to reject cosmic-ray events is applied.

Algorithms are used to identify events with a track followed by a shower. The main selection criteria consist of the logic AND of the following cuts

- the track length has to be longer than 15 planes from the vertex up to the starting point of the shower;

- the shower energy has to be higher than $10 \mathrm{GeV}$;

- the number of hits in excess to those attributed to the incoming track counted in the first 8 planes of the events has to be at least 3 ;

- the amplitude of the streamer-tube analog-signal produced by the track is required to be lower than the peak value expected for a single particle increased by 1.5 standard deviations. In this way, events with two nearly parallel tracks travelling at a distance smaller than the streamer tube dimensions are rejected.

The final sample fulfilling the above cuts includes 124 events, 77 collected in the $\nu_{\mu}$ beam and 47 in the $\bar{\nu}_{\mu}$ beam. The neutrino oscillation hypothesis is tested by comparing the data with Monte Carlo simulated events: $\nu_{\mu} N \rightarrow \nu_{\mu} h X$ and $\nu_{\tau} N \rightarrow \tau N^{\prime}$ (followed by the decay $\tau \rightarrow \nu_{\tau} h$ ). The quality of the fits without any contribution from $\nu_{\tau}$ events is satisfactory. This leads to the following $90 \%$ C.L. limits

$$
\begin{gathered}
\sin ^{2} 2 \theta_{\mu \tau}<8 \times 10^{-3}\left(\text { large } \Delta m^{2}\right) \\
\Delta m_{\mu \tau}^{2}<1.5 e V^{2}\left(\sin ^{2} 2 \theta_{\mu \tau}=1\right)
\end{gathered}
$$

The exclusion plot obtained by the CHARM II experiment is shown in Fig. 32. 


\subsection{The CHORUS experiment}

CHORUS $[35,139]$ was proposed together with the NOMAD experiment (discussed in Section 7.6) in the early nineties to search for $\nu_{\mu} \leftrightarrow \nu_{\tau}$ neutrino oscillations in the parameter domain corresponding to a $\nu_{\tau}$ as a possible Dark Matter candidate [140-143].

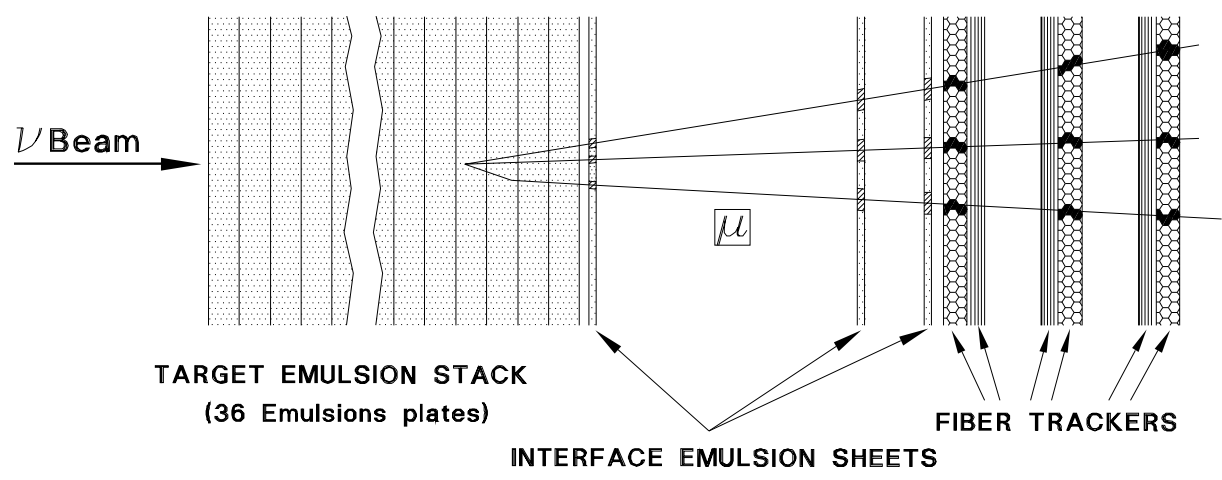

Figure 36: Schematics of a $\nu_{\tau} N \rightarrow \tau^{-} X$ interaction and subsequent $\tau$ decay in a CHORUS emulsion target stack.

In CHORUS, similar to the former E531 experiment (Section 9.2), the signature of $\nu_{\mu} \leftrightarrow \nu_{\tau}$ oscillations is the explicit detection of the $\tau$ produced in the $\nu_{\tau} N \rightarrow \tau^{-} X$ CC interaction and of its subsequent decay (kink for one-prong decays and star for three-prong decays), as schematically shown in Fig. 36. All decay channels of the $\tau^{-}$can be studied

$$
\tau^{-} \rightarrow e^{-} \overline{\nu_{e}} \nu_{\tau} ; \quad \tau^{-} \rightarrow \mu^{-} \overline{\nu_{\mu}} \nu_{\tau} ; \quad \tau^{-} \rightarrow h^{-}\left(n \pi^{0}\right) \nu_{\tau} ; \quad \tau^{-} \rightarrow \pi^{+} \pi^{-} \pi^{-} n \pi^{0} \nu_{\tau}
$$

with branching ratios of about $18 \%, 18 \%, 50 \%$ and $14 \%$, respectively.

At the typical energies of the CERN WANF beam the $\tau$ leptons produced by $\nu_{\tau}$ originating from oscillations with $\Delta \mathrm{m}^{2} \sim 10-100 \mathrm{eV}^{2}$ have an average flight path of the order of $1 \mathrm{~mm}$ (the mean life of the $\tau$ is $3 \times 10^{-13} \mathrm{~s}$ ). The smallness of the decay length motivates the need for a detector with very high space granularity and resolution, in which the $\nu_{\tau}$ could interact and produce a $\tau$ that can be detected before its decay. Nuclear emulsions fulfil these requirements.

CHORUS adopts the hybrid approach of combining nuclear emulsions and electronic detectors. The experimental setup [107] is depicted in Fig. 37. In its final configuration it is composed of an emulsion target, a scintillating fiber tracker, scintillator trigger hodoscopes, an air-core magnet equipped with emulsion and fiber trackers, a honeycomb tube detector, a lead-scintillator calorimeter and a muon spectrometer. The electronic detectors have the twofold task of locating neutrino interactions in the emulsion and performing the kinematical analysis of the events. 


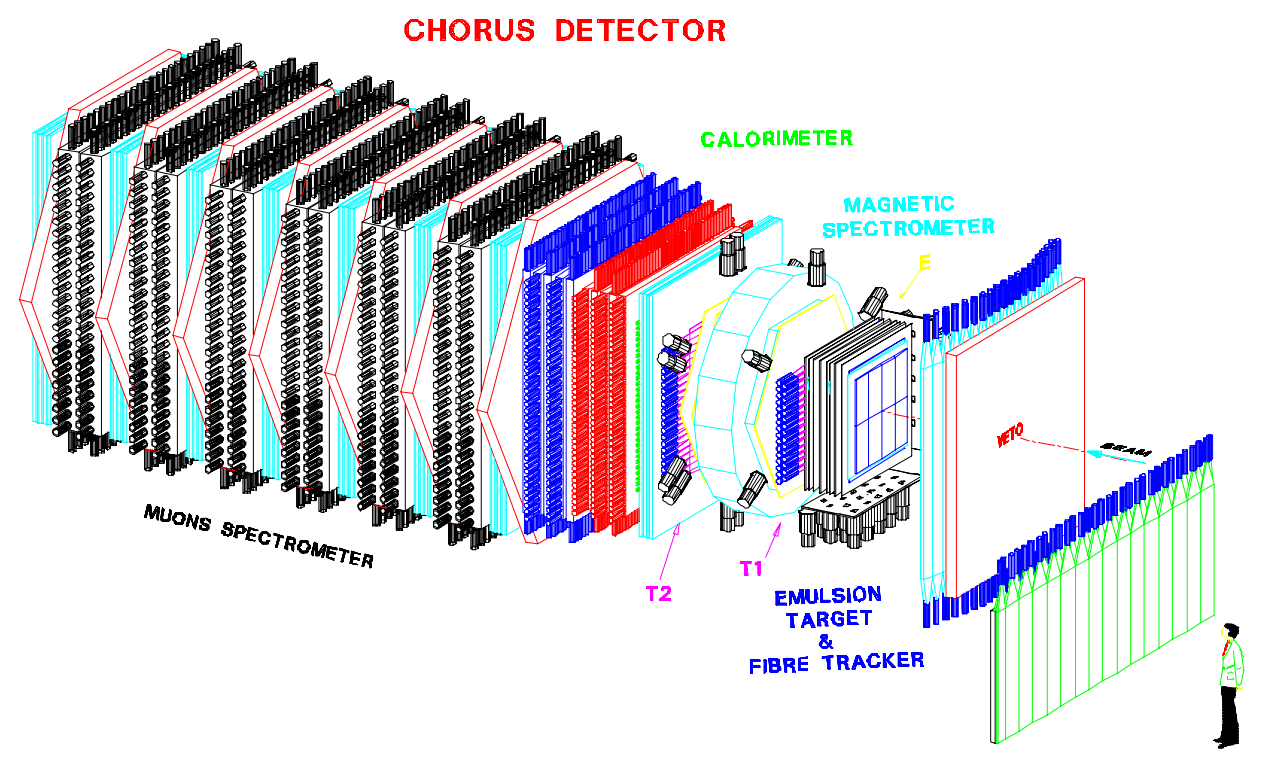

Figure 37: Schematic view of the CHORUS detector.

\subsubsection{The detector}

The target consists of $770 \mathrm{~kg}$ of nuclear emulsions. It is subdivided into four stacks each having a surface area of $1.42 \times 1.44 \mathrm{~m}^{2}$ and a thickness of $2.8 \mathrm{~cm}$. Each stack is further subdivided into 8 sectors of $0.71 \times 0.36 \mathrm{~m}^{2}$ area each consisting of a sandwich of 36 emulsion sheets. For each sheet emulsion gel with a thickness of $350 \mu \mathrm{m}$ is poured onto both faces of a $90 \mu \mathrm{m}$ plastic foil.

Emulsions provide three-dimensional space information with an excellent resolution better than $1 \mu \mathrm{m}$ as well as a high hit density of $300 \mathrm{hits} / \mathrm{mm}$ for a minimum ionising track. They are therefore ideal for the unambiguous detection of short-lived particles. Emulsion stacks are replaced every two years of data taking. Reconstruction and analysis of the neutrino events stored in the emulsion target is carried out by automatic scanning systems.

The electronic detectors are placed downstream of the emulsion target. The fiber tracker system provides accurate trajectory predictions back to the emulsions. Its position resolution is $180 \mu \mathrm{m}$ and the angular resolution $2 \mathrm{mrad}$. Scintillating fibers have the task to provide time resolution to the emulsions, correlating the occurrence of a given event with its vertex position in the emulsions.

Together with the air-core magnet, the fiber tracker allows to measure charge and momentum of the hadrons and of low momentum muons. Additional momentum measurement for selected events can be obtained with the emulsion tracker made of emulsion planes installed in front and behind the magnet. The momentum resolution of the magnet is $\Delta p / p=3.5 \% p(\mathrm{GeV})+22 \%$.

The calorimeter provides high-resolution measurement of the energy and direction of the hadronic $(\sigma(E) / E=(32.3 \pm 2.4) \% / \sqrt{E(G e V)}+(1.4 \pm 0.7) \%)$ and electromagnetic $(\sigma(E) / E=(13.8 \pm$ $0.9) \% / \sqrt{E(G e V)}+(-0.2 \pm 0.4) \%)$ showers as well as muon tracking capabilities. The charge and the momentum of the muons are measured by the muon spectrometer with a momentum resolution of about 
$20 \%$ at $70 \mathrm{GeV}$.

The trajectories of charged particles reconstructed by the fiber tracker are traced back into thin interface emulsion-plates. These are placed immediately downstream of the emulsion target where scanning starts. Once track segments are found in the interface plates one can then extrapolate them with high accuracy into the emulsion target for the location of the interaction vertex position (Section 9.2).

The scanning procedure in CHORUS benefits from the impressive progress in the field of the automatic emulsion scanning. This technique has been pioneered by the Nagoya group [144]. They have recently delivered a third generation automatic system about 1000 times faster than previous semi-automatic systems [145]. Intense R\&D programmes are also underway in Europe and Japan [145-148] with the aim to cope with the requirements of CHORUS and of other future (even more demanding) experiments (Section 13.4). Fig. 38 depicts the principle of operation of an automatic microscope system for track reconstruction.

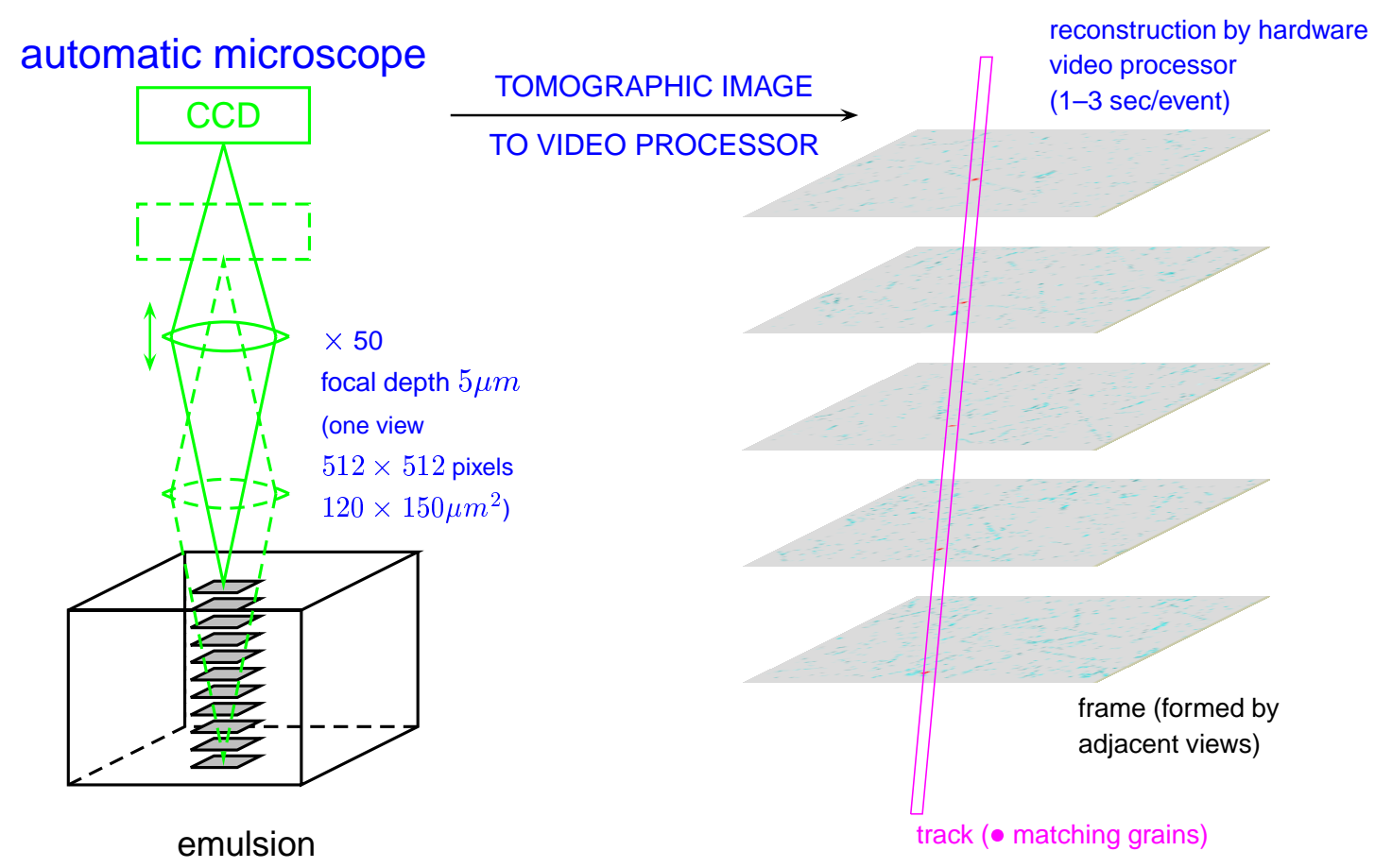

Figure 38: Working principle of an automatic microscope system: the Nagoya Track Selector. Microscope views are taken at various emulsion focal depths. The tomographic image is then sent to a processor which associates matching grains in different slices according to a predicted track angle.

Once typical decay topologies are located in the emulsion target, kinematical analysis and particle identification provide means to discriminate a possible $\tau^{-}$signal from background events with similar topologies, such as those due to charm decays or to the elastic scattering of pions.

CHORUS collected over two million triggers in its running period between 1994 and 1997 for a total of $5 \times 10^{19}$ pot (Table 13$) .460000$ events have a muon identified in the final state $(1 \mu$ events) with a vertex position compatible with one of the four emulsion target stacks. 120000 muonless events are classified as 
$0 \mu$ events. Fig. 39 shows an event taken during the neutrino run with the WANF beam. The event is due to a $\nu_{\mu} \mathrm{CC}$ interaction with the emulsion target.

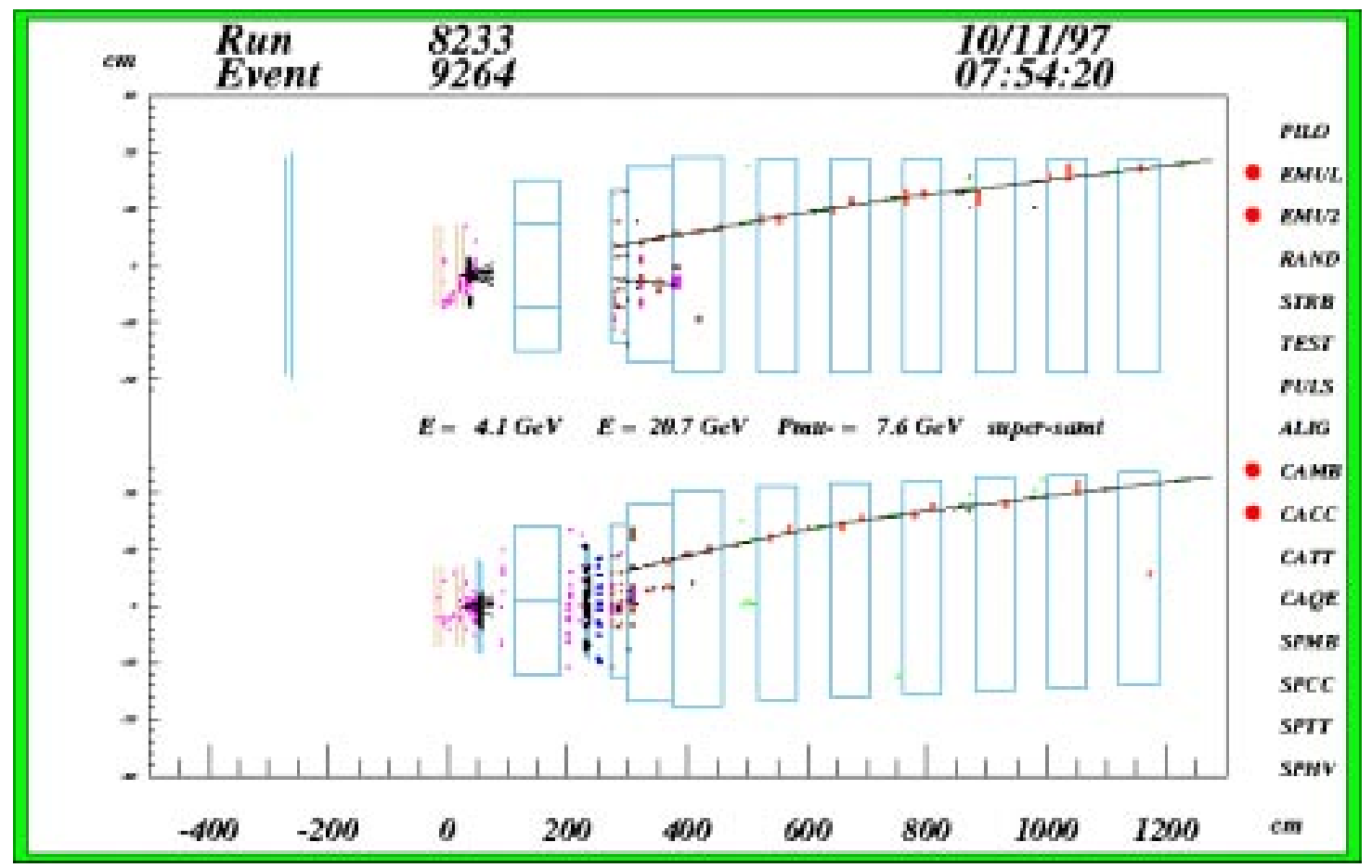

Figure 39: Event display of a neutrino interaction in the CHORUS detector. The muon from the $\nu_{\mu} \mathrm{CC}$ interaction is clearly identified.

All tracks associated with the interaction vertex having an angle smaller than 0.4 rad with respect to the beam axis are selected. In addition, given the large background of muons originating from a nearby secondary target, tracks with an angle close to the direction of this beam are rejected. Tracks are then searched for in the emulsions if their charge is negative and their momentum is in the range $1 \leq p_{h} \leq 20 \mathrm{GeV}$ and $0 \leq p_{\mu} \leq 30 \mathrm{GeV}$ for hadrons and muons, respectively. The contribution of the $\tau^{-}$leptonic decay modes not identified as such to the $0 \mu$ data sample is taken into account.

\subsubsection{Data analysis}

The scanning of the CHORUS emulsions is underway. The present status of the analysis is summarised in Table 13.

The various steps which lead to the identification of the emulsion sheet containing the vertex by means of automatic microscopes are described in $[149,150]$. They are applied to the muon for $1 \mu$ events and to all negative tracks for $0 \mu$ events. A track found in the interface emulsion is followed upstream in the emulsion target by using track segments reconstructed by automatic scanning in the most upstream $100 \mu \mathrm{m}$ of each plate. This procedure is illustrated in Fig. 40.

The vertex plate is defined as the plate where the track apparently disappears. It can be the plate 

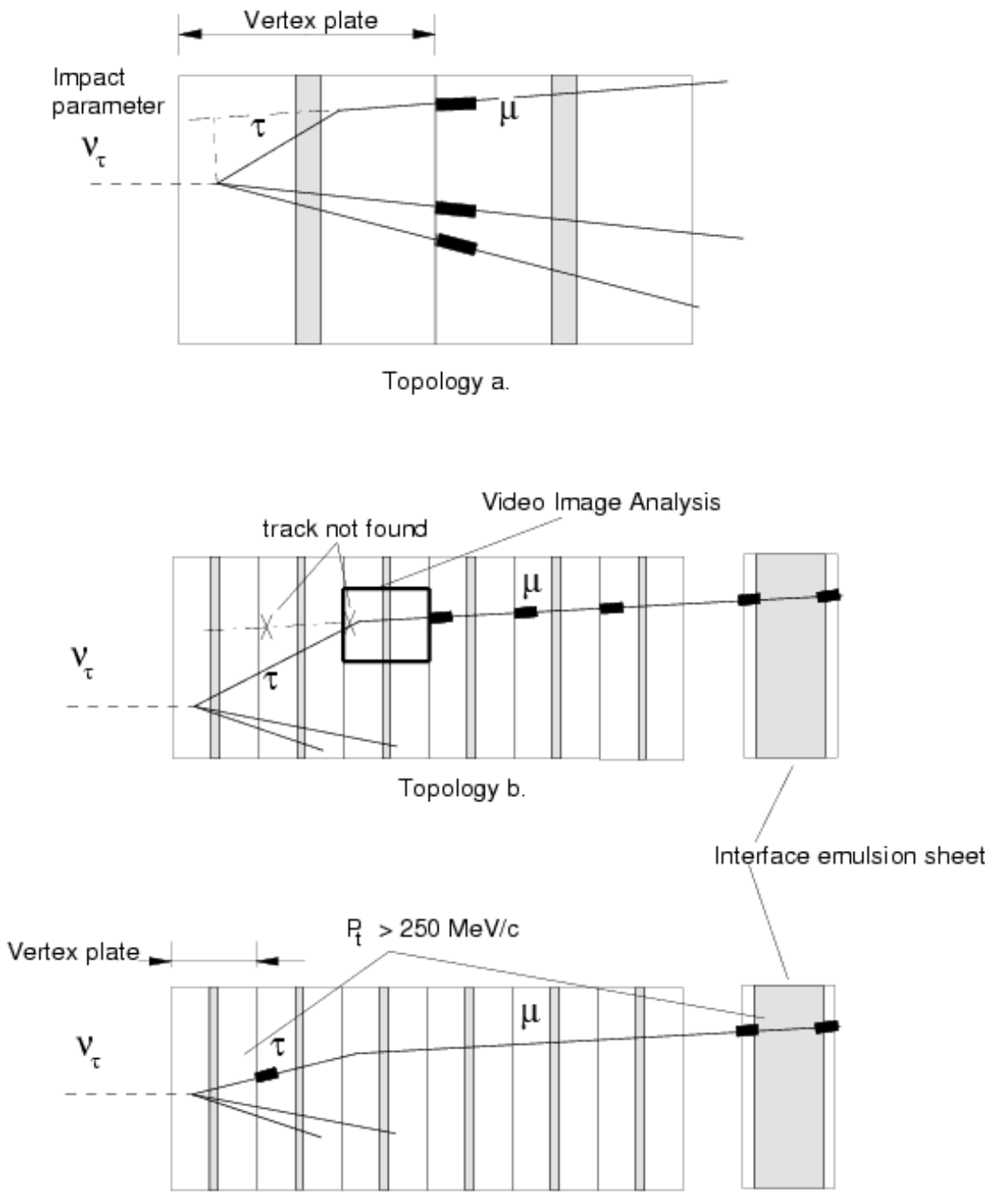

Topology c.

Figure 40: Search for a one-prong $\tau$ decay topology (kink) in the CHORUS emulsions. Different decay possibilities are shown (for details we refer to [149]). 


\begin{tabular}{||c|c|c|c|c|c||}
\hline & 1994 & 1995 & 1996 & 1997 & All \\
\hline \hline pot & $0.81 \times 10^{19}$ & $1.20 \times 10^{19}$ & $1.38 \times 10^{19}$ & $1.67 \times 10^{19}$ & $5.06 \times 10^{19}$ \\
\hline Emulsion triggers & 420000 & 550000 & 620000 & 720000 & 2310000 \\
\hline $1 \mu$ to be scanned & 67000 & 110000 & 130000 & 150000 & 4570000 \\
\hline $0 \mu$ to be scanned & 18000 & 28000 & 32000 & 38000 & 116000 \\
\hline $1 \mu$ scanned & $69 \%$ & $47 \%$ & $76 \%$ & $72 \%$ & $66 \%$ \\
\hline $0 \mu$ scanned & $60 \%$ & $48 \%$ & $73 \%^{*}$ & $51 \%^{*}$ & $55 \%^{*}$ \\
\hline $1 \mu$ located vertices & 19581 & 21809 & 38919 & 45920 & 126229 \\
\hline $0 \mu$ located vertices & 3491 & 4023 & $6758^{*}$ & $5164^{*}$ & $19436^{*}$ \\
\hline \hline
\end{tabular}

Table 13: Current status of the CHORUS analysis. For the event sample denoted by an asterix the decay search is not yet completed.

containing either the primary neutrino vertex or the secondary (decay) vertex from which the track originates (Fig. 40). The three most downstream plates of each stack are used to validate the matching with the interface sheets and are not considered as possible vertex plates. Fig. 41 shows a neutrino interaction in the CHORUS emulsion target as reconstructed by the computer.

The mean efficiency of the above scan-back procedure is $30 \%$ and $40 \%$ for $0 \mu$ and $1 \mu$ events, respectively. A simulation of the scanning procedure shows that the difference in efficiency mainly reflects the poorer quality of the hadron track predictions at the interface sheets, due to the difficulty in reconstructing the trajectory inside a dense hadronic shower. The $\mu$ decay channel allows in general a better analysis.

Once the vertex plate is found, automatic microscope measurements are performed to select the events compatible with a one-prong decay topology (kink). Different algorithms can be applied. They are described in $[149,150]$ and briefly recalled here. In the first step the event is selected either if the scan-back track has a significant impact parameter with respect to the other predicted tracks or if the change in the scan-back track direction between the vertex plate and the exit point from the emulsions corresponds to an apparent transverse momentum $p_{T}$ larger than $250 \mathrm{MeV}$. For selected events and for those with only one predicted track, digital images of the vertex plate are recorded and are analysed off-line to look for a decay kink. The second step is restricted to the search for long decay paths. In this case it is assumed that the vertex plate contains the decay vertex of a charged parent particle produced in a more upstream plate.

A computer assisted eye-scan is performed for events selected by either one of the above procedures in order to identify the presence of a secondary vertex and measure its topology. A $\tau^{-}$decay candidate must satisfy the following criteria

- the secondary vertex has to appear as a kink without blackprongs, nuclear recoils, blobs or Auger electrons: in that case the kink would be due to a re-interaction and not to a decay;

- the transverse momentum of the decay muon (hadron) with respect to the parent direction has to be larger than $250 \mathrm{MeV}$. This allows to reject $\pi$ and $K$ meson decays;

- the kink in $0 \mu$ events must occur within 3 plates downstream of the vertex plate. The kink search for the muonic channel is extended up to 5 plates in consideration of the lower background. This produces a gain in efficiency of about $8 \%$. 


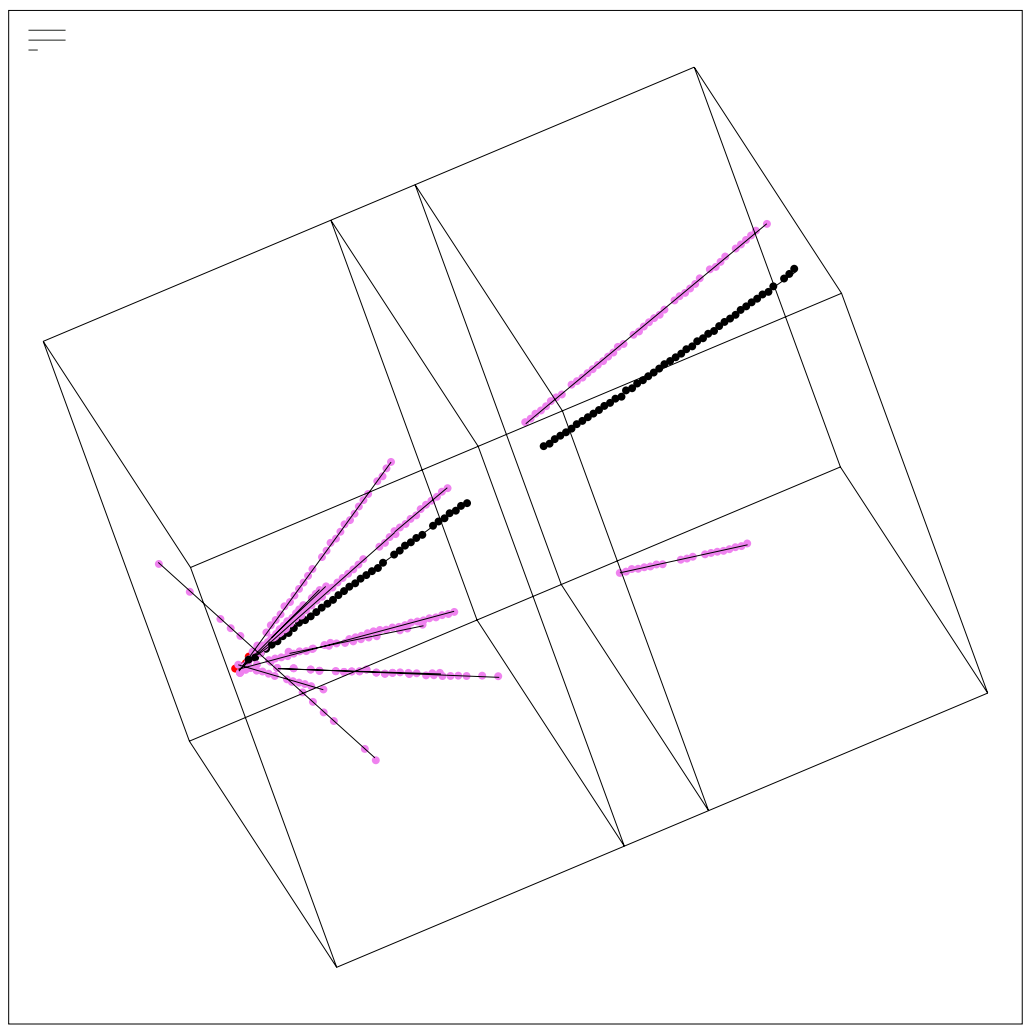

Figure 41: Neutrino interaction in the CHORUS emulsions. Two $350 \mu \mathrm{m}$ thick emulsion layers are placed on both sides of a $90 \mu \mathrm{m}$ thick plastic base. A background track uncorrelated to the event is also shown. 
No $\tau^{-}$decay candidates have been found satisfying the selection criteria. The kink finding efficiency $\epsilon_{k i n k}$ is evaluated by Monte Carlo simulations and experimentally checked by looking at hadron interactions and charm decays. About $55 \mathrm{~m}$ of hadron tracks were scanned in the emulsions. 21 neutrino interaction events with a hadron interaction were detected in the decay search procedure. This result is in good agreement with the expected value of $24 \pm 2$ coming from the simulations.

Dimuon events originating from charm decay have also been used to support the kink finding efficiency evaluations. Part of the dimuon sample collected in 1995 and 1996 was analysed searching for the reaction $\nu_{\mu} N \rightarrow \mu^{-} C^{+} X$ with the subsequent muonic decay of the charmed meson $\left(C^{+}\right)$. Assuming a charm yield $\sigma_{\text {charm }} / \sigma_{C C}$ of about $5 \%$ [151] $22.8 \pm 3.9$ dimuon events were expected and 25 found.

As an illustration of the capability of the CHORUS detector in identifying decay kinks Fig. 42 shows the reconstruction of a peculiar event recorded by the experiment [152]. One of the tracks at the interaction vertex has two kinks identified in the emulsions. This is interpreted as the production of a $D_{s}$ meson decaying into a $\tau$, in turn undergoing a muonic decay. It is the first direct observation of a neutrino induced CC interaction with two subsequent decays of short-lived particles close to the interaction vertex.

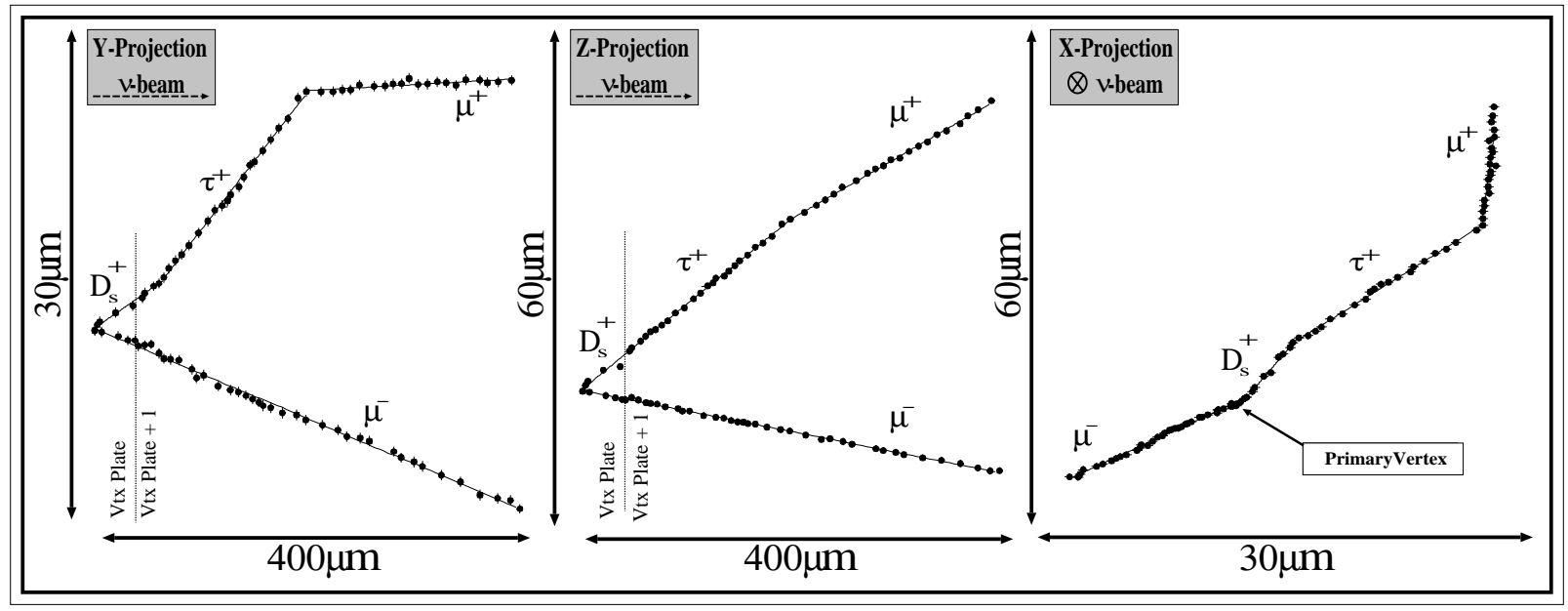

Figure 42: Double kink event reconstructed in the CHORUS emulsion target.

\subsubsection{Experiment performance}

The main sources of background to the hadronic $\tau$ decay channels are

- the production of negative charmed particles from anti-neutrinos in the beam. These events constitute a background if the primary $\mu^{+}$or $e^{+}$remain unidentified. Taking into account the appropriate crosssections and branching ratios 0.02 events are expected in the presently analysed data sample;

- the production of positive charmed mesons in CC interactions. This constitutes a background if the primary lepton is not identified and the charge of the charmed particle daughter is wrongly measured. One could expect 0.03 events from this source; 
- the associated charm production in CC and NC interactions in which one of the two charmed particles is not detected. The cross-section for charm-anticharm pair production in NC interactions relative to the total CC cross-section has been measured by the E531 experiment to be $0.13_{-0.11}^{+0.31 \%}$ [151]. The production rate of associated charm in CC interactions is unknown but an upper limit is available [151]. The estimated background from this process amounts to less than 0.01 events;

- the main background to the hadronic $\tau^{-}$decays is due to the above-mentioned hadronic white kinks mimicking a hadronic decay. Published data on white kink cross-section are scarce [153,154]. In a dedicated experiment with $4 \mathrm{GeV}$ pions at KEK [153] a steep fall-off in $p_{T}$ was measured and only one out of 58 observed kinks had a $p_{T}$ larger than $300 \mathrm{MeV}$. A Monte Carlo simulation was also performed. The results are in good agreement with the $p_{T}$ dependence of the KEK measurement. An effective white kink mean free path in emulsion $\lambda_{w k} \sim 22 \mathrm{~m}$ is obtained for a $p_{T}$ cut of $250 \mathrm{MeV}$. This result is compatible with the observation of 4 events with $p_{T}>250 \mathrm{MeV}$ at large distance from the primary vertex, along a total path of $\sim 92 m$ of scan-back tracks. This corresponds to an estimated background of 0.5 events within 3 plates downstream of the primary vertex plate. Ongoing measurements performed with hadron tracks in the CHORUS emulsions will allow to improve the estimate of the white kink background.

The principal source of background to the muonic $\tau$ decay is given by charm production. Less than 0.1 events are expected from anti-neutrinos

$$
\bar{\nu}_{\mu}\left(\bar{\nu}_{e}\right) N \rightarrow \mu^{+}\left(e^{+}\right) D^{-} X
$$

followed by $D^{-} \rightarrow \mu^{-} X^{0}$ with the $\mu^{+}\left(e^{+}\right)$escaping detection or not identified.

The prompt $\nu_{\tau}$ contamination of the beam [155] is a background common to both hadronic and muonic decay channels. The expected background is much lower than 0.1 events.

The $\tau^{-}$decay channels considered so far by CHORUS for the $\nu_{\mu} \leftrightarrow \nu_{\tau}$ oscillation search are the muonic and the one-prong hadronic. The expected number $N_{\tau i}$ of observed $\tau^{-}$decays into a channel with branching ratio $B R_{i}$ is then given by

$$
N_{\tau i}=B R_{i} \int \phi_{\mu}(E) P\left(\nu_{\mu} \leftrightarrow \nu_{\tau}\right) \sigma_{\tau} A_{\tau i} \epsilon_{\tau i} d E
$$

with $\phi_{\mu}(E)$ the incident $\nu_{\mu}$ flux spectrum, $\sigma_{\tau}$ the $\nu_{\tau}$ CC interaction cross-section, $A_{\tau i}$ the acceptance and reconstruction efficiency for the considered channel and $\epsilon_{\tau i}$ the corresponding efficiency of the decay search procedure. With proper averaging $N_{\tau i}$ can be written as

$$
N_{\tau i}=B R_{i} n_{i}\left\langle P\left(\nu_{\mu} \leftrightarrow \nu_{\tau}\right)\right\rangle \frac{\left\langle\sigma_{\tau}\right\rangle}{\left\langle\sigma_{\mu}\right\rangle} \frac{\left\langle A_{\tau i}\right\rangle}{\left\langle A_{\mu}\right\rangle}\left\langle\epsilon_{\tau i}\right\rangle
$$

where

- $n_{1}=N_{\mu}$, i.e. the number of located $\nu_{\mu} \mathrm{CC}$ interactions corresponding to the considered event sample and $n_{2}=n_{3}=n_{4}=\left(N_{\mu}\right)_{0 \mu}$, i.e. the product of $N_{\mu}$ by the relative fraction of the $0 \mu$ sample for which the analysis has been completed; 
- $\left\langle\sigma_{\mu(\tau)}\right\rangle=\int \frac{d \sigma_{\mu(\tau)}}{d E} \phi_{\mu}(E) d E$. QE interactions, resonance production and deep-inelastic interactions are taken into account with $\sigma\left(\frac{\left\langle\sigma_{\tau}\right\rangle}{\left\langle\sigma_{\mu}\right\rangle}\right)_{\text {syst }} \sim 7 \%$

- $\left\langle A_{\mu(\tau i)}\right\rangle=\int \frac{d \sigma_{\mu(\tau)}}{d E} A_{\mu(\tau i)} \phi_{\mu}(E) d E$ with a systematic error $\sigma\left(\frac{\left\langle A_{\tau i}\right\rangle}{\left\langle A_{\mu}\right\rangle}\right)_{s y s t} \sim 7 \%$;

- $\left\langle\epsilon_{\tau i}\right\rangle$ is the average efficiency of the decay search procedure for the accepted events $\left(\sigma\left(\left\langle\epsilon_{\tau i}\right\rangle\right)_{s y s t} \sim\right.$ $10 \%)$

In combining the results from the $1 \mu$ and $0 \mu$ event samples one can define $N_{\mu}^{e q}$, i.e. the equivalent number of muonic events for the $0 \mu$ sample [150]. In the absence of a positive signal the $90 \%$ C.L. upper limit on the oscillation probability becomes

$$
P\left(\nu_{\mu} \leftrightarrow \nu_{\tau}\right) \leq \frac{2.38 r_{\sigma} r_{A}}{B R_{\mu}\left\langle\epsilon_{\tau \mu}\right\rangle\left[N_{\mu}+N_{\mu}^{e q}\right]}
$$

where $r_{\sigma}=\left\langle\sigma_{\mu}\right\rangle /\left\langle\sigma_{\tau}\right\rangle$ and $r_{A}=\left\langle A_{\mu}\right\rangle /\left\langle A_{\tau \mu}\right\rangle$. The numerical factor 2.38 takes into account the total systematic error of $17 \%$ according to the prescription given in [156]. Within the present statistics the $90 \%$ C.L. upper limit obtained by CHORUS is [37]

$$
P\left(\nu_{\mu} \leftrightarrow \nu_{\tau}\right) \leq 4.0 \times 10^{-4}
$$

The corresponding excluded region in the oscillation parameter space is shown for Fig. 32 for the twoflavour mixing scheme. Maximum mixing between $\nu_{\mu}$ and $\nu_{\tau}$ is excluded at the $90 \%$ C.L. for $\Delta m_{\mu \tau}^{2}>$ $0.8 \mathrm{eV}^{2}$. Large $\Delta \mathrm{m}^{2}$ values are excluded for $\sin ^{2} 2 \theta_{\mu \tau}>8.0 \times 10^{-4}$. The experiment is expected to eventually reach the limit of $\sin ^{2} 2 \theta_{\mu \tau} \sim 1-2 \times 10^{-4}$ for large $\Delta m^{2}$.

\subsection{The NOMAD experiment}

The NOMAD experiment $[36,157]$ has been searching for $\nu_{\tau} \leftrightarrow \nu_{\mu}$ oscillations in the CERN WANF beam. The approach adopted by the experiment for the $\tau$ detection is complementary to the one used by CHORUS. It is based on the measurement of the missing transverse momentum in the final state; one exploits the different kinematics of $\nu_{\tau}$ interactions with respect to $\nu_{\mu, e}$ interactions.

The above method imposes two conflicting requirements to the detector design. The first is that the target material has to have low density and low $Z$ to allow for precise measurement of charge and momentum of the particles in the event. On the other hand, the target has to be massive to guarantee the collection of a significant number of neutrino interactions.

The NOMAD detector [158] is shown in Fig. 43. It is housed inside the former UA1 magnet which provides a dipolar magnetic field of $0.4 T$ perpendicular to the beam axis over a volume of $3.5 \times 3.5 \times 7.5 \mathrm{~m}^{3}$. The requirement of a massive and low density target is fulfilled by the use of a 2.7 ton active target made of drift chambers with an average density of $0.1 \mathrm{~g} / \mathrm{cm}^{3}$. The chambers provide a momentum resolution of $\sigma_{p} / p \approx 0.05 / \sqrt{L} \oplus 0.008 p / \sqrt{L^{5}}$ where $L$ is the track length in meters and $p$ is the momentum expressed in $\mathrm{GeV}$. 


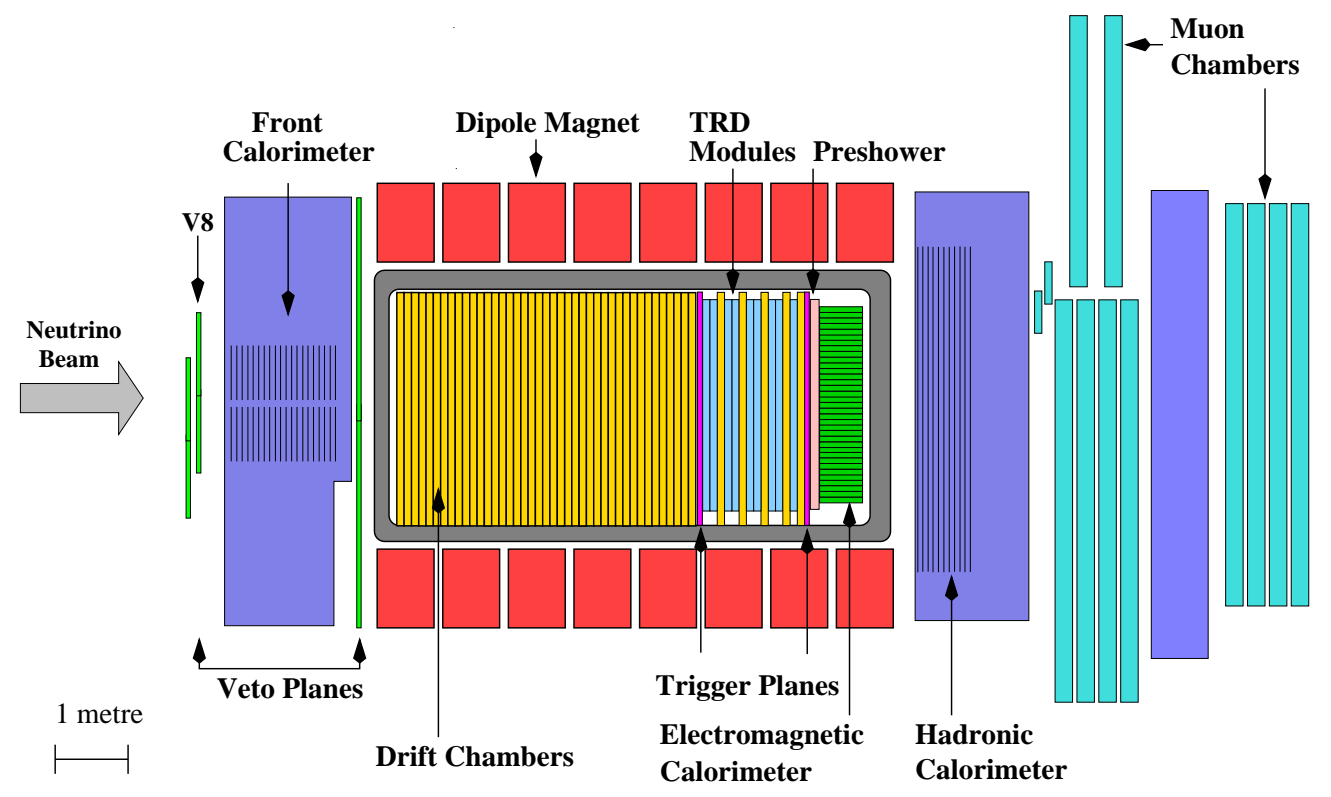

Figure 43: Side view of the NOMAD detector.

The drift chambers are followed by 9 transition radiation detectors (TRD) each made of a polypropylene radiator followed by a plane of straw tubes. The TRD allows to reach a pion rejection factor of 1000 for isolated tracks in the momentum range $1-50 \mathrm{GeV}$ with $90 \%$ electron detection efficiency.

A preshower consisting of a $1.6 X_{0}$ thick lead converter followed by a horizontal and a vertical plane of proportional streamer tubes is placed downstream of the TRD. It precedes an electromagnetic calorimeter made of an array of lead glasses (19 $X_{0}$ thick). The energy resolution of the preshower/electromagnetic calorimeter is $\sigma(E) / E=3.2 \% \sqrt{E(G e V)} \oplus 1 \%$. The combined use of TRD, preshower and calorimeter allows to improve the electron identification against pion contamination.

A hadronic calorimeter with energy resolution of $\sigma(E) / E=100 \% / \sqrt{E(G e V)}$ is located outside the magnet. It is followed by two large drift chambers. The first one is placed after $8 \lambda_{\text {int }}$ and the second after $13 \lambda_{\text {int }}$ of iron. This system provides the required muon identification.

Fig. 44 shows the display of a WANF beam neutrino-interaction reconstructed in the NOMAD detector. The event is a candidate for a $\nu_{e} \mathrm{CC}$ interaction in the target.

\subsubsection{Data analysis}

The NOMAD experiment started data taking in 1995. In the $1995-1998$ running period $1.2 \times 10^{6}$ events were acquired with a muon identified in the final state, corresponding to $5 \times 10^{19}$ pot.

The track reconstruction of charged particles is performed by using hits in the drift chambers. The momentum is estimated by means of a Kalman filter algorithm which takes into account the map of the magnetic field, the energy loss and the multiple scattering in the traversed material [158]. The neutrino 


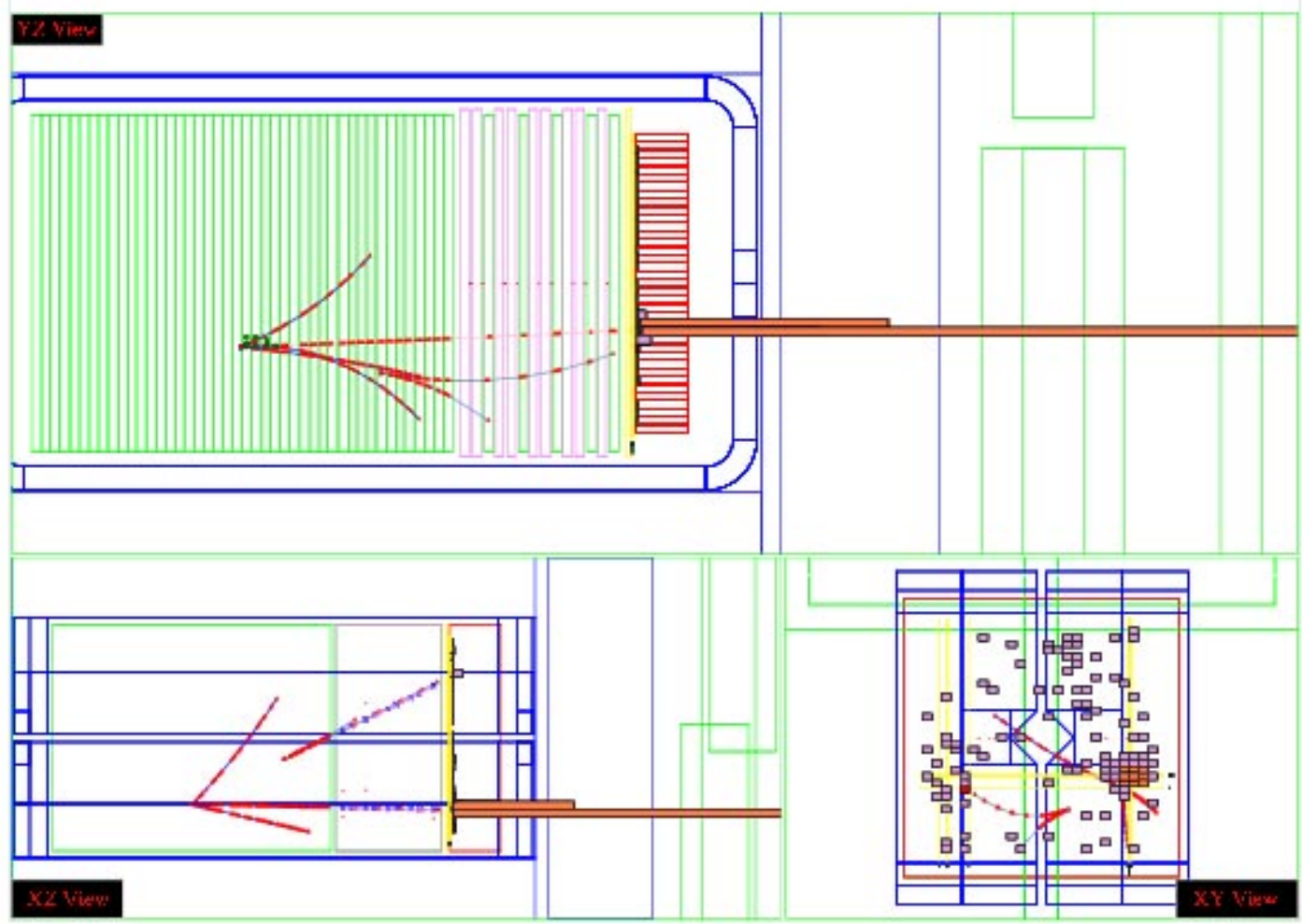

Figure 44: Event display of a candidate $\nu_{e}$ interaction in the NOMAD detector. The electron from the $\nu_{e}$ $\mathrm{CC}$ interaction is identified as the track producing a large signal in the downstream lead glass calorimeter. The longitudinal and the transverse projections w.r.t. the beam are shown. 
interaction vertex is reconstructed if at least one track with momentum larger than $500 \mathrm{MeV}$ is detected. The kinematical analysis is based on the reconstruction of the following variables: $Q^{2}, W^{2}$, the Bjorken $x$ and $y$, the total visible energy $E_{v i s}$ and the transverse missing momentum $p_{T}$.

In the $\nu_{\mu} \mathrm{CC}$ data sample, in which in principle all final-state charged particles are detected, the $p_{T}$ value is expected to be small. It is basically due to the Fermi motion of the nucleons. The average $p_{T}$ predicted by Monte Carlo is $620 \mathrm{MeV}$ but the experimental value is $820 \mathrm{MeV}$. A method, the so-called Data Simulator (DS), has been developed to overcome this discrepancy. The idea is to use the data themselves to compute the detection efficiencies for signal and background. Starting from the measured $\nu_{\mu}$ CC events, the identified muon in the final state is removed and replaced by another lepton $l\left(l=\nu_{\mu}, e, \tau\right)$ in order to obtain fake $\nu_{\mu}$ $\mathrm{NC}, \nu_{e} \mathrm{CC}$ or $\nu_{\tau} \mathrm{CC}$ event samples. These are referred to as Data Simulator sets. The same procedure is applied to the $\nu_{\mu}$ CC Monte Carlo sample (Monte Carlo Simulator: MCS). The comparison between DS and MCS samples gives a direct measurement of the systematic difference between data and simulated events, hence allowing for the evaluation of signal and background efficiencies. The $\tau^{-} \rightarrow \mu^{-} \bar{\nu}_{\mu} \nu_{\tau}$ channel is not used for the oscillation search since data simulator events cannot be built for the $\nu_{\mu}$ CC sample.

\subsubsection{Experiment performance}

\section{Background}

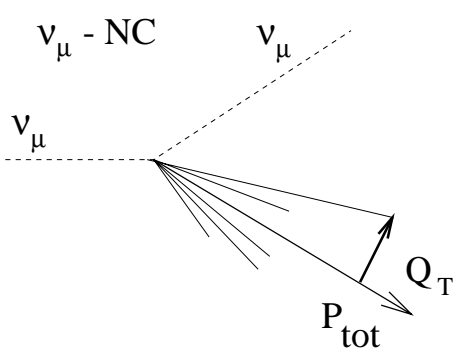

Signal

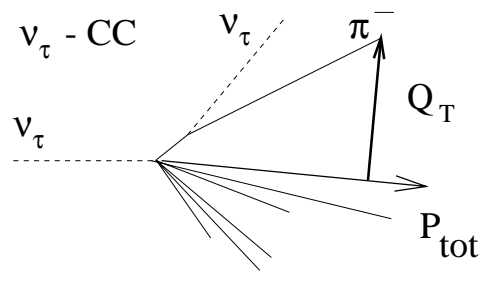

Figure 45: NOMAD: the $Q_{T}$ variable is illustrated for background and signal events.
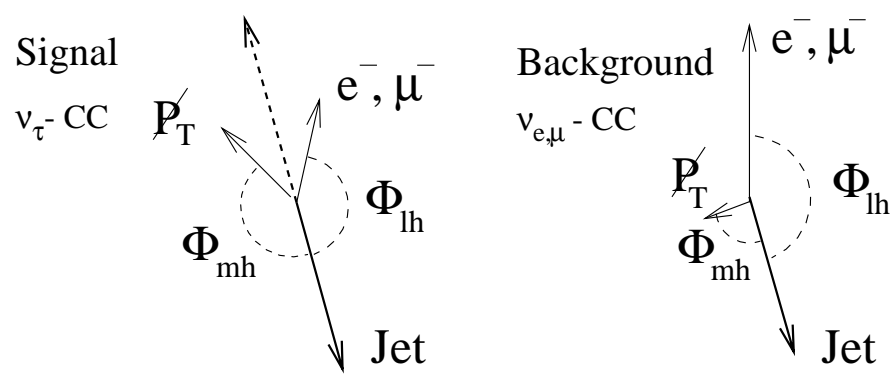

Figure 46: NOMAD: the missing transverse momentum variable is illustrated for background and signal events.

The detection of a $\nu_{\tau} \mathrm{CC}$ interaction in NOMAD proceeds by exploiting two distinctive features of the $\tau$ decay. The first is that the hadronic decay product of the $\tau$ is more isolated with respect to the hadronic jets produced at the primary vertex than hadrons in $\nu_{\mu} \mathrm{NC}$ events. A variable used to quantify 
the isolation is the component of the momentum of the visible $\tau$ decay products perpendicular to the total visible momentum vector $Q_{T}$ (Fig. 45). The second feature is that, due to the neutrino produced in the $\tau$ decay, the $p_{T}$ in a $\nu_{\tau}$ event is larger than that expected for $\nu_{\mu} \mathrm{CC}$ interactions but lower than in $\nu_{\mu} \mathrm{NC}$ events and correlated in direction with the charged decay products (Fig. 46).

Variables used to discriminate signal from background are

- the total transverse momentum $p_{T}$;

- $\phi_{m h}$ and $\phi_{l h}$, the angles between the missing momentum and the hadronic jet and between the lepton and the hadronic jet, respectively;

- the transverse mass $M_{T}$ defined as $M_{T}=\sqrt{\left(\left|\vec{p}_{T}\right|+\left|\vec{p}_{T}^{l}\right|\right)^{2}-\left(\vec{p}_{T}^{\text {jet }}\right)^{2}}$

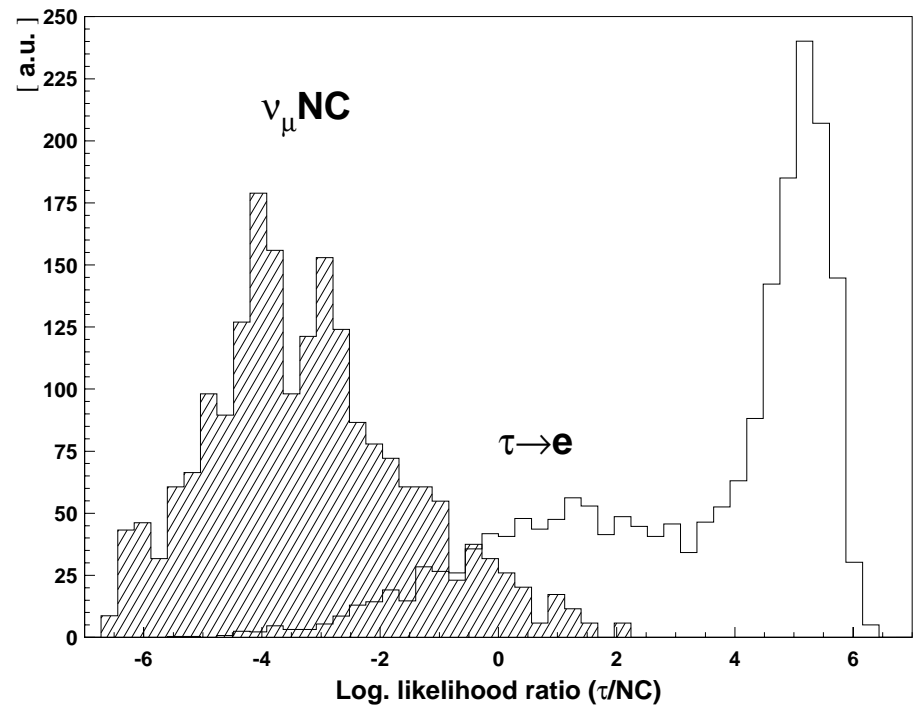

Figure 47: The likelihood ratio $L_{R}$ for the $\nu_{\mu} \mathrm{NC}$ background and the the $\tau \rightarrow e$ sample in the NOMAD analysis.

The above variables together with others $\left(X_{i}\right)$, which are discussed in [159], allow to extract a signal by building probability density functions $P\left(X_{i}\right)$ for signal and background and the likelihood $L=\prod_{i=1}^{N} P\left(X_{i}\right)$. The discrimination is obtained by evaluating the ratio $L_{R}=\log \left(L_{\text {signal }} / L_{\text {background }}\right)$. A typical example of $L_{R}$ distribution is shown in Fig. 47 .

Two distinct analyses are carried out. One uses events with at least one track at the primary vertex in addition to the $\tau$ products (the DIS analysis). The other deals with events with 0,1 or 2 additional tracks (the low multiplicity analysis, LM). The methods have been optimised by comparing Monte Carlo and Data Simulator event samples.

The number of $\tau$ events expected for unity oscillation probability is given by $N_{\tau}=N_{\mu}\left(\sigma_{\tau} / \sigma_{\mu}\right) B R \varepsilon$ (Table 14). The systematic uncertainty on $N_{\tau}$ is about 10\%. Its effect on the evaluation of the oscillation 


\begin{tabular}{||c|c|c|c||}
\hline Channel & Exp. background & $N_{o b s}$ & $N_{\tau}^{\text {max }}$ \\
\hline \hline$\tau^{-} \rightarrow e^{-} \nu_{\tau} \bar{\nu}_{e}$ DIS & $5.3_{-0.6}^{+0.8}$ & 5 & 4110 \\
$\tau^{-} \rightarrow e^{-} \nu_{\tau} \bar{\nu}_{e}$ LM & $5.4 \pm 0.9$ & 6 & 859 \\
\hline$\tau^{-} \rightarrow h^{-}\left(n \pi^{0}\right) \nu_{\tau}$ DIS & $6.8 \pm 2.1$ & 6 & 2232 \\
$\tau^{-} \rightarrow h^{-}\left(n \pi^{0}\right) \nu_{\tau}$ LM & $6.7 \pm 2.3$ & 5 & 357 \\
$\tau^{-} \rightarrow \rho^{-} \nu_{\tau}$ DIS & $7.3_{-1.2}^{+2.2}$ & 9 & 2547 \\
$\tau^{-} \rightarrow \rho^{-} \nu_{\tau}$ LM & $5.2 \pm 1.8$ & 7 & 458 \\
\hline$\tau^{-} \rightarrow \pi^{-} \pi^{-} \pi^{+} \nu_{\tau}$ DIS & $6.5 \pm 1.1$ & 5 & 1180 \\
$\tau^{-} \rightarrow \pi^{-} \pi^{-} \pi^{+} \nu_{\tau}$ LM & $0.4_{-0.4}^{+0.6}$ & 0 & 108 \\
\hline \hline Total & $43.6 \pm 4.5$ & 43 & 11851 \\
\hline
\end{tabular}

Table 14: Current status of the NOMAD analysis. $N_{\tau}^{\max }$ is defined as $N_{\tau}^{\max }=N_{\mu}^{o b s} \times\left(\sigma_{\tau} / \sigma_{\mu}\right) \times B R \times$ $\left(\varepsilon_{\tau} / \varepsilon_{\mu}\right)$.

limits in the Feldman and Cousins approach [76] is negligible. No positive oscillation signal has been found so far by NOMAD. The resulting $90 \%$ C.L. upper limit on the oscillation probability is [38]

$$
P\left(\nu_{\mu} \leftrightarrow \nu_{\tau}\right) \leq 4.2 \times 10^{-4}
$$

The excluded region at the 90\% C.L. is graphically shown in Fig. 32 .

Thanks to its good electron identification NOMAD is also sensitive to $\nu_{\mu} \leftrightarrow \nu_{e}$ oscillations [160]. Since the oscillation signal would manifest itself as a distortion of the $\nu_{e}$ spectrum, an important requirement is the precise knowledge of the beam composition. In order to reduce systematic errors due to beam simulations, an empirical parametrisation of the neutrino spectra by using real data is performed.

The method applied exploits the fact that, due to the small $\bar{\nu}$ contaminations, no appreciable signal is expected in the $\bar{\nu}_{\mu} \leftrightarrow \bar{\nu}_{e}$ channel. Therefore, one can in principle extract the $\nu_{e}$ spectrum from the $\nu_{\mu}, \bar{\nu}_{\mu}$ and $\bar{\nu}_{e}$ spectra. In fact, $\nu_{e}$ come mainly from $K^{+}$and $K_{L}^{0}$ decays (Fig. 48). The $K^{+}$spectrum can be constrained by the high energy part of the $\nu_{\mu}$ spectrum (Fig. 48). In a similar way, the $K_{L}^{0}$ spectrum can be derived from the measured energy distributions for $\bar{\nu}_{\mu}$ and $\bar{\nu}_{e}$. Once the contributions from the parent particles are known the expected $\nu_{e}$ energy distribution in the absence of oscillations can be derived.

The ratio $\mathcal{R}_{e \mu}$ between the number of $\nu_{e} \mathrm{CC}$ and $\nu_{\mu} \mathrm{CC}$ interactions as a function of the total energy is used in order to reduce the systematic errors. The search for $\nu_{\mu} \leftrightarrow \nu_{e}$ oscillations is performed by comparing the $\mathcal{R}_{e \mu}$ distributions for data and simulated events. Preliminary results show no evidence for oscillations. The high $\Delta m^{2}$ values allowed by the LSND result are excluded down to $\sim 8 \mathrm{eV}^{2}$.

\section{$8 \quad \nu_{\mu} \leftrightarrow \nu_{e}$ oscillation searches at BNL}

Neutrino oscillation experiments have been performed at the Brookhaven National Laboratory (BNL) by using the Alternating Gradient Syncrotron (AGS) neutrino beam [162]. $28.5 \mathrm{GeV}$ protons $\left(1.5 \times 10^{13}\right.$ pot per $1.2 \mathrm{~s}$ ) from the AGS are sent onto an alloy target made of $75 \%$ tungsten and $25 \%$ rhenium. The energy distribution of the secondary pions is peaked around $3 \mathrm{GeV}$ giving an almost pure $\nu_{\mu}$ beam with an energy spectrum centered at $\sim 1 G e V$. The integrated flux ratio $\nu_{e} / \nu_{\mu}\left(\bar{\nu}_{e} / \bar{\nu}_{\mu}\right)$ is estimated to be 

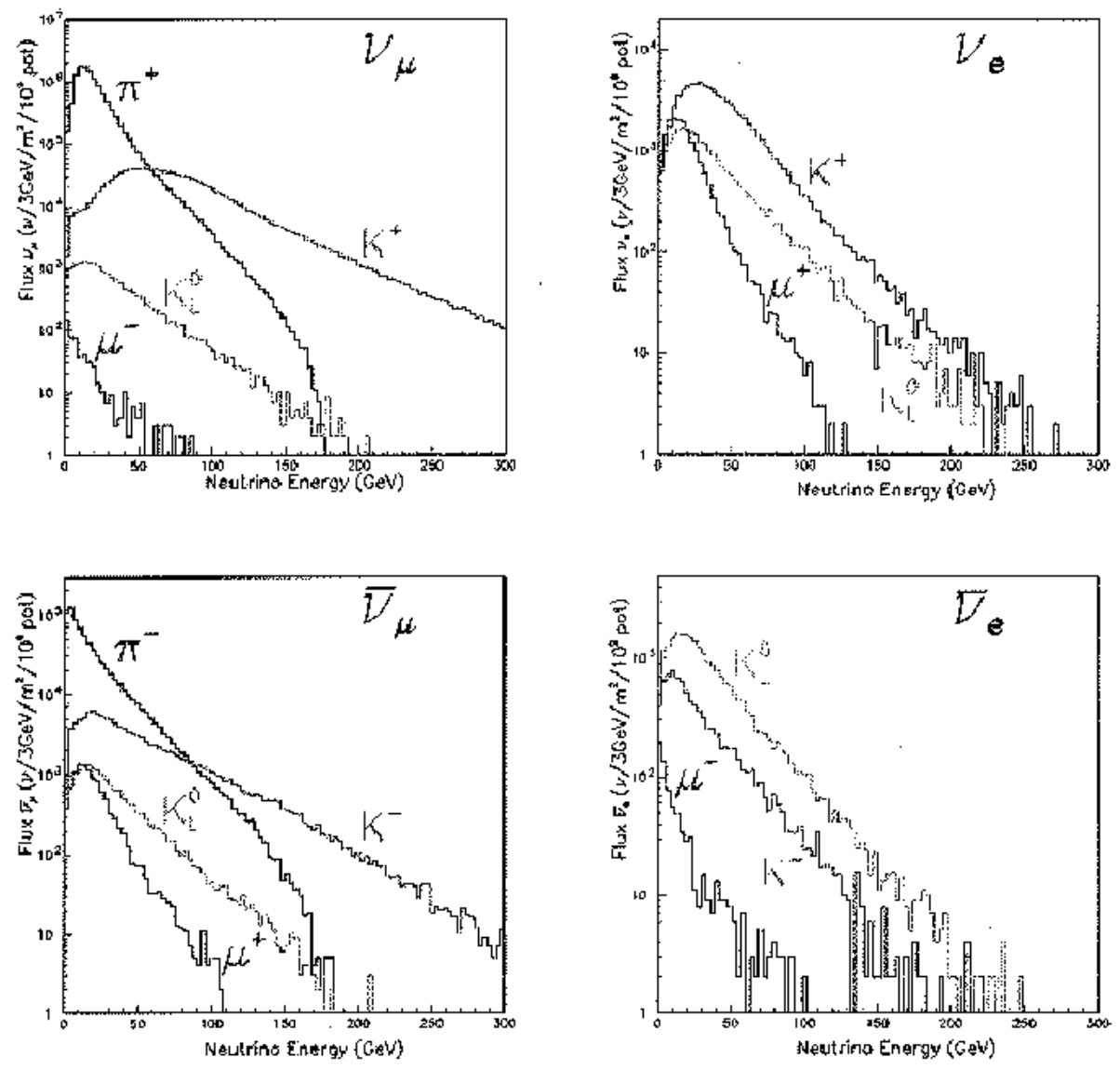

Figure 48: Energy distributions of the hadrons producing the different components of the WANF neutrino beam. 
$6.8 \times 10^{-3}\left(6.3 \times 10^{-3}\right)$ with an error of $10 \%$ due to uncertainties in the kaon production models.

The Brookhaven experiments E734 and E776 experiments are described in some detail in the following Sections. Here we also mention the E816 experiment which exploited the AGS beam for a $\nu_{\mu} \leftrightarrow \nu_{e}$ oscillation search [163]. The E816 detector consists of a fine-grained calorimeter made of flash-tube planes interspaced with $3 \mathrm{~cm}$ thick iron plates. Scintillator hodoscopes provide the trigger for the flash-tubes. The overall dimensions of the calorimeter are about $6 \times 3 \times 3 \mathrm{~m}^{3}$. Electron events possibly induced by electron neutrinos are identified by their showering inside the calorimeter. The main background is given by photon induced showers from $\pi^{0}$ s. No statistically significant signal was observed by the experiment: an excess of $10 \pm 12$ signal electron events over the estimated background was found.

E816 was motivated by the results of the former PS191 experiment [164] exposed to the CERN PS neutrino beam and exploiting an analogous experimental method. This experiment observed an eccess of electron events corresponding to a signal of about three standard deviations. The result of both experiments were assumed to be affected by systematic errors arising from a quantitatively unknown behaviour of the low energy cross-section not taken into account in the simulations [163].

\subsection{The BNL-E734 experiment}

The E734 neutrino detector $[162,165,166]$ consists of 112 planes of liquid scintillator cells (1792 per plane) interleaved with 224 planes of proportional drift tubes. Each plane has $4 \times 4 \mathrm{~m}^{2}$ transverse dimensions and is $8 \mathrm{~cm}$ thick. The dimensions of the cells are $26 \times 425 \times 8 \mathrm{~cm}^{3}$. The fine segmentation of the detector and the scintillator detection technique provide a good reconstruction of the event topology, adequate identification of electromagnetic showers and effective electron/photon and pion/proton discrimination through the measurement of $d E / d x$.

A shower counter $12 X_{0}$ thick $\left(4 \times 4 \mathrm{~m}^{2}\right)$ is placed immediately downstream of the target detector providing additional containment for electromagnetic showers originating at the end of the target. It is followed by a magnet of $1.8 \times 1.8 \times 0.46 \mathrm{~m}^{3}$ aperture which allows the study of low $q^{2}$ QE reactions.

The search for $\nu_{\mu} \leftrightarrow \nu_{e}$ oscillations is performed by counting the number of $\nu_{e} n \rightarrow e^{-} p$ and $\nu_{\mu} n \rightarrow \mu^{-} p$ events. The first sample gives a measurement of the $\nu_{e}$ flux $\phi_{e}(E)$ while the second is related to the $\nu_{\mu}$ flux $\phi_{\mu}(E)$. The $e^{-} p$ sample is extracted from the entire exposure of $0.9 \times 10^{19}$ pot.

$e^{-} p$ events are pre-selected by requiring an electromagnetic shower with an angle smaller than $240 \mathrm{mrad}$ with respect to the neutrino beam direction. The final selection is accomplished by an eye-scan in order to reject events with more than one electromagnetic shower or with an interacting hadron. Events with an upstream vertex are assumed to be photon-induced showers and used as a control sample. $873 e^{-} p$ events are left after requiring the electron energy $E_{e}$ to be in the range $0.21-5.1 \mathrm{GeV}$. The energy distribution is shown in Fig. 49a.

The following processes are backgrounds to the reaction $\nu_{e} n \rightarrow e^{-} p$
a) $\nu_{\mu} p \rightarrow \nu_{\mu} p \pi^{0} ; \nu_{\mu} n \rightarrow \nu_{\mu} n \pi^{0}$
b) $\nu_{e} p \rightarrow e^{-} p \pi^{+} ; \nu_{e} n \rightarrow e^{-} n \pi^{+}$
c) $\nu_{\mu} e^{-} \rightarrow \nu_{\mu} e^{-}$

Events from a) are characterised either by photon showers associated with a significant energy deposition or by a vertex upstream of the shower. Their energy distribution is shown in Fig. 49b. On the other hand, due to the $240 \mathrm{mrad}$ cut, one expects the $e^{-} p$ events below $0.9 \mathrm{GeV}$ to be mostly due to photons, to a 


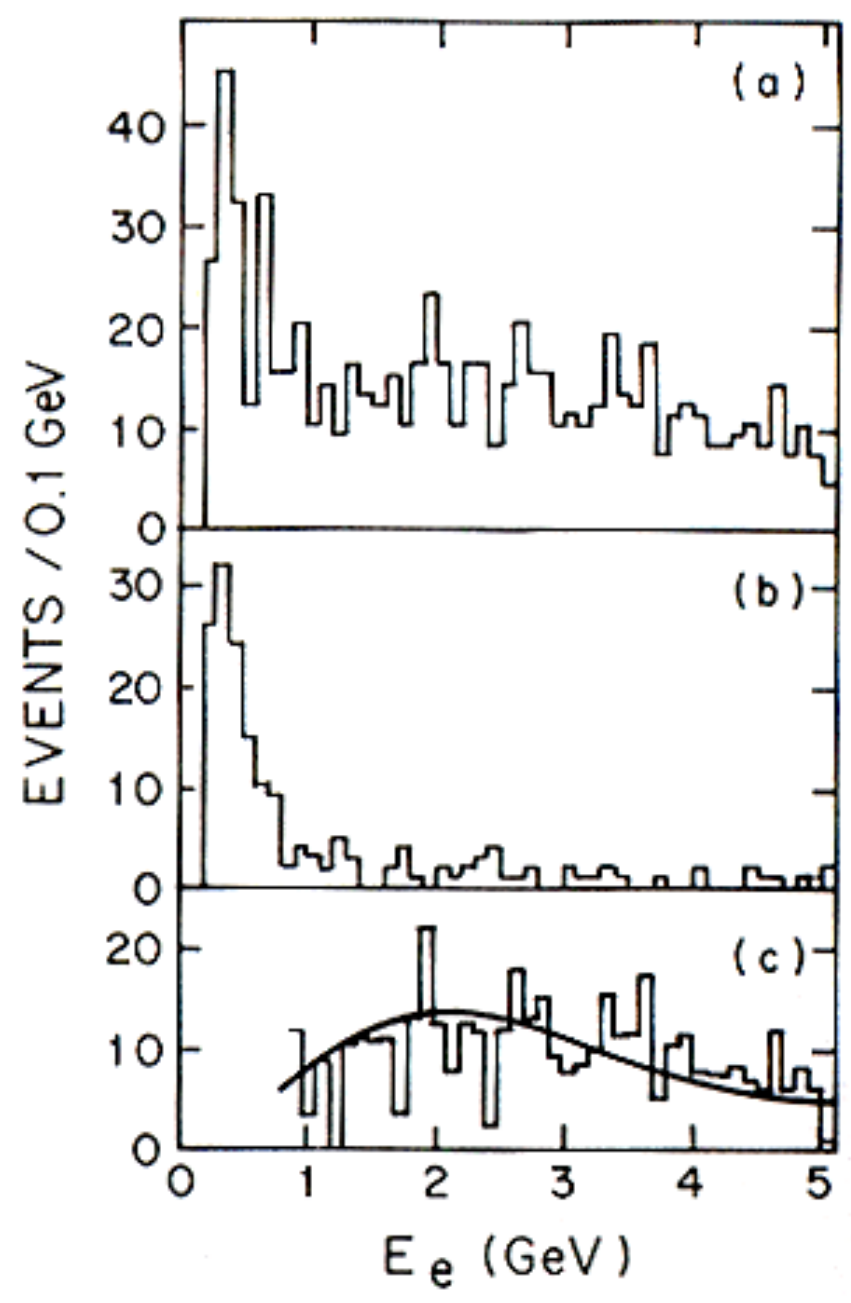

Figure 49: (a) Shower energy distribution of all $\nu_{e} n \rightarrow e^{-} p$ candidate events in the E734 experiment. (b) Energy distribution of photons recognised as coming from an upstream event vertex. (c) The resulting $e^{-} p$ energy distribution after background subtraction. The solid curve is the energy distribution of $e^{-}$from $\nu_{e} n \rightarrow e^{-} p$ as evaluated by Monte Carlo. 
lesser extent to electrons from $\nu_{\mu} e \rightarrow \nu_{\mu} e$ and only marginally to electrons from $\nu_{e} n \rightarrow e^{-} p$. This makes it possible to subtract a) events from the $e^{-} p$ sample with more than $0.9 \mathrm{GeV}$.

Reactions b) can be recognised and subtracted by looking at the delayed signal from the $\pi-\mu-e$ chain. 653 events from the initial sample have $E_{e}>0.9 \mathrm{GeV}, 20 \%$ of them due to processes b) and $13 \%$ to c). A further subtraction is needed to remove events induced by c) and $\nu_{e} n \rightarrow e^{-} p \pi^{0}$. The final $e^{-} p$ sample contains 418 events with $0.9<E_{e}<5.1 \mathrm{GeV}$ and their energy distribution is shown in Fig. 49c.

$\mu^{-} p$ events are extracted from an exposure of $0.14 \times 10^{19}$ pot. They are selected by requiring two prongs. One of them has to be identified (by range and ionisation) as a proton while the other is allowed to escape from the detector. After background subtraction, mostly due to single $\pi^{+}$CC production, the $\mu^{-} p$ sample is left with 1370 events.

The flux of neutrinos $\left(\nu_{e}\right.$ and $\left.\nu_{\mu}\right)$ as a function of the energy is given by

$$
\phi_{l}(E)=\frac{\mathrm{Nb} . \text { of observed QE events between } E \text { and } E+\Delta E}{\sigma_{Q E} a^{l p}(E) \Delta E N_{\text {pot }}(\text { Nb. of target atoms })}\left(\frac{\nu_{l}}{G e V m^{-2}\left(10^{13} N_{\text {pot }}\right)}\right)
$$

where $l=e$ or $\mu, a^{l p}(E)$ are acceptance functions, $\sigma_{Q E}$ the QE cross-section, $N_{p o t}$ the number of pot. The acceptance functions $a^{l p}(E)$ are estimated by simulations. The corresponding $Q^{2}$ distribution is found in good agreement with expectations after applying the acceptance function to the data.

Neutrino oscillations are searched for by computing the quantity

$$
\left.\frac{\phi_{e}(E)}{\phi_{\mu}(E)}\right|_{o b s}-\left.\frac{\phi_{e}(E)}{\phi_{\mu}(E)}\right|_{e x p}
$$

The above difference is expected to be zero in the absence of oscillations. The search yields a negative result. By using data between 0.9 and $5.1 \mathrm{GeV}$ the following limits are obtained: $\sin ^{2} 2 \theta \leq 3.4 \times$ $10^{-3}$ (for large $\Delta m^{2}$ values) and $\Delta m^{2}<0.43 e V^{2}$ (for $\sin ^{2} 2 \theta=1$ ).

\subsection{The BNL-E776 experiment}

The E776 experiment $[167,168]$ searched for $\nu_{\mu} \leftrightarrow \nu_{e}\left(\bar{\nu}_{\mu} \leftrightarrow\left(\bar{\nu}_{e}\right)\right)$ oscillations by looking for an excess of $\nu_{e}\left(\bar{\nu}_{e}\right)$ within an almost pure $\nu_{\mu}\left(\bar{\nu}_{\mu}\right)$ beam. The beam line horn magnetic field was set to both positive and negative polarities to produce beams predominantly made of either neutrinos or anti-neutrinos.

The E776 detector consists of a fine-grained electromagnetic calorimeter followed by a muon spectrometer. The calorimeter is composed of 90 planes of proportional drift tubes interleaved with $2.54 \mathrm{~cm}$ thick concrete absorber planes (1/4 of radiation length) for a total mass of 230 ton. Consecutive tube planes are placed orthogonally. Every tenth plane of concrete is replaced by a plane of scintillators used for timing. Each plane of drift tubes includes $64(5.49 \mathrm{~m} \times 8.26 \mathrm{~cm} \times 3.91 \mathrm{~cm})$ aluminium tubes.

The electromagnetic energy resolution of the calorimeter is $20 \% \sqrt{E}$ and its angular resolution $2^{\circ}$. The muon spectrometer is made of tracking chambers and of a toroidal magnet with a $18 k G$ field. The mean energy of the beam is $1.4 \mathrm{GeV}$ and the distance of the detector from the proton target is $1 \mathrm{~km}$.

The event sample analysed for the oscillation search corresponds to $\sim 1.5 \times 10^{19}$ pot with each of the polarities. The $\nu_{\mu}$ analysis is aimed at the measurement of the $\nu_{\mu}$ flux needed to normalise the $\nu_{e}$ data; it makes use of QE events. The final muon sample is selected by applying the following cuts 
- the interaction vertex is required to be inside the fiducial volume;

- the primary track has to be either contained in the calorimeter or pass through the muon spectrometer;

- the primary track has to be longer than three interaction lengths (for hadron rejection);

- the angle of the primary track is required to be such that $\cos \theta \leq 0.8$;

- the number of hits not associated with the primary track has to be $\leq 5$ (to reject multi-prong events).

The resulting muon sample consists of 6676 (3065) events for positive (negative) horn polarity. Cosmicray contamination is negligible. The background due to multi-prong events is estimated to be $11 \%$ ( $8 \%$ ) in the positive (negative) polarity data.

The $\nu_{e}$ events are selected by using the same criteria as for the $\nu_{\mu}$ and by requiring the primary cluster to be contained in the calorimeter with a length larger than 15 planes. After applying these cuts 2303 (733) events survive for the positive (negative) polarity.

Additional cuts are used to reduce the contamination of electromagnetic showers induced by secondary hadron interactions and $\pi^{0}$ induced showers: 136 (47) events are then left in the positive (negative) polarity sample. The $\pi^{0}$ background is estimated to be of 94 (41) events for the positive (negative) polarity by using identified $\pi^{0}$ in the data. The number of $\nu_{e}\left(\bar{\nu}_{e}\right)$ induced events is evaluated by Monte Carlo to be 32 (3.5) for the positive and 7.7 (12.2) for the negative polarity data. The electron energy distributions of background events are shown in Fig. 50.

The final positive polarity sample contains 136 events with an expected background of $131 \pm 12$ (stat) \pm 20 (back stat) \pm 19 (syst). The negative polarity data set includes 47 events with a background of $62 \pm 8$ (stat) \pm 13 (back stat) \pm 9 (syst). Both samples are consistent with the absence of oscillations. The parameter region excluded by the experiment in the $\Delta m^{2}-\sin ^{2} 2 \theta$ plane is shown in Fig. 58 .

\section{Neutrino oscillation searches at Fermilab}

Neutrino oscillation experiments were performed at Fermilab by using bubble chamber detectors. Some of them made use of a WBB configuration [169-172]. The negative results of their searches are summarised in Table 15, where the $90 \%$ C.L. limits on $\Delta m^{2}$ and $\sin ^{2} 2 \theta$ are listed.

Other experiments exploited the Fermilab NBB [108]. One advantage related to the narrow energy band is that the expected $\nu_{e}$ flux determination only depends on the well known $K_{e 3} / K_{\mu 2}$ decay ratio, since interactions of neutrinos from $K \rightarrow \mu \nu$ decays can be separated from those of $\pi \rightarrow \mu \nu$ neutrinos. Second, the energy distribution of interactions from three-body $K_{e 3}$ neutrinos is different from that of neutrinos from oscillations. This reflects the structure of the $\nu_{\mu}$ spectrum from two-body $\pi_{\mu 2}$ and $K_{\mu 2}$ decays.

The NBB was used by experiments using bubble chambers $[108,173]$ or a fine-grained calorimeter [174] to search for neutrino oscillations. Their results are listed in Table 15. In particular, the fine-grained neutrino detector [174] shown in Fig. 51 was built to study weak NC interactions by deep-inelastic neutrino-nucleon scattering. The calorimeter is subdivided into modules, each made of flash chambers layered between plastic extrusions alternately filled with sand and steel shot. Proportional tube chambers provide the trigger for the flash chambers and give an independent measurement of the energy. 

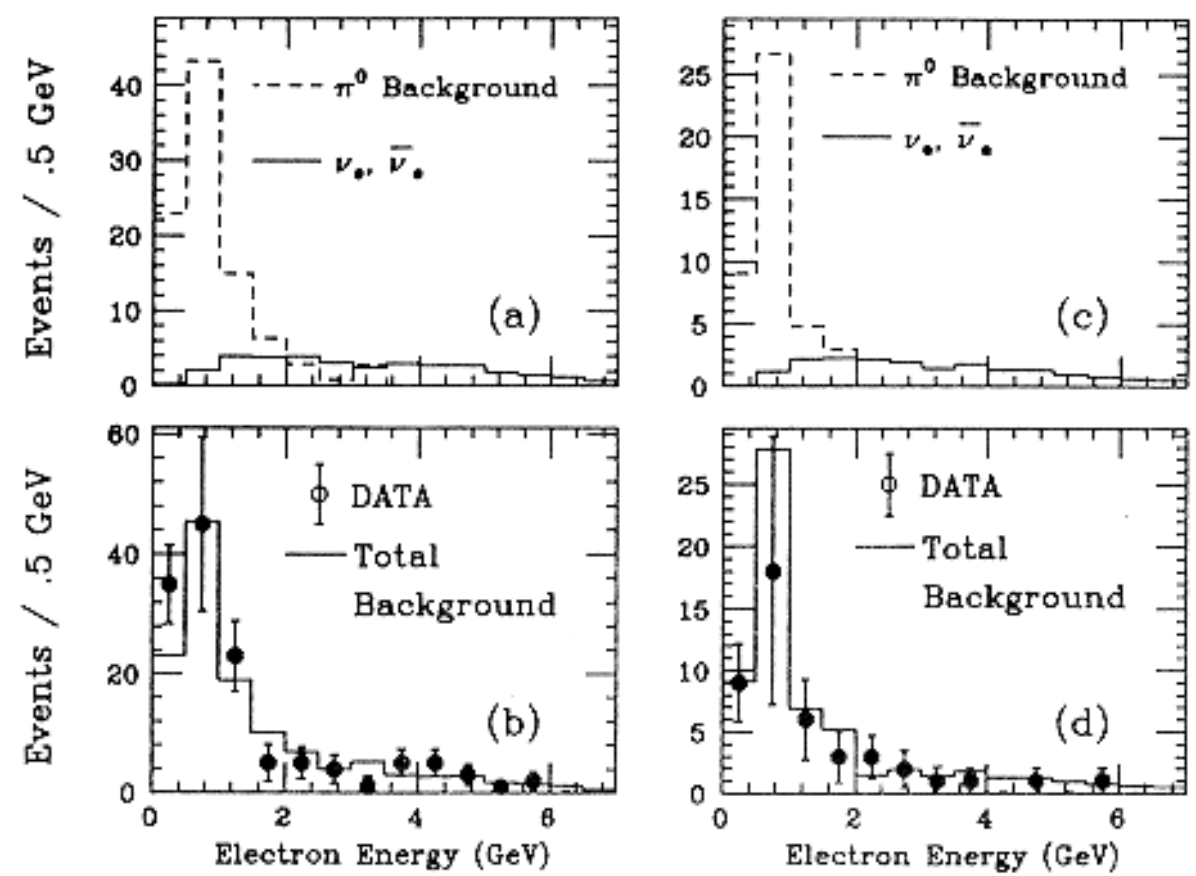

Figure 50: (a) The contributions to the background from $\nu_{\mu}$ induced $\pi^{0}$ events (dashed line) and from beam $\nu_{e}$ plus $\bar{\nu}_{e}$ (solid line) in the E776 experiment. (b) The electron energy spectrum for $\nu_{e}$ events. The solid line indicates the sum of the backgrounds. (a) and (b) refer to the horn at positive polarity, (c) and (d) to negative polarity. The error bars include the statistical error from the estimate of the $\pi^{0}$ contribution.

\begin{tabular}{||c|c|c|c|c|c||}
\hline Experiment & $\begin{array}{c}\bar{\nu}_{e} \leftrightarrow \bar{\nu}_{\mu} \\
\Delta m^{2} \mid \sin ^{2} 2 \theta\end{array}$ & $\begin{array}{c}\nu_{e} \leftrightarrow \nu_{\mu} \\
\Delta m^{2} \mid \sin ^{2} 2 \theta\end{array}$ & $\begin{array}{c}\bar{\nu}_{\mu} \leftrightarrow \bar{\nu}_{\tau} \\
\Delta m^{2} \mid \sin ^{2} 2 \theta\end{array}$ & $\begin{array}{c}\nu_{\mu} \leftrightarrow \nu_{\tau} \\
\Delta m^{2} \mid \sin ^{2} 2 \theta\end{array}$ & $\begin{array}{c}\nu_{e} \leftrightarrow \nu_{e} \\
\Delta m^{2} \mid \sin ^{2} 2 \theta\end{array}$ \\
\hline \hline Bubble Ch. [169] & & & & $3 \mid 0.05$ & \\
\hline Bubble Ch. [170] & & $0.6 \mid 0.006$ & & $3 \mid 0.06$ & $8 \mid 0.6$ \\
\hline Bubble Ch. [171] & & & $2.2 \mid 0.044$ & & \\
\hline Bubble Ch. NBB [108] & $2.4 \mid 0.013$ & & $7.4 \mid 0.088$ & & \\
\hline Bubble Ch. NBB [173] & & $2.2 \mid 0.011$ & & $6.3 \mid 0.088$ & $14.9 \mid 0.54$ \\
\hline Calo. NBB [174] & $3.1 \mid 0.04$ & $1.8 \mid 0.015$ & $6.5 \mid 0.15$ & $10.2 \mid 0.34$ & \\
\hline E564 [172] & & & & $4.5 \mid 0.06$ & \\
\hline \hline
\end{tabular}

Table 15: Summary of results obtained by Fermilab experiments searching for neutrino oscillations. $\Delta m^{2}$ values are given in $e V^{2}$. 
FMM NEUTRINO DETECTOR

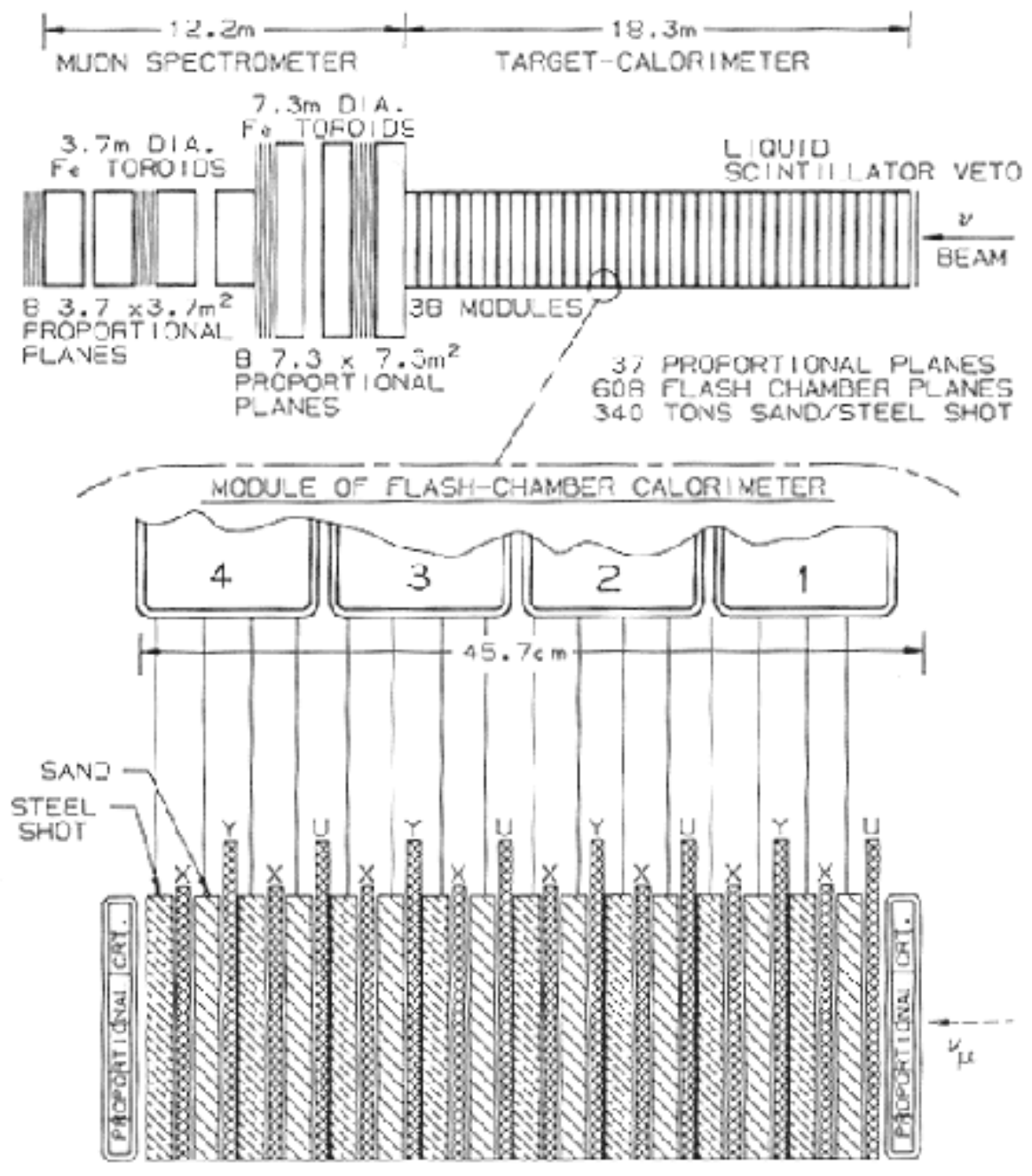

Figure 51: Schematic view of the fine-grained neutrino detector described in [174]. 
The high segmentation of the calorimeter allows the efficient detection of quasi-elastic neutrino interactions. Therefore, the experiment performed a search for neutrino oscillations taking advantage from the energy constraint of the NBB and of the simple kinematics of QE events.

$\nu_{\mu} \leftrightarrow \nu_{\tau}$ oscillations are searched for by looking at single lepton events. They consist of muons or electrons produced either by $\tau$ decay or by $\nu_{\mu, e} \mathrm{CC}$ interaction with an energy significantly smaller than the NBB neutrino energy. $\nu_{\mu} \leftrightarrow \nu_{e}$ oscillations can be studied by detecting single electromagnetic showers whose energy is consistent with the spectrum of the NBB. The results of the searches are summarised in Table 15. For information about the analysis method we refer to [174].

In the following, we describe in particular the Fermilab CCFR and E531 experiments.

\subsection{The CCFR experiment}

The CCFR neutrino detector [175] is schematically shown in Fig. 52. It was exposed to the Fermilab Tevatron Quadrupole Triplet neutrino beam [175]. Neutrinos are created by pions and kaons produced by $800 \mathrm{GeV}$ protons impinging on a production target. The beam has a $\nu / \bar{\nu}$ ratio equal to 2.5 and neutrino energies up to $600 \mathrm{GeV}$ with a $\nu_{e}+\bar{\nu}_{e}$ contamination of $2.3 \%$. The mean energies of $\nu_{\mu}$ and $\bar{\nu}_{\mu}$ are $185 \mathrm{GeV}$ and $143 \mathrm{GeV}$, respectively. The distance between target and detector is $1.4 \mathrm{~km}$.

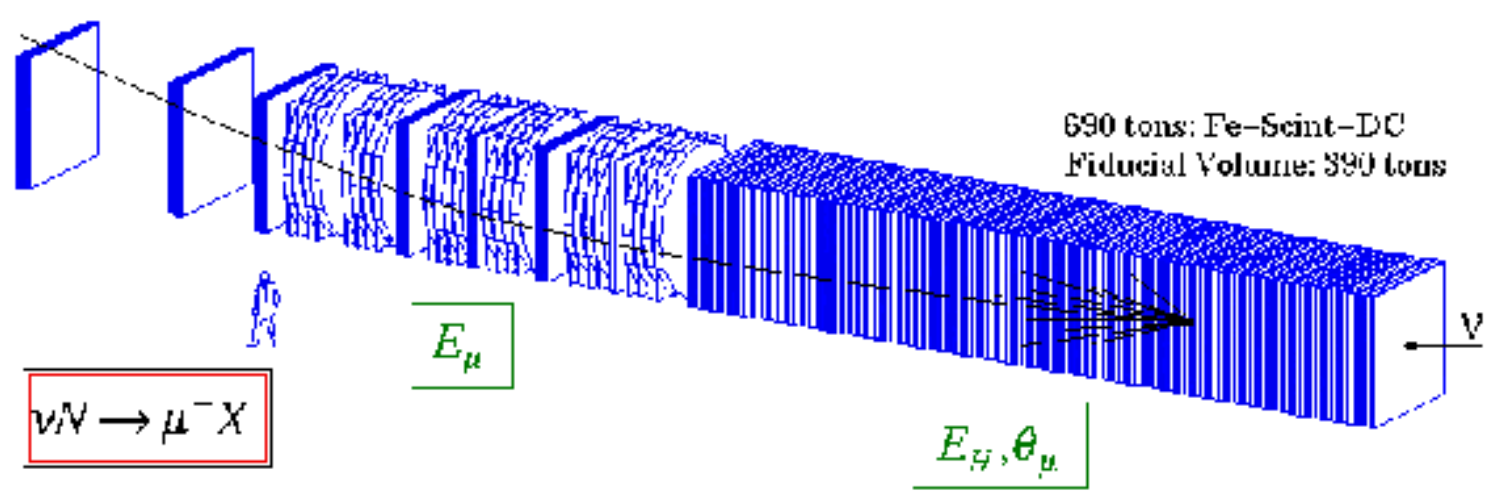

Figure 52: Schematic view of the CCFR detector.

The experimental setup consists of an iron target with muon tracking and a hadron shower calorimeter. The detector is backed by a muon spectrometer with three large toroidal iron-magnets instrumented with arrays of $3 \times 3 \mathrm{~m}^{2}$ drift chambers. The target calorimeter is made of 168 iron plates of $3 \mathrm{~m} \times 3 \mathrm{~m} \times 5.1 \mathrm{~cm}$ each. Liquid scintillator counters are placed every second plane and a drift chamber with two orthogonal planes every fourth plane. The total detector dimensions are $17.7 \times 3 \times 3 \mathrm{~m}^{3}$ for a mass of 690 ton and a mean density of $4.2 \mathrm{gr} / \mathrm{cm}^{3}$.

The experiment performed a search for $\nu_{\mu} \leftrightarrow \nu_{e}\left(\nu_{\tau}\right)$ oscillations [176]. CC interactions with a muon in the final state giving a signal in a large number of consecutive scintillator counters are classified as long events. Conversely, NC events are defined by an energy deposition over a limited number of planes. Neutrino interactions with energy release in 30 or fewer scintillator planes are classified as short events. 


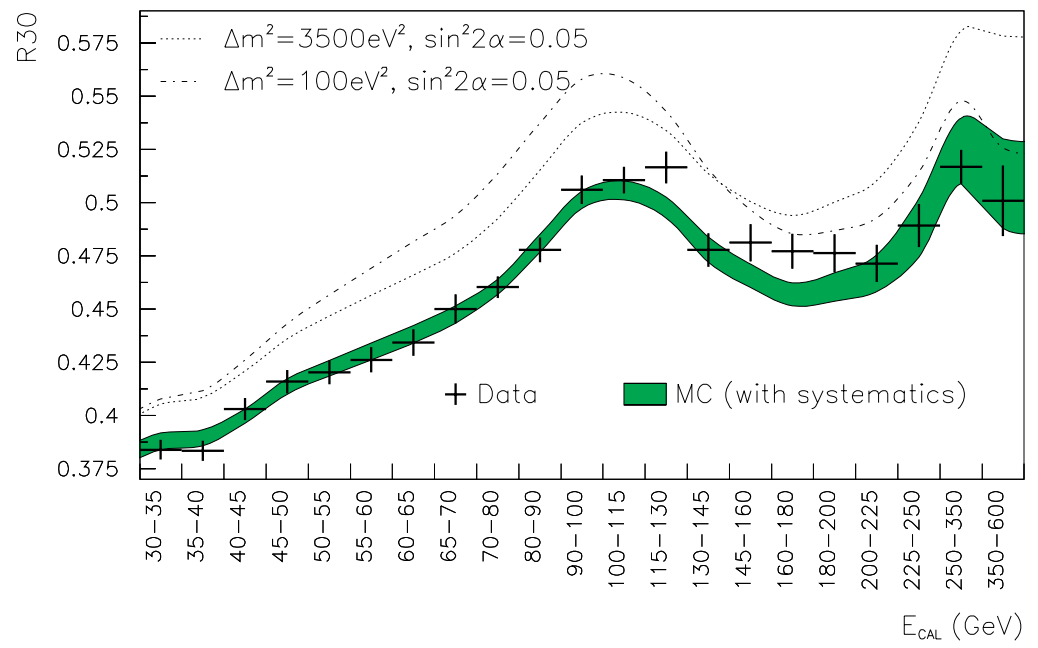

Figure 53: $R_{30}$ distribution for the CCFR experiment.

The ratio of short to long events $\left(R_{30}\right)$ strongly depends on the ratio of neutral to charged current interactions which, in turn, is a function of the electroweak mixing angle $\sin ^{2} 2 \theta_{W}$. The validity of the Standard Model is assumed in the analysis by using the $\sin ^{2} 2 \theta_{W}$ value as measured by other experiments. Possible deviations of the measured $R_{30}$ from the expectations are investigated. Any difference is assumed to be due to $\nu_{\mu} \leftrightarrow \nu_{e}$ or $\nu_{\mu} \leftrightarrow \nu_{\tau}$ oscillations.

Events are selected by requiring an energy deposition in the calorimeter $\left(E_{c a l}\right)$ above $20 \mathrm{GeV}$. The final event sample contains about 450000 interactions subdivided in 21 energy bins. The $R_{30}$ energy distribution relative to different values of $\Delta m^{2}$ is compared to the data (Fig. 53) together with the simulated distribution.

Four major sources of uncertainties are involved: the statistical error; the uncertainty in the effective charm-quark mass for $\mathrm{CC}$ charm production; the uncertainty in the incident flux of $\nu_{e}$; the uncertainty in the on-shell weak mixing angle as measured by other experiments. The data are fit by a $\chi^{2}$ function incorporating all statistical and systematic uncertainties and the possible effect of oscillations.

The result is consistent with the absence of $\nu_{\mu} \leftrightarrow \nu_{\tau, e}$ oscillations. The exclusion plot (90\% C.L.) relative to $\nu_{\mu} \leftrightarrow \nu_{\tau}$ oscillations is shown in Fig. 32. The best limit of $\sin ^{2} 2 \theta_{\mu \tau}<2.7 \times 10^{-3}$ is obtained for $\Delta m^{2}=50 \mathrm{eV}^{2}$. The limit for large $\Delta m^{2}$ is $\sin ^{2} 2 \theta_{\mu \tau}<8.1 \times 10^{-3} . \Delta m^{2}<1.4 e V^{2}$ is obtained for full mixing.

Concerning the $\nu_{\mu} \leftrightarrow \nu_{e}$ oscillation search, the best limit of $\sin ^{2} 2 \theta_{\mu e}<1.9 \times 10^{-3}$ corresponds to $\Delta m^{2}=350 \mathrm{eV}^{2}$. For large $\Delta m^{2}$ one obtains $\sin ^{2} 2 \theta_{\mu e}<3.8 \times 10^{-3} . \Delta m^{2}<1.6 e V^{2}$ is derived for full mixing.

An independent search for $\nu_{\mu} \leftrightarrow \nu_{e}$ oscillations is also carried out [177] in terms of the variable

$$
\eta_{3}=1-\frac{E_{1}+E_{2}+E_{3}}{E_{v i s}}
$$

$E_{i}$ is the energy deposited in the $i^{\text {th }}$ scintillation counter downstream of the interaction point and 
$E_{v i s}$ is the total energy released in the scintillation counters from the interaction vertex up to 5 counters beyond the shower end. The best limit of $\sin ^{2} 2 \theta_{\mu e}<1.1 \times 10^{-3}$ is obtained for $\Delta m^{2}=300 \mathrm{eV}^{2}$ and $\sin ^{2} 2 \theta_{\mu e}<1.8 \times 10^{-3}$ for large $\Delta m^{2}$. The limit of $\Delta m^{2}<1.6 e V^{2}$ is derived for $\sin ^{2} 2 \theta_{\mu e}=1$.

\subsection{The E531 experiment}

The E531 experiment [178] was originally proposed to study the properties of charmed particles and their production mechanism in neutrino interactions. For a description of the experiment we refer to [179]. A sensitive search for $\nu_{\mu} \leftrightarrow \nu_{\tau}$ oscillations could also be performed since the $\tau$ has a lifetime similar to that of charmed particles.

The neutrino beam is produced by $350 \mathrm{GeV}$ protons for the first exposure (for a total of $7.2 \times 10^{18} \mathrm{pot}$ ) and by $400 \mathrm{GeV}$ protons for the second $\left(6.8 \times 10^{18} \mathrm{pot}\right)$. The beam composition averaged over both runs is $92.3 \% \nu_{\mu}, 7.0 \% \bar{\nu}_{\mu}, 0.5 \% \nu_{e}$ and $0.2 \% \bar{\nu}_{e}$. The experimental target consists of nuclear emulsions where neutrinos interact and where short lived particles are precisely measured with micrometric accuracy. An electronic spectrometer detects the decay products. The hybrid detction technique adopted by E531 was further developed for the following CHORUS experiment, which has been described in Section 7.5.

The emulsion target consisted of 22.6 liters in the first run and of 30 liters in the second. It is made of modules composed of a series of sheets made up of a $300 \mu \mathrm{m}$ emulsion layer coated on both sides of polystyrene foils of $70 \mu \mathrm{m}$ thickness. Immediately downstream of the emulsion modules two large lucite sheets $800 \mu \mathrm{m}$ thick coated on both sides with $75 \mu \mathrm{m}$ emulsion layers are installed. These detectors (changeable emulsion sheets) allow to follow the particle tracks predicted by the electronic detectors and to ease their extrapolation into the emulsion target modules. The changeable sheets are replaced every two or three days of data taking in order to limit the total number of accumulated background-tracks.

Downstream of the target a magnet is equipped with high resolution drift chambers $\left(\sigma_{x} \sim 150 \mu \mathrm{m}\right.$, $\sigma_{\theta} \lesssim 1 \mathrm{mrad}$ ), which provide track predictions into the emulsions and tracking through the magnet. The momentum resolution is $\sigma_{p} / p=\sqrt{(0.014)^{2}+(0.004 p)^{2}}$. In addition, a time-of-flight detector made of two scintillator planes $2.7 \mathrm{~m}$ apart yields a time resolution $\sigma_{t} \lesssim 1 \mathrm{~ns}$. The setup is complemented by a lead glass array and a hadron calorimeter followed by a muon spectrometer. More details on the apparatus can be found in [180].

The event reconstruction starts with the search for tracks from neutrino interactions in the changeable sheets by means of predictions from the drift chambers. These detectors provide an accuracy of $320 \mu \mathrm{m}$ $(1 \sigma)$. An area of $\pm 1 \mathrm{~mm}^{2}$ of the changeable sheet is scanned around the predicted track impact point. The detected tracks are then followed into the emulsion target with a space resolution of $20 \mu m(1 \sigma)$ and an efficiency of about $95 \%$.

A total of 3886 neutrino interactions are located in the fiducial volume of the target. Possible $\nu_{\tau}$ interactions are identified by searching for $\tau$ decays. As in CHORUS their signature is given by the decay kink angle between the short lived $\tau$ track and its daughter particle track. Charged particle decays are found with a typical efficiency of $90-95 \%$ by following back tracks from the spectrometer into the emulsions.

Due to the presence of background processes, a further filter is applied to the selected events. Those with transverse momentum at the decay vertex lower than $125 \mathrm{MeV}$ are rejected in order to eliminate kinks faked by meson decays. A total of 104 candidates remains: 50 multi-prong and 54 one-prong. $\tau^{-}$are much more likely to be produced than $\tau^{+}$since the beam contains more than $90 \%$ neutrinos and $10 \%$ anti-neutrinos. For this reason only candidates consistent with a negative $\tau$ are retained: 25 events survive this cut. Those 
events with a prompt muon attached to the primary vertex are classified as $\nu_{\mu}$ CC interactions and rejected: 3 events are left. Finally, a $2.5 \mathrm{GeV}$ momentum cut is applied to the $\tau$ daughter candidates in order to remove background from re-interactions, scattering and decays of low momentum particles. None of the three candidates survives this cut.

The efficiency of the above selection is estimated to be $95 \%$ for the signal. The following 90\% C.L. limits are obtained for $\nu_{\mu, e} \leftrightarrow \nu_{\tau}$ oscillations

$$
\begin{array}{cc}
\sin ^{2} 2 \theta_{\mu \tau}<0.005 & \left(\text { large } \Delta m^{2}\right) \\
\Delta m_{\mu \tau}^{2}<0.9 e V^{2} & \left(\sin ^{2} 2 \theta_{\mu \tau}=1\right) \\
\sin ^{2} 2 \theta_{e \tau}<0.18 & \left(\text { large } \Delta m^{2}\right) \\
\Delta m_{e \tau}^{2}<9.0 e V^{2} & \left(\sin ^{2} 2 \theta_{e \tau}=1\right)
\end{array}
$$

The corresponding exclusion plot is shown in Fig. 32.

\section{Search for neutrino oscillations with low energy accelerators}

In this Section we report on results obtained by the experiments LSND at Los Alamos and KARMEN at the Rutherford Laboratory in searching for $\bar{\nu}_{\mu}\left(\nu_{\mu}\right) \leftrightarrow \bar{\nu}_{e}\left(\nu_{e}\right)$ oscillations. Both experiments use a neutrino beam produced by $800 \mathrm{MeV}$ protons. However, the two beam lines have different characteristics and are described separately. The two experiments have a partial overlap in the oscillation parameter regions which are investigated. LSND gives evidence for a neutrino oscillation signal.

\subsection{The LSND experiment}

Oscillation searches were performed with negative results [182-184] at the Los Alamos Meson Facility (LAMPF) before the claim of a positive signal by the LSND experiment [181]. The LSND (Liquid Scintillator Neutrino Detector) detector was designed to detect neutrinos and to search specifically for $\bar{\nu}_{\mu} \leftrightarrow \bar{\nu}_{e}$ and $\nu_{\mu} \leftrightarrow \nu_{e}$ oscillations. The $\bar{\nu}_{\mu} \leftrightarrow \bar{\nu}_{e}$ search is performed by looking at the appearance of $\bar{\nu}_{e}$ in the large flux of $\bar{\nu}_{\mu}$ from muon decay at rest (DAR). The $\nu_{\mu} \leftrightarrow \nu_{e}$ search is accomplished by looking at the appearance of $\nu_{e}$ interactions in a dominantly $\nu_{\mu}$ beam from pions which decay in flight (DIF).

The primary neutrino beam is obtained by sending $800 \mathrm{MeV}$ proton pulses with a frequency of $120 \mathrm{~Hz}$ onto a water target $30 \mathrm{~cm}$ long. Each pulse is approximately $600 \mu \mathrm{s}$ wide with a substructure consisting of pulses $0.25 \mathrm{~ns}$ wide at $201.25 \mathrm{M} \mathrm{Hz}$. Measurements of pion fluxes taken at different proton energies at LAMPF are used as input for the simulations $[185,186]$. Furthermore, the overall number of muon decays per incident proton was determined by an independent experiment.

Pions come to rest through the decay sequence

$$
\begin{array}{r}
\pi^{+} \rightarrow \mu^{+}+\nu_{\mu} \\
\mu^{+} \rightarrow e^{+}+\nu_{e}+\bar{\nu}_{\mu}
\end{array}
$$

which yields $\bar{\nu}_{\mu}$ with a maximum energy of $52.8 \mathrm{MeV}$. The energy dependence of the $\bar{\nu}_{\mu}$ and $\nu_{e}$ fluxes from DAR is known to $\pm 7 \%$ [20] and is shown in Fig. 54. The $\bar{\nu}_{\mu}$ flux integrated over the energy and 
averaged over the fiducial volume of the LSND detector amounts to $7.6 \times 10^{-10} \bar{\nu}_{\mu} /\left(\mathrm{cm}^{2}\right.$ proton $)$. The $\nu_{e}$ flux is identical to the $\bar{\nu}_{\mu}$ flux.

Due to the presence of a series of targets along the beam line, a small fraction of the neutrino flux is produced elsewhere than in the primary target. Simulations give $98.5 \%$ of the DAR flux originating from the primary target (located $30 \mathrm{~m}$ upstream of the detector) and $1.5 \%$ from the secondary ones (135 $\mathrm{m}$ and $110 \mathrm{~m}$ far from the detector, respectively) [187].

The chain starting with $\pi^{-}$may lead to an irreducible $\bar{\nu}_{e}$ background. However, three factors contribute to the suppression of $\bar{\nu}_{e}$. First, simulations show that in a water target the yield ratio $\pi^{-} / \pi^{+}$is $1 / 8$. Second, $\pi^{-}$coming to rest are captured via strong interaction before they can decay and produce $\bar{\nu}_{e}$. Only $5 \%$ of pions contributes to the $\bar{\nu}_{e}$ production. Third, $\mu^{-}$produced in the DIF of $\pi^{-}$come to rest in the beam stop before decaying. Most are captured from atomic orbit, a process which leads to $\nu_{\mu}$ but not to $\bar{\nu}_{e}$. This leaves only $12 \%$ of them to decay into $\bar{\nu}_{e}$. In conclusion, the relative yield compared with the positive polarity chain is estimated to be $\simeq(1 / 8) \times 0.05 \times 0.12=7.5 \times 10^{-4}$.

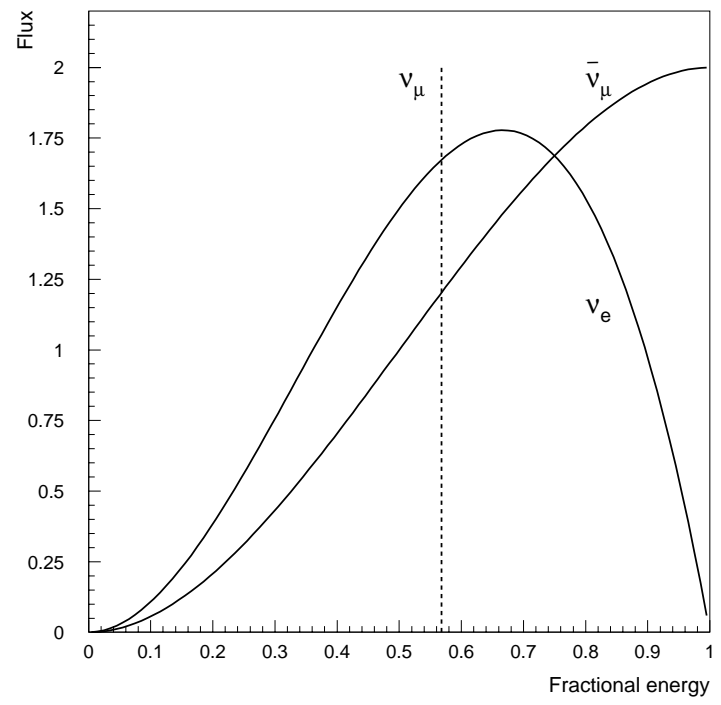

Figure 54: LSND experiment: neutrino spectra produced by the decay at rest of $\pi^{+}$and $\mu^{+}$.

The DIF flux is obtained by pions decaying in flight, $3.4 \%$ and $5 \%$ of $\pi^{+}$and $\pi^{-}$, respectively. The spectrum of the $\nu_{\mu}$ produced in flight extends up to $300 \mathrm{MeV}$ and is shown in Fig. 55. Due to the higher energy of those neutrinos and to the fact that the experiment aims at exploring $\Delta m^{2}$ values as small as $0.1 \mathrm{eV}^{2}$, the secondary targets provide a considerable contribution to the $\nu_{\mu} \leftrightarrow \nu_{e}$ oscillations because of the relatively long baseline.

The overall systematic error on the DIF fluxes has been estimated to be $15 \%$ [20]. A check of the flux calculation is performed by looking at the reaction $\nu_{\mu}{ }^{12} C \rightarrow \mu^{-12} N_{g s}$ which has a well known cross-section. This measurement confirms the calculated flux to within a $15 \%$ statistical error [188]. 

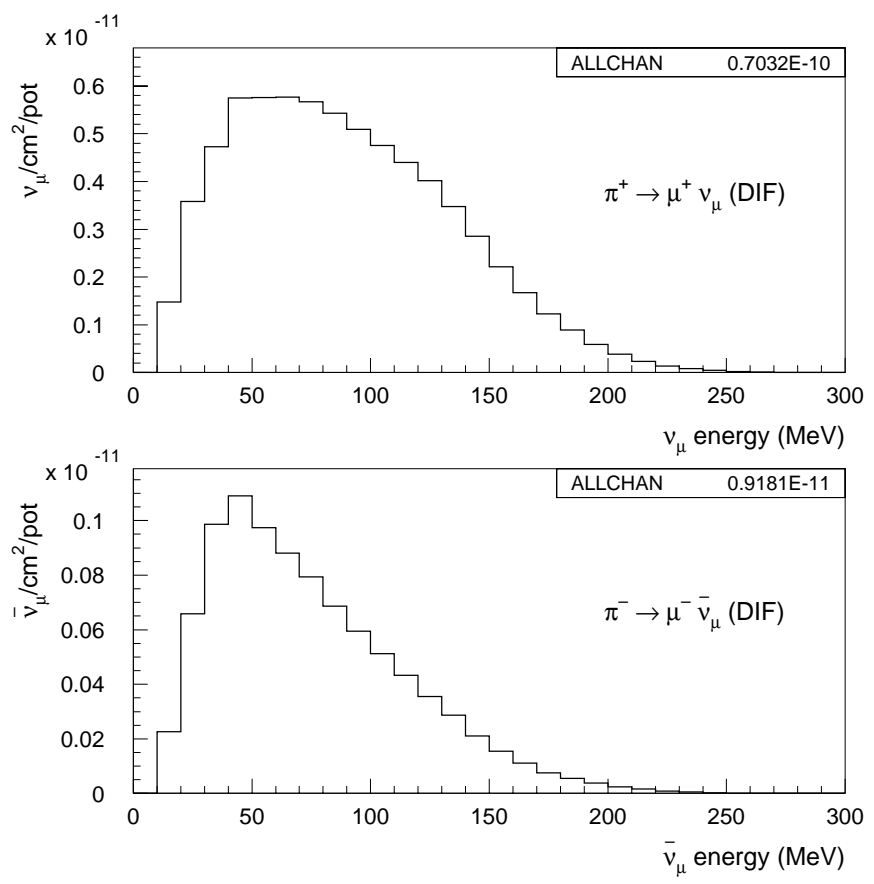

Figure 55: LSND experiment: neutrino spectra produced by the decay in flight of $\pi^{-}$and $\pi^{+}$. 


\subsubsection{The detector}
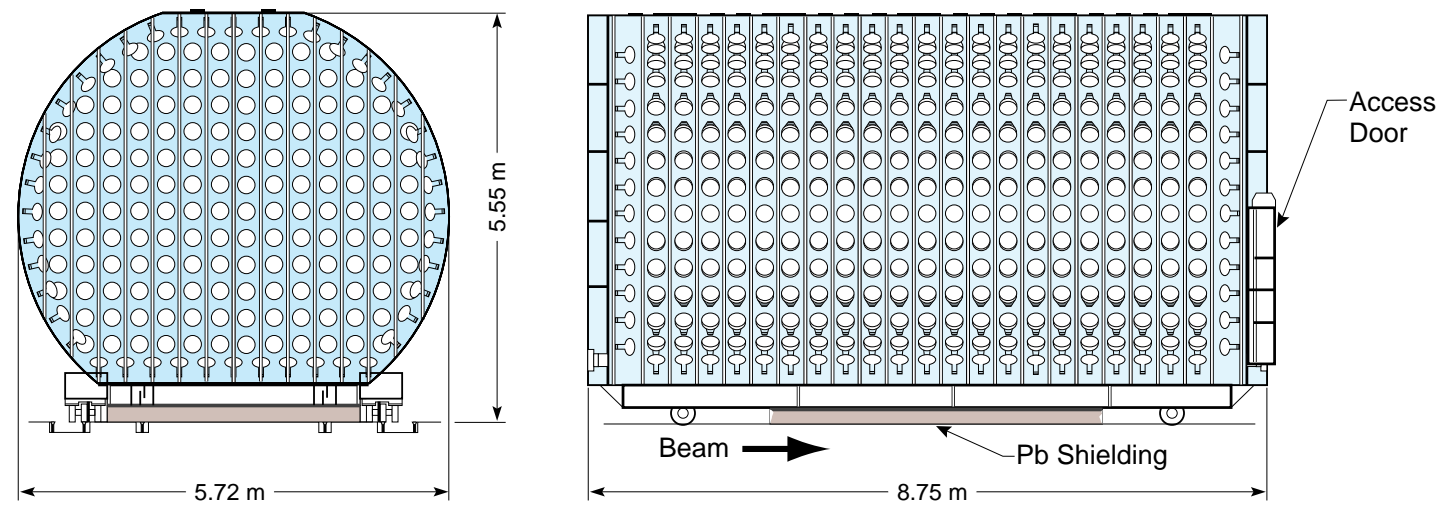

Figure 56: Front and side view of the LSND detector.

The LSND detector [189] is shown in Fig. 56. It consists of 167 ton of mineral oil $\left(\mathrm{CH}_{2}\right)$ doped with $6 \mathrm{~kg}$ of b-PBD scintillator. The concentration of b-PBD is measured to be $0.031 \mathrm{~g} / \mathrm{l}$. This low value allows the detection of both Cerenkov and scintillation light. The attenuation length is longer than $20 \mathrm{~m}$ for wavelengths above $400 \mathrm{~nm}$. The oil volume is viewed by $12208^{\prime \prime}$ PMTs which provide $25 \%$ photocathode coverage with uniform spacing.

The detector is contained in a steel tank roughly cylindrical in shape and located under $2 \mathrm{~kg} / \mathrm{cm}^{2}$ of steel overburden to shield it from cosmic rays. This shielding is sufficiently thick to stop the hadronic component of cosmic rays. A residual cosmic muon rate of $\sim 4 k H z$ is measured. Cosmic events are rejected by the active veto shielding surrounding the whole detector except at the bottom and at the downstream parts. The shielding consists of liquid scintillator viewed by $2925^{\prime \prime}$ PMTs. Muons penetrating through the veto and stopping in the detector represent a source of electrons used for calibration purposes.

An estimate of the LSND event vertex reconstruction capability can be obtained by studying $\nu_{\mu} C \rightarrow \mu X$ events. Both electrons and stopping muons are reconstructed in position and the distance between the muon and the beginning of the electron track are compared. Simulations well agree with the experimental data and indicate a vertex resolution of $20 \mathrm{~cm}$ [189].

The angular resolution in the LSND detector is determined by measuring at the angular distribution of Michel electrons. Due to multiple scattering, the angular resolution is limited to $12^{\circ}$ for $50 \mathrm{MeV}$ electrons. An independent check can be performed by looking at the $\cos \theta$ distribution for electrons produced in neutrino-electron scattering. The results are consistent with an angular resolution of $12^{\circ}$ for electrons with energy above $20 \mathrm{MeV}$ [189].

The detector energy resolution is evaluated by using Michel electrons. It amounts to $7.7 \%$ at $52.8 \mathrm{MeV}$ (end point of the $\mu^{+}$decay spectrum). The linearity of response for electron energies in the interesting range is measured by studying $\nu_{e}^{12} \mathrm{C}$ scattering (with electron end point of $35.4 \mathrm{MeV}$ ) and the $\beta$ decay spectrum from ${ }^{12} N_{g s}$ (end point of $16 \mathrm{MeV}$ ). The calibration for energies above $52.8 \mathrm{MeV}$, relevant for the DIF analysis, is obtained by extrapolating the calibration performed at lower energies. 
Particle identification capability in LSND is studied by selecting neutron events induced by cosmic rays and by analysing Michel electron events. A 96\% efficiency for selecting electrons above $36 \mathrm{MeV}$ and a rejection of $10^{-3}$ against neutrons are achieved.

\subsection{2 $\bar{\nu}_{\mu} \leftrightarrow \bar{\nu}_{e}$ oscillation search from muonic decay at rest}

LSND detects $\bar{\nu}_{e}$ through the reaction $\bar{\nu}_{e} p \rightarrow e^{+} n$ followed by the neutron-capture reaction $n p \rightarrow$ $d \gamma(2.2 \mathrm{MeV})$. Hence, the signature of neutrino oscillations then consists of an electron signal followed by a $2.2 \mathrm{MeV}$ photon correlated in time and position to the positron.

As pointed out in Section 10.1 the $\bar{\nu}_{e}$ contamination from DAR is very small $\left(\sim 10^{-4}\right)$. Nevertheless, since the detector is not able to distinguish electrons from positrons, it is necessary to deal with the $\nu_{e}$ from DAR produced one-by-one with the desired $\bar{\nu}_{\mu}$. Details about the muonic decay at rest analysis can be found in $[21,22]$.

In LSND the detection of $\nu_{e}$ is dominated by the CC interaction $\nu_{e}^{12} C \rightarrow{ }^{12} N e^{-}$with an electron energy $E_{e}<36 \mathrm{MeV}$. Moreover, it is possible to produce a photon correlated with the electron signal through the reaction $\nu_{e}^{12} C \rightarrow e^{-} \gamma^{11} N$ if $E_{e}<20 \mathrm{MeV}$. In order to reduce as much as possible the background from the above processes only events with an electron with $E_{e}>36 \mathrm{MeV}$ are retained. An upper energy cut of $60 \mathrm{MeV}$ is also applied to allow the $\bar{\nu}_{\mu}$ end point to be smeared by the energy resolution.

The separation of correlated neutron-capture photons from accidental signals is performed by using an approximate likelihood ratio $R \equiv \mathcal{L}_{c} / \mathcal{L}_{a}$ for the correlated $\left(\mathcal{L}_{c}\right)$ and accidental hypotheses $\left(\mathcal{L}_{a}\right)$. Each likelihood is a function of the time interval between the photon and the primary event $(1 \mathrm{~ms})$, of the number of PMT hits (from 21 to 50) and of the distance between the detected photon and the primary event $(2.5 \mathrm{~m})$.

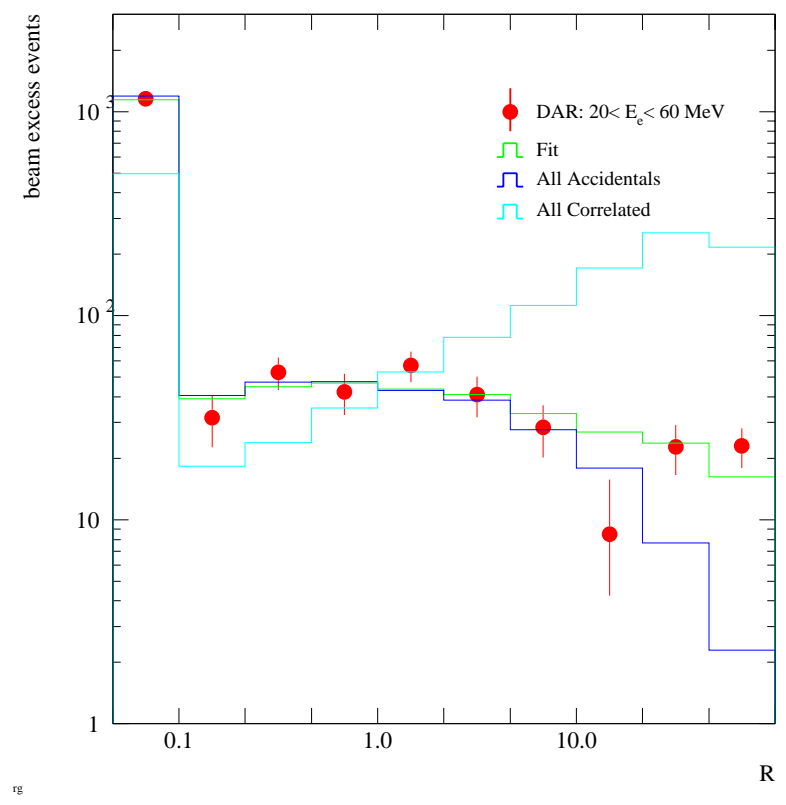

Figure 57: LSND likelihood ratio distribution for events with a photon correlated or uncorrelated to the primary event. 


\begin{tabular}{||c|c|c|c||}
\hline Selection A & Efficiency & Selection B & Efficiency \\
\hline \hline Particle ID & $0.77 \pm 0.02$ & Particle ID & $0.84 \pm 0.01$ \\
\hline$<2$ Veto Hits & $0.84 \pm 0.02$ & $<4$ Veto Hits & $0.98 \pm 0.01$ \\
\hline$\Delta t_{p}>40 \mu s$ & $0.50 \pm 0.02$ & $\Delta t_{p}>20 \mu s, 34 \mu s$ & $0.68 \pm 0.02$ \\
\hline DAQ dead-time & $0.97 \pm 0.01$ & DAQ dead-time & $0.97 \pm 0.01$ \\
\hline $35 \mathrm{~cm}$ fiducial volume & $0.85 \pm 0.05$ & $35 \mathrm{~cm}$ fiducial volume & $0.85 \pm 0.05$ \\
\hline No events within $8 \mu s$ & $0.99 \pm 0.01$ & No events within $8 \mu s$ & $0.99 \pm 0.01$ \\
\hline$<3$ associated $\gamma \mathrm{s}$ & $0.99 \pm 0.01$ & $<2$ associated $\gamma \mathrm{s}$ & $0.94 \pm 0.01$ \\
\hline- & - & $S>0.5$ & $0.87 \pm 0.02$ \\
\hline \hline Total & $0.26 \pm 0.02$ & Total & $0.37 \pm 0.03$ \\
\hline
\end{tabular}

Table 16: LSND DAR analysis: selection criteria and corresponding efficiencies for two independent positron selection criteria (selections $A$ and $B$ in the text).

The $R$ distribution for events with a photon correlated or uncorrelated to the primary event is shown in Fig. 57. Events without a photon have by definition $R=0$. Uncorrelated events are concentrated at low $R$ values. The probability that an accidental $\gamma$ satisfies $R>30(1.5)$ is $0.6 \%(9.0 \%)$, while the efficiency for a correlated $\gamma$ is $23 \%$ (58\%). The efficiency of this cut has been checked by using Michel electron events.

Two independent analyses are conducted to select positron events. They are called selection $A$ and $B$. The main difference between them is that selection $A$ exploits improved insight into the nature of the beam-off backgrounds to further reduce them while relaxing other criteria. The selection criteria and the corresponding efficiencies for selection $A$ and $B$ are listed in Table 16.

In the first step one requires the primary particle to be consistent with a positron. The number of veto shielding hits associated with the event is required to be less than 2 for selection $A$ and less than 4 for selection $B$ in order to reduce cosmic-ray background. The time interval $\Delta t_{p}$ from the previous event must be larger than $40 \mu s$ for selection $A$ and $20 \mu s$ for selection $B$. This eliminates Michel electrons.

Selection $B$ implies additional cuts. The first requires that signals between $20 \mu s$ and $34 \mu s$ before the event trigger have to be uncorrelated to the positron. This is obtained by asking fewer than 50 PMT hits and reconstructed position further than $2 \mathrm{~m}$ from the positron position. The positron has to be at least $35 \mathrm{~cm}$ away from the PMTs. This cut selects positrons produced in a region of the tank in which the energy and the particle identification efficiency vary smoothly.

The timing for any subsequent triggered event is required to be $>8 \mu s$ to remove events originating from muon decays. In order to reduce cosmic-ray induced neutrons, the number of associated $\gamma_{\mathrm{s}}$ with $R>1.5$ has to be less than three for selection $A$ and less than two for selection $B$.

Selection $B$ includes a cut on the likelihood ratio $S \equiv \mathcal{L}_{\text {on }} / \mathcal{L}_{\text {off }}$ for further background reduction. The likelihoods $\mathcal{L}_{\text {on }}$ and $\mathcal{L}_{\text {off }}$ are calculated for beam-on background events and for beam-off signal events, respectively. The variables used to build $\mathcal{L}_{\text {on }}$ and $\mathcal{L}_{\text {off }}$ are the distribution of angles between the $e^{+}$ direction and its position vector relative to the tank center (background events tend to head inwards) and the distribution of the veto hits (cosmic-ray events tend to have more). One can eliminate $33 \%$ of the background with a signal efficiency of $87 \%$ by requiring $S>0.5$.

The backgrounds to the $\bar{\nu}_{\mu} \leftrightarrow \bar{\nu}_{e}$ signal can be subdivided into two categories 


\begin{tabular}{||c|c|c|c|c||}
\hline Data sample & Fitted excess & Neutrino background & Total excess & Oscill. probability \\
\hline \hline $1993-1995$ & $63.5 \pm 20.0$ & $12.5 \pm 2.9$ & $51.0 \pm 20.2$ & $(3.1 \pm 1.2 \pm 0.5) \times 10^{-3}$ \\
\hline $1996-1997$ & $35.1 \pm 14.7$ & $4.8 \pm 1.1$ & $30.3 \pm 14.8$ & $(3.2 \pm 1.5 \pm 0.5) \times 10^{-3}$ \\
\hline $1993-1998$ & $111.8 \pm 25.6$ & $20.9 \pm 4.8$ & $90.9 \pm 26.1$ & $(3.3 \pm 0.9 \pm 0.5) \times 10^{-3}$ \\
\hline \hline
\end{tabular}

Table 17: LSND excess of events over background and the corresponding oscillation probabilities from fits to the $R$ distributions for the three running periods 1993-1995, 1996-1997 and 1993-1998.

- beam-off events induced by cosmic rays. The background to beam-on events is 0.07 times the number of beam-off events which pass the same selection criteria;

- beam-related events with a neutrino interaction and either a correlated neutron or an accidental $\gamma$.

Six months of additional data were collected in 1996 and 1997 using a different beam stopper [190]. The water target was replaced with a close-packed tungsten target. The neutrino beam so obtained is substantially different from the one used previously, hence allowing for a systematic check of the earlier results. With the new configuration the pion DIF flux is reduced to $1 / 2$ of the original one and the muon DAR to $2 / 3$. The result obtained by only using this sample (Table 17 ) yields a significant beam-related excess of events consistent with the former result, although statistically less significant.

Kolmogorov tests have been performed in order to look for unexpected concentration of events in position, energy or time. None of the tests indicates any inconsistency. The oscillation probability is obtained through a fit to the overall $R$ distribution for events satisfying selection $B$ in the full range $20<E_{e}<60 \mathrm{MeV}$.

After subtraction of the neutrino background with a recoil neutron, the full 1993 - 1998 data sample shows a positive oscillation signal with an excess of $90.9 \pm 26.1$ events with $R>0$ in the region $36<E_{e}<$ $60 \mathrm{MeV}$. The oscillation probabilities derived through a fit to the $R$ distributions for the different event samples are shown in Table 17. The probability for the whole data set is $(3.3 \pm 0.9 \pm 0.5) \times 10^{-3}$. The corresponding allowed region of the plane $\Delta m^{2}-\sin ^{2} 2 \theta$ is shown in Fig. 58 .

\subsection{3 $\nu_{\mu} \leftrightarrow \nu_{e}$ oscillation search from pion decay in flight}

A candidate $\nu_{\mu} \leftrightarrow \nu_{e}$ event from a DIF $\nu_{\mu}$ consists of a single and isolated electron with energy in the range $60<E_{e}<200 \mathrm{MeV}$ produced in the reaction $\nu_{e} C \rightarrow e^{-} X$. The lower bound is set just above the Michel electron end-point to reduce cosmic-ray background. The upper bound corresponds to the region in which beam-off background starts to be important and the oscillation signal small.

The main difficulty of the DIF analysis is that it only relies on the electron particle identification and, contrary to the DAR data, there are no control samples to check the efficiency with the exception of the reaction $\nu_{\mu}^{12} C \rightarrow \mu^{-12} N_{g s}$.

A first attempt in selecting electron tracks can be performed using the same criteria developed for the DAR analysis. The electron efficiency is very high $(0.98 \pm 0.02)$ while the background rejection capability poor. Therefore, further electron particle identification (PID) criteria and cuts to reject the background have to be developed. Without entering into details which can be found in [19,20], the main purpose of the electron PID is to select events consistent with an oscillation signal while rejecting neutrons and photons background induced by cosmic rays. 


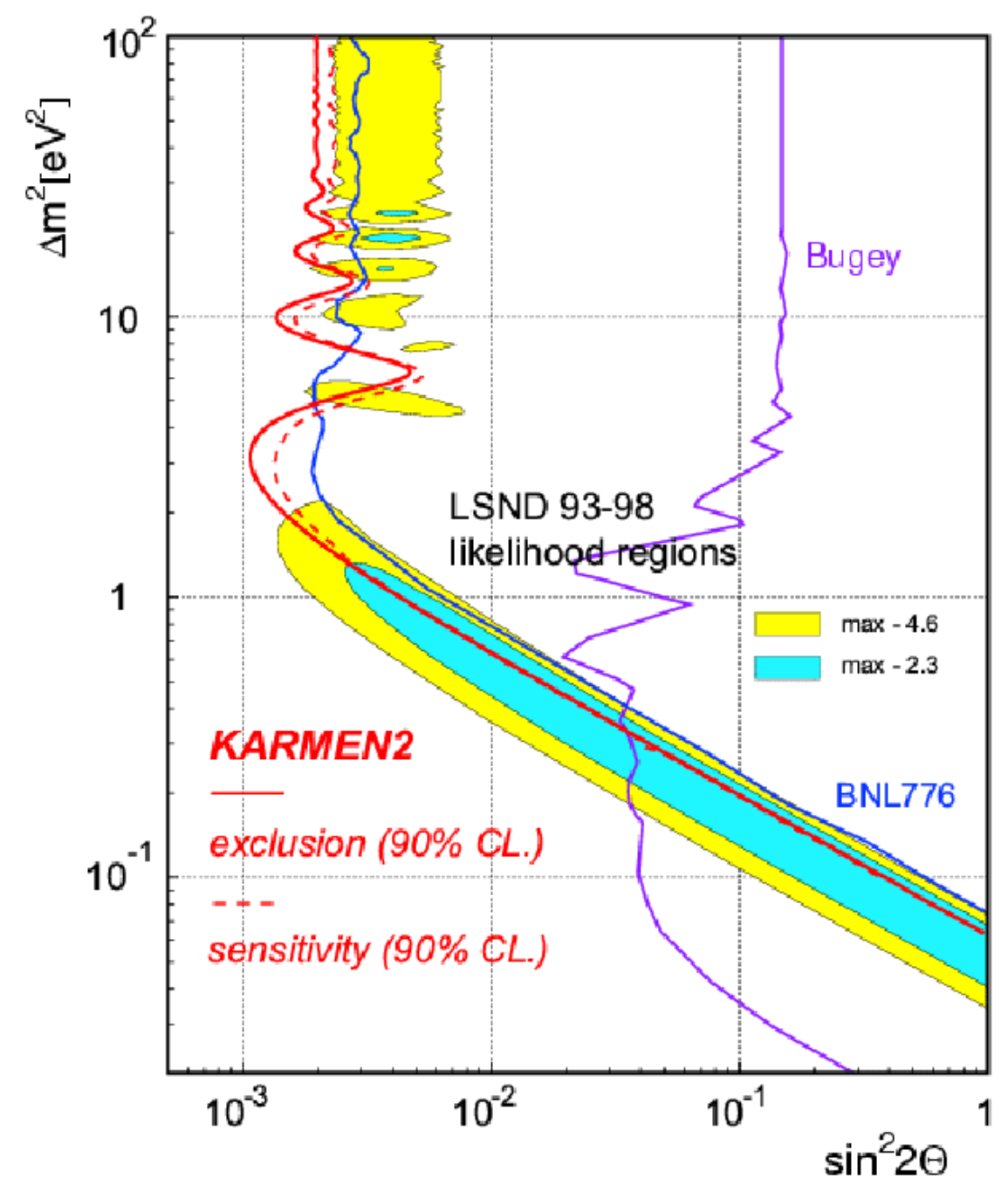

Figure 58: Allowed region by the LSND DAR analysis. It is assumed that the excess of $\bar{\nu}_{e}$ events is due to neutrino oscillations. Excluded regions by other experiments are also shown. 


\begin{tabular}{||c|c|c|c|c|c|c||}
\hline Data sample & Beam On/Off & BUB & BRB & Total excess & Efficiency & Oscill. prob. \\
\hline \hline Selection $A$ & $23 / 114$ & $8.0 \pm 0.7$ & $4.5 \pm 0.9$ & $10.5 \pm 4.9$ & $8.4 \%$ & $(2.9 \pm 1.4) \times 10^{-3}$ \\
\hline Selection $B$ & $25 / 92$ & $6.4 \pm 0.7$ & $8.5 \pm 1.7$ & $10.1 \pm 5.3$ & $13.8 \%$ & $(1.7 \pm 0.9) \times 10^{-3}$ \\
\hline$A$ AND $B$ & $8 / 31$ & $2.2 \pm 0.3$ & $3.1 \pm 0.6$ & $2.7 \pm 2.9$ & $5.5 \%$ & $(1.1 \pm 1.2) \times 10^{-3}$ \\
\hline$A$ OR $B$ & $40 / 175$ & $12.3 \pm 0.9$ & $9.6 \pm 1.9$ & $18.1 \pm 6.6 \pm 3.5$ & $16.5 \%$ & $(2.6 \pm 1.0) \times 10^{-3}$ \\
\hline \hline
\end{tabular}

Table 18: Comparison of LSND results for DIF analysis: selections $A, B$, their AND and their OR. BUB stands for the beam-unrelated background from cosmic rays.

Particle identification relies on differences in the timing characteristics of the light components produced in the event: scintillation and Cerenkov light both direct and re-scattered. As for the DAR analysis, two completely independent analyses are carried out after event selection. Since they are based on different reconstruction programs, cuts and systematics errors, their results may in principle differ. The results (selection $A$ and selection $B$ ) are summarised in Table 18. The final DIF sample is obtained by considering the OR of the two analyses.

The main sources of beam-related background (BRB) in the DIF search are

- the intrinsic $\nu_{e}$ contamination of the beam. This background is evaluated by using simulated neutrino fluxes and the well known $\nu_{e} C$ cross-section [191,192];

- the $\nu_{\mu} e$ elastic scattering from $\nu_{\mu}$ DIF flux. This is greatly reduced by requiring $\cos \theta_{e}<0.8$, with $\theta_{e}$ being the angle between the reconstructed electron direction and the incident neutrino beam direction;

- the reaction $\nu_{\mu} C \rightarrow \nu_{\mu} C \pi^{0}$ with $\pi^{0}$ decay and subsequent $\gamma$ conversion which fakes a primary electron [193].

The numbers of BRB events expected for the two analyses are given in Table 18. The probability that the number of background events (BRB+BUB) (12.5/14.9) fluctuates up to the observed beam-on value $(23 / 25)$ is $7 \times 10^{-3}$ and $1.6 \times 10^{-2}$ for selection $A$ and $B$, respectively. The interpretation of these results in terms of neutrino oscillations is illustrated in Fig. 59. The result is consistent with the one obtained with the DAR analysis.

\subsection{The KARMEN experiment}

The Karlsruhe Rutherford Medium Energy Neutrino (KARMEN) experiment [194] was originally designed to study neutrino nucleus interactions and to search for physics beyond the Standard Model. Physics goals were, in particular, neutrino oscillations, lepton number violating decays of pions and muons as well as the $\mathrm{V}$-A structure of the $\mu^{+}$decay. Here we present the results obtained in the appearance oscillation channels $\nu_{\mu} \leftrightarrow \nu_{e}$ and $\bar{\nu}_{\mu} \leftrightarrow \bar{\nu}_{e}$ based on data taken from 1990 to 1995 with the KARMEN1 and then with the upgraded KARMEN2 detectors.

The KARMEN experiment is performed at the neutron spallation facility ISIS at the Rutherford Appleton Laboratory. Pions are created in the interaction of $800 \mathrm{MeV}$ protons onto a heavy target $\left(\mathrm{Ta}-\mathrm{D}_{2} \mathrm{O}\right)$ and $\pi^{-}$are absorbed by nuclei. Neutrinos are produced by $\pi^{+}$DAR. A small contamination of $\bar{\nu}_{e}$ $\left(\bar{\nu}_{e} / \nu_{e}=6.2 \times 10^{-4}[187]\right)$ originates from $\pi^{-}$decays in flight and from the subsequent $\mu^{-} \mathrm{DAR}$. Off-line cuts allow to reduce the $\bar{\nu}_{e}$ background by about a factor two. 


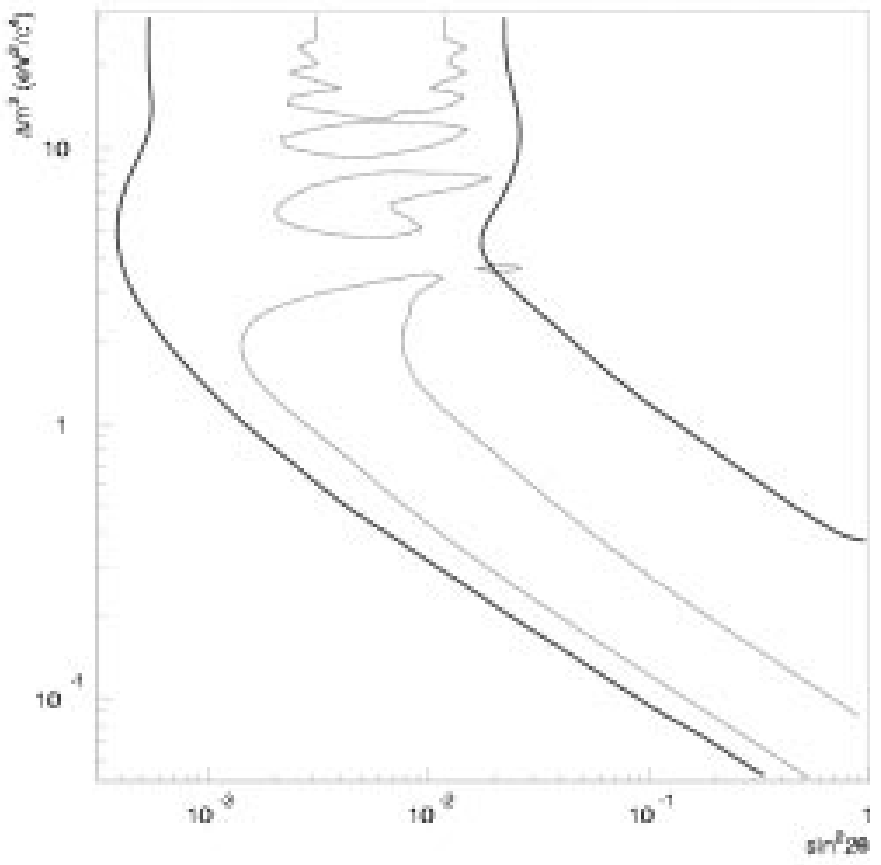

Figure 59: Region allowed by the LSND DIF analysis if the excess of $\nu_{e}$ is due to neutrino oscillations (thick line). The region allowed by the DAR analysis is also shown (thin line). 
The KARMEN neutrino spectrum is similar to that of LSND. The KARMEN experiment, however, profits from the peculiar time structure of the beam. Two parabolic proton pulses of $100 \mathrm{~ns}$ base width with a gap of $225 \mathrm{~ns}$ are produced with a repetition rate of $50 \mathrm{~Hz}$ (Fig. 60). The different mean lifetimes of the $\pi(26 n s)$ and of the $\mu(2.2 \mu s)$ ensure a clear separation in time of the $\nu_{\mu}$ burst from the following $\nu_{e}$ and $\bar{\nu}_{\mu}$. Furthermore, due to the heavy target, the $\nu$ production region is essentially limited to a region $\pm 5 \mathrm{~cm}$ radial to the proton beam and $\pm 10 \mathrm{~cm}$ along the beam axis. This allows an excellent resolution in the measurement of the baseline $(\langle L\rangle=17.6 \mathrm{~m}$ and $\Delta L / L<1 \%)$.
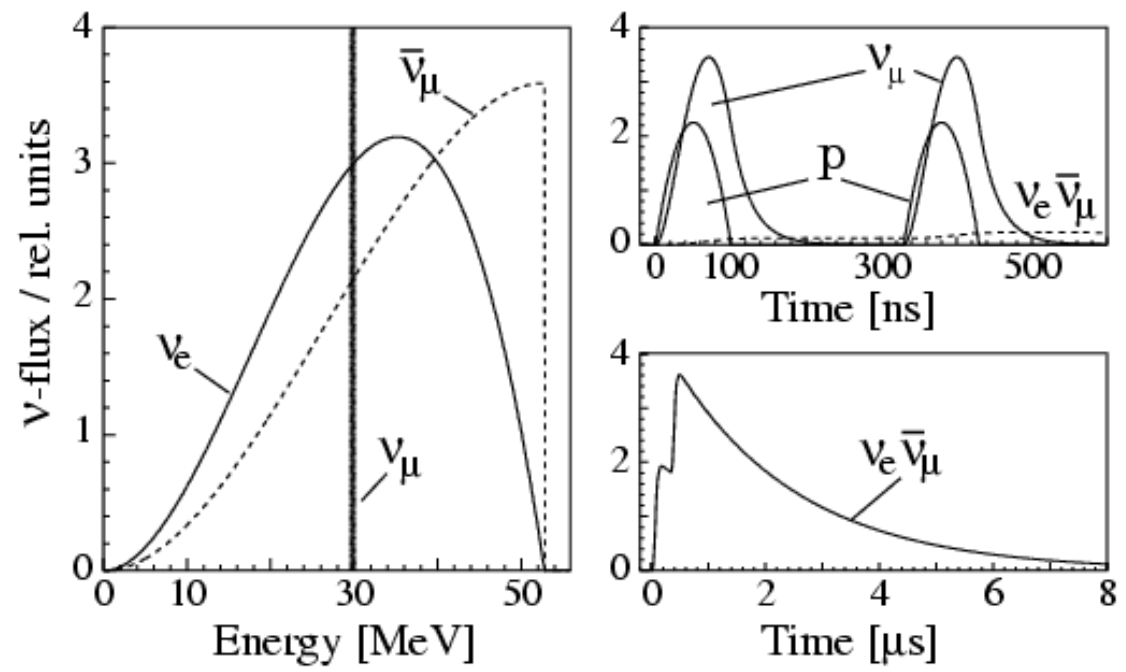

Figure 60: Neutrino energy spectra and time distribution of the ISIS beam.

The KARMEN1 detector [195] consists of a 56 ton liquid scintillator calorimeter segmented into cells and of a veto made of 7000 ton of steel instrumented with layers of active counters. The experimental setup is schematically shown in Fig. 61.

The granularity of each cell is $0.18 \times 0.18 \times 3.53 \mathrm{~m}^{3}$. The event position is determined by the coordinates of the cell and by the time difference of the PMT signals at either ends of the cell. For typical energies of $30 \mathrm{MeV}$ the position resolution is $\pm 6 \mathrm{~cm}$. Due to the good optical properties of the liquid scintillator and to the large calorimeter active volume of $96 \%$, the excellent energy resolution of $\sigma_{E}=11.5 \% / \sqrt{E(\mathrm{MeV})}$ is achieved.

The calorimeter is covered by two layers of counters (the outer veto1 detector in Fig. 61). The central detector and the inner veto counters are segmented by acrylic walls with an air gap allowing efficient light transport via total reflection of the scintillation light at the module walls.

The aim of the veto is to provide shielding against neutrons produced by ISIS and to suppress the hadronic component of cosmic rays as well as cosmic muons. The efficiency of the veto system amounts to $99.9 \%$.

In order to maximise the detection of thermal neutrons, $\mathrm{Gd}_{2} \mathrm{O}_{3}$ coated paper is inserted within the cell walls. The cross-section for the reaction $(G d(n, \gamma))$ is $\sigma \sim 49000$ barn. The electronics of the detector is synchronised to the ISIS extraction timing with an accuracy of $\pm 2 \mathrm{~ns}$, sufficient to sample the time structure of the neutrino beam. The experiment took data from 1990 to 1995 . 


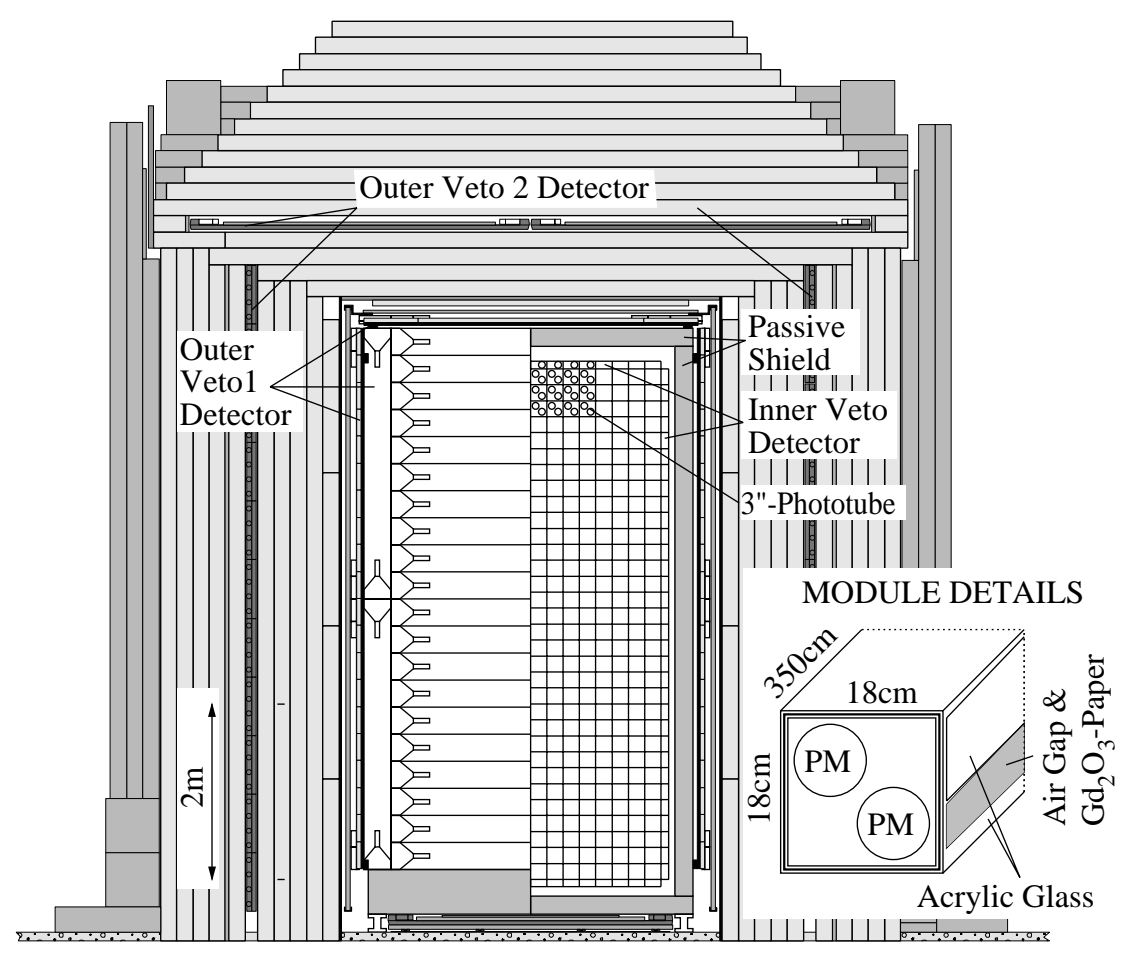

Figure 61: The KARMEN calorimeter in the 7000 ton shielding blockhouse.

The experiment sensitivity is limited by the background due to high energy neutrons produced in deepinelastic interactions of cosmic-ray muons in the steel surrounding the detector. Simulations show that, in order to reduce this background, the passive shield has to be instrumented for muon detection. The KARMEN1 upgrade mainly consisted in the instrumentation of the passive shield. An additional veto layer was embedded in the walls and the roof of the passive shield (the outer veto2 detector in Fig. 61). This reduces by $98 \%$ the background induced by neutrons. The KARMEN2 detector started data taking in February 1997.

\subsubsection{Oscillation search with the KARMEN1 detector}

The search for $\nu_{\mu} \leftrightarrow \nu_{e}$ oscillations proceeds by looking for $\nu_{e}$ coming from mono-energetic $\nu_{\mu}$ in the beam [195-197]. Muon neutrinos are characterised by a prompt time signature due to the time structure of the ISIS beam. The slow $\nu_{e}$ component of the beam can be used to check the detection efficiency. $\nu_{e}$ interactions are identified through the reaction $\nu_{e}^{12} C \rightarrow e^{-12} N_{g s}$ followed by the $\beta$-decay ${ }^{12} N_{g s} \rightarrow{ }^{12} C+e^{+}+\nu_{e}$.

Two events are observed fulfilling the selection criteria. The total estimated background is $N_{b g}=$ $2.26 \pm 0.30$ events. Therefore, no evidence for $\nu_{\mu} \leftrightarrow \nu_{e}$ oscillations is found and the $90 \%$ C.L. upper limit $P\left(\nu_{\mu} \leftrightarrow \nu_{e}\right)<2.0 \times 10^{-2}$ is set. The sensitivity to this oscillation channel, although nearly background-free, is limited by the relative smallness of the ISIS primary target.

The $\bar{\nu}_{\mu} \leftrightarrow \bar{\nu}_{e}$ channel is the most sensitive to neutrino oscillations. The main reasons are the small $\bar{\nu}_{e}$ contamination of the beam and the fact that the cross-section for the $\bar{\nu}_{e} p \rightarrow e^{+} n$ reaction employed to detect $\bar{\nu}_{e}$ is 20 times larger than for $\nu_{e}^{12} C \rightarrow e^{-12} N_{g s}$. This allows to gather enough statistics and probe 


\begin{tabular}{||c|c||}
\hline Reduction cut & Efficiency \\
\hline \hline No events within $20 \mu s$ before a prompt candidate & 0.912 \\
\hline Prompt energy: $16 \leq E_{p r} \leq 50 \mathrm{MeV}$ & 0.611 \\
& \\
Prompt fiducial volume $(0.7$ of central detector $)$ & \\
\hline Prompt time relative to ISIS: $0.6 \leq t_{p r} \leq 10.6 \mu s$ & 0.842 \\
\hline Neutron detection & 0.282 \\
\hline \hline Total efficiency for $e^{+}-n\left(\Delta m^{2}=100 \mathrm{eV}^{2}\right)$ & 0.136 \\
\hline
\end{tabular}

Table 19: Cuts and relative efficiencies for the detection of $e^{+}-n$ sequences from the $\bar{\nu}_{\mu} \leftrightarrow \bar{\nu}_{e}$ oscillations with the KARMEN experiment.

small oscillation probabilities.

The signature of the detection of $\bar{\nu}_{e}$ is a delayed coincidence (spatially correlated) of energetic positrons from $\bar{\nu}_{e} p \rightarrow e^{+} n$ with energy up to $E_{e^{+}}=E_{\bar{\nu}_{e}}-Q=52.8-1.8=51.0 \mathrm{MeV}$ (Fig. 62). Positrons have to be correlated with $\gamma$ emission from either thermal neutron capture $p n \rightarrow \gamma d$ ( $\gamma$ of $2.2 \mathrm{MeV}$ ) or $n G d \rightarrow \gamma G d$ with on average $3 \gamma\left(\sum E(\gamma)=8 \mathrm{MeV}\right)$ (Fig. 62). Positrons are expected in a time window several $\mu s$ after the beam-on target signal (Fig. 62) with a $2.2 \mu s$ exponential decrease due to $\mu^{+}$decay. Neutrons from $\bar{\nu}_{e} p \rightarrow e^{+} n$ are thermalised and captured within $\simeq 120 \mu s$.
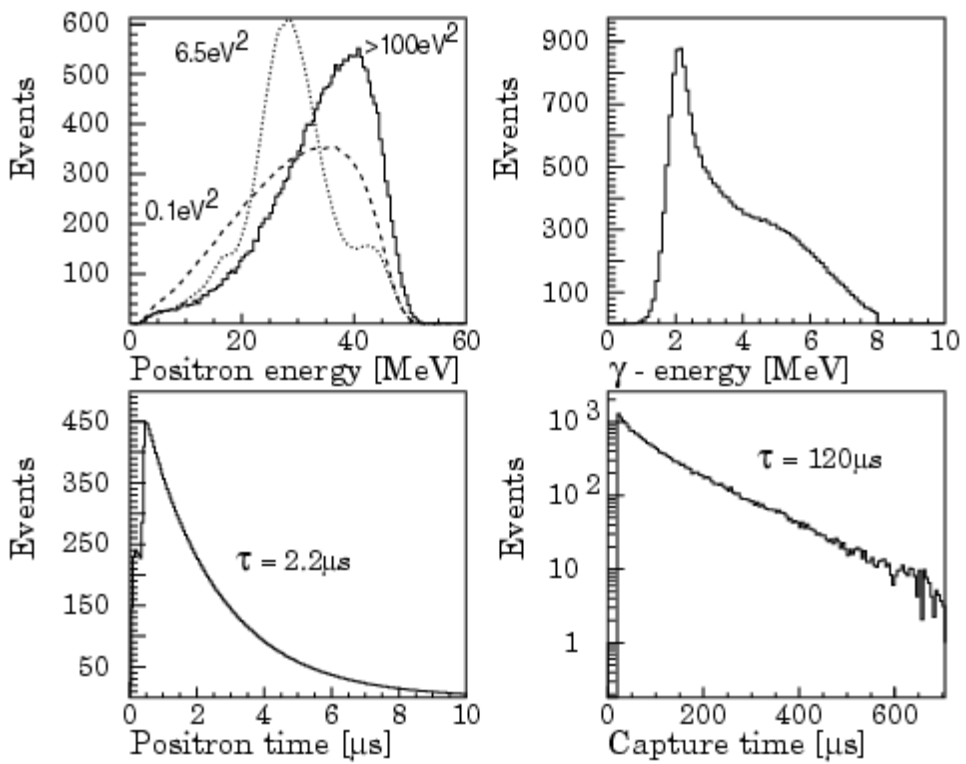

Figure 62: Expected signature of $\bar{\nu}_{\mu} \leftrightarrow \bar{\nu}_{e}$ oscillations in the KARMEN experiment.

The selections applied to detect $e^{+}-n$ sequences originating from $\bar{\nu}_{\mu} \leftrightarrow \bar{\nu}_{e}$ and the relative detection efficiencies are given in Table 19. The neutron efficiency is an average over five years of data taking. It was $14 \%$ at the beginning and $44 \%$ at the end of the running period. The final event sample left after the above cuts consists of 124 events. 


\begin{tabular}{||c|c||}
\hline Background contribution & Events \\
\hline \hline High energy neutrons induced by cosmic rays & $1.9 \pm 0.1$ \\
\hline $\bar{\nu}_{e}$ contamination & $1.1 \pm 0.1$ \\
\hline$\nu$ induced random coincidences & $2.3 \pm 0.3$ \\
\hline$e^{-}-e^{+}$from ${ }^{12} C\left(\nu_{e}, e^{-}\right)^{12} N_{\text {g.s. }}$ & $2.6 \pm 0.3$ \\
\hline \hline Total background & $7.8 \pm 0.5$ \\
\hline
\end{tabular}

Table 20: Expected background for the KARMEN2 experiment.

The main sources of background for the $\bar{\nu}_{\mu} \leftrightarrow \bar{\nu}_{e}$ oscillation search are

- high energy neutron-induced events due to deep-inelastic interactions of cosmic muons in the passive shielding. The expected background from this source amounts to $86.1 \pm 2.3$ events as estimated by measurements between the ISIS pulses;

- $e^{-} e^{+}$sequences from $\nu_{e}^{12} C \rightarrow e^{-12} N_{g s}$ CC interactions;

- events from accidental coincidence of a $\nu$-induced event with a low energy background event;

- events from $e^{+}-n$ sequences induced by the $\bar{\nu}_{e}$ contamination of the beam.

The total expected background amounts to 96.7 events which leads to a beam excess of $27.3 \pm 11.4$. This does not allow to interpret the result in terms of a neutrino oscillation signal.

Thanks to the good energy resolution in the measurement of the $e^{+}$energy and to the dependence of the positron energy spectrum on the oscillations probability a likelihood analysis can be performed to infer the oscillation parameter values. A result compatible with the absence of oscillations is obtained. It corresponds to the $90 \%$ C.L. upper limit on the oscillation probability $P\left(\bar{\nu}_{\mu} \leftrightarrow \bar{\nu}_{e}\right)<4.3 \times 10^{-3}$.

\subsubsection{Oscillation search with the KARMEN2 detector}

The search for $\bar{\nu}_{\mu} \leftrightarrow \bar{\nu}_{e}$ oscillations with the KARMEN2 detector proceeds in a slight different manner from the one developed for KARMEN1. It profits from the upgraded veto system which allows to reduce the influence of the high energy neutron background. Details can be found in [32]. The expected background corresponding to the collected statistics is $7.8 \pm 0.5$ events (Table 20).

8 candidates have been detected within the new analysis. Applying the unified approach [76] to the statistical analysis of small signals the $90 \%$ C.L. upper limit on the oscillation probability $P\left(\bar{\nu}_{\mu} \leftrightarrow \bar{\nu}_{e}\right)<$ $4.2 \times 10^{-3}$ is obtained. This limit is shown graphically in Fig. 58 . It is slightly better than the sensitivity $\left(P\left(\bar{\nu}_{\mu} \leftrightarrow \bar{\nu}_{e}\right)<4.6 \times 10^{-3}\right)$. This fact can be easily explained since, although more sequences than expected are observed, none of these has a prompt event with energy above $36 \mathrm{MeV}$. In this region a background of $0.97 \pm 0.08$ events is expected.

KARMEN will take data until spring 2001. The final sensitivity of the experiment should reach $P\left(\bar{\nu}_{\mu} \leftrightarrow\right.$ $\left.\bar{\nu}_{e}\right)<0.7 \times 10^{-3}$. 


\section{Scenarios for neutrino oscillations}

In the previous Sections we have summarised the available experimental results on neutrino oscillation searches. Three evidences for a positive signal have been reported: the solar neutrino deficit (Section 4), the atmospheric neutrino zenith angle dependence (Section 5) and the LSND excess of $\bar{\nu}_{e}$ and $\nu_{e}$ events (Section 10.1). Also considering the negative results leading to excluded parameter regions, one is faced with the need of a global analysis of all available data.

In the most general case the mixing among three neutrino families can be written as

$$
\left(\begin{array}{l}
\nu_{e} \\
\nu_{\mu} \\
\nu_{\tau}
\end{array}\right)=U_{\alpha i}\left(\begin{array}{l}
\nu_{1} \\
\nu_{2} \\
\nu_{3}
\end{array}\right)
$$

The $3 \times 3$ unitary mixing matrix $U_{\alpha i}$ is described in terms of the Gell-Mann matrices $\lambda_{i}$ by

$$
\begin{aligned}
U_{\alpha i} & =e^{i \psi \lambda_{7}} \Gamma e^{i \phi \lambda_{5}} e^{i \omega \lambda_{2}} \\
& =\left(\begin{array}{ccc}
1 & 0 & 0 \\
0 & c_{\psi} & s_{\psi} \\
0 & -s_{\psi} & c_{\psi}
\end{array}\right)\left(\begin{array}{ccc}
1 & 0 & 0 \\
0 & e^{i \delta} & 0 \\
0 & 0 & e^{-i \delta}
\end{array}\right)\left(\begin{array}{ccc}
c_{\phi} & 0 & s_{\phi} \\
0 & 1 & 0 \\
-s_{\phi} & 0 & c_{\phi}
\end{array}\right)\left(\begin{array}{ccc}
c_{\omega} & s_{\omega} & 0 \\
-s_{\omega} & c_{\omega} & 0 \\
0 & 0 & 1
\end{array}\right) \\
& =\left(\begin{array}{ccc}
c_{\omega} c_{\phi} & s_{\omega} c_{\phi} & s_{\phi} e^{-i \delta} \\
-s_{\omega} c_{\psi}-c_{\omega} s_{\psi} s_{\phi} e^{i \delta} & c_{\omega} c_{\psi}-s_{\omega} s_{\psi} s_{\phi} e^{i \delta} & s_{\phi} c_{\phi} \\
s_{\omega} s_{\psi}-c_{\omega} c_{\psi} s_{\phi} e^{i \delta} & -c_{\omega} s_{\psi}-s_{\omega} c_{\psi} s_{\phi} e^{i \delta} & c_{\psi} c_{\phi}
\end{array}\right)
\end{aligned}
$$

where $c=\cos , s=\sin$ and $\delta$ is the CP-violating phase. Neutrino oscillations can be expressed in terms of six independent variables by combining Eq. (8) and (45): $\Delta m_{21}^{2}=\left|m_{2}^{2}-m_{1}^{2}\right|, \Delta m_{31}^{2}=\left|m_{3}^{2}-m_{1}^{2}\right|, \psi$, $\phi, \omega$ and $\delta$.

Therefore, two independent $\Delta m^{2}$ are available as oscillation parameters. On the other hand, three distinct regions for $\Delta m^{2}$ are allowed by the experimental results: $\Delta m_{\text {solar }}^{2}=10^{-5}$ or $10^{-11} \mathrm{eV}^{2}$, $\Delta m_{\text {atmo }}^{2}=10^{-2}$ to $10^{-3} \mathrm{eV}^{2}$ and $\Delta m_{L S N D}^{2}=0.2$ to $2 \mathrm{eV}^{2}$. Furthermore, one has to accommodate the very large mixing angle obtained in the neutrino sector as compared to the case of the quark sector. In particular, atmospheric neutrino data exclude mixing angles with $\sin ^{2} 2 \theta<0.84$ at the $90 \%$ C.L. [15].

Several phenomenological analyses of the available experimental data have been published [198-204] where the full mixing matrix of a general three-flavour approach is exploited. The problem of how to reconcile all experimental evidences has been attacked by several authors with different methods [205-212]. In the following, we present two of those approaches. The first one adopts the three-flavour scenario with some evidences thrown-out or stretched. The second one assumes the existence of a fourth sterile neutrino leaving room for another $\Delta m^{2}$ value. For an extensive theoretical review of the four-neutrino mixing we refer to [65] and references therein.

\subsection{Three-flavour versus sterile neutrino scheme}

A scheme proposed by B. Kayser [205] consists in neglecting the LSND result as still in need of confirmation. The neutrino mass spectrum shown in Fig. 63 is obtained: $\Delta m_{32}^{2}$ accounts for the atmospheric neutrino results, $\Delta m_{21}^{2}$ incorporates the solar neutrino data and $\nu_{1}, \nu_{2}$ and $\nu_{3}$ are the neutrino mass eigenstates. 


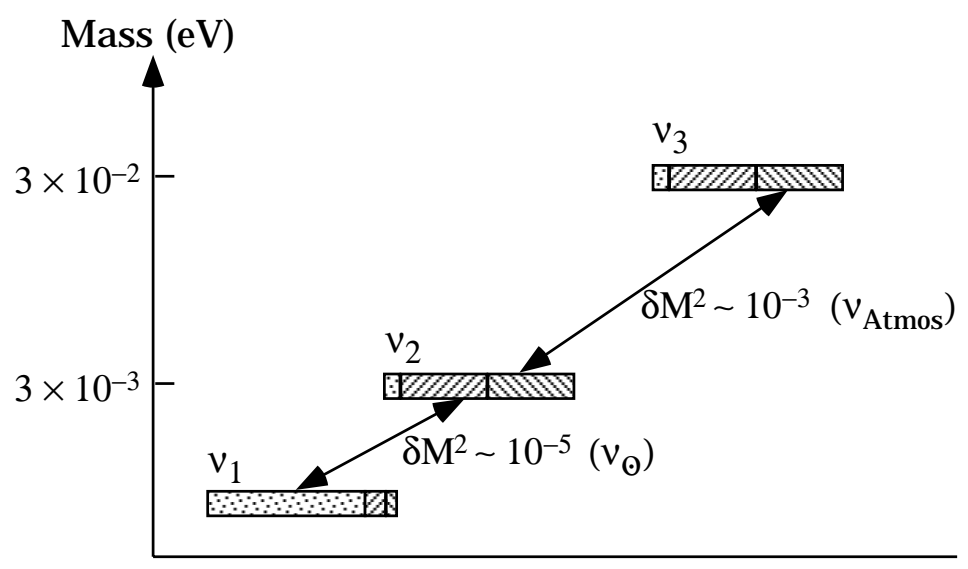

Figure 63: A three-neutrino mass hierarchy which accounts for the oscillation signal from atmospheric and solar neutrinos.

The $\nu_{e}$ content of a given mass eigenstate is dotted, the $\nu_{\mu}$ is indicated by right-leaning hatching and the $\nu_{\tau}$ by left-leaning hatching.

The hierarchy depicted in Fig. 63 is the lightest one which gives the $\Delta m^{2}$ values required by atmospheric and solar neutrino data. Since oscillations determine only mass splittings and not the actual masses one can increase the masses without affecting the oscillations, provided one keeps constant the $\Delta m^{2}$ values. For example, if neutrinos significantly contributed to $\Omega_{M}$ (the total mass density of the Universe expressed as a fraction of the critical density required to stop indefinite expansion) then $m_{1} \simeq m_{2} \simeq m_{3} \simeq(1-2) \mathrm{eV}$ with the $\Delta m^{2}$ values as in Fig. 63 .

Evidence has been accumulating on $\Omega_{M} \simeq 0.1-0.4$ [2]. Large part of the mass of the Universe is dark and can be constituted by two components: the Hot Dark Matter (HDM) and the Cold Dark Matter $(\mathrm{CDM})^{3}$. Neutrinos are natural candidates for the HDM. If $\Omega_{M} \lesssim 0.4$ and the mass density of CDM is at least 0.2 , the mass density of HDM obeys the constraint $\Omega_{H D M} \lesssim 0.2$. On the other hand, the neutrino contribution to the cosmological density can be expressed as $\Omega_{\nu}=\sum_{i=1}^{3} m_{\nu_{i}} /\left(h^{2} 92 \mathrm{eV}\right)$ where $h$ is the Hubble expansion parameter whose value (in units of $100 \mathrm{~km} / \mathrm{Mpc}$ ) ranges from 0.6 to 0.8 [1]. If neutrinos are lighter than $1 \mathrm{keV}$, as it appears to be the case, then $\Omega_{\nu} \leq \Omega_{H D M}$. Therefore, from the previous relations one can deduce that $\sum_{i=1}^{3} m_{\nu_{i}} \lesssim 6-12 \mathrm{eV}$.

If the three-flavour scheme is assumed without neglecting any experimental evidence, one of the following hypotheses must hold

- $\Delta m_{\text {solar }}^{2} \approx \Delta m_{\text {atmo }}^{2}$

- $\Delta m_{L S N D}^{2} \approx \Delta m_{\text {atmo }}^{2}$

- $\Delta m_{\text {atmo }}^{2}$ is a convolution of $\Delta m_{\text {solar }}^{2}$ and $\Delta m_{L S N D}^{2}$.

The first two possibilities are difficult to accommodate given the allowed parameter intervals shown in Fig. 1. The third possibility has been contemplated by several authors [206-212]. The basic idea is that in a

\footnotetext{
${ }^{3} \mathrm{HDM}(\mathrm{CDM})$ consists of particles lighter (much heavier) than $1 \mathrm{keV}$.
} 


\begin{tabular}{||c|c|c|c||}
\hline & $\nu_{\mu} \leftrightarrow \nu_{\tau}$ & $\nu_{\mu} \leftrightarrow \nu_{e}$ & $\nu_{e} \leftrightarrow \nu_{\tau}$ \\
\hline \hline$P\left(\nu_{\alpha} \leftrightarrow \nu_{\beta}\right)$ & 0.3 & 0.03 & 0.10 \\
\hline \hline
\end{tabular}

Table 21: Oscillation probability for the different channels in the scenario discussed in Section 11.1.

three-flavour scheme one expects a complex oscillation phenomenology with transitions between all neutrino pairs. The $\nu_{e}$ solar neutrino flux reduction is assumed to be of a factor two and experiment independent. The last assumption is equivalent to assume the energy independence of the solar neutrino deficit since different experiments probe different regions of the solar neutrino spectrum. This hypothesis is quite reasonable for all but the chlorine experiment (Section 4).

For an example of the above approach, we report the study by G. Barenboim and F. Scheck [210] which accounts for all experimental data by assuming three-flavour mixing with $\Delta m_{32}^{2} \simeq 0.3 \mathrm{eV}^{2}$ and $10^{-4} \lesssim \Delta m_{21}^{2} \lesssim 10^{-3}$. The $\Delta m_{32}^{2}$ value is dictated by the LSND results constrained by the Bugey reactor experiment [213] and by CDHSW (Section 7.2). The lower bound on $\Delta m_{21}^{2}$ is suggested by the SuperKamiokande results. The upper bound is obtained from the CHOOZ experiment: $\Delta m_{21}^{2} \leq 10^{-3} \mathrm{eV}^{2}$. In this approach the Super-Kamiokande results can be interpreted as a combination of $\nu_{\mu} \leftrightarrow \nu_{\tau}$ and $\nu_{\mu} \leftrightarrow \nu_{e}$.

If data are analysed in the framework of the two-flavour mixing, the extracted $\Delta m^{2}$ is a convolution of the two real $\Delta m^{2}$ values. This could also lead to an unphysical best fit for $\sin ^{2} 2 \theta$ (larger than unity) as obtained by the atmospheric neutrino experiments. For this to be an acceptable hypothesis the Super-Kamiokande data must accommodate $\nu_{\mu} \leftrightarrow \nu_{e}$ with small $\Delta m^{2}$ due to the CHOOZ constraint. A consequence of such interpretation would be an excess of $e$-like data in the lowest $\cos \theta_{z}$ (upward going events).

Neglecting the CP-violating phase, according to [210], there are two $U_{\alpha i}$ matrices (Eq. (45)) which satisfy all the evidences and the negative searches for neutrino oscillations

$$
\begin{aligned}
& U_{\alpha i}=\left(\begin{array}{ccc}
0.793 & 0.566 & 0.266 \\
-0.601 & 0.662 & 0.447 \\
0.103 & -0.490 & 0.865
\end{array}\right) \\
& U_{\alpha i}=\left(\begin{array}{ccc}
0.566 & 0.792 & 0.266 \\
-0.783 & 0.432 & 0.447 \\
0.257 & -0.430 & 0.865
\end{array}\right)
\end{aligned}
$$

Assuming the mixing matrices (46) and (47) one can compute the oscillation probability for a given channel. For example, an experiment located $730 \mathrm{~km}$ away from the source using a neutrino beam with mean energy of $10-20 \mathrm{GeV}$ (conditions which apply to the future LBL experiments at NuMI or CNGS) can obtain the results shown in Table 21.

The approach discussed in this Section which discards the energy dependence of the solar results has been questioned by other authors [214] who repeated the same analysis and ... find that the resulting zenith angle distributions of muons are mildly distorted and that the model is disfavoured by the SuperKamiokande atmospheric data at $>99 \%$ C.L. with or without matter effects. We mention that, for the choice of parameters in [209,210], matter effects influence significantly the zenith distributions 
making them flatter than in vacuum. The semi-quantitative calculations in [209,210] showed a more optimistic agreement to the Super-Kamiokande data, in part because matter effects were ignored. In addition, the large value for $U_{\tau 3}^{2}$ chosen in [209,210] does not appear in agreement with the global analysis of laboratory neutrino oscillation searches (including the LSND data) performed in [215].

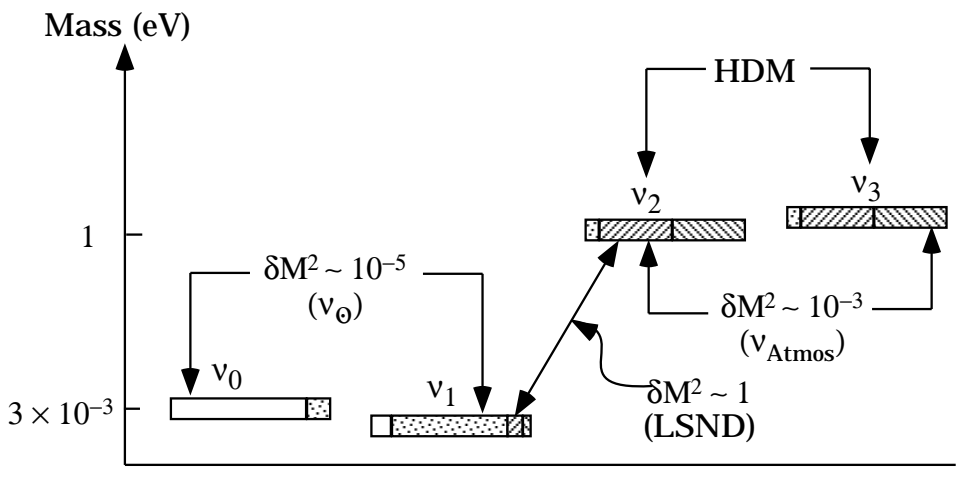

Figure 64: A four-neutrino mass hierarchy which accounts for the oscillation signals from atmospheric, solar and LSND neutrinos.

The second approach in trying to combine all the experimental results is to introduce a (fourth) sterile neutrino $\nu_{s}$. In this case four mass eigenstates are available and the three independent $\Delta m^{2}$ solutions indicated by the experiments can be simultaneously accommodated. A possible neutrino mass spectrum [216] is depicted in Fig. 64 where $\nu_{1}, \nu_{2}, \nu_{3}$ are the usual mass eigenstates and $\nu_{0}$ is the additional one.

In the above scheme there are two pairs of mass eigenstates almost degenerate. The lighter one $\left(\nu_{0}\right.$ and $\nu_{1}$ ) with a splitting of $10^{-5} \mathrm{eV}^{2}$ would explain the solar neutrino deficit interpreted as a $\nu_{e}$ conversion into a $\nu_{s}$. The heavier state $\left(\nu_{2}\right.$ and $\left.\nu_{3}\right)$ has a splitting of about $10^{-3} \mathrm{eV}^{2}$ and accounts for the atmospheric neutrino results. The splitting of $1 \mathrm{eV}^{2}$ between the heavy pair and the light one would explain the LSND result. As a consequence of this approach the average mass of the heavier eigenstate is about $1 \mathrm{eV}$, enough to give a significant contribution to the HDM. We observe that an inverted mass spectrum is also viable since oscillations are driven by mass-squared differences.

Sterile neutrinos appear commonly and naturally in massive neutrino theories. However, they tend to be heavy with masses in the multi $-\mathrm{GeV}$ range or higher. Light sterile neutrinos with masses of $1 \mathrm{eV}$ or less are not usually contemplated.

From the above discussion emerges the need for new data and further studies aimed at a clarification of the experimental and theoretical scenario on neutrino mixing. In the following Sections we describe some of those experiments which have been proposed for future oscillation studies making use of neutrino beams from particle accelerators.

\section{Proposed short baseline experiments}

\subsection{Experiments for $\nu_{\mu} \leftrightarrow \nu_{\tau}$ oscillation searches}

CHORUS and NOMAD have reported no evidence for oscillations at the level of $\sin ^{2} 2 \theta \sim 8 \times 10^{-4}$ for large $\Delta m^{2}$ using only a fraction of their available data $[37,38]$. They will eventually reach a sensitivity 
of $\sin ^{2} 2 \theta \sim 10^{-4}$ for $\Delta m^{2} \geq 100 e V^{2}$. A positive result would allow to infer a $\nu_{\tau}$ with a mass of the order of $10 \mathrm{eV}$. The sensitivity of CHORUS and NOMAD to a lighter $\nu_{\tau}$ in the range of $1-5 \mathrm{eV}$ is $\sin ^{2} 2 \theta \sim 5 \times 10^{-2}$. This sensitivity could be improved by a next-generation short baseline experiment.

Several projects have been proposed in the last years with the aim of exploring small mixing angles in the $\nu_{\mu} \leftrightarrow \nu_{\tau}$ channel, but unfortunately none of them has been approved. Their main common feature is the high sensitivity, achivable by the use of improved and in some cases novel detection methods.

The COSMOS experiment [217] was proposed to run in the future NuMI beam at Fermilab using a detector with a nuclear emulsion target and electronic detectors for the event kinematical analysis. Another experiment employing nuclear emulsions (TENOR) was conceived [218] employing a segmented fully-sensitive large-mass (5 ton) emulsion target in a magnetic field, complemented by high-precision silicon and emulsion trackers as well as by other more conventional electronic detectors. The use of silicon detectors on a large scale, as proposed for the NAUSICAA experiment [219], allows high accuracy in the extrapolation of particle tracks to the emulsion target, needed to yield high signal detection efficiency.

The above arguments contributed to the design and proposal of the TOSCA experiment at the CERN SPS [220]. Its aim is a search for $\nu_{\mu(e)} \leftrightarrow \nu_{\tau}$ oscillations with a sensitivity to $\sin ^{2} 2 \theta_{\mu(e) \tau}$ at least one order of magnitude better than that achievable in CHORUS and NOMAD. The sensitivity to $\Delta m^{2}$ is of the order of $10^{-1} \mathrm{eV}^{2}$ which is outside the region allowed by Super-Kamiokande for $\nu_{\mu} \leftrightarrow \nu_{\tau}$ oscillations. However, some authors [209,210] suggest that $\nu_{\mu, e} \leftrightarrow \nu_{\tau}$ oscillations may occur at $\Delta m^{2} \sim 1 e V^{2}$ and with a small mixing angle $\left(\sin ^{2} 2 \theta_{\mu(e) \tau} \sim 0.06\right)$.

The TOSCA detector consists of units each made of a massive emulsion target followed by high precision silicon micro-strip detectors (Fig. 65). Both are placed in a magnetic field and surrounded by a large acceptance muon detection system. The occurrence of neutrino oscillations is inferred from the detection of $\nu_{\tau}$ CC interactions with a $\tau$ in the final state which identifies the reaction. Similar to CHORUS the $\tau$ identification is carried out by the direct observation of its decay topology in the emulsions, complemented by charge measurement of the decay products and by the decay kinematics for background reduction.

With a target mass of a few tons and exploiting the performance of the SPS (WANF or CNGS) neutrino beam, TOSCA could collect about $5 \times 10^{6}$ neutrino CC interactions in a few years. This large number of events could be entirely scanned without the need of kinematical pre-selection owing to the recent advances in the technique of automatic emulsion scanning (Section 7.5). The high space resolution of the silicon detectors could be used to restrict the emulsion surface area to be scanned, thus resulting in a significant reduction in the analysis time [221].

A large background rejection is achieved by the kinematical analysis of candidate events by measuring the direction of the $\tau^{-}$before its decay. The need for a high efficiency in the measurement of momenta of charged tracks motivates the choice of placing the target in a magnetic field. Past experiments such as WA17 at CERN [222] have already made use of an emulsion target inside an intense magnetic field combined with external tracking devices.

The main source of background in the muonic decay channel is the production of negative charmed particles in anti-neutrino interactions if the primary lepton is not identified. As in CHORUS, the most relevant background to the hadronic decay modes is due to white kinks. The total expected background amounts to about one event for the whole statistics. A sensitivity of $\sin ^{2} 2 \theta_{\mu \tau} \sim 1.8 \times 10^{-5}$ is expected for large $\Delta m^{2}$. The minimum reachable $\Delta m^{2}$ for full mixing is $0.09 \mathrm{eV}^{2}$. 


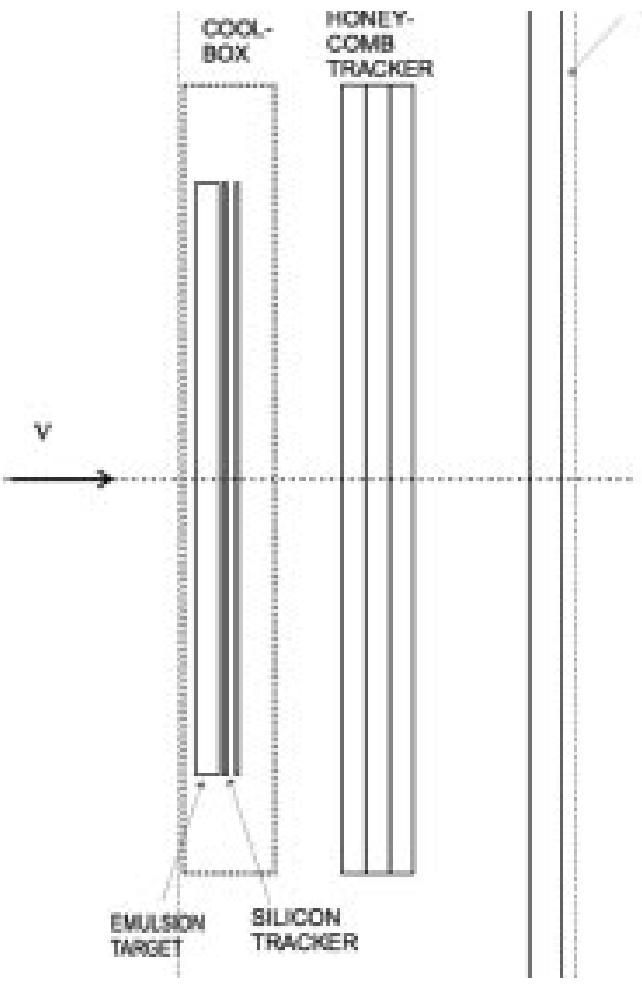

Figure 65: Target unit of the TOSCA detector. The scale is given in millimeters. 


\begin{tabular}{||c|c|c|c||}
\hline & Near & Far & $\Delta_{e}=\left(N_{e} / N_{\mu}\right)^{\text {far }}-C_{e}\left(N_{e} / N_{\mu}\right)^{\text {near }}$ \\
\hline \hline$N_{\mu}$ & $3.4 \times 10^{6}$ & $3.5 \times 10^{5}$ & \\
\hline$N_{e}\left(P\left(\nu_{\mu} \leftrightarrow \nu_{e}\right)=0\right)$ & 12140 & 1200 & $(0.0 \pm 0.8) \times 10^{-3}$ \\
\hline$N_{e}\left(\Delta m^{2}=0.8 \mathrm{eV}^{2}, \sin ^{2} 2 \theta=0.007\right)$ & 12219 & 1656 & $(5.2 \pm 0.5 \pm 0.5) \times 10^{-3}$ \\
\hline
\end{tabular}

Table 22: Number of electron and muon QE events detected by the I216 experiment under different hypotheses. $C_{e}$ is a correction factor which takes into account the difference of the $N_{e} / N_{\mu}$ ratio at the two locations.

\subsection{The I216 experiment in the CERN-PS neutrino beam}

The I216 experiment [34] was proposed to search for the appearance of $\nu_{e}$ in the CERN-PS neutrino beam line (Section 7.2) with the aim of probing the parameter region allowed by the LSND result. The experiment was not approved by CERN, but its main design features are briefly presented in the following.

The $\nu_{\mu} \leftrightarrow \nu_{e}$ oscillation search is performed by comparing the $\nu_{e} / \nu_{\mu}$ event ratio measured in a near detector (one target module $127 \mathrm{~m}$ far from the source) and a far detector (two target modules $885 \mathrm{~m}$ away). The near detector allows the measurement of the $\nu_{e}$ component of the beam, the main source of background, if the oscillation length turns out to be longer than the baseline. The comparison near/far detector gives means to reduce the systematic error down to $3.5 \%$.

The objective of a $\nu_{\mu} \leftrightarrow \nu_{e}$ search in the LSND parameter region defines the detector design: a high mass is needed to explore small $\sin ^{2} 2 \theta$ values as well as good electron identification and high rejection power against $\pi^{0}$ induced events.

The three detector modules have the same structure and are made of a fine-grained tracking calorimeter which constitutes the neutrino target. The basic unit of the detector (plane) is a plastic scintillator layer $\left(3 \times 3 \mathrm{~cm}^{2}\right.$ section, $3.84 \mathrm{~m}$ long) followed by $2 \mathrm{~mm}$ thick iron plates. The near detector consists of 140 planes for a total mass of 104 ton. The far detector includes 640 planes in total and has a mass of 476 ton.

The sampling frequency of the calorimeter is $\sim 0.19 X_{0}$. Such high sampling frequency guarantees the necessary electron identification and $\pi^{0}$ rejection power. The last part of the modules could be instrumented in order to contain electromagnetic showers and extend muon identification and momentum measurement. The design foresees the last 14 scintillator planes of each of the three modules made of iron plates of different thickness. 7 iron plates $2.5 \mathrm{~cm}$ thick are used to sample and absorb electromagnetic showers. These are followed by 7 other plates of $15 \mathrm{~cm}$ thickness which allow to extend the maximum momentum of muons stopping in the near detector to about $3 \mathrm{GeV}$.

Under the assumption of two years' data taking (corresponding to $2.5 \times 10^{20} \mathrm{pot}$ ) the I216 experiment could probe the LSND allowed region with adequate sensitivity (Fig. 66). The results expected for $\Delta m^{2}=$ $0.8 \mathrm{eV}^{2}$ and $\sin ^{2} 2 \theta=0.007$ are shown in Table 22. In that case the experiment would observe an excess of $\sim 450$ events in the far detector and prove the occurrence of oscillations by more than $7 \sigma$.

\subsection{BooNE at the Fermilab Booster}

An experiment with a physics goal analogous to I216 is in preparation at Fermilab. The BooNE experiment (Booster Neutrino Experiment) [33] aims at a search for $\nu_{\mu} \leftrightarrow \nu_{e}$ oscillations both in appearance and disappearance mode in the parameter region indicated by the LSND signal. The first phase of the project 


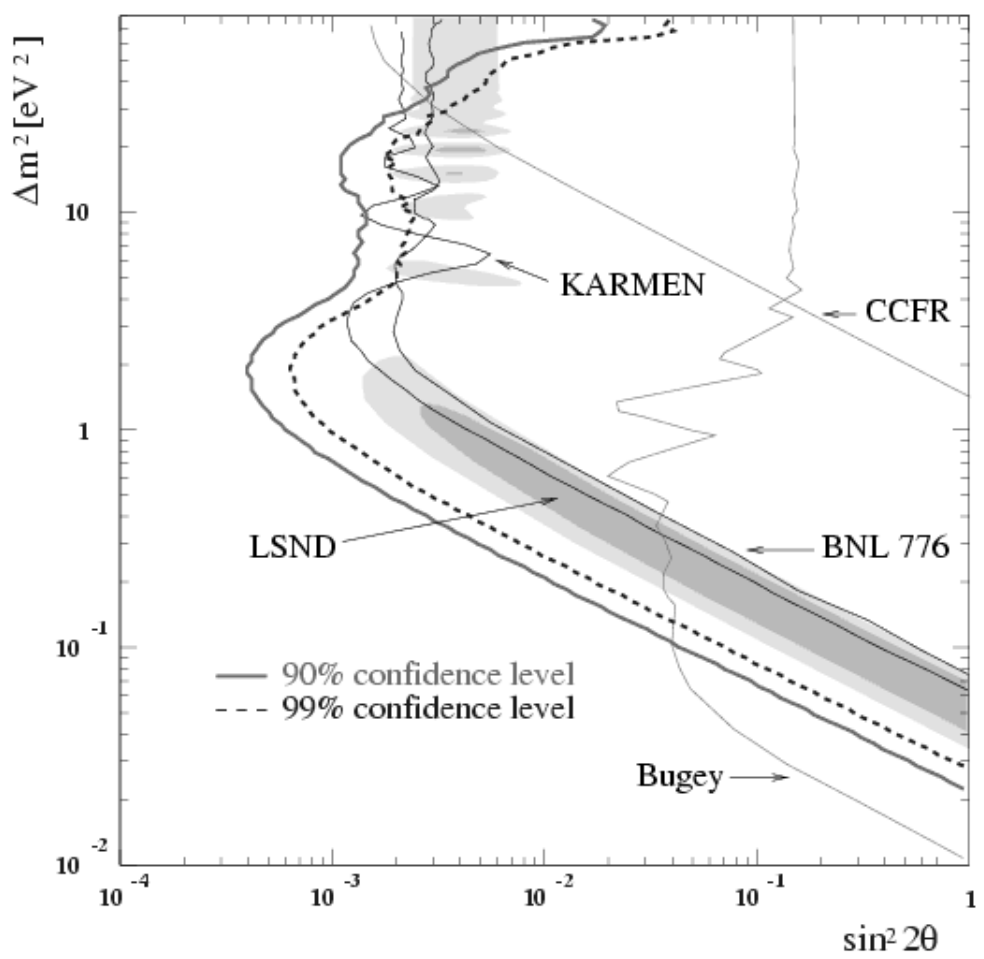

Figure 66: Expected sensitivity of the I216 experiment (90\% C.L.). 
foresees the construction of the MiniBooNE detector placed $500 \mathrm{~m}$ away from the target. Its goal is to collect $\sim 1000$ events per year if the LSND signal is due to $\nu_{\mu} \leftrightarrow \nu_{e}$ oscillations. This will allow to establish the evidence for oscillations at the $8 \sigma$ level. In a later phase of the project a second detector could be installed $1000 \mathrm{~m}$ far from the target with the task of accurately measuring the oscillation parameters.

The BooNE neutrino beam is obtained by sending $8 \mathrm{GeV}$ protons accelerated by the Fermilab Booster onto a titanium target. The Booster delivers $5 \times 10^{12}$ protons at a rate of $15 \mathrm{~Hz}$. Half of them will be used for the future Fermilab programme leaving the rest for BooNE. The focusing system is provided by two horns whose design is similar to the one used at BNL (Section 8). The $50 \mathrm{~m}$ long decay tunnel is filled with helium. The expected spectra $\left(\nu_{\mu}\right.$ and $\left.\nu_{e}\right)$ at the two possible detector locations are shown in Fig. 67.

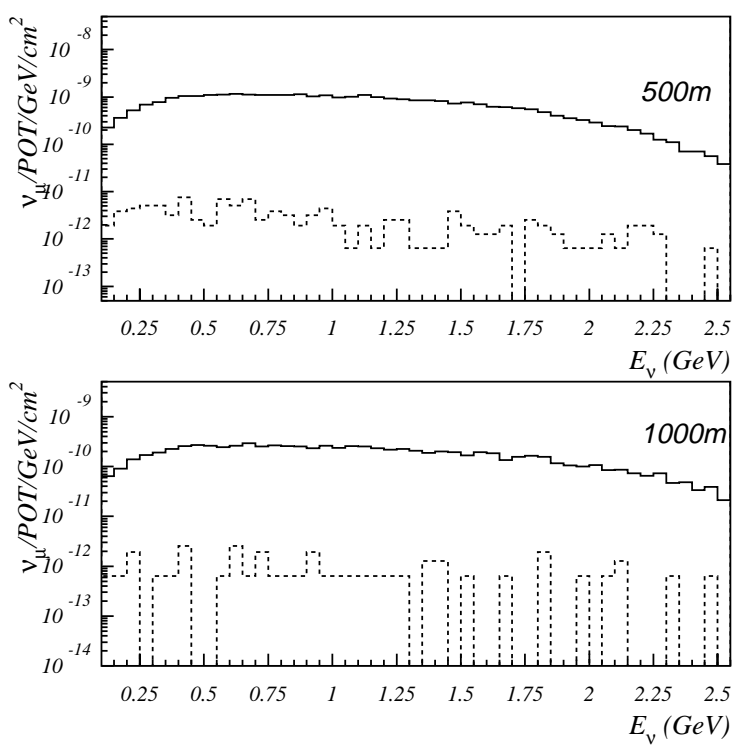

Figure 67: Neutrino spectra for the MiniBooNE experiment at the two different locations.

The MiniBooNE setup is shown in Fig. 68. It consists of a $6 \mathrm{~m}$ radius spherical tank containing 770 ton of pure mineral oil. The volume is split into two parts optically isolated by $1 \mathrm{~cm}$ thick opaque barrier. The inner one is made of a $5.5 \mathrm{~m}$ radius sphere and constitutes the main detector. Electrons generated in $\nu_{e}$ interactions yield $75 \%$ Cerenkov and $25 \%$ scintillation light collected by $12208^{\prime \prime}$ PMTs for a total $10 \%$ surface coverage. The fiducial mass is 445 ton. The outer detector is viewed by 292 PMTs pointing outward and acts as a veto in order to identify uncontained events and reject cosmic-rays.

The signature of $\nu_{\mu} \leftrightarrow \nu_{e}\left(\bar{\nu}_{\mu} \leftrightarrow \bar{\nu}_{e}\right)$ oscillations is the $\nu_{e}\left(\bar{\nu}_{e}\right)$ QE scattering off carbon nuclei $\left(\nu_{e} C \rightarrow\right.$ $e^{-} N$ or $\left.\bar{\nu}_{e} C \rightarrow e^{+} B\right)$. The main sources of background are the intrinsic $\nu_{e}$ contamination of the beam, the mis-identification of $\nu_{\mu} \mathrm{QE}$ scattering and the mis-identification of $\pi^{0} \mathrm{NC}$ production. The analysis is performed by studying the $e^{-}\left(e^{+}\right)$energy distribution which is modified in the presence of neutrino oscillations.

The expected sensitivity of the MiniBooNE experiment is shown in Fig. 69. The estimated numbers of events due to neutrino oscillations and to background processes are shown in Table 23. 


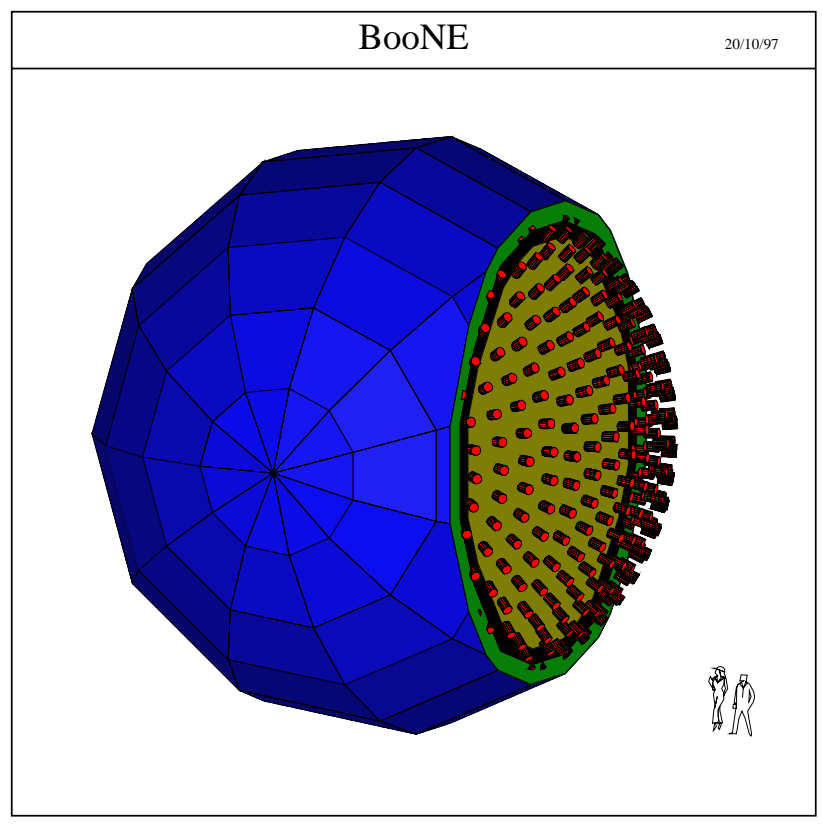

Figure 68: The MiniBooNE detector.

\begin{tabular}{||c|c||}
\hline Process & Number of events \\
\hline \hline Oscillations with $\Delta m^{2}=0.2 \mathrm{eV}^{2}$ and $\sin ^{2} 2 \theta=0.04$ & 675 \\
\hline Oscillations with $\Delta m^{2}=0.4 \mathrm{eV}^{2}$ and $\sin ^{2} 2 \theta=0.02$ & 1200 \\
\hline$\mu^{-}$mis-identification & 600 \\
\hline$\pi^{0}$ mis-identification & 600 \\
\hline \hline Intrinsic $\nu_{e}$ contamination & 1800 \\
\hline Total background & 3000 \\
\hline
\end{tabular}

Table 23: Estimated number of oscillated events and expected background for the MiniBooNE experiment. 


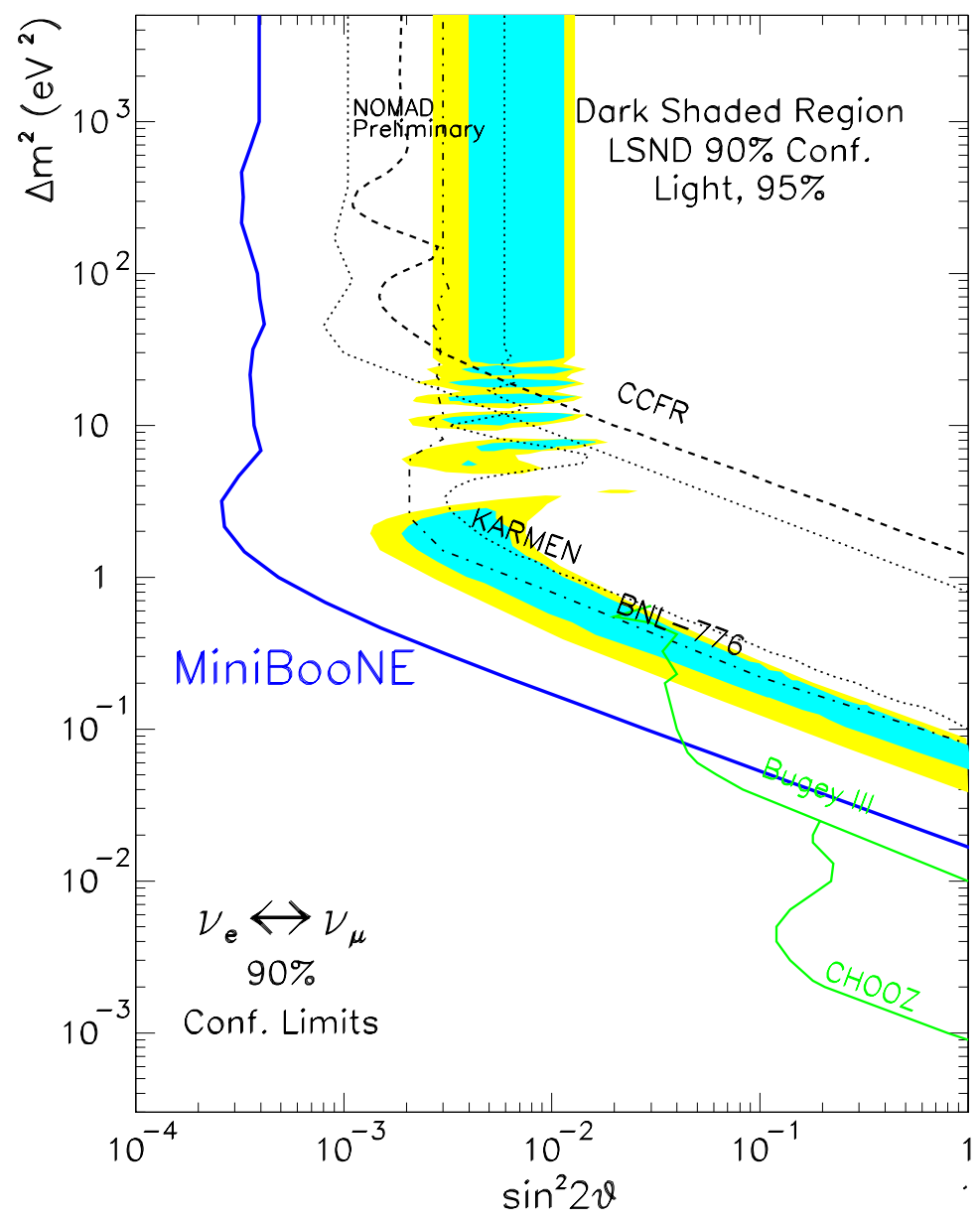

Figure 69: Expected sensitivity of the MiniBooNE experiment. 


\section{Long baseline experiments}

The present experimental evidence from Super-Kamiokande supported by the Kamiokande, Soudan2 and MACRO data together with the CHOOZ results strongly points towards the existence of $\nu_{\mu} \leftrightarrow \nu_{\tau}$ oscillations in the parameter region with $\Delta m^{2} \sim 10^{-2}-10^{-3} \mathrm{eV}^{2}$ and large mixing. The more exotic oscillation of the $\nu_{\mu}$ into a sterile neutrino is also possible although disfavoured by the data (Section 5.2).

In order to further explore the above parameter domain by an independent method, long baseline neutrino-beam experiments are needed. We have seen that for an appearance experiment in the absence of background the sensitivity to $\Delta m^{2}$ improves with the square root of the detector mass and with $1 / E$. There is no dependence on the baseline $L$. This is not true when a background is present. In that case the sensitivity improves with the square root of $L$. This is the main motivation of long baseline experiments: they can benefit from a location at large distance from the neutrino source in order to reduce the number of background events.

Three international projects are in progress or planned aiming at the clarification of the atmospheric neutrino anomaly. We have previously outlined the K2K beam from the KEK Laboratory in Japan, which provides a low-energy neutrino beam pointing towards the Super-Kamiokande detector ( $250 \mathrm{~km}$ away). K2K started operation in 1999. However, it will not allow for a $\nu_{\tau}$ appearance search since the neutrino energy is below the kinematical threshold for $\tau$ production. Another project (NuMI) is approved at Fermilab to exploit the future Main Injector of the upgraded Tevatron accelerator. The neutrino beam will be directed towards the Soudan mine (at $730 \mathrm{~km}$ distance) where the MINOS detector is going to be installed. Both $\nu_{\mu} \leftrightarrow \nu_{\tau}$ indirect appearance and $\nu_{\mu}$ disappearance searches will be performed due to the possibility of varying the neutrino energy. In Europe the CERN CNGS beam will be sent from the SPS accelerator to the Gran Sasso Laboratory (732 $\mathrm{km}$ away) to perform LBL direct $\tau$ appearance experiments with new generation detectors such as ICANOE and OPERA.

In the following, the long baseline experiments for the K2K, NuMI and CNGS facilities are reviewed.

\subsection{The K2K experiment in the KEK-Kamioka beam}

The K2K experiment (KEK to Kamioka) was commissioned in April 1999 [39-42]. It is the first long baseline neutrino oscillation experiment to be operational with an accelerator neutrino beam. A $\nu_{\mu}$ beam (Section 6.5) is sent towards the Super-Kamiokande detector, which is located $250 \mathrm{~km}$ away from KEK. The yield and the characteristics of neutrino events detected with Super-Kamiokande (far detector) are compared with those occurring in the near apparatus installed at the KEK site (near detector). The direct comparison between the response of the two setups largely cancel possible systematic errors in a disappearance search for $\nu_{\mu} \leftrightarrow \nu_{x}$ oscillations. A $\nu_{\mu} \leftrightarrow \nu_{e}$ oscillation appearance search will be performed as well.

The near detector (Fig. 70) is located $300 \mathrm{~m}$ downstream of the target and is made of two distinct types of sub-detectors: a 1 kton water Cerenkov and a fine-grained detector. The first one is a miniature of Super-Kamiokande, the second one is aimed at the determination of the neutrino flux profile and energy distribution at the KEK site.

The fine-grained detector consists of four different elements: a scintillating fiber tracker (SCIFI), trigger counters, lead glass blocks and a muon ranger. The fiber tracker is a 20-layer sandwich of scintillating fiber $(0.7 \mathrm{~mm}$ diameter) sheets and of a water target. The layers are arranged with $9 \mathrm{~cm}$ spacing. The total sensitive transverse area of the tracker is $2.4 \times 2.4 \mathrm{~m}^{2}$. The water target is contained in $1.8 \mathrm{~mm}$ thick 


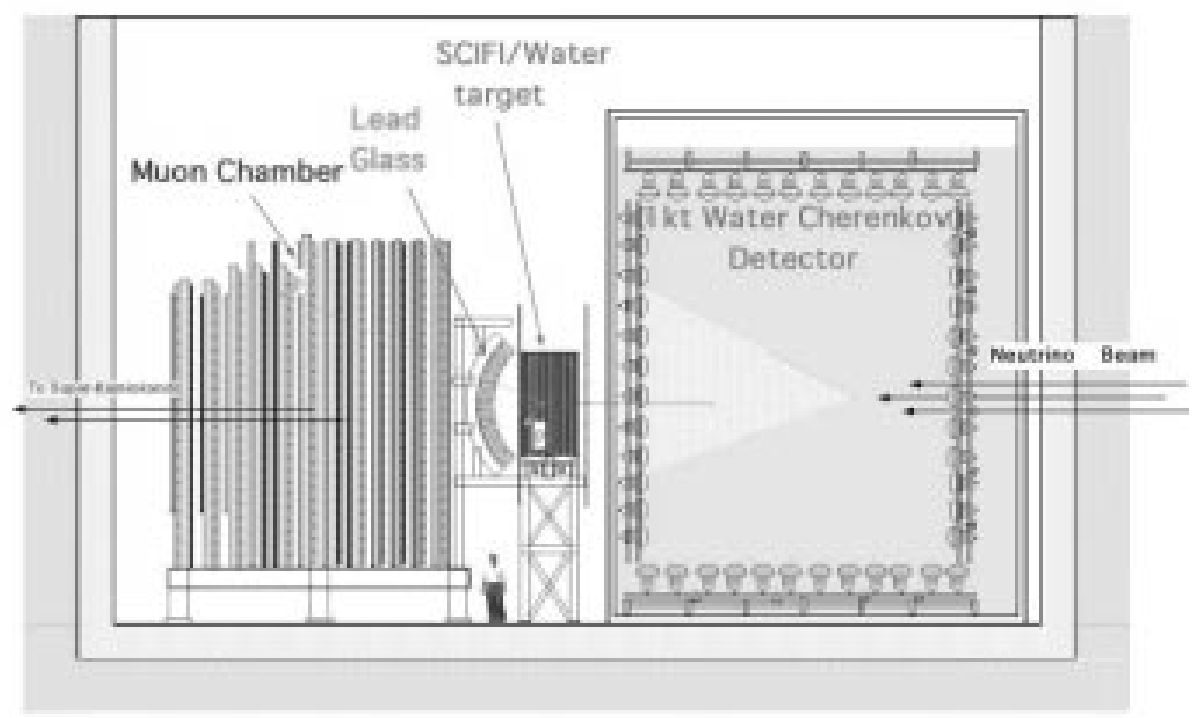

Figure 70: Schematic view of the near detector in the K2K experiment.

aluminium tubes and is placed between the fiber layers.

Scintillating fibers are read out by image intensifier tubes and CCD cameras. The detection efficiency is measured by means of cosmic-ray muons to be better than $99 \%$. The position resolution is $280 \mu m$. The SCIFI/water target is used to reconstruct the tracks of charged particles generated in neutrino interactions and to study the event kinematics. The target is surrounded by about 100 large plastic scintillator plates $\left(4 \times 0.1 \times 0.04 \mathrm{~m}^{3}\right)$. The absolute time measured by these counters (with an accuracy of $0.7 \mathrm{~ns}$ ) is used to reject the cosmic-ray muon background with a reduction rate of $99 \%$.

The task of the lead glass detector is to precisely measure the $\nu_{e}$ contamination of the beam. Each lead glass counter (600 in total) has a $12 \times 12 \mathrm{~cm}^{2}$ geometrical acceptance. The electron/muon separation is performed by using the response of the detector to different particles. The electron energy is measured with a resolution of $8 \% / \sqrt{E_{e}}$ whereas the energy deposited by a passing muon is $\lesssim 1 \mathrm{GeV}$. This allows high energy electrons $(\gtrsim 1.2 \mathrm{GeV})$ to be easily distinguished from muons. The $\nu_{e}$ contamination of the beam can be measured with a $30 \%$ relative error.

The muon ranger consists of about $900 \mathrm{drift}$ chambers and 12 plates of an iron filter $(10-20 \mathrm{~cm}$ thick $)$. Its space resolution is $2.2 \mathrm{~mm}$ and its detection efficiency $99 \%$. The muon momentum is measured by range with a resolution of $\Delta E / E=8-10 \%$. The event display of a simulated of a $\nu_{\mu} \mathrm{QE}$ interaction in the near detector is shown in Fig. 71.

The Super-Kamiokande (far) detector is located in the Kamioka Underground Laboratory. The laboratory has an elevation of $350 \mathrm{~m}$ and it lies under the top of Mt. Ikenoyama, $1350 \mathrm{~m}$ height with a rock overburden of about $1000 \mathrm{~m}$ in the vertical direction.

Super-Kamiokande is a cylindrical large ring-imaging water Cerenkov detector. It detects the Cerenkov light emitted by relativistic charged particles in a massive water volume by means of PMTs arrayed two dimensionally on the detector inner walls. A Cerenkov ring image (which corresponds to a single charged 


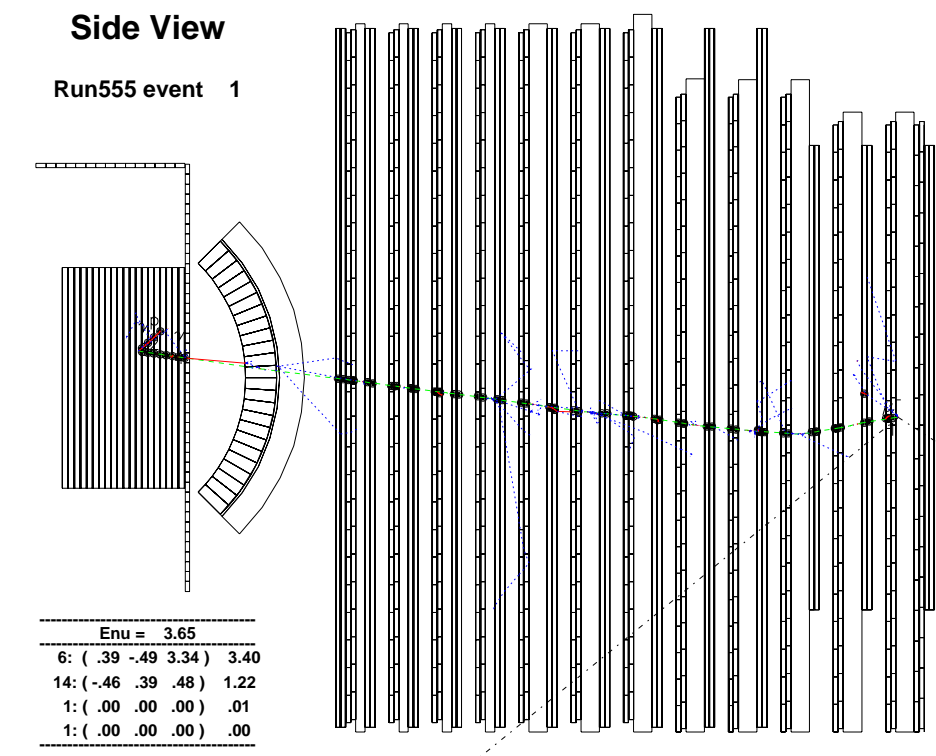

Figure 71: Cross-sectional view of a simulated QE scattering event in the fine-grained near detector of the K2K experiment. 
particle above threshold) is projected onto the detector walls. In the Super-Kamiokande terminology, a single (multi) ring event corresponds to a quasi-elastic (deep-inelastic) interaction. The particle trajectory and the energy are determined by the signal amplitude and timing of each PMT.

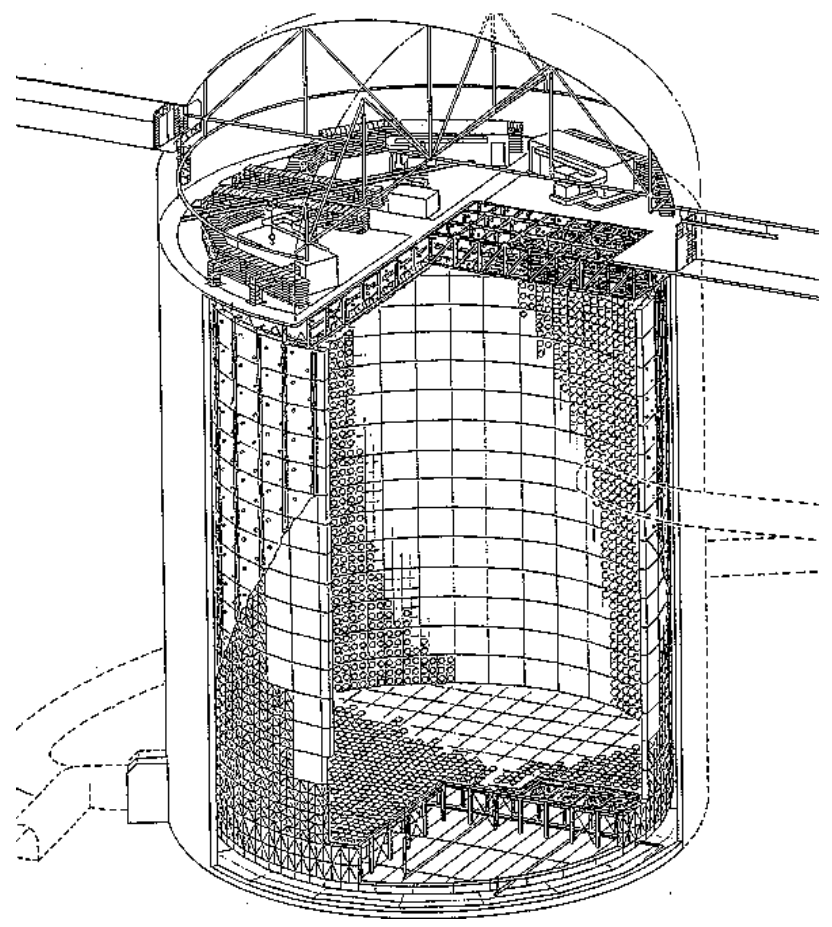

Figure 72: Schematic view of the Super-Kamiokande detector.

Fig. 72 shows a schematic view of the Super-Kamiokande detector. The tank is $42 \mathrm{~m}$ in height, $39 \mathrm{~m}$ in diameter and has a volume of $50000 \mathrm{~m}^{3}$. Super-Kamiokande has two PMT layers: an inner and an outer one. The inner layer constitutes the main detector where the events are observed and is completely surrounded by the outer one. The two layers are optically separated by a pair of opaque sheets as shown in Fig. 73.

In order to obtain the highest photon collection efficiency the inner detector is equipped with 20 " PMTs (Fig. 73). 11146 PMTs are placed uniformly and a $40 \%$ photocathode coverage is achieved, twice that of the Kamiokande experiment. The size of the inner detector inside the PMT surface is $33.8 \mathrm{~m}$ in diameter, $36.2 \mathrm{~m}$ in height and has a volume of $32000 \mathrm{~m}^{3}$.

The outer detector is used as a veto-counter which identifies background particles coming from outside such as cosmic-ray muons. The outer detector also shields the inner one from $\gamma$ rays and neutrons originated from interactions in the cavern rock. Its thickness is $2.05 \mathrm{~m}$ on the side wall and $2.2 \mathrm{~m}$ on the top and bottom walls. The outer detector is equipped with 8 " PMTs (Fig. 73). Each PMT is attached to a squared acrylic plate containing a wavelength shifter to increase light collection efficiency.

The main features of the Super-Kamiokande detector are summarised in Table 24 and compared with those of the former Kamiokande. Typical Super-Kamiokande events, respectively induced by atmospheric $\nu_{\mu}$ and $\nu_{e}$, are shown in Fig. 74 . 


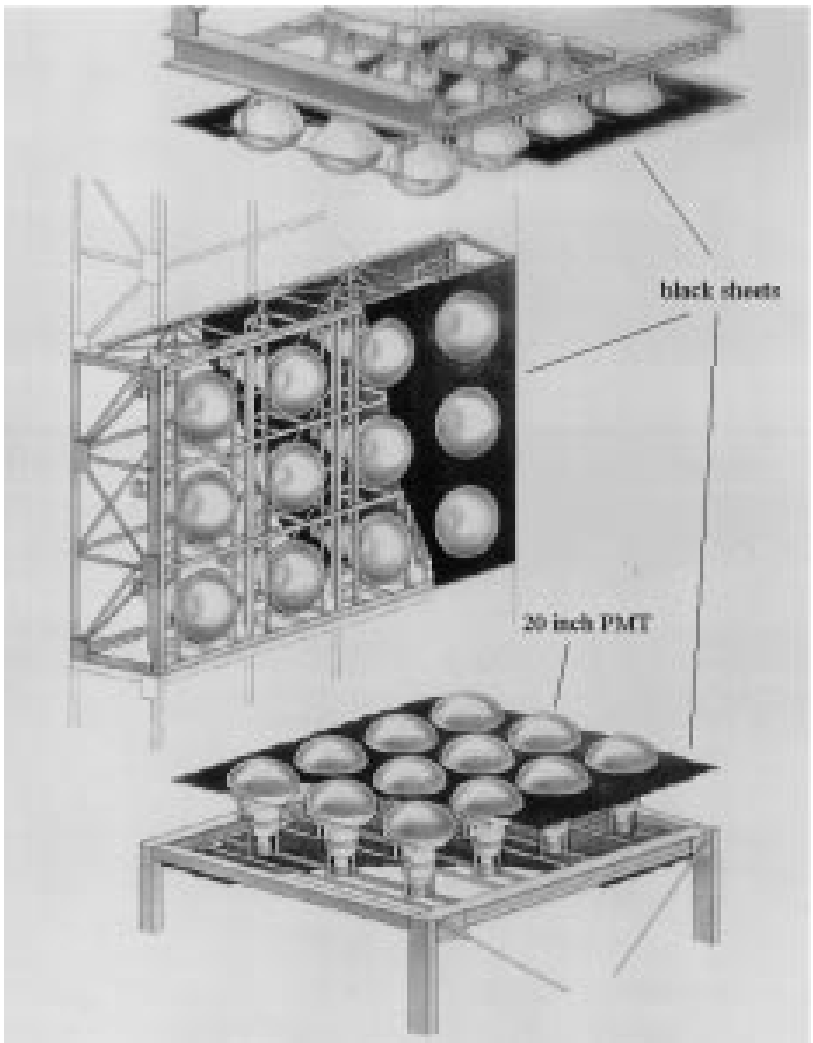

Figure 73: PMT frame structure of the inner and outer detectors of Super-Kamiokande. 


\begin{tabular}{||l|l|l|l||}
\hline Parameters & Super-Kamiokande & Kamiokande & Remarks \\
\hline \hline Total size & $42 m h \times 39 m \phi$ & $16.1 m h \times 15.6 m \phi$ & \\
Total mass & $50000 \mathrm{t}$ & $4500 \mathrm{t}$ & \\
\hline Number of PMTs & $\begin{array}{l}11146\left(20^{\prime \prime}\right) \\
1855\left(8^{\prime \prime}\right)\end{array}$ & $\begin{array}{l}948\left(20^{\prime \prime}\right) \\
123\left(20^{\prime \prime}\right)\end{array}$ & $\begin{array}{l}\text { inner detector } \\
\text { outer detector }\end{array}$ \\
\hline Photosensitive & $40 \%$ & $20 \%$ & \\
coverage & & & at 1 \\
\hline PMT timing & $3 \mathrm{~ns}$ & $4 \mathrm{~ns}$ & photo-electron \\
resolution & & $3.6 \% / \sqrt{E}$ & $e$ of $\mathrm{E}(\mathrm{GeV})$ \\
\hline Energy resolution & $2.6 \% / \sqrt{E}$ & $4 \%$ & $\mu(<1 \mathrm{GeV})$ \\
& $2.5 \%$ & $20 \% / \sqrt{E}$ & $e(<20 \mathrm{MeV})$ \\
\hline Position & $16 \% / \sqrt{E}$ & $110 \mathrm{~cm}$ & $10 \mathrm{MeV}$ \\
resolution & $50 \mathrm{~cm}$ & $15 \mathrm{~cm}$ & $p \rightarrow e^{+} \pi^{0}$ \\
\hline Angular & $\sim 10 \mathrm{~cm}$ & $28^{\circ}$ & $10 \mathrm{MeV}$ \\
resolution & $28^{\circ}$ & $\sim 2^{\circ}$ & Through going $\mu$ \\
\hline$E_{t h}($ trigger $)$ & $\sim 1^{\circ}$ & $5.2 \mathrm{MeV}$ & \\
$E_{t h}($ analysis $)$ & $5 \sim 5 \mathrm{MeV}$ & $7.5 \mathrm{MeV}$ & solar $\nu$ \\
\hline$e / \mu$ separation & $99 \%$ & $98 \%$ & $0.03<p_{e}<1.33 \mathrm{GeV}$ \\
& & & $0.2<p_{\mu}<1.5 \mathrm{GeV}$ \\
\hline \hline
\end{tabular}

Table 24: Experimental features of the Super-Kamiokande and Kamiokande detectors. 


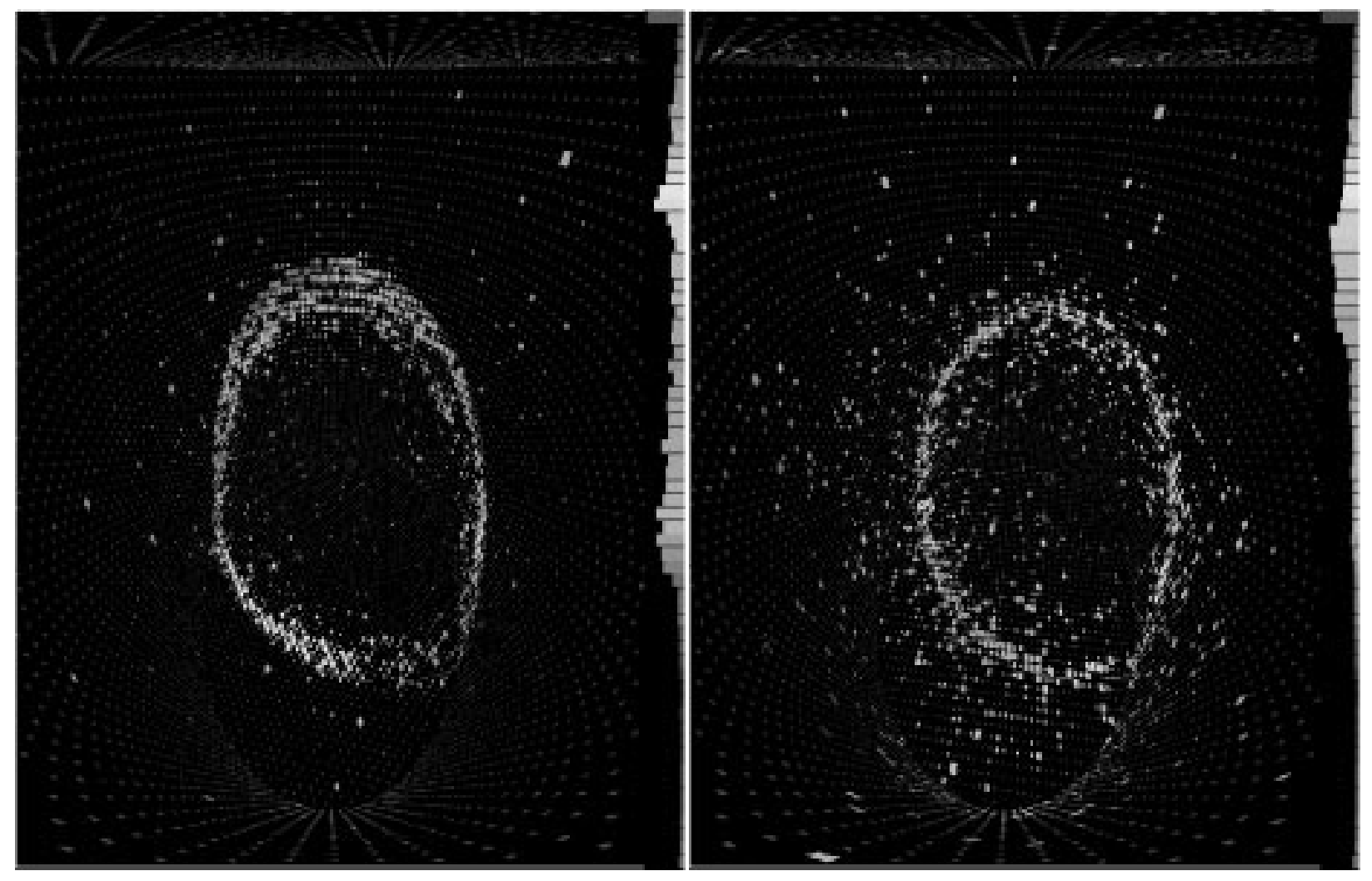

Figure 74: Three-dimensional views of events induced by atmospheric neutrinos in the Super-Kamiokande detector. Left Figure: muon ring induced by a $\nu_{\mu} \mathrm{CC}$ interaction. Right Figure: electron ring produced by a $\nu_{e}$. 

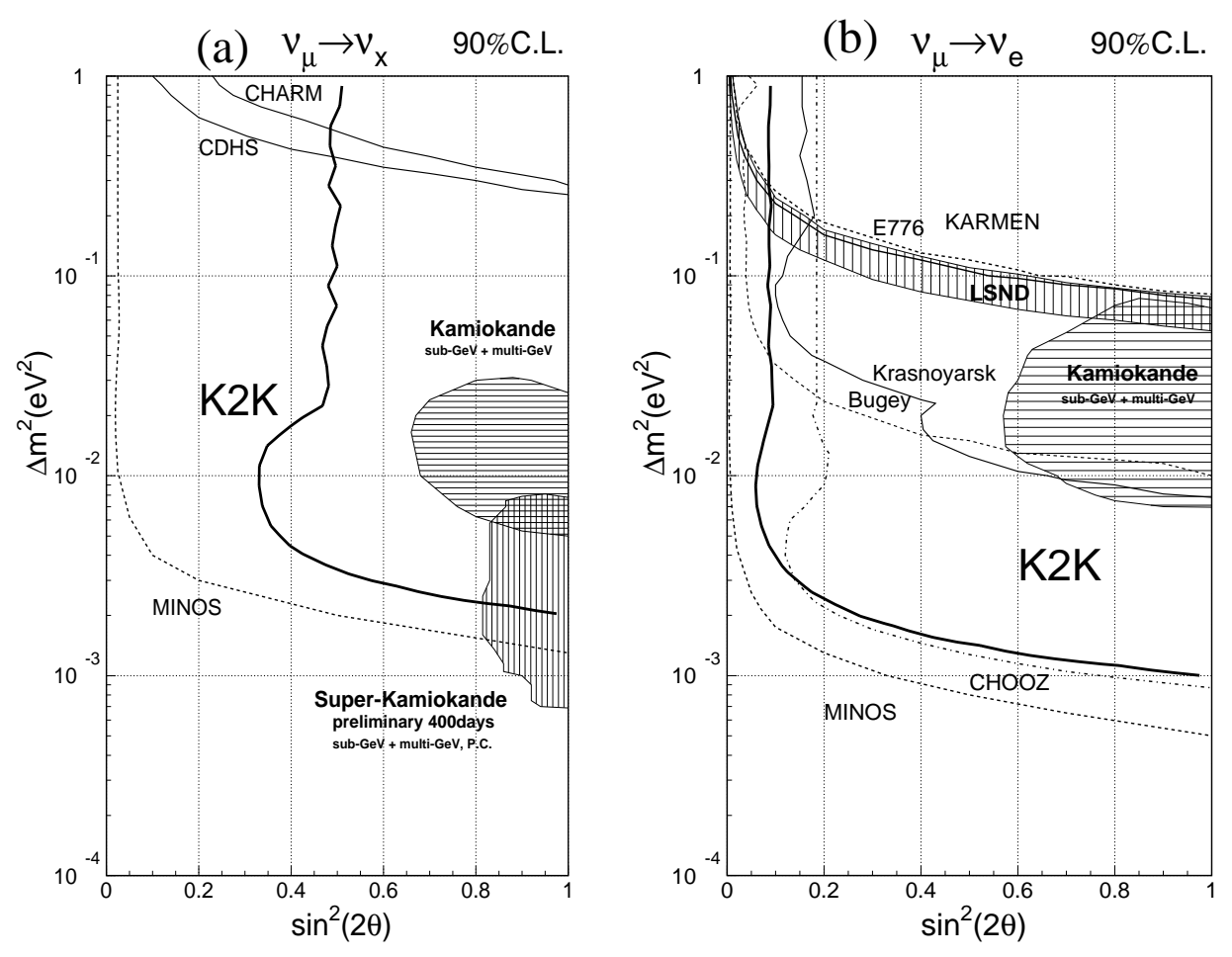

Figure 75: K2K sensitivity at the 90\% C.L. for (a) $\nu_{\mu} \leftrightarrow \nu_{\tau}$ oscillations and (b) $\nu_{\mu} \leftrightarrow \nu_{e}$ oscillations (thick solid lines). The allowed regions by Kamiokande, LSND and Super-Kamiokande and those excluded by other experiments are also plotted. 
$\mathrm{K} 2 \mathrm{~K}$ can perform an appearance search in the $\nu_{\mu} \leftrightarrow \nu_{e}$ channel thanks to the excellent particle identification of the Super-Kamiokande detector. A background to this search is given by $\pi^{0}$ produced in $\nu_{\mu}$ $\mathrm{NC}$ interactions. These events can be recognised due to their low energy (less than $1 \mathrm{GeV}$ ). Assuming oscillation parameters $\left(\Delta m^{2}, \sin ^{2} 2 \theta\right)=\left(10^{-3}, 1\right)$ about 90 electrons with energy above $1.5 \mathrm{GeV}$ are expected, whereas the background from $\pi^{0}$ and from $\nu_{e}$ contamination amounts to $\sim 4$ events. In the case of a negative search the region in the parameter plane excluded at the $90 \%$ is shown in Fig. $75 \mathrm{~b}$. The sensitivity parameter domain is $\Delta m^{2} \gtrsim 1 \times 10^{-3}$ and $\sin ^{2} 2 \theta>0.1$. This region, however, has already been excluded by the CHOOZ experiment [17].

(a)

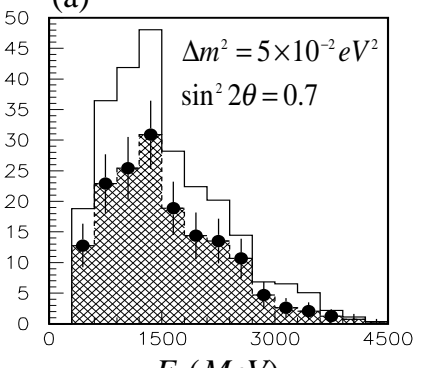

(c) $E_{v}(\mathrm{MeV})$

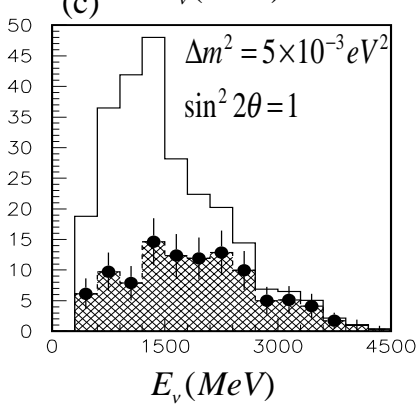

(b)

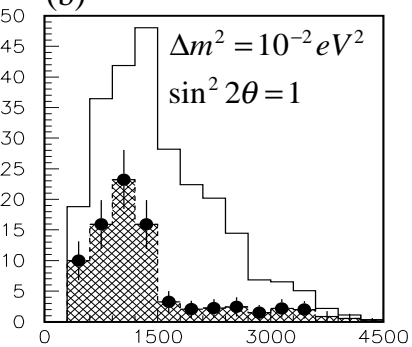

(d) $E_{v}(\mathrm{MeV})$

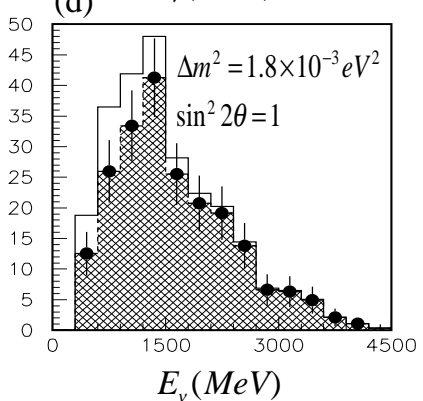

Figure 76: Expected $\nu_{\mu}$ energy spectra with Super-Kamiokande at $10^{20}$ pot for various $\nu_{\mu} \leftrightarrow \nu_{\tau}$ oscillation parameters (a)-(d) (data points with error bars) and in the absence of oscillations (solid histogram).

Due to the low energy of the $\nu_{\mu}$ beam $\left(\left\langle E_{\nu_{\mu}}\right\rangle=1.4 \mathrm{GeV}\right) \mathrm{K} 2 \mathrm{~K}$ cannot perform a $\nu_{\mu} \leftrightarrow \nu_{\tau}$ appearance search. However, $\nu_{\mu} \leftrightarrow \nu_{\tau}$ oscillations can be indirectly detected by measuring the distortion of the neutrino energy spectrum. A reduction of the neutrino flux can be interpreted as an evidence for neutrino oscillations. Expected $\nu_{\mu}$ energy spectra at the Super-Kamiokande site for various $\nu_{\mu} \leftrightarrow \nu_{\tau}$ oscillation parameters are shown in Fig. 76.

The neutrino energy spectrum is derived by using $\nu_{\mu} \mathrm{QE}$ interactions. These events are particularly interesting since they can be recognised as single-rings and the neutrino energy can be calculated from the momentum and the scattering angle of the muon track.

The parameter region which could be excluded by K2K at the $90 \%$ C.L. in the absence of oscillations is shown in Fig. 75a. The experiment is, therefore, sensitive to $\Delta m^{2}>3 \times 10^{-3}$ and $\sin ^{2} 2 \theta>0.4$.

Presently, after a few months of operation, the K2K experiment has already collected events both in the near and in the far detectors. Three neutrino interactions have been observed in the fiducial volume of the Super-Kamiokande detector with $12.3_{-1.9}^{+1.7}$ events expected [223]. An oscillation analysis focusing on the absolute number of events, on the distortion of the neutrino energy spectrum and on the $\nu_{e} / \nu_{\mu}$ ratio is in 
progress. In Fig. 77 the display of the very first event in the Super-Kamiokande detector is shown. It is the first neutrino interaction obtained with an accelerator LBL beam.

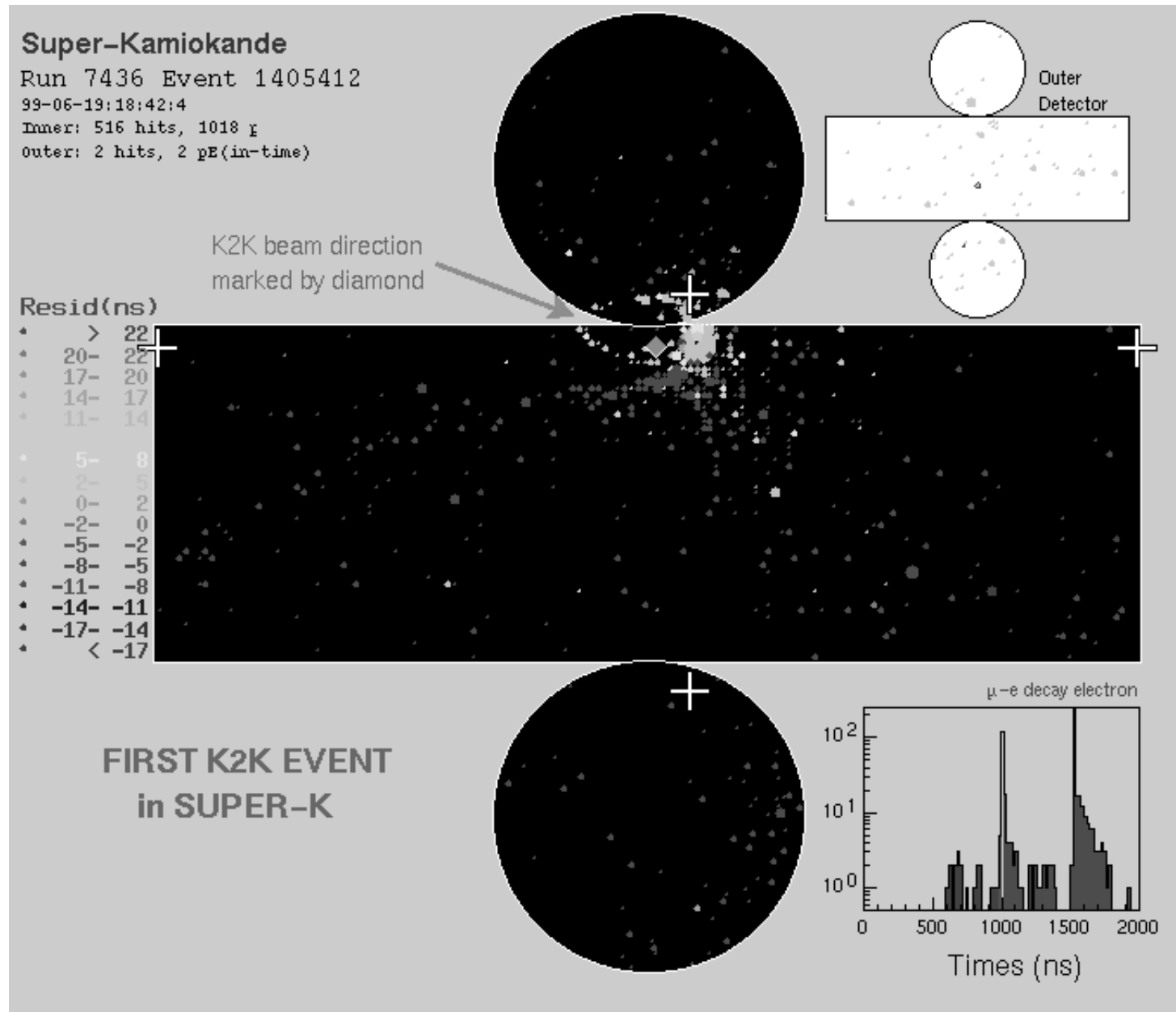

Figure 77: Event display of the first neutrino interaction detected by the K2K experiment with the SuperKamiokande detector.

\subsection{The MINOS experiment in the NuMi beam}

A neutrino programme aimed at the study of neutrino oscillations was proposed since 1989 at Fermilab. The idea is to use the high intensity proton beam of the future Tevatron Main Injector to provide an intense source of neutrinos. The features of the neutrino beam have already been presented in Section 6.4. Here we focus on the MINOS experiment [43] (Main Injector Neutrino Oscillation Search) which has been proposed to search for neutrino oscillations in a long baseline configuration.

MINOS and the NuMI beam have been optimised for a disappearance oscillation search in the parameter region indicated by Super-Kamiokande. Motivated by the requirements of a disappearance experiment the MINOS detector is made up of a near detector located at the Fermilab site (290 $\mathrm{m}$ from the neutrino source) and of a far detector in the Soudan mine in Minnesota $730 \mathrm{~km}$ away. In order to reduce systematic errors 


\section{Schematic of the Near Detector}

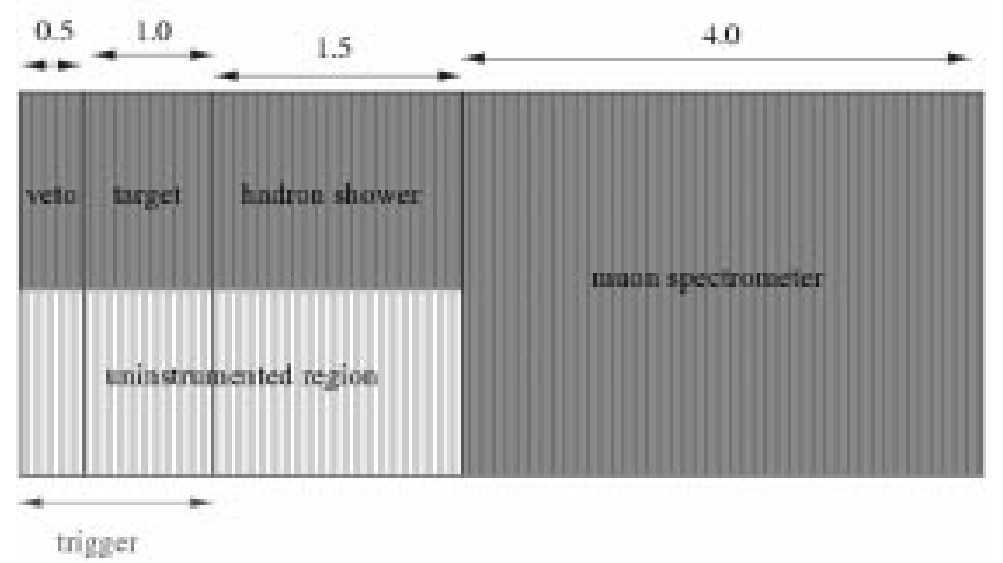

Figure 78: Schematic structure of the MINOS near detector.

\begin{tabular}{||c|c||}
\hline Parameter & Value \\
\hline \hline Energy scale calibration & $5 \%$ absolute, $2 \%$ near-far \\
\hline EM energy resolution & $23 \% / \sqrt{E(G e V)}(<5 \%$ constant term $)$ \\
\hline Hadron energy resolution & $60 \% / \sqrt{E(G e V)}(<7 \%$ constant term) \\
\hline Muon momentum resolution & $<12 \%$ (from curvature or range) \\
\hline Neutral (NC) charged $(\mathrm{CC})$ current event separation & Efficiency $>90 \%(99.5 \%$ with corrections $)$ \\
\hline Electron $/ \pi$ separation & Hadron rejection $\sim 10^{3}$ for $\varepsilon_{e} \sim 20 \%$ \\
\hline \hline
\end{tabular}

Table 25: MINOS experimental parameters.

due to the knowledge of the beam the near setup has a design similar to the far. Both detectors consist of an iron-scintillator calorimeter and have the same absorber thickness, active elements, granularity, magnetic field and geometrical shape.

The mass of the near detector is $\sim 1$ ton. It consists of four parts, as shown in Fig. 78: a veto system made of $50 \mathrm{~cm}$ thick steel allows to reject events induced by neutrons; a neutrino target consisting of a $1 \mathrm{~m}$ thick iron block; a hadron catcher which contains showers induced by the neutrino interactions occurring in the target; a muon spectrometer made of iron plates $(2.54 \mathrm{~cm}$ thick) interleaved with scintillator strips $1 \mathrm{~cm}$ thick, $4.1 \mathrm{~cm}$ wide and $8 \mathrm{~m}$ long which allows to range out muons and/or measure their momentum by curvature.

The far detector is a 5400 ton magnetised iron calorimeter with scintillator strips as active elements. The basic module is a $8 \mathrm{~m}$ diameter, $2.54 \mathrm{~cm}$ thick steel plane followed by a $1 \mathrm{~cm}$ thick scintillator plane. Each scintillator plane is made up of 192 strips, $4 \mathrm{~cm}$ wide and up to $8 \mathrm{~m}$ long, depending on the position in the plane. The orientation of the strips is alternated by $\pm 90^{\circ}$ in successive planes.

A MINOS supermodule is made up of 242 such modules. Two supermodules compose the baseline layout of the MINOS far detector. The average magnetic field in the iron is $1.5 \mathrm{~T}$. A schematic view of the far detector is shown in Fig. 79. The main features of MINOS are presented in Table 25. 


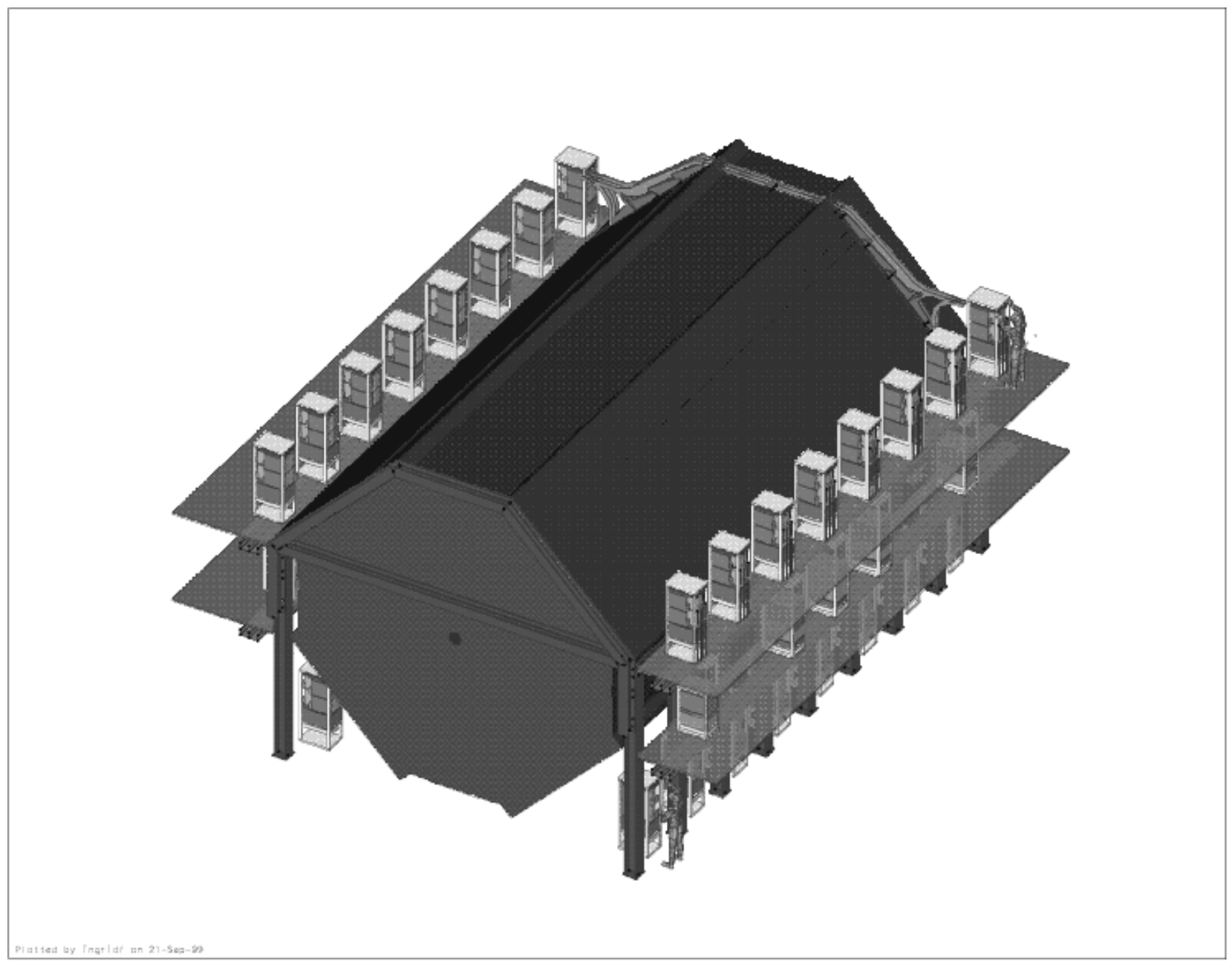

Figure 79: Schematic view of a supermodule of the MINOS far detector. 
The excellent NC/CC event separation together with the good energy resolution of the detector allows three different statistical methods to be exploited in the detection of neutrino oscillations: the near/far test (Section 3.1.1), the NC/CC ratio measurement (Section 3.2.1) and the CC event energy measurement (Section 3.1.2).

As discussed in Section 6.4 three different beams have been considered for the experimental programme. The expected results with the high (PH2 high) and low (PH2 low) energy beams are shown in Fig. 80 and Fig. 81, respectively. Fig. 82 shows the precision achievable by MINOS in the determination of the oscillation parameters with a two years' running. The experiment is presently in its construction phase. It should start data taking in the year 2003 .
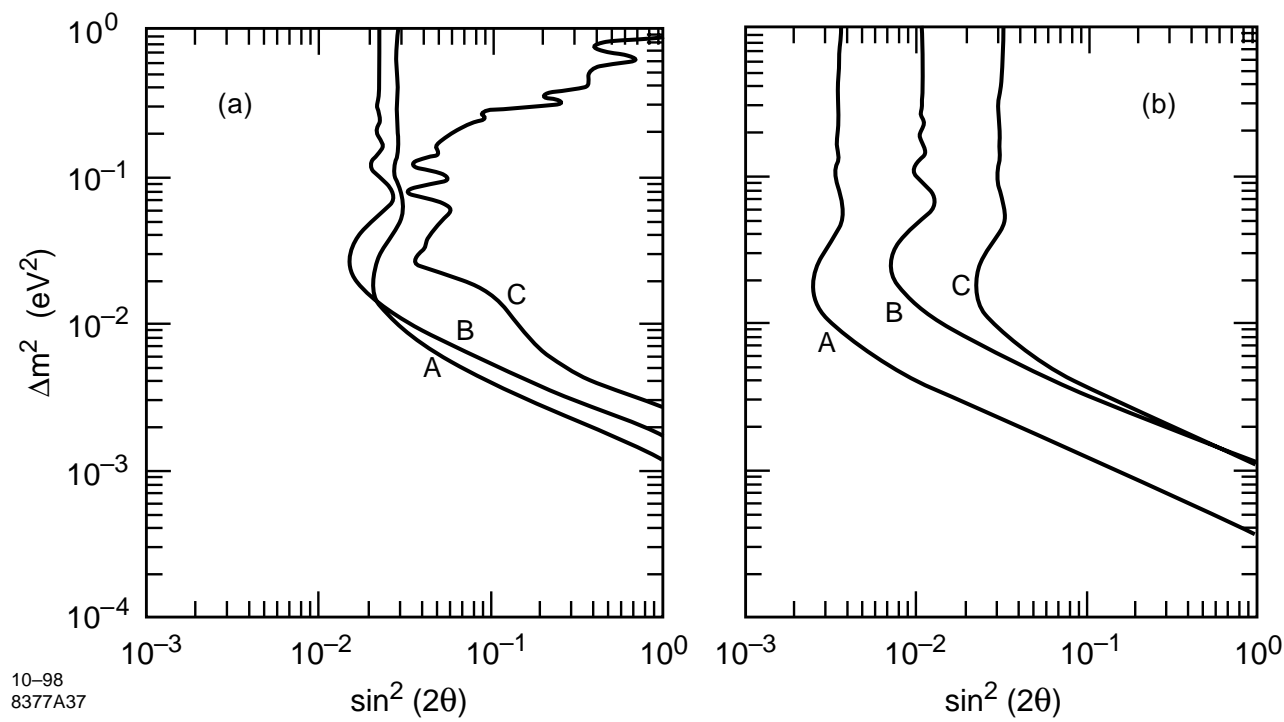

Figure 80: 90\% C.L. limits on neutrino oscillation parameters established by MINOS with 10 kton - year in the absence of oscillations with the PH2 (high) beam. The A, B and C curves show limits obtained from different oscillation tests. (a) $\nu_{\mu} \leftrightarrow \nu_{\tau}$ limits: A refers to the near-far event rate comparison, B to the $\mathrm{NC} / \mathrm{CC}$ ratio test and $\mathrm{C}$ to the CC-event energy test. (b) $\nu_{\mu} \leftrightarrow \nu_{e}$ limits: A stands for the electron appearance test, $\mathrm{B}$ for the $\mathrm{NC} / \mathrm{CC}$ ratio and $\mathrm{C}$ for the near-far event rate comparison.

\subsection{The ICANOE experiment in the CNGS beam}

The ICANOE detector [51,224] results from the common effort of the ICARUS [44] and NOE [45] Collaborations towards the design of a general-purpose underground detector to be installed in the Gran Sasso Laboratory. The underlying idea is to combine the ICARUS liquid argon imaging technique with the NOE fine-grained calorimeter suitably upgraded with magnetic analysis of muons.

The basic element of the ICANOE detector is a supermodule (Fig. 83). It consists of a liquid target of 1.4 kton active mass with about $11 \times 11 \times 18 \mathrm{~m}^{3}$ external dimensions and of a magnetised calorimeter 0.8 kton in weight with dimensions of $\sim 9 \times 9 \times 2.5 \mathrm{~m}^{3}$. The baseline configuration of the detector foresees 4 supermodules for a total 5.6 (liquid) +3.2 (solid) $=8.8$ kton instrumented mass.

The main goal of ICANOE is the study of neutrino masses and mixing by exploiting the CNGS neutrino beam as well as atmospheric and solar neutrinos. The regions in the $\Delta m^{2}-\sin ^{2} 2 \theta$ plane explored by 


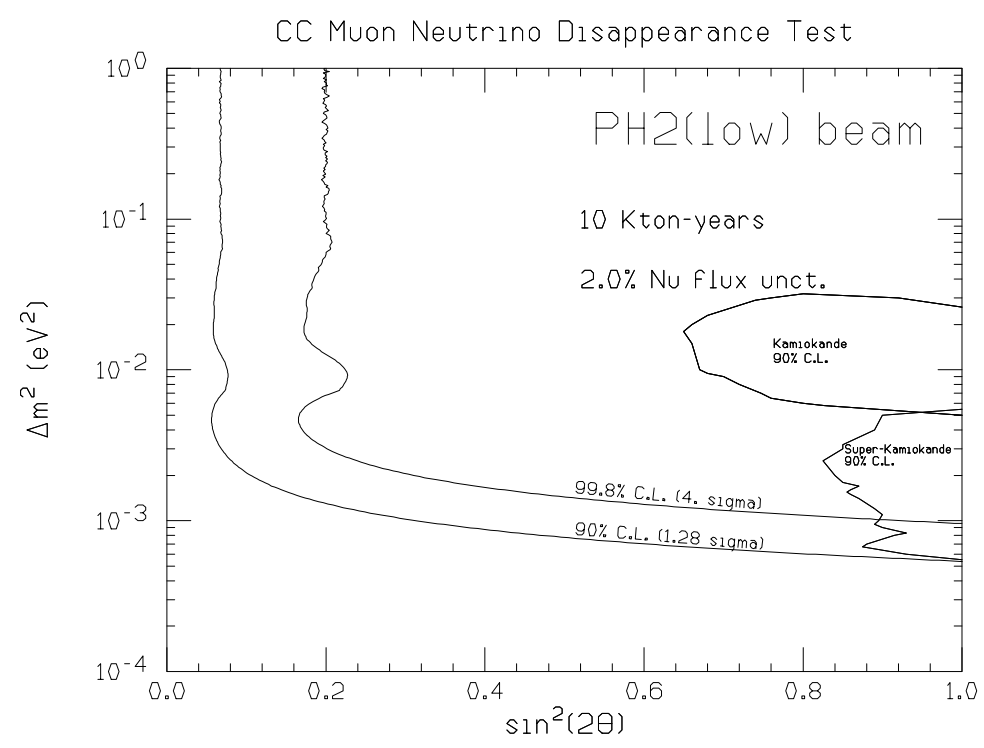

Figure 81: MINOS sensitivity for the $\nu_{\mu}$ disappearance test (90\% C.L. and $4 \sigma$ contour). A 10 kton - year exposure in the low energy PH2 (low) neutrino beam is assumed.

ICANOE are shown in Fig. 84. Here below we briefly discuss the main features of the experiment components, i.e. the ICARUS and NOE detectors, as derived from test results and simulations. The expected ICANOE performance in the CNGS neutrino beam is then described.

\subsubsection{The ICARUS detector}

The aim of the ICARUS Collaboration [44,225] is the construction of a liquid argon time projection chamber (TPC) detector with a space resolution comparable to that of bubble chambers. The ICARUS physics programme includes decay-mode independent proton decay search, model-independent solar neutrino flux measurements as well as search for oscillations with atmospheric and accelerator neutrino beams.

The working principle of the ICARUS detector is simple. A large liquid argon volume is contained in a cryostat equipped with two wire readout planes (one at each end of the volume) and a high voltage plane at the center giving a long drift length (Fig. 85). Ionisation electrons produced by charged particles crossing the liquid argon are collected at the readout planes. The arrival time of the different electrons allows the reconstruction of the particle track trajectory by the time projection technique. Each readout plane has three coordinates at $60^{\circ}$ from each other and wire pitch of $3 \mathrm{~mm}$. This corresponds to a bubble diameter of about $3 \mathrm{~mm}$. This was the bubble size of the Gargamelle detector at CERN [124].

The detector is a homogeneous tracking device capable of $d E / d x$ measurement. Its high $d E / d x$ resolution allows good momentum measurement and particle identification of soft particles. Electromagnetic and hadronic showers are, in fact, fully sampled. This gives the energy resolution for electromagnetic showers of $\sigma(E) / E=3 \% / \sqrt{E(G e V)} \oplus 1 \%$ and for hadronic contained showers of $\sigma(E) / E=16 \% / \sqrt{E(G e V)} \oplus 1 \%$. ICARUS features excellent electron identification and $e / \pi^{0}$ separation.

A small prototype (50 liters) of the ICARUS detector has been successfully exposed to the CERN WANF neutrino beam [226]. A larger module of 600 ton is under construction and expected to be soon 

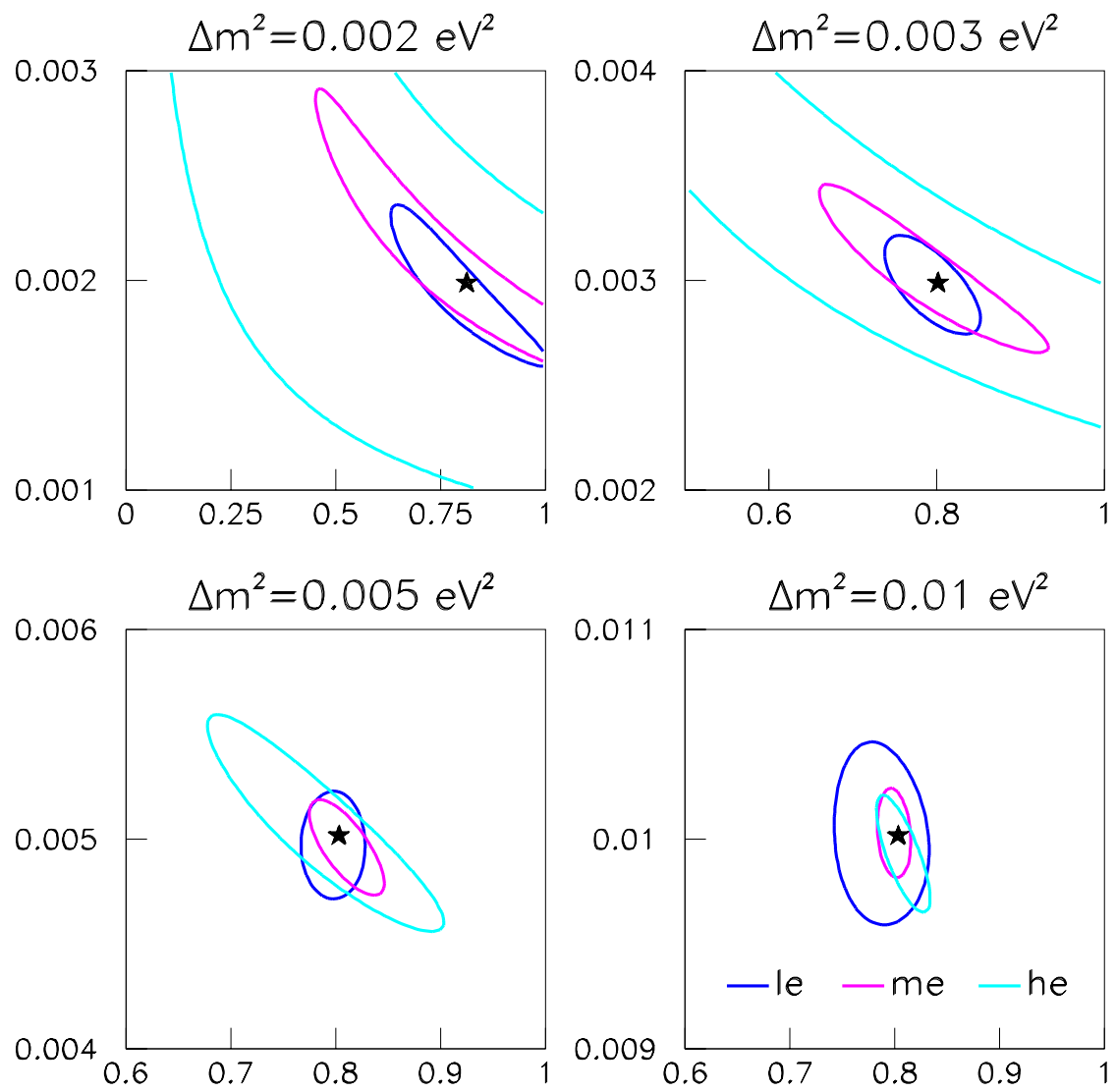

Figure 82: The $68 \%$ C.L. contours relative to an oscillation signal with various $\Delta m^{2}$ values and $\sin ^{2} 2 \theta=0.8$ as obtained by MINOS in two years' running. Different contours are obtained by using different beams: low, medium and high energy. 


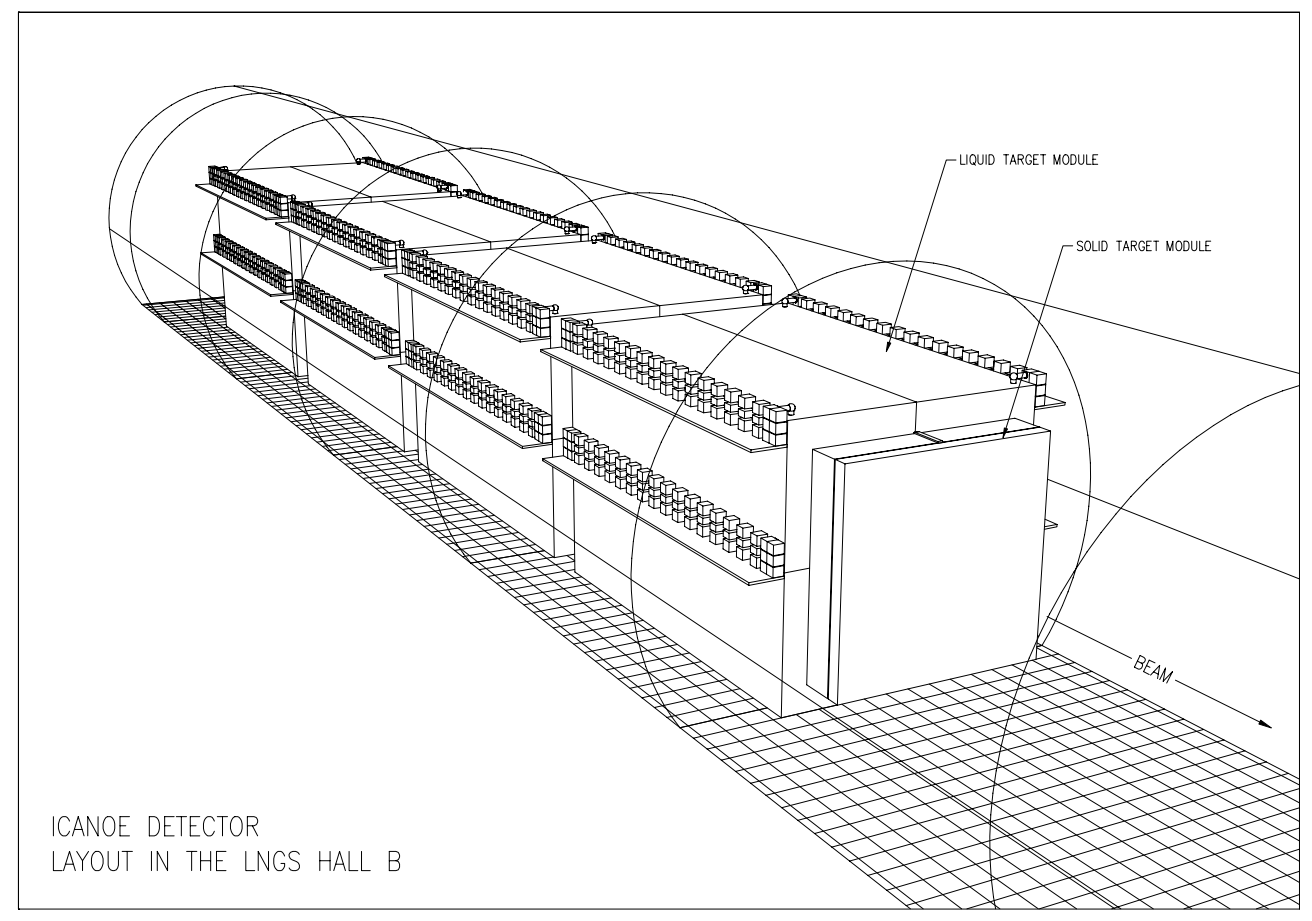

Figure 83: Schematic view of the ICANOE detector. 


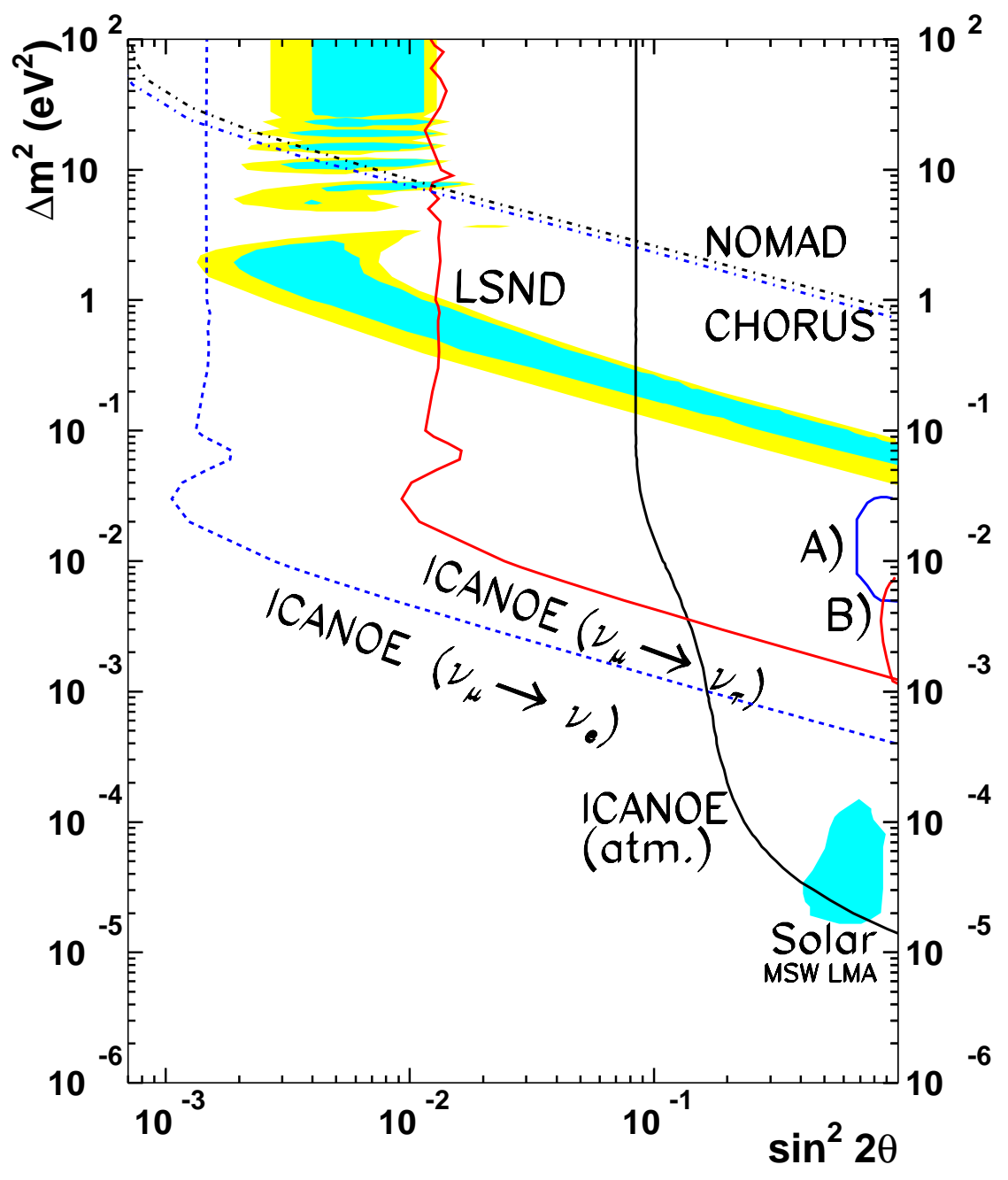

Figure 84: ICANOE sensitivities at the 90\% C.L. are indicated by three curves: the observation of atmospheric neutrinos, the $\nu_{\tau}$ appearance search at the CNGS and the $\nu_{e}$ appearance search at the CNGS. 


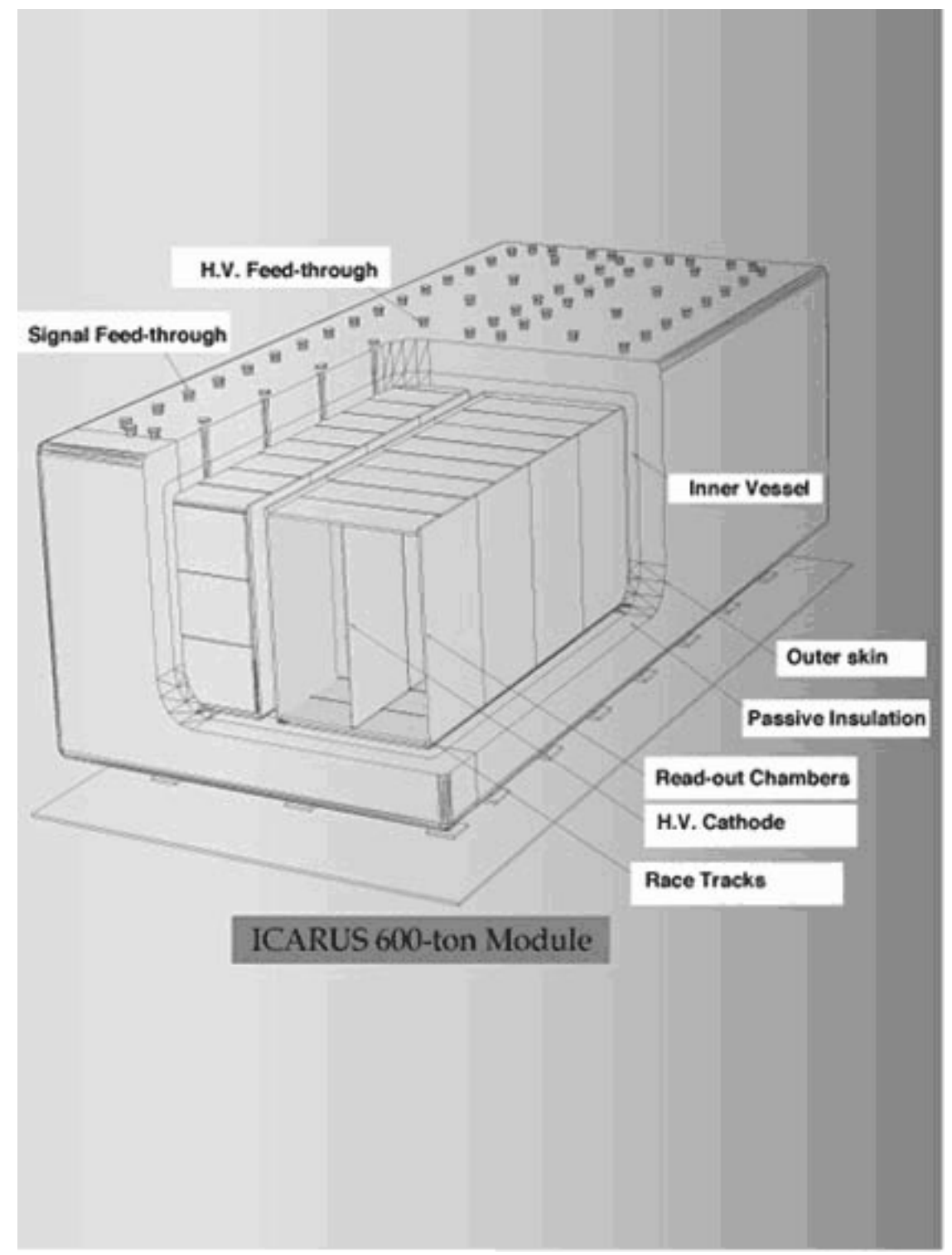

Figure 85: Schematic view of the 600 ton ICARUS detector. 


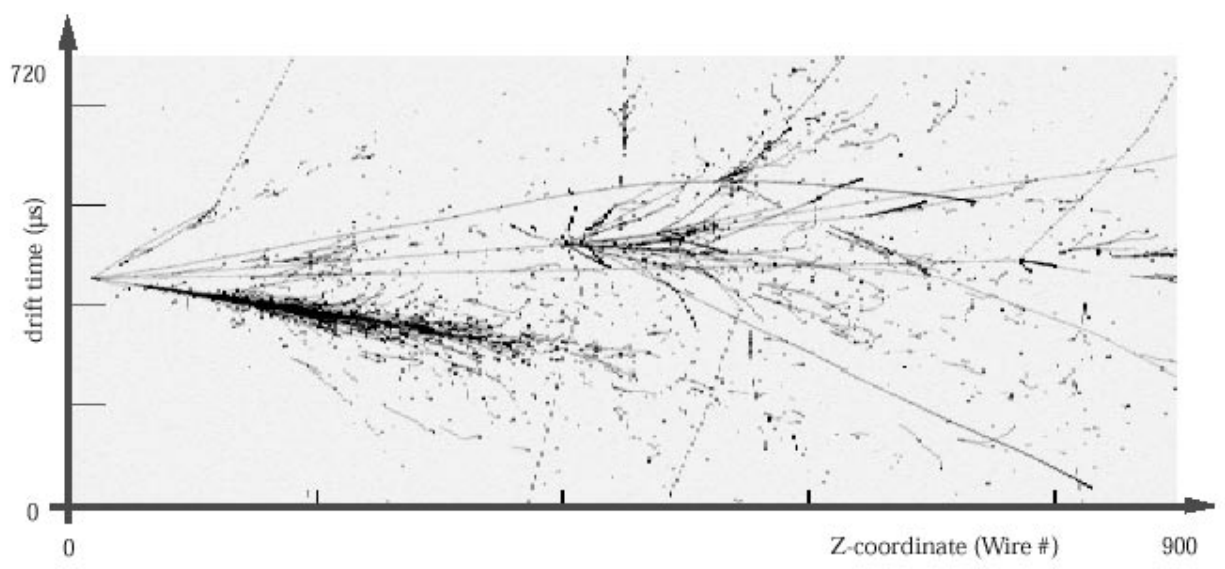

Figure 86: A simulated $\tau$ decay into electron in the ICARUS detector. The electron-induced electromagnetic shower is visible at the bottom part of the Figure.

operational at the Gran Sasso Laboratory. A simulated event obtained with this detector is shown in Fig. 86.

\subsubsection{The NOE calorimeter}

The NOE experiment $[45,227]$ was proposed for the CNGS beam. The search for $\nu_{\mu} \leftrightarrow \nu_{\tau}$ oscillations is performed with a large scale transition radiation detector (TRD) used for the detection of the electronic decay mode of the $\tau$. A search for $\nu_{\mu} \leftrightarrow \nu_{x}$ oscillations could also be accomplished by means of a massive calorimeter. The calorimeter is based on the scintillating fiber technique. It is subdivided into modules interleaved with TRD detector planes. A schematic view of the NOE detector is shown in Fig. 87.

A modified version of the NOE calorimeter is considered as solid target of the ICANOE supermodule. Its energy resolution for electrons and hadrons has been evaluated to be $\sigma(E) / E=20 \% / \sqrt{E(G e V)}+1 \%$ and $\sigma(E) / E=43 \% / \sqrt{E(G e V)}+1.5 \%$, respectively. The muon direction and the hadronic shower axis are measured with an angular resolution $\sigma_{\mu}(\theta)=1.27^{\circ} \sqrt{E_{\mu}}+2.27^{\circ} / E_{\mu}$ and $\sigma_{h}(\theta)=10^{\circ} / \sqrt{E_{h}}+20^{\circ} / E_{h}$, respectively.

\subsubsection{ICANOE performance}

Two classes of events can be used to search for neutrino oscillations with the ICANOE detector: interactions in the liquid and in the solid target. The latter event sample allows to perform a disappearance oscillation search thanks to the good muon momentum and hadronic energy measurements. For a restricted range of $\Delta m^{2}$ values one can also look for neutrino oscillations through the distortion of the predicted $\nu_{\mu} \mathrm{CC}$ spectrum. An event display of a simulated neutrino interaction detected by the liquid and solid detectors is shown in Fig. 88.

Due the excellent imaging capabilities of the liquid argon, the most sensitive search for neutrino oscilla- 


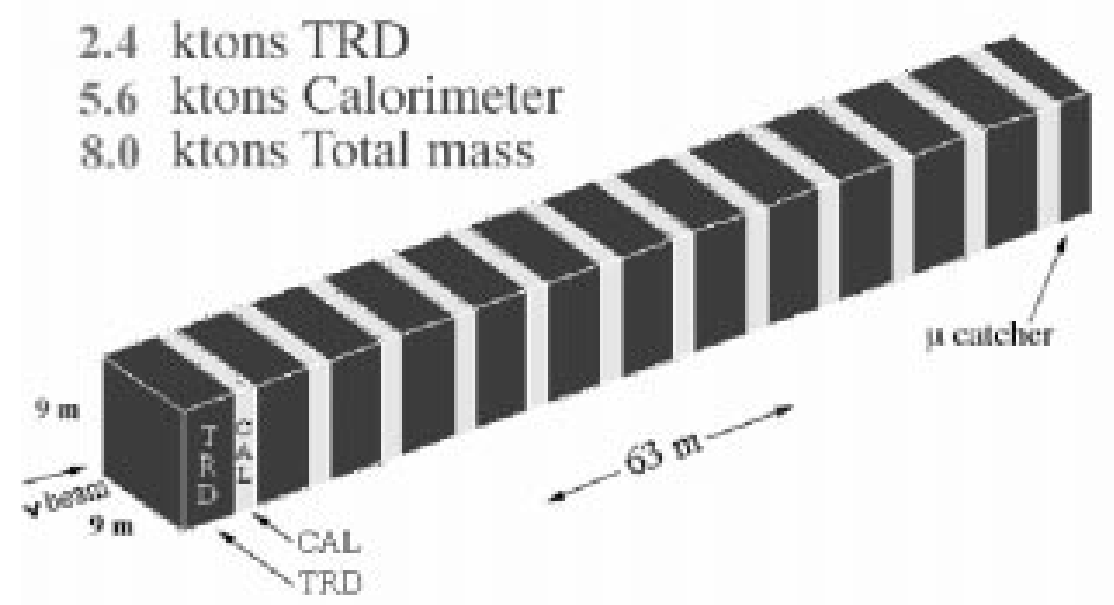

Figure 87: Isometric view of the original NOE detector.
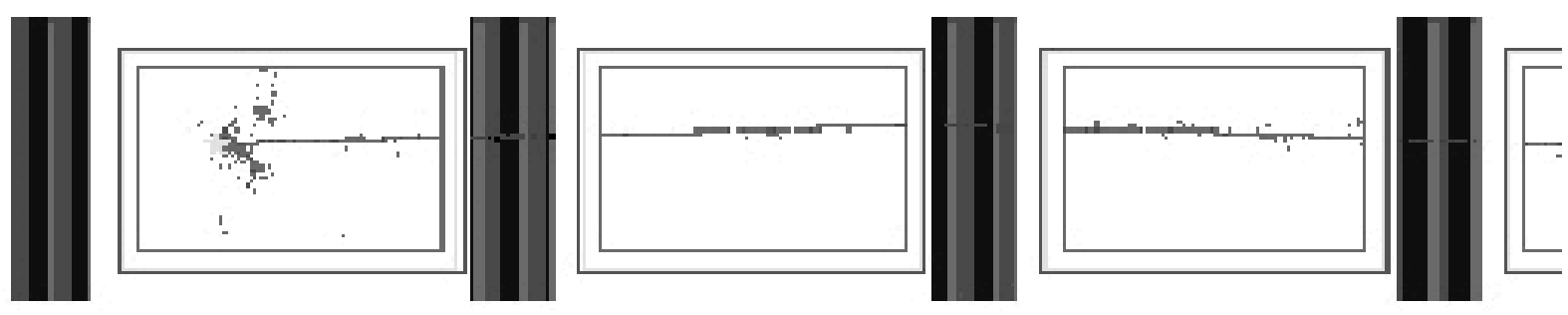

Figure 88: Simulated CNGS neutrino event in the ICANOE detector. 
tions is performed by using interactions occurring in the liquid target. A search for $\nu_{\mu} \leftrightarrow \nu_{\tau}$ oscillation can be accomplished by exploiting the different kinematics between $\nu_{\tau}$ and $\nu_{\mu}$ (or $\nu_{e}$ ) induced events.

The electron decay mode is the golden channel due to the high efficiency in detecting electromagnetic showers and the good resolution in determining their energy. The main selection criteria applied for the $\tau \rightarrow e$ analysis are given in Table 26 together with the expected number of background (from $\nu_{e}$ and $\bar{\nu}_{e}$ ) and signal events for different $\Delta m^{2}$ values. Four years of shared CNGS running are assumed.

Fig. 89 shows the total visible energy and the electron transverse momentum distributions for signal and background events. Details on how the $\nu_{\mu} \mathrm{CC}$ and $\nu_{\mu} \mathrm{NC}$ background can be rejected are given in [51].
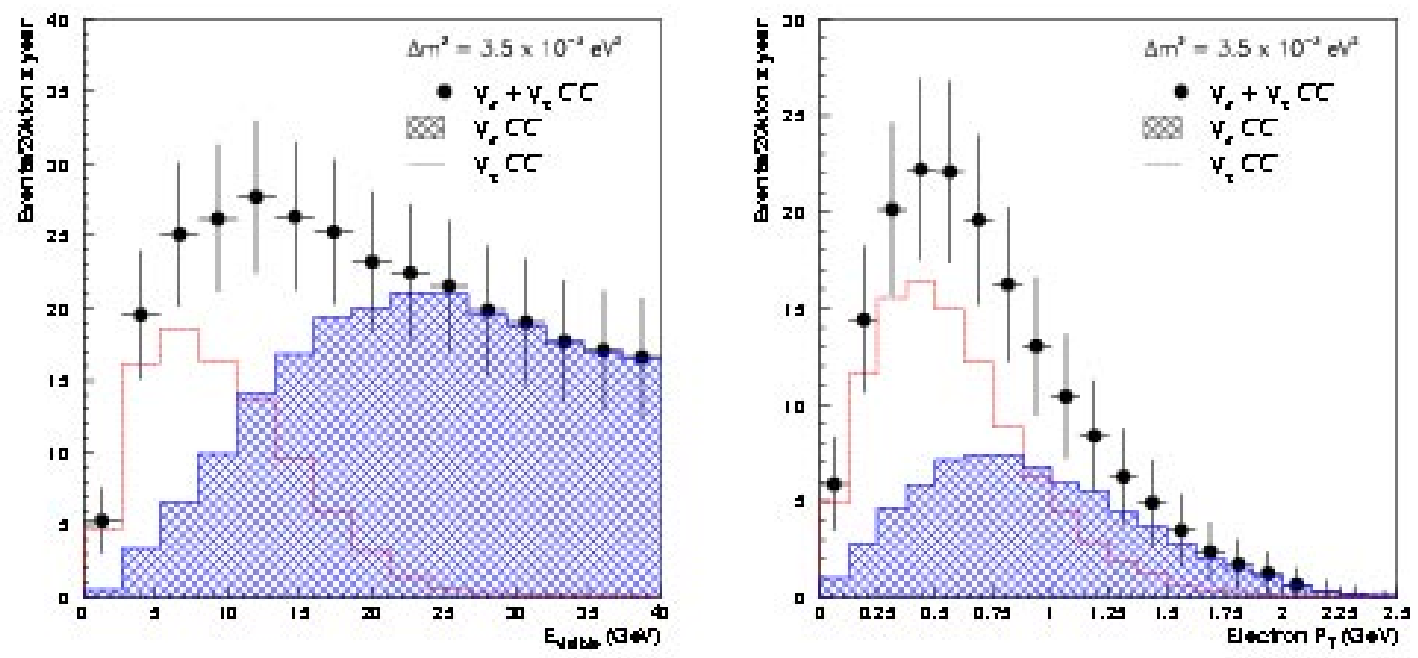

Figure 89: Signal and background in ICANOE. Left Figure: visible energy distribution. Right Figure: electron transverse momentum distribution. The statistics corresponds to 20 kton-year.

Once kinematics criteria and other cuts have been applied the expected number of signal events in the $\tau \rightarrow e$ channel amounts to 37 for the central parameter value indicated by Super-Kamiokande (Table 26). The estimated background is about 5 events. The sensitivity which can be achieved is shown in Fig. 84 .

Given the low $\nu_{e}$ and $\bar{\nu}_{e}$ contamination of the CNGS beam a search for $\nu_{\mu} \leftrightarrow \nu_{e}$ oscillations can also be performed. Assuming an overall $10 \%$ systematic error on the beam composition and exploiting a simple event counting together with a selection based on the visible energy of the event, the experiment will be able to probe the LSND claim. The corresponding 90\% C.L. exclusion plot is shown in Fig. 84.

\subsection{OPERA in the CNGS beam}

The OPERA experiment $[47-49,52,53]$ is meant for a long baseline search for $\nu_{\mu} \leftrightarrow \nu_{\tau}$ oscillations in the CNGS beam [109]. The experiment uses nuclear emulsions as high resolution tracking devices for the direct detection of the $\tau$ produced in the $\nu_{\tau}$ CC interaction with the target. Thanks to its capability of identifying electrons and to the small contamination of $\nu_{e}$ in the beam the experiment will also perform a $\nu_{\mu} \leftrightarrow \nu_{e}$ 


\begin{tabular}{||c|c|c|c|c|c|c||}
\hline \hline Cut & $\nu_{\tau}$ eff. & $\nu_{e} \mathrm{CC}$ & $\bar{\nu}_{e} \mathrm{CC}$ & $\begin{array}{c}\nu_{\tau} \text { events } \\
\left(\Delta \mathrm{m}^{2}=10^{-3} \mathrm{eV}^{2}\right)\end{array}$ & $\begin{array}{c}\nu_{\tau} \text { events } \\
\left(3.5 \times 10^{-3} \mathrm{eV}^{2}\right)\end{array}$ & $\begin{array}{c}\nu_{\tau} \text { events } \\
\left(10^{-2} \mathrm{eV}^{2}\right)\end{array}$ \\
\hline \hline Initial & 1 & 437 & 29 & 9.3 & 111 & 779 \\
\hline Fiducial volume & 0.88 & 383 & 25 & 8.2 & 97 & 686 \\
\hline $\begin{array}{c}\text { One candidate } \\
\text { with } p>1 \mathrm{GeV}\end{array}$ & 0.72 & 365 & 25 & 6.7 & 80 & 561 \\
\hline$E_{v i s}<18 \mathrm{GeV}$ & 0.67 & 64 & 5 & 6.2 & 75 & 522 \\
\hline$p_{T}^{e}<0.9 \mathrm{GeV}$ & 0.54 & 31 & 3 & 5.0 & 60 & 421 \\
\hline$p_{T}^{\text {lep }}>0.3 \mathrm{GeV}$ & 0.51 & 29 & 2 & 4.7 & 56 & 397 \\
\hline$p_{T}^{\text {miss }}>0.6 \mathrm{GeV}$ & 0.33 & 4 & 0.4 & 3.1 & 37 & 257 \\
\hline \hline
\end{tabular}

Table 26: Summary of the electron channel analysis for ICANOE.

oscillation search.

In the previous Sections we have already described three experiments (E531, CHORUS and TOSCA) based on the use of nuclear emulsions for $\nu_{\mu} \leftrightarrow \nu_{\tau}$ oscillation searches. This technique has found a large scale application in the CHORUS target [35], where the automatic scanning of a large sample of events has first been applied. In OPERA emulsions are used on an even larger scale but exclusively as high precision trackers unlike in CHORUS where they constitute the active target itself.

The feasibility of OPERA on the one hand requires the production of emulsions on an industrial scale and, on the other hand, it is linked to the impressive progress in the field of computer controlled microscopes read out by CCD cameras, with automatic pattern recognition and track reconstruction (Section 7.5). This progress has determined a real revolution in the field of nuclear emulsion detectors. They can nowadays be considered tracking devices and no longer visual detectors. The emulsions are like data storage devices whose data can be read out by automatic scanning systems without human intervention.

OPERA is designed starting from the Emulsion Cloud Chamber (ECC) concept (see references quoted in [52]) which combines the high precision tracking capabilities of nuclear emulsions and the large mass achievable by employing metal plates as a target. The ECC is a detector made of a sandwich of plates of passive material interspaced with nuclear emulsion layers used as high precision tracking devices. The ECC detection technique combined with the availability of automatic emulsion scanning devices allows to conceive and realize a $\sim 1000$ ton fine-grained vertex detector for the identification of $\nu_{\tau}$ appearance.

The sandwich structure is made up of cells. Two basic cell arrangements are envisaged for the identification of the $\tau$ decay kink through its direct detection. The first one, called compact cell, is shown in Fig. 90. The cell can be made of a $1 \mathrm{~mm}$ thick lead plate followed by a thin emulsion film. The film is made up of a pair of emulsion layers $50 \mu \mathrm{m}$ thick on either side of a $200 \mu \mathrm{m}$ plastic base. Charged particles give two track segments in each emulsion film. The number of grain hits in $50 \mu m(15-20)$ ensures redundancy in the measurement of particle trajectories.

If a $\tau$ is produced by a $\nu_{\tau}$ interacting in a lead plate it will decay either in the same plate (short decays) or further downstream (long decays). For long decays the $\tau$ is directly observed by measuring the angle between the charged decay daughter and the $\tau$ direction. This kink angle is caused by the invisible neutrino(s) produced in the decay (Section 7.5). For its measurement the directions of the tracks before and after the kink are reconstructed in space by means of a pair of emulsion films sandwiching the lead plate where the primary vertex occurred (Fig. 90). For short decays only an impact parameter measurement can 
be performed.

The electron and muon decay modes are characterised by low background and are considered as the main channels for the oscillation search. The electron channel, in particular, benefits from the dense lead-emulsion sandwich structure which allows the electron identification through its showering in the downstream cells. A source of background to the hadronic $\tau$ decays in the lead plates is given by hadron re-interactions. One of the primary hadrons may re-interact in the lead giving products not seen by the emulsion, hence simulating the charged one-prong decay of the $\tau$. The latter decay mode, however, can be exploited for the oscillation search by applying suitable cuts on the transverse momentum of the daughter particle.

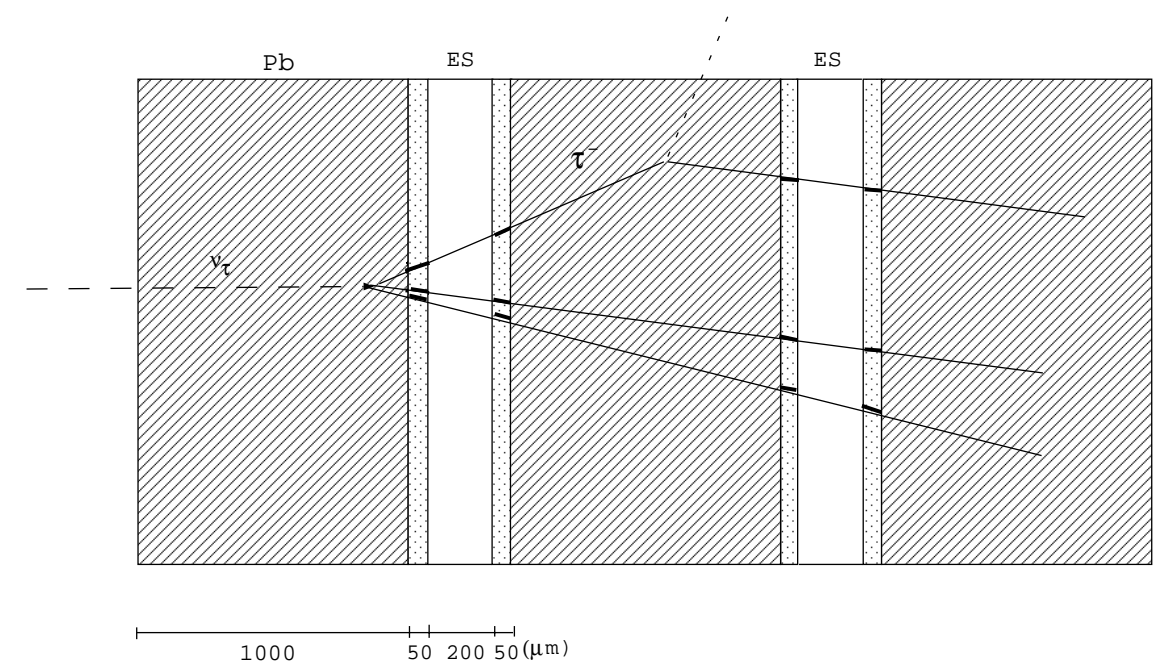

Figure 90: Schematic structure of an OPERA compact cell employing thin emulsion sheets. The $\tau$ decay kink is directly reconstructed in space by using four track segments in the emulsion films.

Another possible ECC cell structure (called spacer cell) foresees the insertion of a low density spacer between two emulsion sheets. This gap between the films allows direct detection of the $\tau$ decay kink as with the compact cell of Fig. 90. In this case the low density of the spacer makes the hadron re-interaction probability negligible. This results in a substantial background reduction for the $\tau$ hadronic decay channels which have the largest branching ratio. A possible design foresees a cell composed of a $1 \mathrm{~mm}$ thick lead plate followed by an emulsion film, a spacer of $3 \mathrm{~mm}$ and another film.

By piling up a series of cells in a sandwich-like structure one obtains the so-called brick which constitutes a detector element appropriate for the assembly in planar matrix structures (walls). Electronic trackers with moderate space resolution, placed downstream of each emulsion brick wall, are needed to identify the brick where the neutrino interaction took place and to guide the scanning, so providing time resolution to the emulsions. Their main task is to determine the event shower-axis, hence locating the brick whose emulsion must be scanned.

Once the fired brick has been identified is removed from the detector and its emulsions developed and scanned. Starting from the most downstream emulsion film in the brick, track segments are detected and measured in position and angle. This procedure continues in the other films until the interaction vertex plate is found. The search for a decay topology is performed with the emulsion film just downstream of the vertex lead plate. Fig. 91 shows the simulation of a $\nu_{\mu} \mathrm{CC}$ event occurring inside a brick. 
An important requirement for the experiment is the determination of the decay transverse momentum of the daughter particle. This is a tool to reject background events. For most of the muons this measurement can be accomplished by means of the muon spectrometers. For electrons the energy is estimated from the shower development. For charged hadrons the momentum is measured by multiple scattering in the ECC sandwich structure. We refer to [52] for a description of the above methods and of the results of test. In [52] one can also find a tentative design of the detector and an overview of the expected performance of the experiment. The proposal of OPERA with the definition of the detector baseline design is expected by July $2000[228]$.

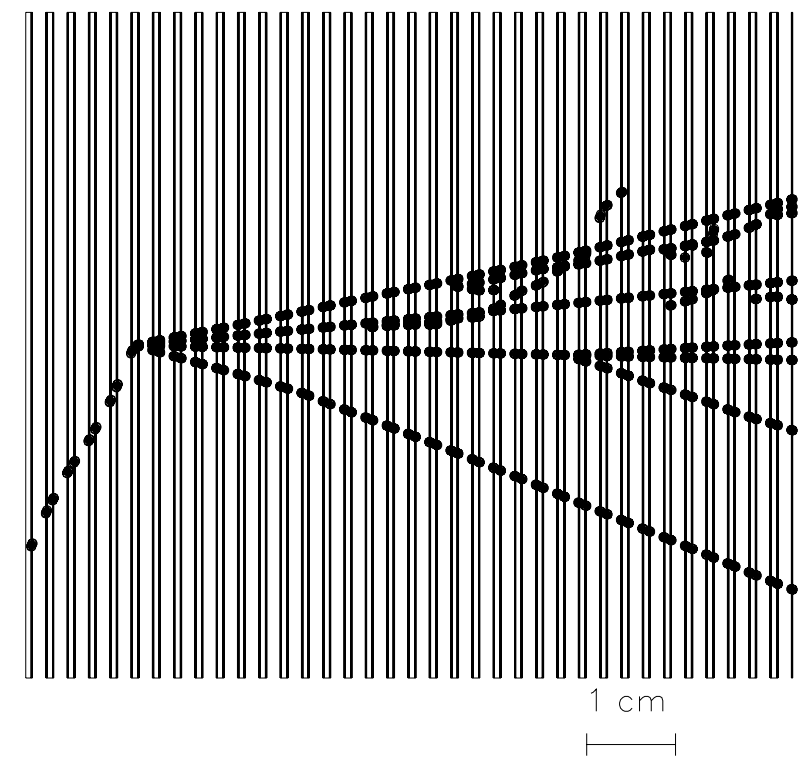

Figure 91: Simulated $\nu_{\mu}$ CC event in one brick of OPERA. The isolated primary muon track (from bottomright) points to the vertex.

\subsubsection{Detector strucure}

The detector design is based on a modular structure. A wall and its related electronic detector planes constitutes a module. A supermodule is defined by a target section made of several consecutive modules followed by a muon detection system. The structure of the target permits the removal of the fired bricks and to analyse their emulsion sheets during the run.

The dimensions of the bricks are determined by conflicting requirements. A small brick size favours the optimal use of the emulsions when considering brick removals after interactions. The integrated mass to be taken out has to be small compared to the total target mass. In addition, bricks have to be small and light enough to allow easy handling by automatic machines. On the other hand, losses due to edge effects are reduced with larger brick cross-section. Another issue is the brick thickness in units of radiation lengths. It determines the particle identification capabilities when following tracks in consecutive cells and also the 
momentum measurement by multiple scattering. In the tentative design of [52] each brick has dimensions of about $15 \times 15 \mathrm{~cm}^{2}$ orthogonal to the beam direction. Compact bricks consist of 56 cells with a total thickness of about $7 \mathrm{~cm}\left(10 X_{0}\right)$ and a weight of about $14.5 \mathrm{~kg}$.

Module and supermodule dimensions are governed by the efficient use of the space in the underground Gran Sasso cavern. This would motivate large transverse dimensions of the modules. However, one has to be confronted with the requirements of large surface electronic detectors, with the realistic dimensions of the muon spectrometers, with the mechanical design and with the need for sufficient space for services and for the handling of bricks. These considerations favour a transverse wall dimensions of about $7 \mathrm{~m}$. The shape of the brick wall could be rectangular or octagonal in order to match the actual shape of the supermodule muon spectrometer. The number of modules in a supermodule, and hence its longitudinal dimension, is basically determined by the muon acceptance and thus by the maximum realistic size of the spectrometers. This limits the longitudinal length of a target supermodule to $\sim 3 \mathrm{~m}$.

A muon spectrometer constitutes the downstream part of each supermodule. Its purpose is to identify muons with high efficiency in order to reduce the charm background and to measure the sign of the charge. The number of single and associated charm background events produced in $\nu_{\mu} \mathrm{CC}$ interactions is proportional to the primary muon identification inefficiency. The design goal is an overall muon identification efficiency of $95 \%$.

The background given by charmed mesons produced in $\nu_{\mu} \mathrm{CC}$ and $\mathrm{NC}$ interactions and decaying into muons is further reduced by the measurement of the muon charge. The reduction factor is proportional to the charge determination efficiency of the decay muon. Each spectrometer is made of three high resolution trackers interleaved with two toroidal or dipolar magnets made of steel plates. The spectrometers have to provide the required momentum resolution of at least $30 \%$ in the relevant kinematical domain.

\subsubsection{Detection efficiency and background}

The first quantity contributing to the detection efficiency is the brick finding efficiency determined by the electronic trackers. Sources of inefficiencies are, in the longitudinal direction, back-scattering at the event vertex and, in the transverse direction, finite resolution effects at the boundary between adjacent bricks as well as interactions in the material of the support frames. A global brick detection efficiency $\sim 90 \%$ is estimated [52].

Another source of inefficiency is represented by fiducial volume cuts. Tracks can be reconstructed up to very close to the film edge $(\sim 1 \mathrm{~mm})$. The effect of the dead space for the brick-to-brick event connection leads to an additional inefficiency. Considering the various contributions the result is an overall geometrical efficiency of about $80 \%$.

The relatively low background of tracks stored in the emulsions facilitates the procedure of vertex finding. One can estimate the vertex finding efficiency $\varepsilon_{v e r t}$ either by Monte Carlo simulations or by simulations using real data, e.g. from CHORUS. It amounts to $\sim 80 \%$ on average for all $\tau$ decay channels.

Some of the factors which contribute to the detection efficiency are independent of the $\tau$ decay channel. A $\tau$ produced in $\nu_{\tau}$ CC interactions and decaying outside the vertex plate is identified through a direct kink detection. Given the CNGS beam features and the $\tau$ kinematics the probability for the $\tau$ to exit the vertex plate is $45 \%$ for $\Delta m^{2}=3.5 \times 10^{-3} \mathrm{eV}^{2}$.

An additional factor entering into the detection efficiency is given by the rejection of small-angle and 
very large-angle kinks. The upper cut $(>500 \mathrm{mrad}$ ) is presently motivated by reasons of scanning efficiency of the automatic microscopes. The lower cut is due to the angular resolution in the measurement of the kink angle in the space. Test beam measurements indicate that the angular resolution per projection measured by means of four track segments in the emulsion film is $\sigma_{\theta} \sim 2 \mathrm{mrad}$, while it becomes $\sigma_{\theta} \sim 10 \mathrm{mrad}$ for decays in the base [52]. This motivates a lower cut of about $25 \mathrm{mrad}$. The fraction of $\tau$ decays with a kink angle in the useful range depends of the actual decay mode. It ranges from 85 to $90 \%$.

In particular for compact bricks, contributions to the detection efficiency come from the electron identification efficiency and from kinematical cuts needed to reject hadronic re-interactions or muon scattering in the lead plate mimicking a $\tau$ decay.

The overall signal detection efficiency for long decay events for compact supermodules amounts to $\sim 6 \%$ by considering the muon and the electron $\tau$ decay modes. The possibility of using also one-prong hadronic decays and short decays is presently under study [228].

The main background sources to long $\tau$ decays are: cosmic rays and radioactivity, hadronic decays and re-interactions, muon scattering and one-prong decay of charmed particles.

The kink finding procedure is based on the space association of track segments reconstructed with the two emulsion films of a cell. The segments corresponding to the $\tau$ and to its daughter are searched for over an emulsion surface which depends on the distance between the two consecutive films. Therefore, one has to consider the potential background given by accidental association with isolated track segments which could originate from beam-unrelated sources. The main beam unrelated sources are given by $\beta$ rays from lead radioactivity and cosmic-ray tracks accumulated before the installation at Gran Sasso. Both backgrounds are expected to be kept at an acceptable level by suitable selection of the lead and by exploiting the so-called controlled fading of the emulsions [52].

Muon scattering in the lead could be a source of background for the muonic decay of the $\tau$. In this case a muon may undergo a scattering in the lead plate following the vertex plate hence mimicking a muonic $\tau$ decay.

Charmed particles are produced in $\mathrm{CC}$ and $\mathrm{NC}$ neutrino interactions through the reactions

$$
\text { a) } \nu_{\mu} N \rightarrow c \mu X, \quad \text { b) } \nu_{\mu} N \rightarrow c \bar{c} \mu X, \quad \text { c) } \nu_{\mu} N \rightarrow c \bar{c} \nu_{\mu} X
$$

Charmed mesons have mass and lifetime similar to those of the $\tau$. The above processes may thus constitute a background to the oscillation signal if one fails to detect the primary muon (a), the charm partner (c) or both (b). The most relevant source is given by single charm production (reaction (a)).

\subsubsection{Sensitivity to $\nu_{\mu} \leftrightarrow \nu_{\tau}$ oscillations}

Table 27 summarises the number of signal and background events which can be collected in the various channels by one supermodule made of spacer or compact bricks with $2.25 \times 10^{20}$ pot from the SPS. This corresponds to a nominal five years' run of the CNGS.

In order to cover the Super-Kamiokande allowed region [15] five OPERA supermodules are required according to the design outlined in [52]. One could then obtain $\sim 20 \tau$ events if $\nu_{\mu} \leftrightarrow \nu_{\tau}$ oscillations occur at the Super-Kamiokande central value of $\Delta m^{2}=3.5 \times 10^{-3} \mathrm{eV}^{2}$ and full mixing, with less than of one background event. 


\begin{tabular}{||c|c|c|c|c|c||}
\hline \hline Supermodule & $\tau$ decay mode & $\begin{array}{c}\text { Signal } \\
\left(2 \times 10^{-3} \mathrm{eV}^{2}\right)\end{array}$ & $\begin{array}{c}\text { Signal } \\
\left(3.5 \times 10^{-3} \mathrm{eV}^{2}\right)\end{array}$ & $\begin{array}{c}\text { Signal } \\
\left(6 \times 10^{-3} \mathrm{eV}^{2}\right)\end{array}$ & BG \\
\hline spacer & $e^{-}$ & 0.26 & 0.81 & 2.38 & 0.010 \\
\hline " & $\mu^{-}$ & 0.23 & 0.70 & 2.06 & 0.004 \\
\hline " & $h^{-}$ & 0.64 & 1.97 & 5.79 & 0.036 \\
\hline compact & $e^{-}$ & 0.65 & 2.00 & 5.88 & 0.040 \\
\hline & $\mu^{-}$ & 0.56 & 1.73 & 5.08 & 0.16 \\
\hline \hline
\end{tabular}

Table 27: $\tau$ and background events detected by one spacer or compact OPERA supermodule [52]. Full mixing and three values of $\Delta m^{2}$ are assumed. The values are given for $2.25 \times 10^{20}$ pot .

The low expected background of OPERA is a key feature to achieve the physics goal. The sensitivity to $\Delta m^{2}$ improves with the square root of the number of detected events. This implies that already after a run of about two years a significant limit could be set in the case of a negative search. This is shown in Fig. 92, where the exclusion plots at the $90 \%$ C.L. are given for a two, three or four years' run. The Figure also shows the result from a global fit of the different Super-Kamiokande measurements on atmospheric neutrinos.

In the case of a positive result, values for the oscillation parameters could be inferred. This is shown in Fig. 93. An experimental systematic error of $10 \%$ has been assumed on the estimated numbers of signal and background events. The accuracy in the determination of the $\Delta m^{2}$ parameter is similar to that of the ICANOE experiment.

\section{Future experiments with beams from muon storage rings}

\subsection{Neutrino beams from muon decay}

Neutrino beams can be made from the decay of muons accumulated in storage rings. This purely leptonic process allows to get rid of the uncertainties related to hadronic reactions which are relevant in the case of neutrino beams from pion decays. Therefore, fluxes can be easily predicted. Moreover, one has the freedom of choosing the neutrino spectrum by fixing the storage ring energy.

Given the decay chains

$$
\begin{aligned}
& \mu^{-} \rightarrow e^{-} \nu_{\mu} \bar{\nu}_{e} \\
& \mu^{+} \rightarrow e^{+} \nu_{e} \bar{\nu}_{\mu}
\end{aligned}
$$

an equal number of $\nu_{e}\left(\bar{\nu}_{e}\right)$ and $\bar{\nu}_{\mu}\left(\nu_{\mu}\right)$ populate the beam contrary to the case of beams from pion decay (Section 6.1). 


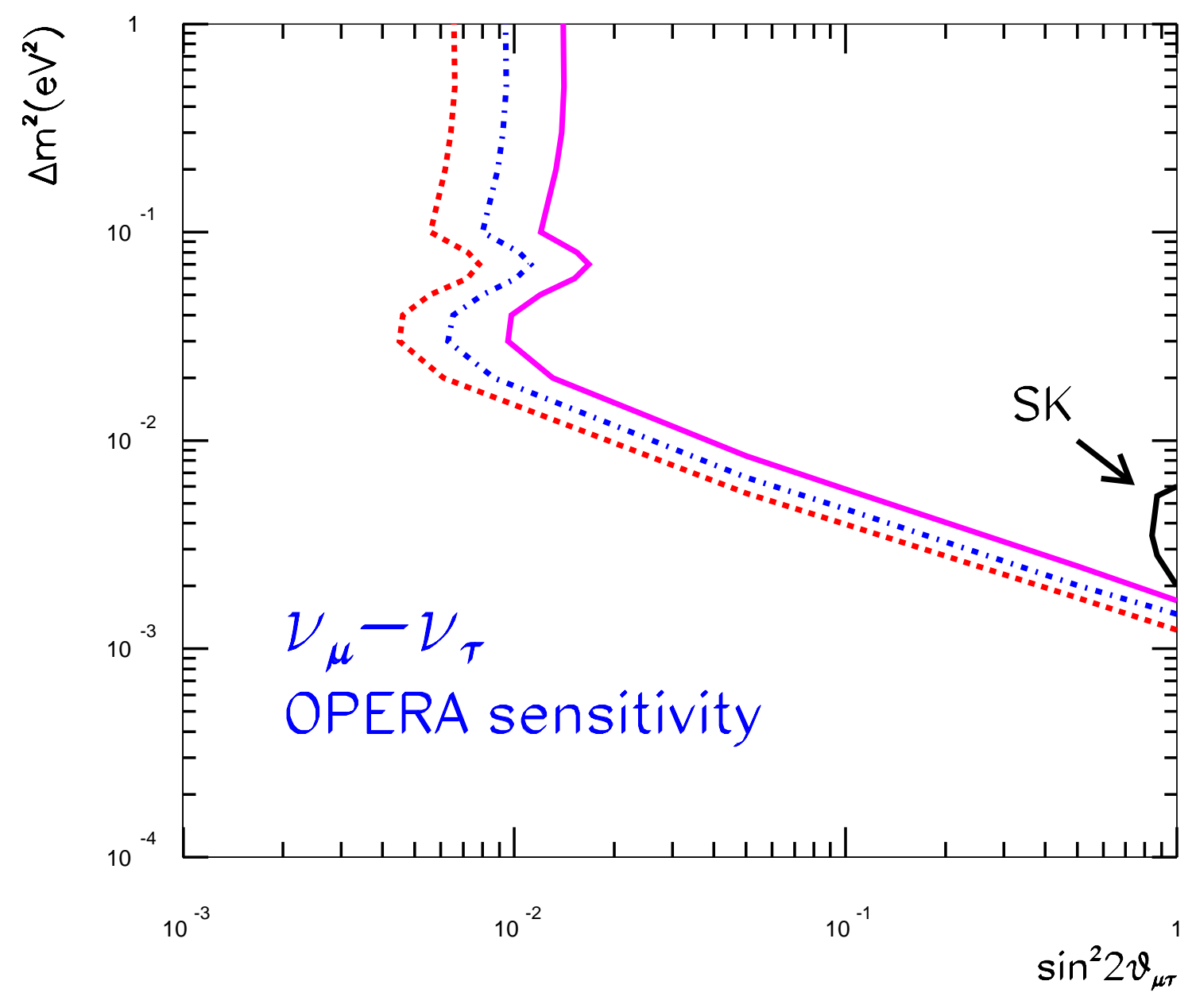

Figure 92: Sensitivity of OPERA in the search for $\nu_{\mu} \leftrightarrow \nu_{\tau}$ oscillations. The $90 \%$ C.L. exclusion limits (2, 3 and 4 years' run) are plotted together with the region allowed by Super-Kamiokande. 


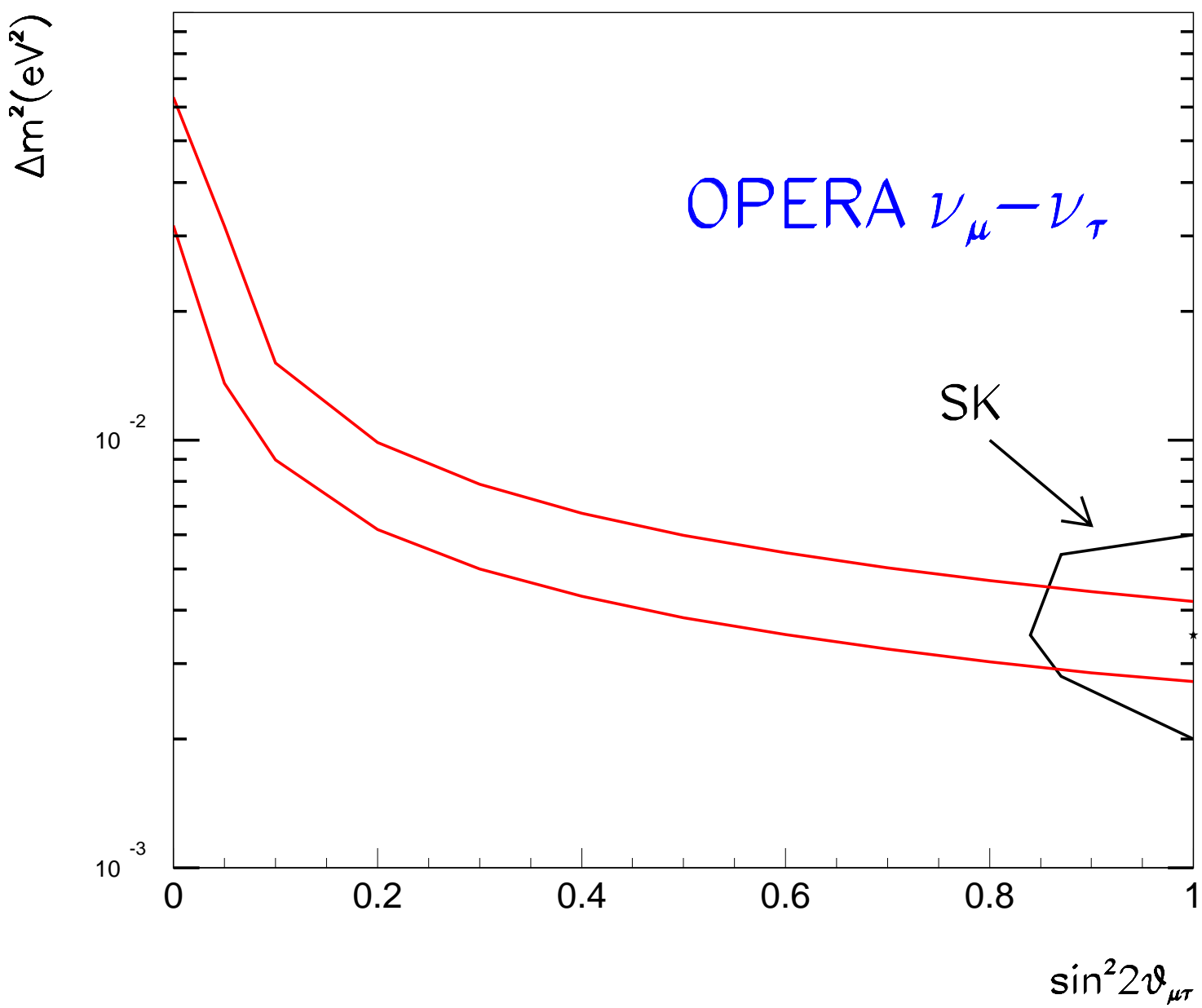

Figure 93: OPERA allowed parameter region (90\% C.L.) obtained under the assumption that $\nu_{\mu} \leftrightarrow \nu_{\tau}$ oscillations occur at the preferred Super-Kamiokande values. 
A description of the general features of such beams can be found in $[54,57]$. Muons are allowed to decay in a straight section of a storage ring. The neutrino energy and angular distributions are functions of the parent muon energy, of the decay angle and of the muon spin direction. The construction of a muon storage ring facility could be the first step towards the design of high energy muon colliders [229].

The neutrino beam intensity can be orders of magnitude higher than that of conventional beams. It depends on the rate at which muons are stored in the ring and hence on the muon source. The source can consist of a proton accelerator, a target for the production of pions, a focusing system, a decay channel and a muon cooling section.

It can be shown [54] that a suitable design could allow for the production of about $2 \times 10^{20}$ neutrinos (or anti-neutrinos) per year. The expected CC rates for the different neutrino flavours are shown in Fig. 94 as a function of the muon energy. Under the assumption of $20 \mathrm{GeV}$ unpolarised muons, of absence of neutrino oscillations and of a site $10000 \mathrm{~km}$ away from the source (e.g. as for a beam from Fermilab to Super-Kamiokande) one would expect of the order of $2.2 \times 10^{20} \bar{\nu}_{\mu} \mathrm{m}^{-2}$ year $^{-1}$ and the same number of $\nu_{e}$.

Preliminary studies on neutrino sources from muon storage rings have been performed and the possible use of the existing accelerator facilities (CERN, Fermilab) was considered [230-232]. Further studies will be needed with the aim of defining a realistic design.

\subsection{LBL experiments with muon storage rings}

The main motivation of LBL experiments with high intensity neutrino beams from muon storage rings is the precision study of neutrino mixing, whose existence can be inferred from the signal indicated by atmospheric neutrino experiments. A first clarification of the scenario is expected with the forthcoming generation of LBL experiments with beams from pion decays as outlined in the previous Sections.

LBL $(\sim 1000 \mathrm{~km})$ or very long baseline experiments $(\sim 10000 \mathrm{~km})$ with muon decay beams will consolidate the knowledge of neutrino mixing and will also allow to start a complete programme of neutrino oscillation physics in a general three-flavour mixing scheme. Additional physics subjects could be the detection of Earth matter effects and possible $\mathrm{CP}$ and $\mathrm{T}$ violation in the leptonic sector $[55,233,234]$. This will be accomplished by means of the intense neutrino and anti-neutrino beams which could be obtained by changing the polarity of the stored muons.

All the relevant oscillation channels could be searched for. The $\nu_{\mu} \leftrightarrow \nu_{\tau}$ or $\nu_{\mu} \leftrightarrow \nu_{\text {sterile }}$ oscillation parameters will be precisely measured once K2K, NuMI and CNGS experiments have confirmed the SuperKamiokande signal. A possible admixture of the $\nu_{\mu} \leftrightarrow \nu_{\tau}$ and $\nu_{\mu} \leftrightarrow \nu_{\text {sterile }}$ channels could also be detected.

Very long baseline experiments can be designed taking into account the possible locations of accelerator complexes and underground laboratories [54-57,235]. This is schematically shown in Fig. 95 [56]. In particular, one can conceive to send a beam from the Fermilab to the Gran Sasso Laboratory with a $7332 \mathrm{~km}$ baseline. It can be shown [57] that with a $20 \mathrm{GeV}$ muon storage ring and with a 1 kton OPERA-like detector one could collect from $\sim 15$ to $45 \tau$ events per year if $\nu_{\mu} \leftrightarrow \nu_{\tau}$ oscillations occur within the parameter region presently indicated by Super-Kamiokande [15]. Analogously, with a 10 kton-year massive detector a $\nu_{\mu}$ disappearance experiment would determine the oscillation probability at a few percent level.

A $\tau$ appearance experiment with $730 \mathrm{~km}$ baseline (such as from Fermilab to the Soudan mine or from CERN to Gran Sasso) could also be considered. This is schematically depicted in Fig. 96 where the single- 


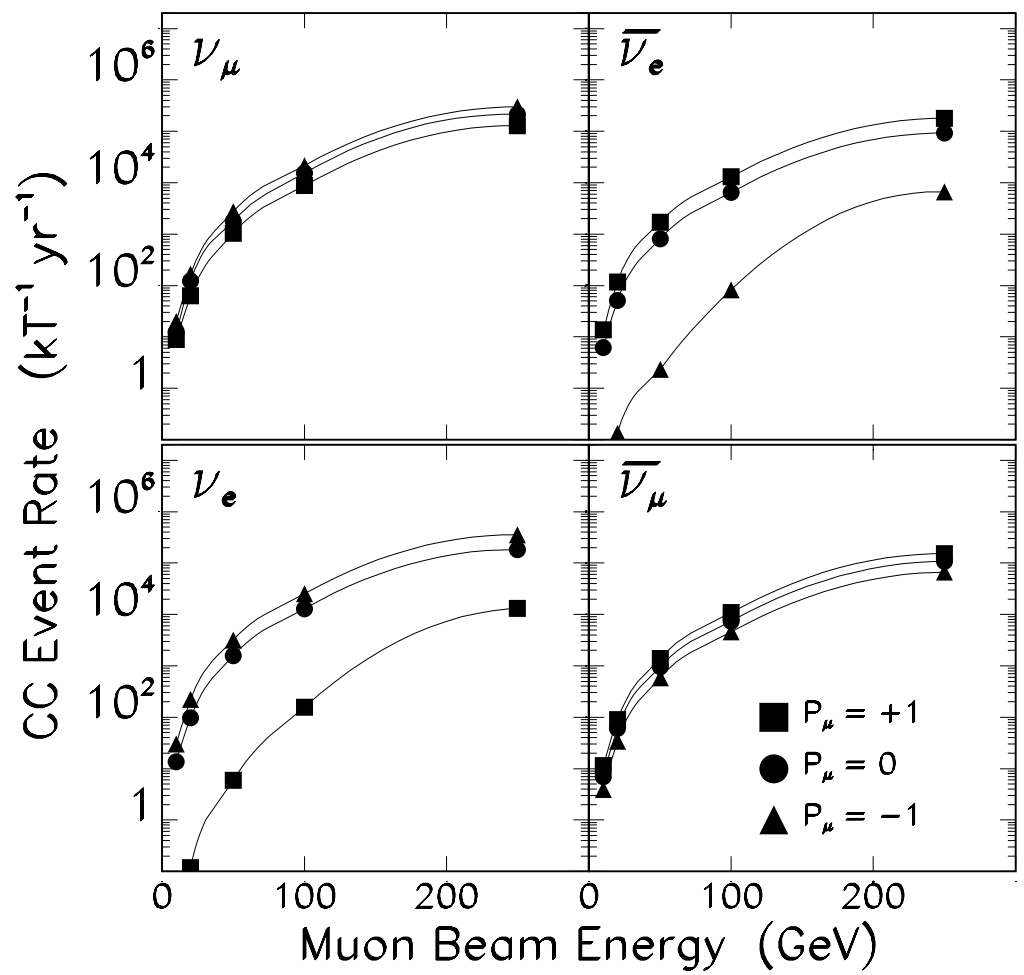

Figure 94: Expected CC event rates at a location $10000 \mathrm{~km}$ distant from a muon storage ring source. Three possible muon polarisations are contemplated. 


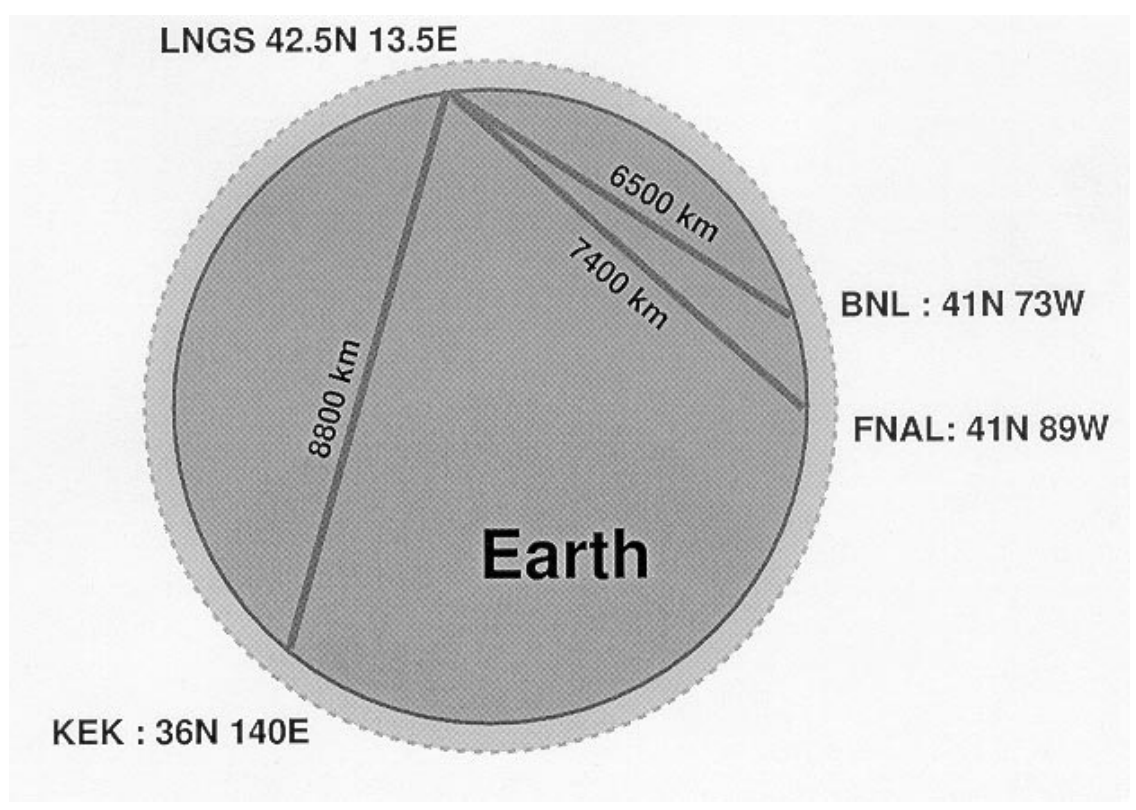

Figure 95: Possible very long baseline beams across the Earth (seen from the North pole).

event sensitivity contours of such a $\nu_{\mu} \leftrightarrow \nu_{\tau}$ experiment are shown for different muon energies [57]. In that case, however, with a realistic detector one would not have anymore a background-free experiment, as in the case of the $\sim 7000 \mathrm{~km}$ baseline. The $730 \mathrm{~km}$ baseline would be preferred if $\nu_{\mu} \leftrightarrow \nu_{\text {sterile }}$ oscillations dominated. One could then perform a high sensitivity disappearance experiment also capable to detect Earth matter effects [57].

In a similar way, a beam from a muon storage ring could contribute to the study of the LSND signal, following the possible confirmation by the next generation experiments (e.g. MiniBooNE). $\nu_{e} \leftrightarrow \nu_{\tau}$ and $\nu_{e} \leftrightarrow \nu_{\mu}$ could be searched for with high sensitivity. This is shown in Fig. 97 where the single-event per year contour limits are shown as a function of the muon energy for a $10 \mathrm{kton}$ detector at $730 \mathrm{~km}$ distance. The LMA solution of the solar neutrino deficit could be probed as well by such an experiment.

\section{Conclusions}

We have attemped to review the main experiments searching for neutrino oscillations with particle accelerator beams. A brief introduction to the theory and phenomenology of neutrino oscillations has been given and the different experimental methods employed for the detection of neutrino oscillations have been outlined.

After a long series of negative results there are presently a few positive experimental indications for neutrino oscillations. The strongest comes from the observed deficit of $\nu_{\mu}$ produced in the atmosphere by cosmic rays measured by the Super-Kamiokande experiment. In the usual two-flavour mixing scheme a small $\Delta m^{2}\left(\sim 10^{-2}-10^{-3} e V^{2}\right)$ and a large mixing angle $\left(\sin ^{2} 2 \theta>0.8\right)$ are derived. No evidence exists for $\nu_{\mu} \leftrightarrow \nu_{e}$ oscillations in the same parameter domain, favouring the hypothesis of $\nu_{\mu} \leftrightarrow \nu_{\tau}$ or, to a lesser 


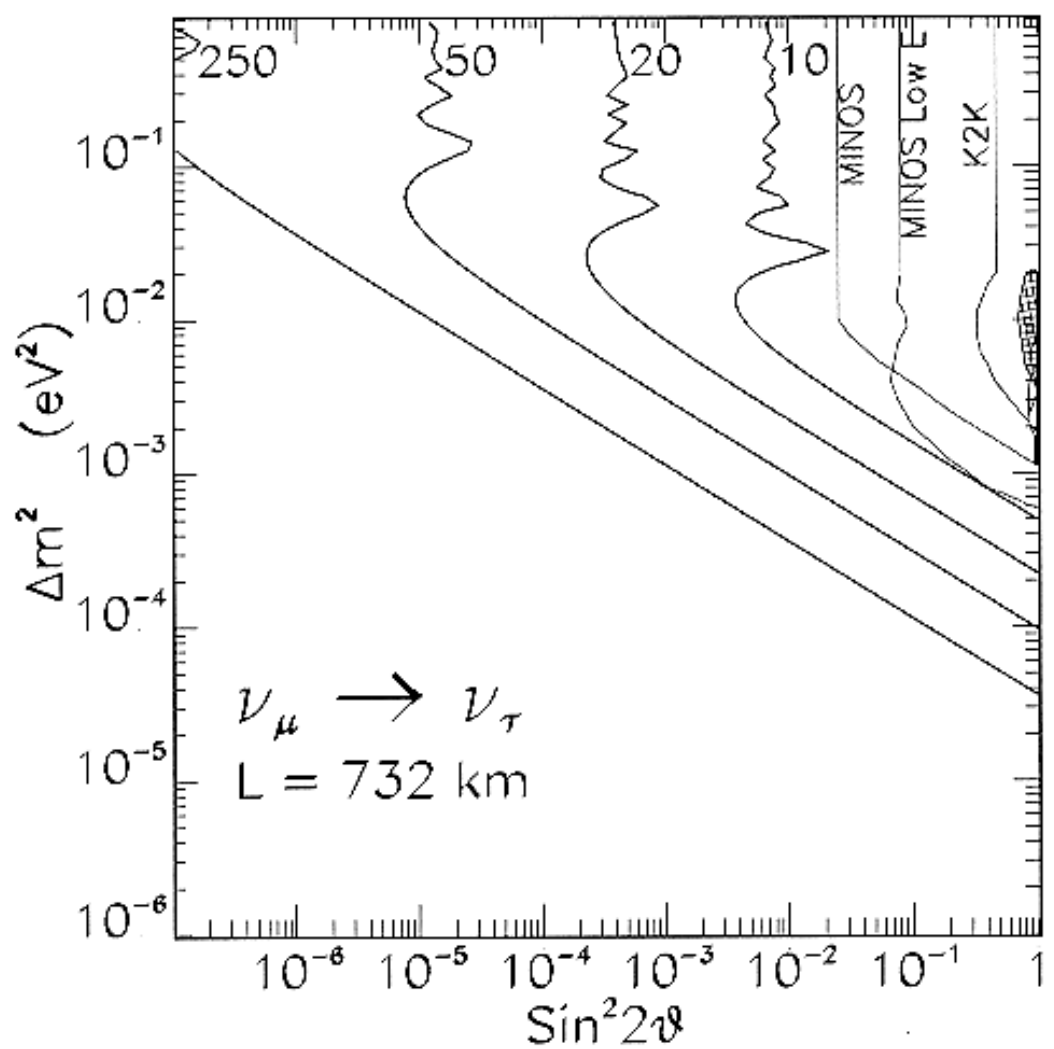

Figure 96: $\nu_{\mu} \leftrightarrow \nu_{\tau}$ : single-event per year contours. $10 \mathrm{kton}$ detector at $732 \mathrm{~km}$ distance from a storage ring neutrino source. Different muon energies are contemplated. 


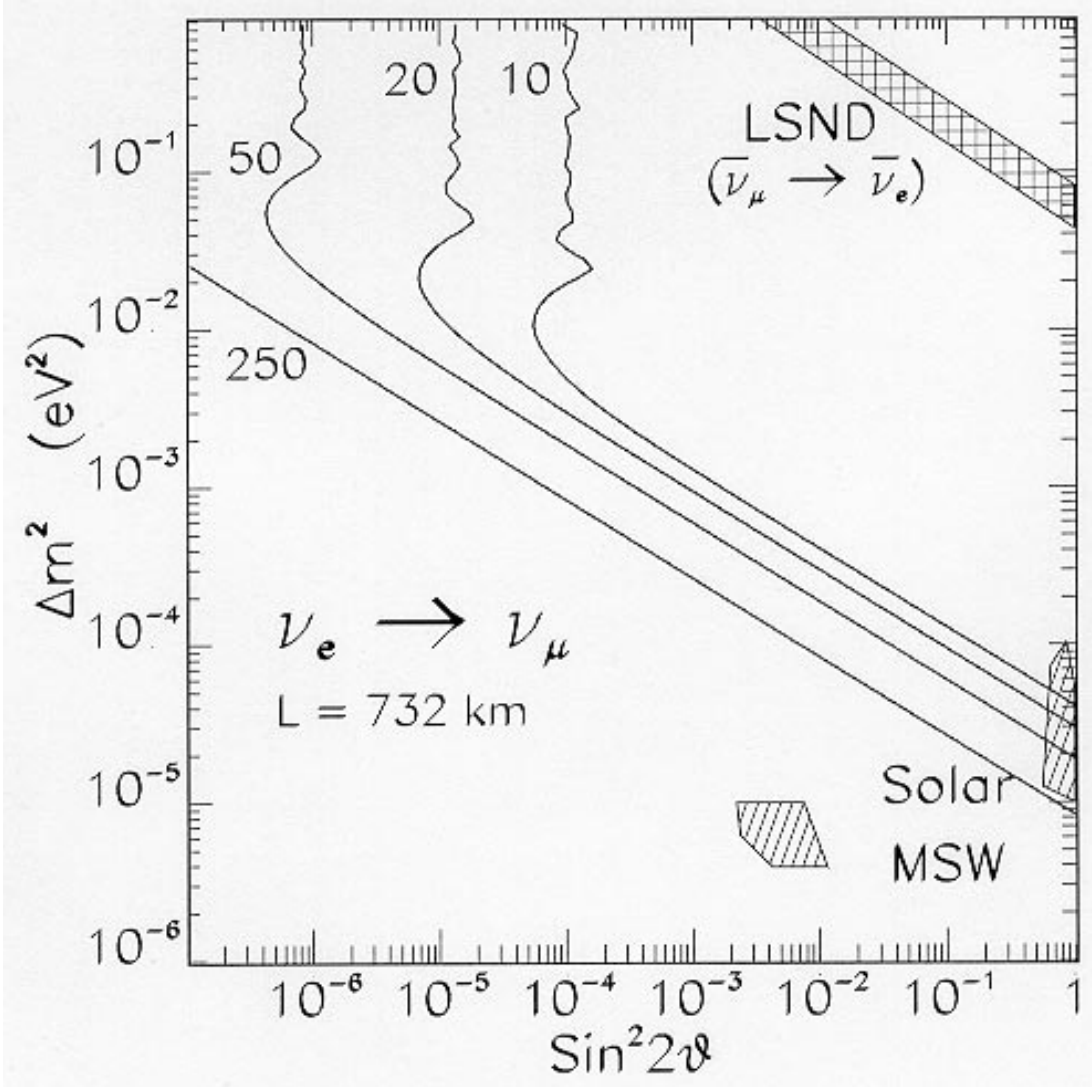

Figure 97: $\nu_{e} \leftrightarrow \nu_{\mu}$ : single-event per year contours. $10 \mathrm{kton}$ detector at $732 \mathrm{~km}$ distance from a storage ring neutrino source. Different muon energies are contemplated. 
extent, $\nu_{\mu} \leftrightarrow \nu_{\text {sterile }}$ oscillations.

The detected solar $\nu_{e}$ flux which is about a factor two smaller than predicted by solar models can also be explained in terms of neutrino oscillations, of which it provided the first evidence. In addition, the claim from the LSND accelerator experiment suggests the existence of $\bar{\nu}_{\mu} \leftrightarrow \bar{\nu}_{e}$ oscillations with $\Delta m^{2} \sim 1 e V^{2}$.

The next generation of solar neutrino experiments is expected to substantially contribute to the experimental scenario of neutrino mixing. With atmospheric neutrinos, the Super-Kamiokande experiment will continue to take data aiming at a better determination of the mixing parameters and at improving in the

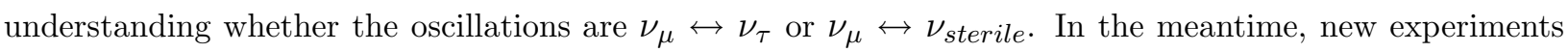
can be conceived to detect the expected $L / E$ modulation of the atmospheric neutrino signal and possibly improve the Super-Kamiokande results.

Experiments with neutrinos from accelerators are being conducted and others are planned or proposed. These experiments could test a large domain of the oscillation parameters including the LSND region, the possibility of a cosmologically relevant neutrino mass and the atmospheric neutrino signal.

Long baseline experiments will probe the $\Delta m^{2}$ region indicated by atmospheric neutrinos. The interest of the community on this subject is reflected in the number of proposed long baseline experiments with $\nu_{\mu}$ beams produced by accelerators.

The first long baseline neutrino beam was commissioned in 1999 from KEK to the Super-Kamiokande detector about $250 \mathrm{~km}$ away. At Fermilab the experiment MINOS will use the NuMI beam from Fermilab to the Soudan mine at $730 \mathrm{~km}$ distance to test the hypothesis of neutrino oscillations with $\Delta \mathrm{m}^{2} \sim 10^{-2}-$ $10^{-3} \mathrm{eV}^{2}$. Finally, the ICANOE and OPERA experiments have been proposed to exploit the recently approved CNGS beam from CERN to the Gran Sasso Laboratory, also in this case about $730 \mathrm{~km}$ away from the source.

In five-ten years from now we hope that the forthcoming generation of experiments will have established the existence of neutrino masses and mixing. This will then motivate further precision experiments aimed at the study of the neutrino mass matrix and, possibly, at the understanding of the mechanism of fermion mass generation. High intensity neutrino beams from muon storage rings could be realised for this purpose and used together with a new generation of neutrino detectors. By then, the neutrino will certainly have revealed some of its mysteries and, maybe, have also led to intriguing new puzzles...

\section{Acknowledgements}

The authors wish to warmly acknowledge the kind help of many colleagues in providing information and material for this review. In particular, we are indebted to R. Bernstein, J. Cobb, J.M. Conrad, L. Di Lella, M. Goodman, J. Hylen, B. Kayser, J. Kleinfeller, B. Louis, A. Marchionni, Y. Oyama, F. Pietropaolo, A. Rubbia, K. Scholberg and P. Zucchelli. We would also like to express our gratitude to U. Dore, J. Panman, B. Saitta and P. Strolin for their critical reading of the paper and the relevant suggestions. Finally, we are pleased to thank S. Anthony for the careful reading of the manuscript and R. Peluso for the help with graphic and editing tools.

\section{References}

[1] Particle Data Group, Euro. Phys. J. 1 (1998) 1. 
[2] J.R. Primack and M.A.K. Gross, Proc. of the X Rencontres de Blois: The Birth of Galaxies, Blois, 1998 (astro-ph/9810204) and references therein.

[3] Ch. Weinheimer et al., Phys. Lett. B 460 (1999) 219.

[4] V.M. Lobashev et al., Phys. Lett. B 460 (1999) 227.

[5] K. Assamagan et al., Phys. Rev. D 53 (1996) 6065.

[6] R. Barate et al., Europ. Phys. J. C 2 (1998) 395.

[7] B. Pontecorvo, Zh. Eksp. Teor. Fiz. 33 (1957) 549.

[8] B. Pontecorvo, Zh. Eksp. Teor. Fiz. 34 (1958) 247.

[9] Z. Maki et al., Prog. Theor. Phys. 28 (1962) 870.

[10] V. Gribov and B. Pontecorvo, Phys. Lett. B 28 (1969) 493.

[11] J.N. Bahcall et al., Phys. Lett. B 433 (1998) 1.

[12] S.P. Mikheyev and A.Yu. Smirnov, Yad. Fiz. 42 (1985) 1441.

[13] L. Wolfenstein, Phys. Rev. D 17 (1985) 2369.

[14] J.N. Bahcall et al., Phys. Rev. D 58 (1998) 096016.

[15] http://www-sk.icrr.u-tokio.ac.jp/doc/sk/index.html;

Y. Fukuda et al., Phys. Rev. Lett. 81 (1998) 1562;

Y. Fukuda et al., Phys. Rev. Lett. 82 (1999) 5194;

M. Nakahata, Proc. of the VI Int. Workshop on Topics in Astroparticle and Underground Physics, Paris, 1999.

[16] http://duphy4.physics.drexel.edu/chooz-pub/

[17] M. Apollonio et al., Phys. Lett. B 420 (1998) 397.

[18] M. Apollonio et al., Phys. Lett. B 466 (1999) 415.

[19] C. Athanassopoulos et al., Phys. Rev. Lett. 81 (1998) 1774.

[20] C. Athanassopoulos et al., Phys. Rev. C 58 (1998) 2489.

[21] C. Athanassopoulos et al., Phys. Rev. C 54 (1996) 2685.

[22] C. Athanassopoulos et al., Phys. Rev. Lett. 77 (1996) 3082.

[23] http://www.sno.phy.queensu.ca/

[24] G. Ewan et al., Sudbury Neutrino Observatory Proposal, SNO-87-12 (1987).

[25] http://almime.mi.infn.it/

[26] C. Arpesella et al., Borexino Proposal, Vols. 1 and 2, ed. G. Bellini and R. S. Raghavan, Univ. of Milan, (1991).

[27] F.v. Feilitzsch et al, Progr. Part. Nucl. Phys. 40 (1998) 123.

[28] G. Alimonti et al., Astro. Phys. 8 (1998) 141.

[29] http://www.awa.tohoku.ac.jp/html/KamLAND/index.html

[30] A. Suzuki, Proc. of the VIII Int. Workshop on Neutrino Telescopes, Venice, 1999.

[31] M. Ambrosio et al., LNGS-LOI 20/99, CERN/SPSC 99-24 SPSC/M636.

[32] T. Jannakos, Proc. of the XXXIV Rencontres de Moriond: Electroweak Interactions and Unified Theories, Les Arcs, 1999 (hep-ex/9908043).

[33] http://www.neutrino.lanl.gov/BooNE/

[34] M. Guler et al., CERN-SPSC/99-26, SPSC/P311.

[35] M. de Jong et al., CERN-PPE/93-131.

[36] P. Astier et al., CERN-SPSLC/91-21 (1991), CERN SPSLC/9148, SPSLC/P261 Add.1.

[37] CHORUS Coll., Contrib. to the XIX Int. Symposium on Lepton and Photon Interactions at High Energies (LP 99), Stanford, 1999 (hep-ex/9907015).

[38] P. Astier et al., Phys. Lett. B 453 (1999) 169.

[39] http://neutrino.kek.jp/

[40] K. Nishikawa et al., KEK Report E362 (1995).

[41] Y. Oyama, Proc. of the YITP Workshop on Flavor Physics, Kyoto, 1998 (hep-ex/9803014).

[42] H.W. Sobel, Proc. of the VIII Int. Workshop on Neutrino Telescopes, Venice, 1999. 
[43] E. Ables et al., Fermilab Proposal P-875 (1995);

NuMI-L-337 (1998);

NuMI-L-375 (1998);

http://www.hep.anl.gov/NDK/Hypertext/numi.html

[44] ICARUS Proposal, LCNGS-94/99-I (1994);

J.P. Revol et al., ICARUS-TM-97/01;

ICARUS Coll. CERN/SPSC-98-33.

[45] M. Ambrosio et al., Nucl. Instr. and Meth. A 363 (1995) 604;

CERN/SPSC-98-32.

[46] P. Antonioli et al., CERN/SPSC-98-37.

[47] A. Ereditato et al., INFN/AE-97/06 and Nagoya DPNU-97-07, 27 January 1997.

[48] H. Shibuya et al., CERN/SPSC-97-24, LNGS-LOI 8/97.

[49] K. Kodama et al., CERN/SPSC-98-25;

CERN/SPSC-99-20, SPSC/M635, LNGS-LOI 19/99.

[50] M. Apollonio et al., CERN/SPSC-98-34.

[51] F. Arneodo et al., INFN/AE-99-17, CERN/SPSC-99-25, SPSC/P314.

[52] K. Kodama et al., CERN/SPSC-99-20, SPSC/M635, LNGS-LOI 19/99.

[53] http://www.cern.ch/opera/

[54] S. Geer, Phys. Rev. D 57 (1998) 6989.

[55] A. De Rujula et al., Nucl. Phys. B 547 (1999) 21.

[56] M. Campanelli et al., hep-ex/9905240, 1999.

[57] V. Barger et al., Phys. Rev. D 61 (2000) 053004.

[58] S. M. Bilenky, hep-ph/9908335, 1999.

[59] M. Nakagawa, Proc. of the Europhysics Neutrino Oscillation Workshop (NOW 98), Amsterdam, 1998 (hep-ph/9811358).

[60] S.M. Bilenky and B. Pontecorvo, Phys. Rep. C 41 (1978) 225.

[61] V. Flaminio and B. Saitta, Riv. Nuovo Cim. 10 (1987) 1.

[62] S.M. Bilenky and S.T. Petcov, Rev. Mod. Phys. 59 (1987) 671.

[63] B. Kayser, The physics of massive neutrinos, World Scientific, Singapore, 1989.

[64] Y. Takeuchi et al., hep-ph/9809558, 1998.

[65] S.M. Bilenky et al., Prog. Part. Nucl. Phys. 43 (1999) 1.

[66] P. Langacker, Testing the Standard Model, World Scientific, Singapore, 1991.

[67] G. Gelmini and E. Roulet, Rep. Progr. Phys. 58 (1995) 1207

[68] S.M. Bilenky, Europ. School of High Energy Physics, Casta Papiernicka, 1999 (hep-ph/0001311).

[69] E.Kh. Akhmedov, hep-ex/0001264, 2000.

[70] B. Kayser, Phys. Rev. D 24 (1981) 110.

[71] M. Zralek, lecture given at the XXXVIII Cracow School of Theoretical Physics, Zakopane, 1998 (hep$\mathrm{ph} / 9810543)$.

[72] S. Nussinov, Phys. Lett. B 63 (1976) 201.

[73] J.M. Conrad, Proc. of the XXIX Int. Conf. on High Energy Physics (ICHEP), Vancouver, 1998 (hepex/9811009).

[74] J. Cobb, Nucl. Instr. and Meth. A 372 (1996) 501.

[75] P.F. Loverre, Phys. Lett. B 206 (1988) 711.

[76] G.J. Feldman and R.D. Cousins, Phys. Rev. D 57 (1998) 3873.

[77] R. Davis Jr. et al., Phys. Rev. Lett. 20 (1968) 1205.

[78] J.N. Bahcall et al., Phys. Rev. Lett. 20 (1968) 1209.

[79] B.T. Cleveland et al., Astrophys. J. 496 (1998) 505.

[80] W. Hampel et al., Phys. Lett. B 447 (1999) 127.

[81] J. N. Adburashitov et al., Phys. Rev. C 60 (1999) 055801.

[82] W. Hampel et al., Phys. Lett. B 420 (1998) 114. 
[83] J.N. Abdurashitov et al., Phys. Rev. C 59 (1999) 2246.

[84] W. Hampel et al., Phys. Lett. B 436 (1998) 158.

[85] Y. Fukuda et al., Phys. Rev. Lett. 77 (1996) 1683.

[86] Y. Suzuki, Proc. of the XIX Int. Symposium on Lepton and Photon Interactions at High Energies, Stanford University, 1999.

[87] M. Nakahata et al., Nucl. Instr. and Meth. A 421 (1999) 113.

[88] V. Barger et al., Phys. Rev. D 22 (1980) 2718.

[89] T.K. Kuo and J. Pantaleone, Phys. Rev. D 35 (1987) 3432.

[90] M. Honda et al., Phys. Lett. D 52 (1995) 4985.

[91] T.K. Gaisser et al., Phys. Lett. D 54 (1996) 5578.

[92] M. Aglietta et al., Europhys. Lett. 8 (1989) 611.

[93] K. Daum et al., Z. Phys. C 66 (1995) 417.

[94] D. Casper et al., Phys. Rev. Lett. 66 (1991) 2561.

[95] R. Becker-Szendy et al., Phys. Rev. D 46 (1992) 3720.

[96] T. Kafka, Proc. of the VI Int. Workshop on Topics in Astroparticle and Underground Physics, Paris, 1999, (hep-ex/9912060).

[97] Y. Fukuda et al., Phys. Lett. B 335 (1994) 237.

[98] Y. Fukuda et al., Phys. Rev. Lett. 82 (1999) 2644.

[99] F. Ronga, Proc. of the VI Int. Workshop on Topics in Astroparticle and Underground Physics, Paris, 1999 ;

M. Ambrosio et al., hep-ex/0001044, 2000.

[100] B. Pontecorvo, J. Exptl. Theoret. Phys. 37 (1959) 1751.

[101] M. Schwartz, Phys. Rev. Lett. 4 (1960) 306.

[102] G. Danby et al., Phys. Rev. Lett. 9 (1962) 36.

[103] M.M. Block et al., Phys. Lett. 12 (1964) 281.

[104] K. Borer et al., Phys. Lett. B 29 (1969) 614.

[105] E. Heijne, CERN Yellow Report 83-06, 1983.

[106] G. Acquistapace et al., CERN Preprint, CERN-ECP/95-14, 1995.

[107] E. Eskut et al., Nucl. Instr. and Meth. A 401 (1998) 7.

[108] G.N. Taylor et al., Phys. Rev. D 28 (1983) 2705.

[109] G. Acquistapace et al., CERN 98-02, INFN/AE-98/05, 1998.

[110] A. Fassò et al., Proc. of the III Workshop on Simulating Accelerator Radiation Environment, SARE-3, KEK-Tsukuba, H. Hirayama ed., KEK report Proc. 97-5, p. 32 (1997).

[111] A. Ferrari et al., in Proc. of the III Workshop on Simulating Accelerator Radiation Environment, SARE-3, KEK-Tsukuba, H. Hirayama ed., KEK report Proc. 97-5, p. 165 (1997).

[112] A. Ereditato et al., ICARUS-TM-98/13, OPERA 980722-01.

[113] G. Battistoni and P. Lipari, Proc. of the Vulcano Workshop on Frontier Objects in Astrophysics and Particle Physics, 1998 (hep-ph/9807475).

[114] http://www.cern.ch/NGS

[115] R. Bailey et al., CERN-SL/99-034(DI), INFN/AE-99/05, 1999.

[116] http://www.nikhef.nl/discuss/ (Now98 - Beams).

[117] The Fermilab NuMI Group, Fermilab Report NuMI-346, 1998.

[118] GEANT 3.21, CERN Program Library Long Writeup W5013.

[119] H. Noumi et al., Nucl. Instr. and Meth. A 398 (1997) 399.

[120] C. Baltay, Proc. Int. Conf. on Neutrino Physics and Astrophysics, Haway, 1981.

[121] H. Wachsmuth, Proc. Int. Conf. on Neutrino Physics and Astrophysics, Balatonfüred, 1982.

[122] M.H. Shaevitz, Int. Lepton and Photon Symposium, Cornell, 1983.

[123] F.W. Bullock and R.C.E. Devenish, Rep. Prog. Phys. 46 (1983) 1029.

[124] C. Grupen, Particle detectors, Cambridge University Press and references therein.

[125] E. Bellotti et al., Lett. Nuovo Cim. 17 (1976) 553. 
[126] J. Blietschau et al., Nucl. Phys. B 133 (1978) 205.

[127] H. Deden et al., Phys. Lett. B 98 (1981) 310.

[128] O. Erriquez et al., Phys. Lett. B 102 (1981) 73.

[129] N. Armenise et al., Phys. Lett. B 100 (1981) 182.

[130] A.M. Cooper et al., Phys. Lett. B 112 (1982) 97.

[131] C. Angelini et al., Phys. Lett. B 179 (1986) 307.

[132] F. Dydak et al., Phys. Lett. 134 (1984) 281.

[133] F. Bergsma et al., Z. Phys. C 40 (1988) 171.

[134] K. de Winter et al., Nucl. Instr. and Meth. A 278 (1989) 670.

[135] D. Geiregat. et al., Nucl. Instr. and Meth. A 325 (1993) 92.

[136] P. Vilain et al., Phys. Lett. B 335 (1994) 246.

[137] P. Vilain et al., Phys. Lett.B 345 (1995) 115.

[138] M. Gruwé et al., Phys. Lett. B 309 (1993) 463.

[139] http://choruswww.cern.ch/

[140] H. Harari, Phys. Lett. B 216 (1989) 413.

[141] Ya.B. Zel'dovich and I.D. Novikov, Relativistic Astrophysics, Nakua, Moscow, 1967.

[142] J. Ellis et al., Phys. Lett. B 292 (1992) 189.

[143] H. Fritzsch and D. Holtmannspötter, Phys. Lett. B 338 (1994) 290.

[144] S. Aoki et al., Nucl. Instr. and Meth. B 51 (1990) 446.

[145] T. Nakano, Ph.D. Thesis, University of Nagoya (1997).

[146] V. Tioukov, Proc. of the I Int. Workshop on Nuclear Emulsion Techniques, Nagoya, 1998.

[147] P. Pelosi, Proc. of the I Int. Workshop on Nuclear Emulsion Techniques, Nagoya, 1998.

[148] J. Panman, Proc. of the I Int. Workshop on Nuclear Emulsion Techniques, Nagoya, 1998.

[149] E. Eskut et al., Phys. Lett. B 424 (1998) 202.

[150] E. Eskut et al., Phys. Lett. B 434 (1998) 205.

[151] N. Ushida et al., Phys. Lett. B 206 (1988) 375.

[152] P. Annis et al., Phys. Lett. B 435 (1998) 458.

[153] K. Kodama et al., FERMILAB P803 Proposal (1993), Appendix D.

[154] F. Baldassarre et al., Nuovo Cimento XXI, 3 (1961) 459.

[155] B. Van der Vyver, Nucl. Instr. and Meth. A 385 (1997) 91.

[156] R.D. Cousins and V.L. Highland, Nucl. Instr. and Meth. A 320 (1992) 331.

[157] http://nomadinfo.cern.ch/

[158] J. Altegoer et al., Nucl. Instr. and Meth. A 404 (1998) 96.

[159] J. Altegoer et al., Phys. Lett. B 431 (1998) 219.

[160] P.F. Salvatore, Proc. of the XXXIV Rencontres de Moriond: Electroweak Interactions and Unified Theories, Les Arcs, 1999.

[161] V.V. Ammosov et al., Z. Phys. C 40 (1988) 487.

[162] L.A. Ahrens et al. Phys. Rev. D 31 (1985) 2732.

[163] P. Astier et al., Nucl. Phys. B 335 (1990) 517.

[164] G. Bernardi et al., Phys. Lett. B 181 (1986) 173.

[165] L.A. Ahrens et al. Phys. Rev. Lett. 51 (1983) 1514.

[166] L.A. Ahrens et al. Phys. Rev. Lett. 54 (1985) 18.

[167] B. Blumenfeld et al., Phys. Rev. Lett. 62 (1989) 2237.

[168] L. Borodovsky et al., Phys. Rev. Lett. 68 (1992) 274.

[169] A.M. Cnops et al., Phys. Rev. Lett. 40 (1978) 144.

[170] N.J. Baker et al., Phys. Rev. Lett. 47 (1981) 1576.

[171] A.E. Asratyan et al., Phys. Lett. B 105 (1981) 301.

[172] Yu. Batusov et al., Z. Phys. C 48 (1990) 209. 
[173] E.B. Brucker et al., Phys. Rev. D 34 (1986) 2183.

[174] J. Bofill et al., Phys. Rev. D 36 (1987) 3309.

[175] B.J. King et al., Nucl. Instr. and Meth. A 302 (1991) 254.

[176] K.S. McFarland et al., Phys. Rev. Lett. 75 (1995) 3993.

[177] A. Romosan et al., Phys. Rev. Lett. 78 (1997) 2912.

[178] N. Ushida et al., Phys. Rev. Lett. 57 (1986) 2897.

[179] K. Niwa, Physics and Astrophysics of Neutrinos, editors M. Fukugita and A. Suzuki, Springer-Verlag, (1994) 520.

[180] S.G. Frederiksen, University of Ottawa, Ph. D. Thesis (unpublished), 1987.

[181] http://www.neutrino.lanl.gov/LSND/

[182] P. Némethy et al., Phys. Rev. D 23 (1981) 262.

[183] L.S. Durkin et al., Phys. Rev. Lett. 61 (1988) 1811.

[184] S.J. Freedman et al., Phys. Rev. D 47 (1993) 811.

[185] J.A. McGill et al., Los Alamos National Laboratory Report LA-8937T, 1981.

[186] J.L. Langenbrunner et al., Nucl. Instr. and Meth. A 325 (1993) 187.

[187] R.L. Burman et al., Nucl. Instr. and Meth. A 368 (1996) 416.

[188] C. Athanassopoulos et al., Phys. Rev. C 56 (1997) 2806.

[189] C. Athanassopoulos et al., Nucl. Instr. and Meth. A 388 (1998) 149.

[190] D.H. White, Proc. of the XVIII Int. Conf. on Neutrino Physics and Astrophysics, Takayama, 1998.

[191] E. Kolbe et al., Phys. Rev. C 49 (1994) 1122.

[192] E. Kolbe et al., Phys. Rev. C 52 (1995) 3437.

[193] D. Rein and L.M. Sehgal, Nucl. Phys. B 223 (1983) 29.

[194] http://www-ik1.fzk.de/www/karmen/

[195] B. Zeitnitz et al., Prog. in Part. and Nucl. Phys. 32 (1994) 351.

[196] B. Zeitnitz et al., Prog. in Part. and Nucl. Phys. 40 (1998) 169.

[197] G. Drexlin et al., Prog. in Part. and Nucl. Phys. 32 (1994) 375.

[198] G.L. Fogli et al., Phys. Rev. D 49 (1994) 3626.

[199] G.L. Fogli et al., Phys. Rev. D 54 (1996) 2048.

[200] G.L. Fogli et al., Phys. Rev. D 56 (1997) 4365.

[201] P.F. Harrison et al., Phys. Lett. B 349 (1995) 137.

[202] A. Acker and S. Pakvasa, Phys. Lett. B 397 (1997) 209.

[203] C.Y. Cardall and G.M. Fuller, Phys. Rev. D 53 (1996) 4421.

[204] C.Y. Cardall et al., Phys. Lett. B 413 (1997) 246.

[205] B. Kayser, Proc. of the XXIX Int. Conf. on High Energy Physics (ICHEP), Vancouver, 1998 (hep$\mathrm{ph} / 9810513)$.

[206] M. Gonzalez-Garcia, Proc. of the XXIX Int. Conf. on High Energy Physics (ICHEP), Vancouver, 1998.

[207] S. Nandi, Proc. of the XXIX Int. Conf. on High Energy Physics (ICHEP), Vancouver, 1998.

[208] P. Roy, Proc. of the XXIX Int. Conf. on High Energy Physics (ICHEP), Vancouver, 1998.

[209] R.P. Thun and S. McKee, Phys. Lett. B 439 (1998) 123.

[210] G. Barenboim and F. Scheck, Phys.Lett. B 440 (1998) 332.

[211] T. Sakai and T. Teshima, Prog. Theor. Phys. 102 (1999) 629.

[212] T. Ohlsson and H. Snellman, Phys. Rev. D 60 (1999) 093007.

[213] B. Achkar et al., Nucl. Phys. B 434 (1995) 503.

[214] G.L. Fogli et al., Phys. Rev. D 59 (1999) 033001.

[215] G.L. Fogli et al., Phys. Rev. D 56 (1997) 3081.

[216] V. Barger et al., Phys. Rev. D 58 (1998) 93016.

[217] COSMOS Coll., Fermilab Proposal P803, (1993).

[218] A. Ereditato et al., Nucl. Phys. 54 B (1997) 139. 
[219] J.J. Gomez-Cadenas et al., Nucl. Instr. and Meth. A 378 (1996) 196;

J.J. Gomez-Cadenas and J.A. Hernando, Nucl. Instr. and Meth. A381 (1996) 223.

[220] A. Ayan et al., CERN-SPSC/97-5;

A. Ayan et al., CERN-SPSC/98-20.

[221] J.J. Gomez-Cadenas, Proc. of the VI VERTEX-96 Conference, Cagliari, 1996;

D. Ferrere, Proc. of the V Int. Conf. on Advanced Technology and Particle Physics, Como, 1996.

[222] D. Allasia et al., Nucl. Phys. B 176 (1980) 13.

[223] Y. Oyama, Proc. of the XXXV Rencontres de Moriond, Les Arcs, 2000 (hep-ex/0004015).

[224] http://pcnometh4.cern.ch/

[225] http://www.aquila.infn.it/icarus/

[226] F. Arneodo et al., Proc. of the INFN Eloisatron Project XXXVI Workshop: New Detectors, Erice, 1997 (hep-ex/9812006).

[227] http://www1.na.infn.it/wsubnucl/accel/noe/noe.html

[228] OPERA Coll., CERN/SPSC 2000-012, SPSC/M615, LNGS-LOI, Add.3.

[229] R. Palmer et al., Proc. of the 1996 DPF/DPB Summer Study on New Directions for High Energy Physics, Snowmass '96, Snowmass, 1996 (unpublished).

[230] B. Autin, Proc. of the Muon Collider Coll. Workshop, St. Croix, 1999.

[231] D. Finley et al., FERMILAB-TM-2072, 1999.

[232] A. Blondel et al., CERN Preprint, CERN-EP/2000-53, 2000.

[233] N. Cabibbo, Phys. Lett. B 72 (1978) 333.

[234] V. Barger et al., Phys. Rev. D 59 (1999) 113010.

[235] P. Migliozzi and P. Strolin, Proc. of the V ICFA/ECFA Workshop on Neutrino Factories Based on Muon Storage Rings (NuFact 99), Lyon, 1999. 